\title{
Unified Reasoning about Robustness Properties of Symbolic-Heap Separation Logic
}

\author{
Christina Jansen ${ }^{1}$, Jens Katelaan ${ }^{2}$, Christoph Matheja ${ }^{1}$, \\ Thomas Noll ${ }^{1}$, and Florian Zuleger ${ }^{2}$ \\ 1 Software Modeling and Verification Group, \\ RWTH Aachen University, Germany \\ 2 TU Wien, Austria
}

\begin{abstract}
We introduce heap automata, a formalism for automatic reasoning about robustness properties of the symbolic heap fragment of separation logic with user-defined inductive predicates. Robustness properties, such as satisfiability, reachability, and acyclicity, are important for a wide range of reasoning tasks in automated program analysis and verification based on separation logic. Previously, such properties have appeared in many places in the separation logic literature, but have not been studied in a systematic manner. In this paper, we develop an algorithmic framework based on heap automata that allows us to derive asymptotically optimal decision procedures for a wide range of robustness properties in a uniform way.

We implemented a protoype of our framework and obtained promising results for all of the aforementioned robustness properties.

Further, we demonstrate the applicability of heap automata beyond robustness properties. We apply our algorithmic framework to the model checking and the entailment problem for symbolic-heap separation logic.
\end{abstract}

\section{Introduction}

Separation logic (SL) [40] is a popular formalism for Hoare-style verification of imperative, heap-manipulating programs. While its symbolic heap fragment originally emerged as an idiomatic form of assertions that occur naturally in hand-written proofs $[37,5,4]$, a variety of program analyses based on symbolicheap separation logic have been developed $[5,2,16,31,36,9,23]$. Consequently, it now serves as formal basis for a multitude of automated verification tools, such as $[6,15,17,21,29,39,32,8]$, capable of proving complex properties of a program's heap, such as memory safety, for large code bases $[16,15]$. These tools typically rely on systems of inductive predicate definitions (SID) to specify the shape of data structures employed by a program, such as trees and linked lists. Originally, separation logic tools implemented highly-specialized procedures for such fixed SIDs. As this limits their applicability, there is an ongoing trend to support custom SIDs that are either defined manually $[29,17]$ or even automatically generated. The latter may, for example, be obtained from the tool CABER [12]. 
Robustness properties Allowing for arbitrary SIDs, however, raises various questions about their robustness. A user-defined or auto-generated SID might, for example, be inconsistent, introduce unallocated logical variables, specify data structures that contain undesired cycles, or produce garbage, i.e., parts of the heap that are unreachable from any program variable. Accidentally introducing such properties into specifications can have a negative impact on performance, completeness, and even soundness of the employed verification algorithms:

- Brotherston et al. [11] point out that tools might waste time on inconsistent scenarios due to unsatisfiability of specifications.

- The absence of unallocated logical variables, also known as establishment, is required by the approach of Iosif et al. [27,28] to obtain a decidable fragment of symbolic heaps.

- Other verification approaches, such as the one by Habermehl et al. [24,25], assume that no garbage is introduced by data structure specifications.

- During program analysis and verification, questions such as reachability, acyclicity and garbage-freedom arise depending on the properties of interest. For example, as argued by Zanardini and Genaim [41], acyclicity of the heap is crucial in automated termination proofs.

Being able to check such robustness properties of custom SIDs is thus crucial (1) in debugging of separation-logic specifications prior to program analysis and (2) in the program analyses themselves. So far, however, all of the above properties have either been addressed individually or not systematically at all. For example, satisfiability is studied in detail by Brotherston et al. [11], whereas establishment is often addressed with ad-hoc solutions [27,24].

Several reasoning tasks arise in the context of robustness properties. As a motivation, consider the problem of acyclicity. If our program analysis requires acyclicity, we would like to decide whether all interpretations of a symbolic heap are acyclic; if not, to find out how cycles can be introduced into the heap (counterexample generation); and, finally, to be able to generate a new SID that does guarantee acyclicity (called refinement below). A systematic treatment of robustness properties should cover these reasoning tasks in general, not just for the problem of acyclicity.

Problem statement We would like to develop a framework that enables:

1. Decision procedures for robustness properties. In program analysis, we generally deal with symbolic heaps that reference SIDs specifying unbounded data structures and thus usually have infinitely many interpretations. We need to be able to decide whether all, or some, of these infinitely many interpretations are guaranteed to satisfy a given robustness property.

2. Generation of counterexamples that violate a desired property.

3. Refinement of SIDs to automatically generate a new SID that respects a given robustness property.

4. Automatic combination of decision procedures to derive decision procedures for complex robustness properties from simpler ingredients. 
Motivating example: Inductive reasoning about robustness properties The key insight underlying our solution to the above problems is that many properties of symbolic heaps can be decided iteratively by inductive reasoning. To motivate our approach, we illustrate this reasoning process with a concrete example. Consider an SID for acyclic singly-linked list segments with head $x$ and tail $y$ :

$$
\operatorname{sll} \Leftarrow \operatorname{emp}:\{x=y\} \quad \operatorname{sll} \Leftarrow \exists u . x \mapsto u * \operatorname{sll}(u y):\{x \neq y\} .
$$

The two rules of the SID define a case distinction: A list is either empty, but then the first and the last element are the same; or, the first element has a successor $u$ (specified by the points-to assertion $x \mapsto u$ ), which in turn is at the head of a (shorter) singly-linked list segment, sll $(u y)$. The inequality in the second rule guarantees that there is no cyclic model. Now, consider the following symbolic heap with predicate calls to sll: $\varphi=\exists x y z . \operatorname{sll}(x z) * z \mapsto y * \operatorname{sll}(y x)$, which might appear as an assertion during program analysis. Say our program analysis depends on the acyclicity of $\varphi$, so we need to determine whether $\varphi$ is acyclic. We can do so by inductive reasoning as follows.

- We analyze the call $\operatorname{sll}(x z)$, the first list segment in the symbolic heap $\varphi$. If it is interpreted by the right-hand side of the first rule of the SID from above, then there is no cycle in $\operatorname{sll}(x z)$ and $z$ is reachable from $x$.

- If we already know for a call $\operatorname{sll}(u z)$ that all of its models are acyclic structures and that $z$ is reachable from $u$, then $z$ is also reachable from $x$ in the symbolic heap $\exists u . x \mapsto u * \operatorname{sll}(u z):\{x \neq z\}$ obtained by the second rule of the SID. Since our SID does not introduce dangling pointers, we also know that there is still no cycle.

- By induction, $\operatorname{sll}(x z)$ is thus acyclic and $z$ is reachable from $x$.

- Likewise, $\operatorname{sll}(y x)$ is acyclic and $x$ is reachable from $y$.

- Now, based on the information we discovered for $\operatorname{sll}(x z)$ and $\operatorname{sll}(y x)$, we examine $\varphi$ and conclude that it is cyclic, as $z$ is reachable from $x, y$ is reachable from $z$, and $x$ is reachable from $y$. Crucially, we reason inductively and thus do not re-examine the list segments to arrive at our conclusion.

In summary, we examine a symbolic heap and corresponding SID bottom-up, starting from the non-recursive base case. Moreover, at each stage of this analysis, we remember a fixed amount of information - namely what we discover about reachability between parameters and acyclicity of every symbolic heap we examine. Similar inductive constructions are defined explicitly for various robustness properties throughout the separation logic literature [11,13,27]. Our aim is to generalize such manual constructions following an automata-theoretic approach: We introduce automata that operate on symbolic heaps and store the relevant information of each symbolic heap they examine in their state space. Whenever such an automaton comes across a predicate that it has already analyzed, it can simply replace the predicate with the information that is encoded in the corresponding state. In other words, our automata recognize robustness properties in a compositional way by exploiting the inductive structure inherent in the SIDs.

Systematic reasoning about robustness properties Our novel automaton model, heap automata, works directly on the structure of symbolic heaps as outlined in 
the example, and can be applied to all the problems introduced before. In particular, heap automata enable automatic refinement of SIDs and enjoy a variety of closure properties through which we can derive counterexample generation as well as decision procedures for various robustness properties - including satisfiability, establishment, reachability, garbage-freedom, and acyclicity.

Our approach can thus be seen as an algorithmic framework for deciding a wide range of robustness properties of symbolic heaps. Furthermore, we show asymptotically optimal complexity of our automata-based decision procedures in a uniform way. By enabling this systematic approach to reasoning about robustness, our framework generalizes prior work that studied single robustness properties in isolation, such as the work by Brotherston et al. [11,13].

As a natural byproduct of our automata-based approach, we also derive decision procedures for the model-checking problem, which was recently studied, and proven to be ExpTIME-complete in general, by Brotherston et al. [13]. This makes it possible to apply our framework to run-time verification - a setting in which robustness properties are of particular importance [35,29,13].

Entailment checking with heap automata Finally, we also address the entailment problem. In Hoare-style program analysis, decision procedures for the entailment problem become essential to discharge implications between assertions, as required, for example, by the rule of consequence [26]. Because of this central role in verification, there is an extensive body of research on decision procedures for entailment; see, for example [3,10,14,27,28,33,38,22]. Antonopoulos et al. [1] study the complexity of the entailment problem and show that it is undecidable in general, and already ExPTime-hard for SIDs specifying sets of trees.

We use heap automata to check entailment between determined symbolic heaps. Intuitively, determinedness is a strong form of the establishment property guaranteeing that two variables are either equal or unequal in every model. Unlike other decision procedures [27,28,3], our approach does not impose syntactic restrictions on the symbolic heap under consideration but merely requires that suitable heap automata for the predicates on the right-hand side of the entailment are provided. In particular, we show how to obtain ExPTime decision procedures from such heap automata - which exist for highly non-trivial SIDs. If desired, additional syntactic restrictions can be integrated seamlessly into our approach to boost our algorithms' performance.

Contributions Our main contributions can be summarized as follows.

- We introduce heap automata, a novel automaton model operating directly on symbolic heaps. We prove that heap automata enjoy various useful closure properties. Besides union, intersection and complement, they are closed under the conjunction with pure formulas, allowing the construction of complex heap automata from simple ones.

- We develop a powerful algorithmic framework for automated reasoning about and debugging of symbolic heaps with inductive predicate definitions based on heap automata. 
- We show that key robustness properties, such as satisfiability, establishment, reachability, garbage freedom and acyclicity, can naturally be expressed as heap automata. Moreover, the upper bounds of decision procedures obtained from our framework are shown to be optimal-i.e., ExPTime-complete - in each of these cases. Further, they enable automated refinement of SIDs to filter out (or expose) symbolic heaps with undesired properties.

- Additionally, we apply heap automata to tackle the entailment and the model checking problem for symbolic heaps. We show that if each predicate of an SID can be represented by a heap automaton, then the entailment problem for the corresponding fragment of symbolic heaps is decidable in 2-EXPTIME in general and ExpTime-complete if the maximal arity of predicates and points-to assertions is bounded. For example, our framework yields an ExPTIME decision procedure for a symbolic heap fragment capable of representing trees with linked leaves - a fragment that is out of scope of most ExPTime decision procedures known so far (cf. [3,22,28]).

- We implemented a prototype of our framework that yields promising results for all robustness properties considered in the paper.

Organization of the paper The fragment of symbolic heaps with inductive predicate definitions is briefly introduced in Section 2. Heap automata and derived decision procedures are studied in Section 3. Section 4 demonstrates that a variety of robustness properties can be checked by heap automata. We report on a prototypical implementation of our framework in Section 5. Special attention to the entailment problem is paid in Section 6. Finally, Section 7 concludes. Most proofs as well as detailed constructions are provided in the appendix for the readers convenience.

\section{Symbolic Heaps}

This section briefly introduces the symbolic heap fragment of separation logic equipped with inductive predicate definitions.

Basic Notation $\mathbb{N}$ is the set of natural numbers and $2^{S}$ is the powerset of a set $S$. $(c o) \operatorname{dom}(f)$ is the (co)domain of a (partial) function $f$. We abbreviate tuples $u_{1} \ldots u_{n}, n \geq 0$, by $\mathbf{u}$ and write $\mathbf{u}[i], 1 \leq i \leq\|\mathbf{u}\|=n$, to denote $u_{i}$, the $i$-th element of $\mathbf{u}$. By slight abuse of notation, the same symbol $\mathbf{u}$ is used for the set of all elements occurring in tuple $\mathbf{u}$. The empty tuple is $\varepsilon$ and the set of all (non-empty) tuples [of length $n \geq 0$ ] over a finite set $S$ is $S^{*}\left(S^{+}\left[S^{n}\right]\right)$. The concatenation of tuples $\mathbf{u}$ and $\mathbf{v}$ is $\mathbf{u} \mathbf{v}$.

Syntax We usually denote variables taken from Var (including a dedicated variable null) by $a, b, c, x, y, z$, etc. Moreover, let Pred be a set of predicate symbols and ar : Pred $\rightarrow \mathbb{N}$ be a function assigning each symbol its arity. Spatial formulas $\Sigma$ and pure formulas $\pi$ are given by the following grammar:

$$
\Sigma::=\operatorname{emp}|x \mapsto \mathbf{y}| \Sigma * \Sigma \quad \pi::=x=y \mid x \neq y,
$$


where $\mathbf{y}$ is a non-empty tuple of variables. Here, emp stands for the empty heap, $x \mapsto \mathbf{y}$ is a points-to assertion and $*$ is the separating conjunction. Furthermore, for $P \in$ Pred and a tuple of variables $\mathbf{y}$ of length $\operatorname{ar}(P), P \mathbf{y}$ is a predicate call. A symbolic heap $\varphi$ with variables $\operatorname{Var}(\varphi)$ and free variables $\mathbf{x}_{0} \subseteq \operatorname{Var}(\varphi)$ is a formula of the form $\varphi=\exists \mathbf{z} . \Sigma * \Gamma: \Pi, \quad \Gamma=P_{1} \mathbf{x}_{1} * \ldots * P_{m} \mathbf{x}_{m}$, where $\Sigma$ is a spatial formula, $\Gamma$ is a sequence of predicate calls and $\Pi$ is a finite set of pure formulas, each with variables from $\mathbf{x}_{0}$ and $\mathbf{z}$. This normal form, in which predicate calls and points-to assertions are never mixed, is chosen to simplify formal constructions. If an element of a symbolic heap is empty, we usually omit it to improve readability. For the same reason, we fix the notation from above and write $\mathbf{z}^{\varphi}, \mathbf{x}_{i}^{\varphi}, \Sigma^{\varphi}$ etc. to denote the respective component of symbolic heap $\varphi$ in formal constructions. Hence, $\left\|\mathbf{x}_{0}^{\varphi}\right\|$ and $\left\|\Gamma^{\varphi}\right\|$ refer to the number of free variables and the number of predicate calls of $\varphi$, respectively. We omit the superscript whenever the symbolic heap under consideration is clear from the context. If a symbolic heap $\tau$ contains no predicate calls, i.e., $\left\|\Gamma^{\tau}\right\|=0$, then $\tau$ is called reduced. Moreover, to simplify the technical development, we tacitly assume that null is a free variable that is passed to every predicate call. Thus, for each $i \in \mathbb{N}$, we write $\mathbf{x}_{i}[0]$ as a shortcut for null and treat $\mathbf{x}_{i}[0]$ as if $\mathbf{x}_{i}[0] \in \mathbf{x}_{i}{ }^{3}$

Systems of Inductive Definitions Every predicate symbol is associated with one or more symbolic heaps by a system of inductive definitions (SID). Formally, an SID is a finite set of rules of the form $P \Leftarrow \varphi$, where $\varphi$ is a symbolic heap with $\operatorname{ar}(P)=\left\|\mathbf{x}_{0}^{\varphi}\right\|$. The set of all predicate symbols occurring in SID $\Phi$ and their maximal arity are denoted by $\operatorname{Pred}(\Phi)$ and $\operatorname{ar}(\Phi)$, respectively.

Example 1. An SID specifying doubly-linked list segments is defined by:

$$
\begin{aligned}
\mathrm{dll} & \Leftarrow \mathrm{emp}:\{a=c, b=d\} \\
\mathrm{dll} & \Leftarrow \exists u . a \mapsto(u b) * \operatorname{dll}(u a c d),
\end{aligned}
$$

where $a$ corresponds to the head of the list, $b$ and $c$ represent the previous and the next list element and $d$ represents the tail of the list. For the sake of readability, we often prefer $a, b, c$, etc. as free variables in examples instead of $\mathbf{x}_{0}[1], \mathbf{x}_{0}[2], \mathbf{x}_{0}[3]$, etc. Further, the following rules specify binary trees with root $a$, leftmost leaf $b$ and successor of the rightmost leaf $c$ in which all leaves are connected by a singly-linked list from left to right.

$$
\begin{aligned}
& \text { tll } \Leftarrow a \mapsto(\text { null null } c):\{a=b\} \\
& \text { tll } \Leftarrow \exists \ell r z . a \mapsto(\ell r \text { null }) * \operatorname{tll}(\ell b z) * \operatorname{tll}(r z c) .
\end{aligned}
$$

Definition 1. We write $\mathrm{SH}$ for the set of all symbolic heaps and $\mathrm{SH}^{\Phi}$ for the set of symbolic heaps restricted to predicate symbols taken from SID $\Phi$. Moreover, given a computable function $\mathcal{C}: \mathrm{SH} \rightarrow\{0,1\}$, the set of symbolic heaps $\mathrm{SH}_{\mathcal{C}}$ is

\footnotetext{
${ }^{3}$ Since $\mathbf{x}_{i}[0]$ is just a shortcut and not a proper variable, $\left\|\mathbf{x}_{i}\right\|$ refers to the number of variables in $\mathbf{x}_{i}$ apart from $\mathbf{x}_{i}[0]$.
} 


$$
\begin{aligned}
& s, h \models_{\Phi} x \sim y \quad \Leftrightarrow s(x) \sim s(y) \text {, where } \sim \in\{=, \neq\} \\
& s, h \models_{\Phi} \mathrm{emp} \quad \Leftrightarrow \operatorname{dom}(h)=\emptyset \\
& s, h \models_{\Phi} x \mapsto \mathbf{y} \quad \Leftrightarrow \operatorname{dom}(h)=\{s(x)\} \text { and } h(s(x))=s(\mathbf{y}) \\
& s, h \models_{\Phi} P \mathbf{y} \quad \Leftrightarrow \exists \tau \in \mathbb{U}_{\Phi}(P \mathbf{y}) . s, h \models_{\emptyset} \tau \\
& s, h \models_{\Phi} \varphi * \psi \quad \Leftrightarrow \exists h_{1}, h_{2} \cdot h=h_{1} \uplus h_{2} \\
& \text { and } s, h_{1} \models_{\Phi} \varphi \text { and } s, h_{2} \models_{\Phi} \psi \\
& s, h \models_{\Phi} \exists \mathbf{z} . \Sigma * \Gamma: \Pi \quad \Leftrightarrow \exists \mathbf{v} \in \operatorname{Val}^{\|\mathbf{z}\|} . s[\mathbf{z} \mapsto \mathbf{v}], h \models_{\Phi} \Sigma * \Gamma \\
& \text { and } \forall \pi \in \Pi . s[\mathbf{z} \mapsto \mathbf{v}], h \models_{\Phi} \pi
\end{aligned}
$$

Fig. 1. Semantics of the symbolic heap fragment of separation logic with respect to an SID $\Phi$ and a state $(s, h)$.

given by $\mathrm{SH}_{\mathcal{C}} \triangleq\{\varphi \in \mathrm{SH} \mid \mathcal{C}(\varphi)=1\}$. We collect all SIDs in which every righthand side belongs to $\mathrm{SH}_{\mathcal{C}}$ in $\mathrm{SID}_{\mathcal{C}}$. To refer to the set of all reduced symbolic heaps (belonging to a set defined by $\mathcal{C})$, we write $\mathrm{RSH}\left(\mathrm{RSH}_{\mathcal{C}}\right)$.

Example 2. Let $\alpha \in \mathbb{N}$ and $\mathrm{FV}^{\leq \alpha}(\varphi) \triangleq\left\{\begin{array}{ll}1, & \left\|\mathrm{x}_{0}^{\varphi}\right\| \leq \alpha \\ 0, & \text { otherwise }\end{array}\right.$.

Clearly, $\mathrm{FV}^{\leq \alpha}$ is computable. Moreover, $\mathrm{SH}_{\mathrm{FV} \leq \alpha}$ is the set of all symbolic heaps having at most $\alpha \geq 0$ free variables.

Semantics As in a typical RAM model, we assume heaps to consist of records with a finite number of fields. Let Val denote an infinite set of values and Loc $\subseteq$ Val an infinite set of addressable locations. Moreover, we assume the existence of a special non-addressable value null $\in V a l \backslash L o c$.

A heap is a finite partial function $h: L o c \rightarrow V_{a l}{ }^{+}$mapping locations to non-empty tuples of values. We write $h_{1} \uplus h_{2}$ to denote the union of heaps $h_{1}$ and $h_{2}$ provided that $\operatorname{dom}\left(h_{1}\right) \cap \operatorname{dom}\left(h_{2}\right)=\emptyset$. Otherwise, $h_{1} \uplus h_{2}$ is undefined. Variables are interpreted by a stack, i.e., a partial function $s:$ Var $\rightarrow$ Val with $s($ null $)=$ null. Furthermore, stacks are canonically extended to tuples of variables by componentwise application. We call a stack-heap pair $(s, h)$ a state. The set of all states is States. The semantics of a symbolic heap with respect to an SID and a state is shown in Figure 1. Note that the semantics of predicate calls is explained in detail next.

Unfoldings of Predicate Calls The semantics of predicate calls is defined in terms of unfolding trees. Intuitively, an unfolding tree specifies how predicate calls are replaced by symbolic heaps according to a given SID. The resulting reduced symbolic heap obtained from an unfolding tree is consequently called an unfolding. Formally, let $\varphi=\exists \mathbf{z} . \Sigma * P_{1} \mathbf{x}_{1} * \ldots * P_{m} \mathbf{x}_{m}: \Pi$. Then a predicate call $P_{i} \mathbf{x}_{i}$ may be replaced by a reduced symbolic heap $\tau$ if $\left\|\mathbf{x}_{i}\right\|=\left\|\mathbf{x}_{0}^{\tau}\right\|$ and $\operatorname{Var}(\varphi) \cap \operatorname{Var}(\tau) \subseteq \mathbf{x}_{0}^{\tau}$. 
The result of such a replacement is

$$
\begin{aligned}
\varphi\left[P_{i} / \tau\right] \triangleq & \exists \mathbf{z} \mathbf{z}^{\tau} \cdot \Sigma * \Sigma^{\tau\left[\mathbf{x}_{0}^{\tau} / \mathbf{x}_{i}\right]} * \\
& P_{1} \mathbf{x}_{1} * \ldots * P_{i-1} \mathbf{x}_{i-1} * P_{i+1} \mathbf{x}_{i+1} * \ldots * P_{m} \mathbf{x}_{m}:\left(\Pi \cup \Pi^{\tau\left[\mathbf{x}_{0}^{\tau} / \mathbf{x}_{i}\right]}\right),
\end{aligned}
$$

where $\tau\left[\mathbf{x}_{0}^{\tau} / \mathbf{x}_{i}\right]$ denotes the substitution of each free variable of $\tau$ by the corresponding parameter of $P_{i}$.

A tree over symbolic heaps $\mathrm{SH}^{\Phi}$ is a finite partial function $t: \mathbb{N}^{*} \rightarrow \mathrm{SH}^{\Phi}$ such that $\emptyset \neq \operatorname{dom}(t) \subseteq \mathbb{N}^{*}$ is prefix-closed and for all $\mathbf{u} \in \operatorname{dom}(t)$ with $t(\mathbf{u})=\varphi$, we have $\left\{1, \ldots,\left\|\Gamma^{\varphi}\right\|\right\}=\{i \in \mathbb{N} \mid \mathbf{u} i \in \operatorname{dom}(t)\}$. The element $\varepsilon \in \operatorname{dom}(t)$ is called the root of tree $t$. Furthermore, the subtree $\left.t\right|_{\mathbf{u}}$ of $t$ with root $\mathbf{u}$ is $\left.t\right|_{\mathbf{u}}:\{\mathbf{v} \mid \mathbf{u} \mathbf{v} \in \operatorname{dom}(t)\} \rightarrow \mathrm{SH}^{\Phi}$ with $\left.t\right|_{\mathbf{u}}(\mathbf{v}) \triangleq t(\mathbf{u} \cdot \mathbf{v})$.

Definition 2. Let $\Phi \in \mathrm{SID}$ and $\varphi \in \mathrm{SH}^{\Phi}$. Then the set of unfolding trees of $\varphi$ w.r.t. $\Phi$, written $\mathbb{T}_{\Phi}(\varphi)$, is the least set that contains all trees $t$ that satisfy (1) $t(\varepsilon)=\varphi$ and (2) $\left.t\right|_{i} \in \mathbb{T}_{\Phi}\left(\psi_{i}\right)$ for each $1 \leq i \leq\left\|\Gamma^{\varphi}\right\|$, where $P_{i}^{\varphi} \Leftarrow \psi_{i} \in \Phi$.

Note that for every reduced symbolic heap $\tau$, we have $\left\|\Gamma^{\tau}\right\|=0$. Thus, $\mathbb{T}_{\Phi}(\tau)=$ $\{t\}$, where $t:\{\varepsilon\} \rightarrow\{\tau\}: \varepsilon \mapsto \tau$, forms the base case in Definition 2. Every unfolding tree $t$ specifies a reduced symbolic heap $\llbracket t \rrbracket$, which is obtained by recursively replacing predicate calls by reduced symbolic heaps:

Definition 3. The unfolding of an unfolding tree $t \in \mathbb{T}_{\Phi}(\varphi)$ is

$$
\llbracket t \rrbracket \triangleq \begin{cases}t(\varepsilon) & ,\left\|\Gamma^{t(\varepsilon)}\right\|=0 \\ t(\varepsilon)\left[P_{1} /\left.\llbracket t\right|_{1} \rrbracket, \ldots, P_{m} /\left.\llbracket t\right|_{m} \rrbracket\right] & ,\left\|\Gamma^{t(\varepsilon)}\right\|=m>0,\end{cases}
$$

where we tacitly assume that the variables $\mathbf{z}^{t(\varepsilon)}$, i.e., the existentially quantified variables in $t(\varepsilon)$, are substituted by fresh variables.

Example 3. Recall from Example 1 the two symbolic heaps $\tau$ (upper) and $\varphi$ (lower) occurring on the right-hand side of the dll predicate. Then $t:\{\varepsilon, 1\} \rightarrow$ $\{\varphi, \tau\}: \varepsilon \mapsto \varphi, 1 \mapsto \tau$ is an unfolding tree of $\varphi$. The corresponding unfolding is

$$
\llbracket t \rrbracket=\varphi\left[P_{1}^{\varphi} / \tau\right]=\exists z \cdot a \mapsto(z b) * \mathrm{emp}:\{z=c, a=d\} .
$$

Definition 4. The set of all unfoldings of a predicate call $P_{i} \mathbf{x}_{i}$ w.r.t. an $S I D$ $\Phi$ is denoted by $\mathbb{U}_{\Phi}\left(P_{i} \mathbf{x}_{i}\right)$. Analogously, the unfoldings of a symbolic heap $\varphi$ are $\mathbb{U}_{\Phi}(\varphi) \triangleq\left\{\llbracket t \rrbracket \mid t \in \mathbb{T}_{\Phi}(\varphi)\right\}$.

Then, as already depicted in Figure 1, the semantics of predicate calls requires the existence of an unfolding satisfying a given state. This semantics corresponds to a particular iteration of the frequently used semantics of predicate calls based on least fixed points (cf. [11]). Further note that applying the SL semantics to a given symbolic heap coincides with applying them to a suitable unfolding.

Lemma 1. Let $\varphi \in \mathrm{SH}^{\Phi}$. Then, for every $(s, h) \in$ States, we have

$$
s, h \models_{\Phi} \varphi \text { iff } \exists \tau \in \mathbb{U}_{\Phi}(\varphi) . s, h \models_{\emptyset} \tau .
$$

Proof. By induction on the height of unfolding trees of $\varphi$. 


\section{Heap Automata}

In this section we develop a procedure to reason about robustness properties of symbolic heaps. This procedure relies on the notion of heap automata; a device that assigns one of finitely many states to any given symbolic heap.

Definition 5. $A$ heap automaton over $\mathrm{SH}_{\mathcal{C}}$ is a tuple $\mathfrak{A}=\left(Q, \mathrm{SH}_{\mathcal{C}}, \Delta, F\right)$, where $Q$ is a finite set of states and $F \subseteq Q$ is a set of final states, respectively. Moreover, $\Delta \subseteq Q^{*} \times \mathrm{SH}_{\mathcal{C}} \times Q$ is a decidable transition relation such that $(\mathbf{q}, \varphi, p) \in \Delta$ implies that $\|\mathbf{q}\|=\left\|\Gamma^{\varphi}\right\|$. We often write $\mathbf{q} \stackrel{\varphi}{\rightarrow}{ }_{\mathfrak{A}} p$ instead of $(\mathbf{q}, \varphi, p) \in \Delta$.

A transition $\mathbf{q} \stackrel{\varphi}{\rightarrow}_{\mathfrak{A}} p$ takes a symbolic heap $\varphi$ and an input state $q_{i}$ for every predicate call $P_{i}$ of $\varphi$ - collected in the tuple $\mathbf{q}$-and assigns an output state $p$ to $\varphi$. Thus, the intuition behind a transition is that $\varphi$ has a property encoded by state $p$ if every predicate call $P_{i}$ of $\varphi$ is replaced by a reduced symbolic heap $\tau_{i}$ that has a property encoded by state $\mathbf{q}[i]$.

Note that every heap automaton $\mathfrak{A}$ assigns a state $p$ to a reduced symbolic heap $\tau$ within a single transition of the form $\varepsilon \stackrel{\tau}{\rightarrow}_{\mathfrak{A}} p$. Alternatively, $\mathfrak{A}$ may process a corresponding unfolding tree $t$ with $\llbracket t \rrbracket=\tau$. In this case, $\mathfrak{A}$ proceeds similarly to the compositional construction of unfoldings (see Definition 3). However, instead of replacing every predicate call $P_{i}$ of the symbolic heap $t(\varepsilon)$ at the root of $t$ by an unfolding $\left.\llbracket t\right|_{i} \rrbracket$ of a subtree of $t, \mathfrak{A}$ uses states to keep track of the properties of these unfolded subtrees. Consequently, $\mathfrak{A}$ assigns a state $p$ to the symbolic heap $t(\varepsilon)$ if $\left(q_{1}, \ldots, q_{m}\right) \stackrel{t(\varepsilon)}{\longrightarrow}_{\mathfrak{A}} p$ holds, where for each $1 \leq i \leq m$, $q_{i}$ is the state assigned to the unfolding of subtree $\left.t\right|_{i}$, i.e., there is a transition $\varepsilon \stackrel{\left.\llbracket t\right|_{i} \rrbracket_{\longrightarrow}}{\longrightarrow} q_{i}$. It is then natural to require that $p$ should coincide with the state assigned directly to the unfolding $\llbracket t \rrbracket$, i.e., $\varepsilon \stackrel{\llbracket t \rrbracket}{\longrightarrow}_{\mathfrak{A}} p$. Hence, we require all heap automata considered in this paper to satisfy a compositionality property.

Definition 6. A heap automaton $\mathfrak{A}=\left(Q, \mathrm{SH}_{\mathcal{C}}, \Delta, F\right)$ is compositional if for every $p \in Q$, every $\varphi \in \mathrm{SH}_{\mathcal{C}}$ with $m \geq 0$ predicate calls $\Gamma^{\varphi}=P_{1} \mathbf{x}_{1} * \ldots * P_{m} \mathbf{x}_{m}$, and all reduced symbolic heaps $\tau_{1}, \ldots, \tau_{m} \in \mathrm{RSH}_{\mathcal{C}}$, we have:

$$
\begin{gathered}
\exists \mathbf{q} \in Q^{m} .(\mathbf{q}, \varphi, p) \in \Delta \text { and } \bigwedge_{1 \leq i \leq m}\left(\varepsilon, \tau_{i}, \mathbf{q}[i]\right) \in \Delta \\
\text { if and only if } \\
\left(\varepsilon, \varphi\left[P_{1} / \tau_{1}, \ldots, P_{m} / \tau_{m}\right], p\right) \in \Delta .
\end{gathered}
$$

Due to the compositionality property, we can safely define the language $L(\mathfrak{A})$ accepted by a heap automaton $\mathfrak{A}$ as the set of all reduced symbolic heaps that are assigned a final state, i.e., $L(\mathfrak{A}) \triangleq\left\{\tau \in \mathrm{RSH}_{\mathcal{C}} \mid \exists q \in F . \varepsilon \stackrel{\tau}{\rightarrow}_{\mathfrak{A}} q\right\}$.

Example 4. Given a symbolic heap $\varphi$, let $\left|\Sigma^{\varphi}\right|$ denote the number of pointsto assertions in $\varphi$. As a running example, we consider a heap automaton $\mathfrak{A}=$ $(\{0,1\}, \mathrm{SH}, \Delta,\{1\})$, where $\Delta$ is given by

$$
\mathbf{q} \stackrel{\varphi}{\rightarrow}_{\mathfrak{A}} p \text { iff } p= \begin{cases}1, & \text { if }\left|\Sigma^{\varphi}\right|+\sum_{i=1}^{\|\mathbf{q}\|} \mathbf{q}[i]>0 \\ 0, & \text { otherwise. }\end{cases}
$$


While $\mathfrak{A}$ is a toy example, it illustrates the compositionality property: Consider the reduced symbolic heap $\tau=\exists z$.emp $* \operatorname{emp}:\{x=z, z=y\}$. Since $\tau$ contains no points-to assertions, $\mathfrak{A}$ rejects $\tau$ in a single step, i.e., $\varepsilon \stackrel{\tau}{\rightarrow}_{\mathfrak{A}} 0 \notin\{1\}$. The compositionality property of $\mathfrak{A}$ ensures that $\mathfrak{A}$ yields the same result for every unfolding tree $t$ whose unfolding $\llbracket t \rrbracket$ is equal to $\tau$. For instance, $\tau$ is a possible unfolding of the symbolic heap $\varphi=\exists z \cdot \operatorname{sll}(x z) * \operatorname{sll}(z y)$, where $\operatorname{sll}$ is a predicate specifying singly-linked list segments as in Section 1. More precisely, if both predicates are replaced according to the rule sll $\Leftarrow$ emp : $\{x=y\}$, we obtain $\tau$ again (up to renaming of parameters as per Definition 3). In this case, $\mathfrak{A}$ rejects as before: We have $\varepsilon \stackrel{\text { emp: }\{x=y\}}{\longrightarrow} \mathfrak{A} 0$ for both base cases and $(0,0) \stackrel{\varphi}{\rightarrow}_{\mathfrak{A}} 0$ for the symbolic heap $\varphi$. By the compositionality property, this is equivalent to $\varepsilon \stackrel{\tau}{\rightarrow} \mathfrak{A} 0$. Analogously, if an sll predicate, say the first, is replaced according to the rule $\operatorname{sll} \Leftarrow \psi$, where $\psi=\exists z \cdot x \mapsto z * \operatorname{sll}(z y)$, then $0 \stackrel{\psi}{\rightarrow}_{\mathfrak{A}} 1,1 \stackrel{\psi}{\rightarrow}_{\mathfrak{A}} 1$ and $(1,0) \stackrel{\varphi}{\rightarrow}_{\mathfrak{A}} 1$ holds, i.e., $\mathfrak{A}$ accepts. In general, $L(\mathfrak{A})$ is the set of all reduced symbolic heaps that contain at least one points-to assertion.

While heap automata can be applied to check whether a single reduced symbolic heap has a property of interest, i.e., belongs to the language of a heap automaton, our main application is directed towards reasoning about infinite sets of symbolic heaps, such as all unfoldings of a symbolic heap $\varphi$. Thus, given a heap automaton $\mathfrak{A}$, we would like to answer the following questions:

1. Does there exist an unfolding of $\varphi$ that is accepted by $\mathfrak{A}$ ?

2. Are all unfoldings of $\varphi$ accepted by $\mathfrak{A}$ ?

We start with a special case of the first question in which $\varphi$ is a single predicate call. The key idea behind our corresponding decision procedure is to transform the SID $\Phi$ to filter out all unfoldings that are not accepted by $\mathfrak{A}$. One of our main results is that such a refinement is always possible.

Theorem 1 (Refinement Theorem). Let $\mathfrak{A}$ be a heap automaton over $\mathrm{SH}_{\mathcal{C}}$ and $\Phi \in \operatorname{SID}_{\mathcal{C}}$. Then one can effectively construct a refined $\Psi \in \operatorname{SID}_{\mathcal{C}}$ such that for each $P \in \operatorname{Pred}(\Phi)$, we have $\mathbb{U}_{\Psi}\left(P \mathbf{x}_{0}\right)=\mathbb{U}_{\Phi}\left(P \mathbf{x}_{0}\right) \cap L(\mathfrak{A})$.

Proof. We construct $\Psi \in \operatorname{SID}_{\mathcal{C}}$ over the predicate symbols $\operatorname{Pred}(\Psi)=(\operatorname{Pred}(\Phi) \times$ $\left.Q_{\mathfrak{A}}\right) \cup \operatorname{Pred}(\Phi)$ as follows: If $P \Leftarrow \varphi \in \Phi$ with $\Gamma^{\varphi}=P_{1} \mathbf{x}_{1} * \ldots * P_{m} \mathbf{x}_{m}, m \geq 0$, and $q_{1} \ldots q_{m} \stackrel{\varphi}{\rightarrow}_{\mathfrak{A}} q_{0}$, we add a rule to $\Psi$ in which $P$ is substituted by $\left\langle P, q_{0}\right\rangle$ and each predicate call $P_{i} \mathbf{x}_{i}$ is substituted by a call $\left\langle P_{i}, q_{i}\right\rangle \mathbf{x}_{i}$. Furthermore, for each $q \in F_{\mathfrak{A}}$, we add a rule $P \Leftarrow\langle P, q\rangle \mathbf{x}_{0}$ to $\Psi$. See Appendix A.7 for details.

Example 5. Applying the refinement theorem to the heap automaton from Example 4 and the SID from Example 1 yields a refined SID given by the rules:

$$
\begin{aligned}
\mathrm{dll} l & \Leftarrow\langle\mathrm{dll}, 1\rangle(a b c d) & \langle\mathrm{dll}, 1\rangle \Leftarrow \exists z . a \mapsto(z b) *\langle\mathrm{dll}, 0\rangle(z a c d) \\
\langle\mathrm{dll}, 0\rangle & \Leftarrow \mathrm{emp}:\{a=c, b=d\} & \langle\mathrm{dll}, 1\rangle \Leftarrow \exists z . a \mapsto(z b) *\langle\mathrm{dll}, 1\rangle(z a c d)
\end{aligned}
$$

Hence, the refined predicate dll specifies all non-empty doubly-linked lists.

To answer question (1) we then check whether the set of unfoldings of a refined SID is non-empty. This boils down to a simple reachability analysis. 


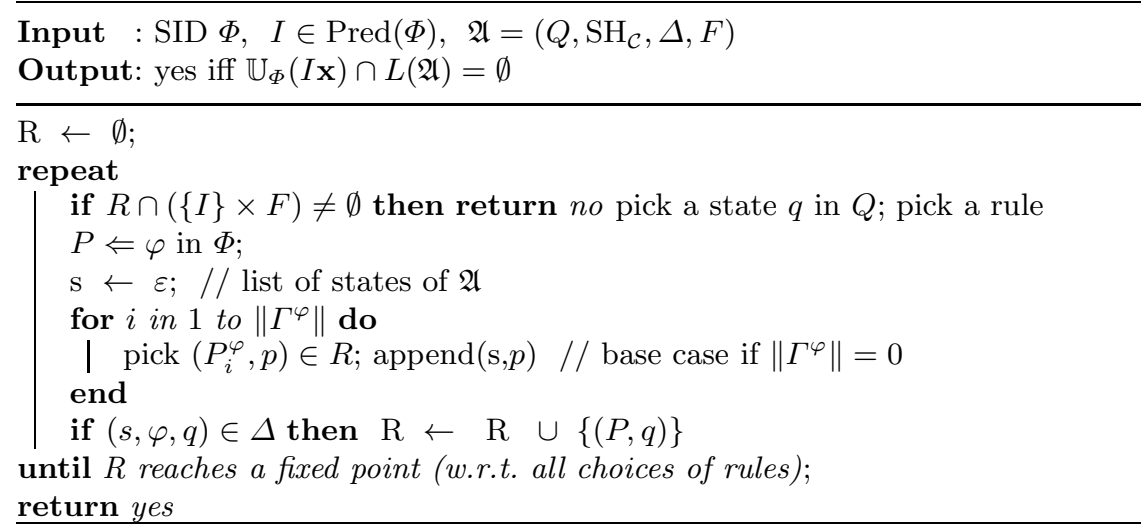

Algorithm 1: On-the-fly construction of a refined SID with emptiness check.

Lemma 2. Given an $S I D \Phi$ and a predicate symbol $P \in \operatorname{Pred}(\Phi)$, it is decidable in linear time whether the set of unfoldings of $P$ is empty, i.e., $\mathbb{U}_{\Phi}(P \mathbf{x})=\emptyset$.

Proof (sketch). It suffices to check whether the predicate $P$ lies in the least set $R$ such that (1) $I \in R$ if $I \Leftarrow \tau \in \Phi$ for some $\tau \in \operatorname{RSH}$, and (2) $I \in R$ if $I \Leftarrow \varphi \in \Phi$ and for each $P_{i}^{\varphi} \mathbf{x}_{i}^{\varphi}, 1 \leq i \leq\left\|\Gamma^{\varphi}\right\|, P_{i}^{\varphi} \in R$. The set $R$ is computable in linear time by a straightforward backward reachability analysis.

As outlined before, putting the Refinement Theorem and Lemma 2 together immediately yields a decision procedure for checking whether some unfolding of a predicate symbol $P$ is accepted by a heap automaton: Construct the refined SID and subsequently check whether the set of unfoldings of $P$ is non-empty.

To extend this result from unfoldings of single predicates to unfoldings of arbitrary symbolic heaps $\varphi$, we just add a rule $P \Leftarrow \varphi$, where $P$ is a fresh predicate symbol, and proceed as before.

Corollary 1. Let $\mathfrak{A}$ be a heap automaton over $\mathrm{SH}_{\mathcal{C}}$ and $\Phi \in \mathrm{SID}_{\mathcal{C}}$. Then, for each $\varphi \in \mathrm{SH}_{\mathcal{C}}^{\Phi}$, it is decidable whether there exists $\tau \in \mathbb{U}_{\Phi}(\varphi)$ such that $\tau \in L(\mathfrak{A})$.

The refinement and emptiness check can also be integrated: Algorithm 1 displays a simple procedure that constructs the refined SID $\Psi$ from Theorem 1 on-the-fly while checking whether its set of unfoldings is empty for a given predicate symbol. Regarding complexity, the size of a refined $\mathrm{SID}^{4}$ obtained from an $\operatorname{SID} \Phi$ and a heap automaton $\mathfrak{A}$ is bounded by $\|\Phi\| \cdot\left\|Q_{\mathfrak{A}}\right\|^{M+1}$, where $M$ is the maximal number of predicate calls occurring in any rule of $\Phi$. Thus, the aforementioned algorithm runs in time $\mathcal{O}\left(\|\Phi\| \cdot\left\|Q_{\mathfrak{A}}\right\|^{M+1} \cdot\left\|\Delta_{\mathfrak{A}}\right\|\right)$, where $\left\|\Delta_{\mathfrak{A}}\right\|$ denotes the complexity of deciding whether the transition relation $\Delta_{\mathfrak{A}}$ holds for a given tuple of states and a symbolic heap occurring in a rule of $\Phi$.

\footnotetext{
${ }^{4}$ We assume a reasonable function $\|$.$\| assigning a size to SIDs, symbolic heaps, un-$ folding trees, etc. For instance, the size $\|\Phi\|$ of an SID $\Phi$ is given by the product of its number of rules and the size of the largest symbolic heap contained in any rule.
} 
Example 6. Resuming our toy example, we check whether some unfolding of the doubly-linked list predicate dll (see Example 1) contains points-to assertions. Formally, we decide whether $\mathbb{U}_{\Phi}\left(\operatorname{dll} \mathbf{x}_{0}\right) \cap L(\mathfrak{A}) \neq \emptyset$, where $\mathfrak{A}$ is the heap automaton introduced in Example 4. Algorithm 1 first picks the rule that maps dll to the empty list segment and consequently adds $\langle$ dll, 0$\rangle$ to the set $R$ of reachable predicate-state pairs. In the next iteration, it picks the rule that maps to the non-empty list. Since $\langle\mathrm{dll} l, 0\rangle \in R, s$ is set to 0 in the do-loop. Abbreviating the body of the rule to $\varphi$, we have $(0, \varphi, 1) \in \Delta$, so the algorithm adds $\langle\mathrm{dll}, 1\rangle$ to $R$. After that, no is returned, because 1 is a final state of $\mathfrak{A}$. Hence, some unfolding of $\mathrm{dll}$ is accepted by $\mathfrak{A}$ and thus contains points-to assertions.

We now revisit question (2) from above-are all unfoldings accepted by a heap automaton?- and observe that heap automata enjoy several closure properties.

Theorem 2. Let $\mathfrak{A}$ and $\mathfrak{B}$ be heap automata over $\mathrm{SH}_{\mathcal{C}}$. Then there exist heap automat $\mathfrak{C}_{1}, \mathfrak{C}_{2}, \mathfrak{C}_{3}$ over $\mathrm{SH}_{\mathcal{C}}$ with $L\left(\mathfrak{C}_{1}\right)=L(\mathfrak{A}) \cup L(\mathfrak{B}), L\left(\mathfrak{C}_{2}\right)=L(\mathfrak{A}) \cap L(\mathfrak{B})$, and $L\left(\mathfrak{C}_{3}\right)=\mathrm{RSH}_{\mathcal{C}} \backslash L(\mathfrak{A})$, respectively. ${ }^{5}$

Then, by the equivalence $X \subseteq Y \Leftrightarrow X \cap \bar{Y}=\emptyset$ and Theorem 2, it is also decidable whether every unfolding of a symbolic heap is accepted by a heap automaton.

Corollary 2. Let $\mathfrak{A}$ be a heap automaton over $\mathrm{SH}_{\mathcal{C}}$ and $\Phi \in \operatorname{SID}_{\mathcal{C}}$. Then, for each $\varphi \in \mathrm{SH}_{\mathcal{C}}$, it is decidable whether $\mathbb{U}_{\Phi}(\varphi) \subseteq L(\mathfrak{A})$ holds.

Note that complementation of heap automata in general leads to an exponentially larger state space and exponentially higher complexity of evaluating $\Delta$. Thus, $\mathbb{U}_{\Phi}(\varphi) \subseteq L(\mathfrak{A})$ is decidable in time $\mathcal{O}\left((\|\varphi\|+\|\Phi\|) \cdot\left\|2^{Q_{\mathfrak{A}}}\right\|^{2(M+1)} \cdot\left\|\Delta_{\mathfrak{A}}\right\|\right)$. In many cases it is, however, possibly to construct smaller automata for the complement directly to obtain more efficient decision procedures. For example, this is the case for most heap automata considered in Section 4.

Apart from decision procedures, Theorem 1 enables systematic refinement of SIDs according to heap automata in order to establish desired properties. For instance, as shown in Section 4, an SID in which every unfolding is satisfiable can be constructed from any given SID. Another application of Theorem 1 is counterexample generation for systematic debugging of SIDs that are manually written as data structure specifications or even automatically generated. Such counterexamples are obtained by constructing the refined SID of the complement of a given heap automaton. Further applications are examined in the following.

Remark 1. While we focus on the well-established symbolic heap fragment of separation logic, we remark that the general reasoning principle underlying heap automata is also applicable to check robustness properties of richer fragments. For example, permissions [7] are easily integrated within our framework.

\section{A Zoo of Robustness Properties}

This section demonstrates the wide applicability of heap automata to decide and establish robustness properties of SIDs. In particular, the sets of symbolic heaps

\footnotetext{
${ }^{5}$ Formal constructions are found in Appendix A.6.
} 
informally presented in the introduction can be accepted by heap automata over the set $\mathrm{SH}_{\mathrm{FV} \leq \alpha}$ of symbolic heaps with at most $\alpha \geq 0$ free variables (cf. Example 2). Furthermore, we analyze the complexity of related decision problems. Towards a formal presentation, some terminology is needed.

Definition 7. The set of tight models of a symbolic heap $\varphi \in \mathrm{SH}^{\Phi}$ is defined as $\operatorname{Models}(\varphi) \triangleq\left\{(s, h) \in\right.$ States $\left.\mid \operatorname{dom}(s)=\mathbf{x}_{0}^{\varphi}, s, h \models_{\Phi} \varphi\right\}$.

We often consider relationships between variables that hold in every tight model of a reduced symbolic heap. Formally, let $\tau \triangleq \exists \mathbf{z} . \Sigma: \Pi \in \mathrm{RSH}$. Moreover, let $\operatorname{strip}(\tau)$ be defined as $\tau$ except that each of its variables is free, i.e., strip $(\tau) \triangleq \Sigma$ : $\Pi$. Then two variables $x, y \in \operatorname{Var}(\tau)$ are definitely (un) equal in $\tau$, written $x={ }_{\tau} y$ $\left(x \neq \neq_{\tau} y\right)$, if $s(x)=s(y)(s(x) \neq s(y))$ holds for every $(s, h) \in \operatorname{Models}(\operatorname{strip}(\tau))$. Analogously, a variable is definitely allocated if it is definitely equal to a variable occurring on the left-hand side of a points-to assertion. Thus the set of definitely allocated variables in $\tau$ is given by

$$
\operatorname{alloc}(\tau)=\{x \in \operatorname{Var}(\tau) \mid \forall(s, h) \in \operatorname{Models}(\operatorname{strip}(\tau)) . s(x) \in \operatorname{dom}(h)\} .
$$

Finally, a variable $x$ definitely points-to variable $y$ in $\tau$, written $x \mapsto_{\tau} y$, if for every $(s, h) \in \operatorname{Models}(\operatorname{strip}(\tau))$, we have $s(y) \in h(s(x))$.

Example \%. Recall the symbolic heap $\tau$ in the first rule of predicate tll from Example 1. Then alloc $(\tau)=\{a, b\}$ and neither $a={ }_{\tau} c$ nor $a \neq_{\tau} c$ holds. Further,

$$
\begin{array}{lll}
a=_{\tau} b \text { is true, } & a=_{\tau} c \text { is false, } & a \neq_{\tau} \text { null is true, } \\
a \neq_{\tau} c \text { is false, } & a \mapsto_{\tau} c \text { is true, } & c \mapsto_{\tau} a \text { is false. }
\end{array}
$$

Remark 2. All definite relationships are decidable in polynomial time. In fact, each of these relationships boils down to first adding inequalities $x \neq$ null and $x \neq y$ for every pair $x, y$ of distinct variables occurring on the left-hand side of points-to assertions to the set of pure formulas and then computing its (reflexive), symmetric (and transitive) closure with respect to $\neq($ and $=)$. Furthermore, if the closure contains a contradiction, e.g., null $\neq$ null, it is set to all pure formulas over the variables of a given reduced symbolic heap. After that, it is straightforward to decide in polynomial time whether variables are definitely allocated, (un)equal or pointing to each other.

\subsection{Tracking Equalities and Allocation}

Consider the symbolic heap $\varphi \triangleq \exists x y z \cdot P_{1}(x y) * P_{2}(y z):\{x=z\}$. Clearly, $\varphi$ is unsatisfiable if $x=y$ holds for every unfolding of $P_{1}(x y)$ and $y \neq z$ holds for every unfolding of $P_{2}(y z)$. Analogously, $\varphi$ is unsatisfiable if $x$ is allocated in every unfolding of $P_{1}(x y)$ and $z$ is allocated in every unfolding of $P_{2}(y z)$, because $x \mapsto \_* z \mapsto \_$implies $x \neq z$. This illustrates that robustness properties, such as satisfiability, require detailed knowledge about the relationships between parameters of predicate calls. Consequently, we construct a heap automaton $\mathfrak{A}_{\text {TRACK }}$ that keeps track of this knowledge. More precisely, $\mathfrak{A}_{\text {TRACK }}$ should accept those unfoldings in which it is guaranteed that 
- given a set $A \subseteq \mathbf{x}_{0}$, exactly the variables in $A$ are definitely allocated, and - exactly the (in)equalities in a given set of pure formulas $\Pi$ hold.

Towards a formal construction, we formalize the desired set of symbolic heaps.

Definition 8. Let $\alpha \in \mathbb{N}_{>0}$ and $\mathbf{x}_{0}$ be a tuple of variables with $\left\|\mathbf{x}_{0}\right\|=\alpha$. Moreover, let $A \subseteq \mathbf{x}_{0}$ and $\Pi$ be a finite set of pure formulas over $\mathbf{x}_{0}$. The tracking property $\operatorname{TRACK}(\alpha, A, \Pi)$ is the set

$$
\begin{gathered}
\left\{\tau \in \operatorname{RSH}_{F V \leq \alpha} \mid \forall i, j \cdot \mathbf{x}_{0}[i] \in A \text { iff } \mathbf{x}_{0}[i] \in \operatorname{alloc}(\tau)\right. \\
\text { and } \left.\mathbf{x}_{0}[i] \sim \mathbf{x}_{0}[j] \in \Pi \quad \text { iff } \mathbf{x}_{0}^{\tau}[i] \sim_{\tau} \mathbf{x}_{0}^{\tau}[j]\right\} .
\end{gathered}
$$

Intuitively, our heap automaton $\mathfrak{A}_{\mathrm{TRACK}}$ stores in its state space which free variables are definitely equal, unequal and allocated. Its transition relation then enforces that these stored information are correct, i.e., a transition $\mathbf{q} \stackrel{\varphi}{\rightarrow}_{\mathfrak{A}_{\text {TRAсK }}} p$ is only possible if the information stored in $p$ is consistent with $\varphi$ and with the information stored in the states $\mathbf{q}$ for the predicate calls of $\varphi$.

Formally, let $\mathbf{x}_{0}$ be a tuple of variables with $\left\|\mathbf{x}_{0}\right\|=\alpha$ and $\operatorname{Pure}\left(\mathbf{x}_{0}\right) \triangleq$ $\left.2^{\left\{\mathbf{x}_{0}[i] \sim \mathbf{x}_{0}[j]\right.} \mid 0 \leq i, j \leq \alpha, \sim \in\{=, \neq\}\right\}$ be the powerset of all pure formulas over $\mathbf{x}_{0}$. The information stored by our automaton consists of a set of free variables $B \subseteq \mathbf{x}_{0}$ and a set of pure formulas $\Lambda \in \operatorname{Pure}\left(\mathbf{x}_{0}\right)$. Now, for some unfolding $\tau$ of a symbolic heap $\varphi$, assume that $B$ is chosen as the set of all definitely allocated free variables of $\tau$. Moreover, assume $\Lambda$ is the set of all definite (in)equalities between free variables in $\tau$. We can then construct a reduced symbolic heap $\operatorname{kernel}(\varphi,(B, \Lambda))$ from $B$ and $\Lambda$ that precisely captures these relationships between free variables.

Definition 9. Let $\varphi$ be a symbolic heap, $B \subseteq \mathbf{x}_{0}$ and $\Lambda \in$ Pure $\left(\mathbf{x}_{0}\right)$. Furthermore, let $\min (B, \Lambda)=\left\{\mathbf{x}_{0}^{i} \in B \mid \neg \exists \mathbf{x}_{0}^{j} \in B \cdot j<i\right.$ and $\left.\mathbf{x}_{0}^{i}={ }_{\Lambda} \mathbf{x}_{0}^{j}\right\}$ be the set of minimal (w.r.t. to occurrence in $\mathbf{x}_{0}$ ) allocated free variables. Then

$$
\begin{gathered}
\operatorname{kernel}(\varphi,(B, \Lambda)) \triangleq \star_{\mathbf{x}_{0}[i] \in \min (B, \Lambda)} \mathbf{x}_{0}^{\varphi}[i] \mapsto \text { null }: \Lambda, \\
\text { where we write } \star_{s \in S} s \mapsto \text { null for } s_{1} \mapsto \mathbf{n u l l} * \ldots * s_{k} \mapsto \text { null, } S=\left\{s_{1}, \ldots, s_{k}\right\} .
\end{gathered}
$$

Consequently, the relationships between free variables remain unaffected if a predicate call of $\varphi$ is replaced by $\operatorname{kernel}(\varphi,(B, \Lambda))$ instead of $\tau$. Thus, $\mathfrak{A}_{\text {TRACK }}$ has one state per pair $(B, \Lambda)$. In the transition relation of $\mathfrak{A}_{\mathrm{TRACK}}$ it suffices to replace each predicate call $P \mathbf{x}_{0}$ by the corresponding symbolic heap $\operatorname{kernel}\left(P \mathbf{x}_{0},(B, \Lambda)\right)$. and check whether the current state is consistent with the resulting symbolic heap. Intuitively, a potentially large unfolding of a symbolic heap $\varphi$ with $m$ predicate calls is "compressed" into a small one that contains all necessary information about parameters of predicate calls. Here, $\mathbf{q}$ is a sequence of pairs $(B, \Lambda)$ as explained above. Formally,

Definition 10. $\mathfrak{A}_{\mathrm{TRACK}}=\left(Q, \mathrm{SH}_{F V \leq \alpha}, \Delta, F\right)$ is given by:

$$
\begin{aligned}
& Q \triangleq 2^{\mathbf{x}_{0}} \times \operatorname{Pure}\left(\mathbf{x}_{0}\right), \quad F \triangleq\{(A, \Pi)\}, \\
& \Delta: \mathbf{q} \stackrel{\varphi}{\rightarrow}_{\mathfrak{A}_{\text {тRACK }}}\left(A_{0}, \Pi_{0}\right) \text { iff } \forall x, y \in \mathbf{x}_{0} .
\end{aligned}
$$




$$
\begin{gathered}
y \in A_{0} \leftrightarrow y^{\varphi} \in \operatorname{alloc}(\operatorname{compress}(\varphi, \mathbf{q})) \\
\text { and } x \sim y \in \Pi_{0} \leftrightarrow x^{\varphi} \sim_{\operatorname{compress}(\varphi, \mathbf{q})} y^{\varphi}, \\
\operatorname{compress}(\varphi, \mathbf{q}) \triangleq \varphi\left[P_{1} / \operatorname{kernel}\left(P_{1} \mathbf{x}_{1}, \mathbf{q}[1]\right), \ldots, P_{m} / \operatorname{kernel}\left(P_{m} \mathbf{x}_{m}, \mathbf{q}[m]\right)\right],
\end{gathered}
$$

where $m=\left\|\Gamma^{\varphi}\right\|=\|\mathbf{q}\|$ is the number of predicate calls in $\varphi$ and $y^{\varphi}$ denotes the free variable of $\varphi$ corresponding to $y \in \mathbf{x}_{0}$, i.e., if $y=\mathbf{x}_{0}[i]$ then $y^{\varphi}=\mathbf{x}_{0}^{\varphi}[i]$.

Since compress $(\tau, \varepsilon)=\tau$ holds for every reduced symbolic heap $\tau$, it is straightforward to show that $L\left(\mathfrak{A}_{\mathrm{TRACK}}\right)=\operatorname{TRACK}(\alpha, A, \Pi)$. Furthermore, $\mathfrak{A}_{\mathrm{TRACK}}$ satisfies the compositionality property. A formal proof is found in Appendix A.9.1. Hence,

Lemma 3. For all $\alpha \in \mathbb{N}_{>0}$ and all sets $A \subseteq \mathbf{x}_{0}, \Pi \in \operatorname{Pure}\left(\mathbf{x}_{0}\right)$, there is a heap automaton over $\mathrm{SH}_{F V \leq \alpha}$ accepting $\operatorname{TRACK}(\alpha, A, \Pi)$.

\subsection{Satisfiability}

Tracking relationships between free variables of symbolic heaps is a useful auxiliary construction that serves as a building block in automata for more natural properties. For instance, the heap automaton $\mathfrak{A}_{\text {TRACK }}$ constructed in Definition 10 can be reused to deal with the

Satisfiability problem (SL-SAT): Given $\Phi \in \mathrm{SID}$ and $\varphi \in \mathrm{SH}^{\Phi}$, decide whether $\varphi$ is satisfiable, i.e., there exists $(s, h) \in$ States such that $s, h \models_{\Phi} \varphi$.

Theorem 3. For each $\alpha \in \mathbb{N}_{>0}$, there is a heap automaton over $\mathrm{SH}_{F V \leq \alpha}$ accepting the set $\operatorname{SAT}(\alpha) \triangleq\left\{\tau \in \mathrm{RSH}_{F V \leq \alpha} \mid \tau\right.$ is satisfiable $\}$ of all satisfiable reduced symbolic heaps with at most $\alpha$ free variables.

Proof. A heap automaton $\mathfrak{A}_{\mathrm{SAT}}$ accepting $\operatorname{SAT}(\alpha)$ is constructed as in Definition 10 except for the set of final states, which is $F \triangleq\{(A, \Pi) \mid$ null $\neq$ null $\notin$ $\Pi\}$. See Appendix A.10 for a correctness proof.

A heap automaton accepting the complement of $\operatorname{SAT}(\alpha)$ is constructed analogously by choosing $F \triangleq\{(A, \Pi) \mid$ null $\neq$ null $\in \Pi\}$. Thus, together with Corollary 1 , we obtain a decision procedure for the satisfiability problem similar to the one proposed in [11]. Regarding complexity, the heap automaton $\mathfrak{A}_{\mathrm{SAT}}$ from Definition 10 has $2^{2 \alpha^{2}+\alpha}$ states. By Remark 2, membership in $\Delta_{\mathfrak{A}_{\text {SAT }}}$ is decidable in polynomial time. Thus, by Corollary 1 , our construction yields an exponential-time decision procedure for SL-SAT. If the number of free variables $\alpha$ is bounded, an algorithm in NP is easily obtained by guessing a suitable unfolding tree of height at most $\left\|Q_{\mathfrak{A}_{\mathrm{SAT}}}\right\|$ and running $\mathfrak{A}_{\mathrm{SAT}}$ on it to check whether its unfolding is decidable (cf. Appendix A.11). This is in line with the results of Brotherston et al. [11], where the satisfiability problem is shown to be ExPTIMEcomplete in general and $\mathrm{NP}$-complete if the number of free variables is bounded. These complexity bounds even hold for the following special case [13]:

Restricted satisfiability problem (SL-RSAT) Given an SID $\Phi$ that contains no points-to assertions, and a predicate symbol $P$, decide whether $P \mathbf{x}$ is satisfiable w.r.t. $\Phi$. The complement of this problem is denoted by $\overline{\text { SL-RSAT. }}$. 


\subsection{Establishment}

A symbolic heap $\varphi$ is established if every existentially quantified variable of every unfolding of $\varphi$ is definitely equal to a free variable or definitely allocated. ${ }^{6}$ This property is natural for symbolic heaps that specify the shape of data structures; for example, the SIDs in Example 1 define sets of established symbolic heaps. Further, establishment is often required to ensure decidability of the entailment problem [27,28]. Establishment can also be checked by heap automata.

Theorem 4. For all $\alpha \in \mathbb{N}_{>0}$, there is a heap automaton over $\mathrm{SH}_{F V \leq \alpha}$ accepting the set of all established reduced symbolic heaps with at most $\alpha$ free variables:

$$
\operatorname{EST}(\alpha) \triangleq\left\{\tau \in \operatorname{RSH}_{F V \leq \alpha} \mid \forall y \in \operatorname{Var}(\tau) \cdot y \in \operatorname{alloc}(\tau) \text { or } \exists x \in \mathbf{x}_{0}^{\tau} \cdot x={ }_{\tau} y\right\}
$$

Proof. The main idea in the construction of a heap automaton $\mathfrak{A}_{\mathrm{EST}}$ for $\operatorname{EST}(\alpha)$ is to verify that every variable is definitely allocated or equal to a free variable while running $\mathfrak{A}_{\text {TRACK }}$ (see Definition 10) in parallel to keep track of the relationships between free variables. An additional flag $q \in\{0,1\}$ is attached to each state of $\mathfrak{A}_{\text {TRACK }}$ to store whether the establishment condition is already violated $(q=0)$ or holds so far $(q=1)$. Formally, $\mathfrak{A}_{\mathrm{EST}}=\left(Q, \mathrm{SH}_{\mathrm{FV} \leq \alpha}, \Delta, F\right)$, where

$$
\begin{aligned}
& Q \triangleq Q_{\mathfrak{A}_{\mathrm{TRACK}}} \times\{0,1\}, \quad F \triangleq Q_{\mathfrak{A}_{\mathrm{TRACK}}} \times\{1\} \\
& \Delta:\left(p_{1}, q_{1}\right) \ldots\left(p_{m}, q_{m}\right) \stackrel{\varphi}{\rightarrow}_{\mathfrak{A}_{\mathrm{EST}}}\left(p_{0}, q_{0}\right) \\
& \text { iff } p_{1} \ldots p_{m} \stackrel{\varphi}{\rightarrow}_{\mathfrak{A}_{\mathrm{TRACK}}} p_{0} \text { and } q_{0}=\min \left\{q_{1}, \ldots, q_{m}, \operatorname{check}\left(\varphi, p_{1} \ldots p_{m}\right)\right\} .
\end{aligned}
$$

Here, check : $\mathrm{SH}_{\mathrm{FV} \leq \alpha} \times Q_{\mathfrak{A}_{\mathrm{TRACK}}}^{*} \rightarrow\{0,1\}$ is a predicate given by

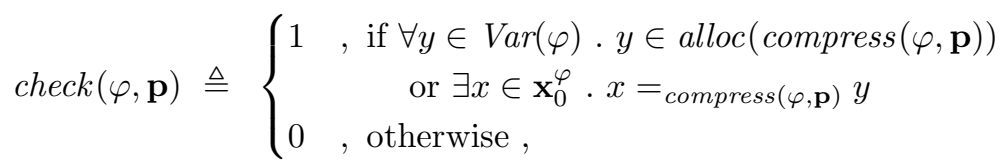

where compress $(\varphi, \mathbf{p})$ is the reduced symbolic heap obtained from the tracking property as in Definition 10. Moreover, unlike in the construction of $\mathfrak{A}_{\mathrm{TRACK}}$, we are not interested in a specific set of relationships between the pure formulas, so any state of $\mathfrak{A}_{\text {TRACK }}$ is chosen as a final state provided that predicate check could be evaluated to 1. See Appendix A.13 for a correctness proof.

Again, it suffices to swap the final- and non-final states of $\mathfrak{A}_{\mathrm{EST}}$ to obtain a heap automaton $\mathfrak{A}_{\overline{\mathrm{EST}}}$ accepting the complement of $\operatorname{EST}(\alpha)$. Thus, by Corollary 1 and Remark 2, we obtain an ExPTIME decision procedure for the

Establishment problem (SL-EST): Given an $\operatorname{SID} \Phi$ and $\varphi \in \mathrm{SH}^{\Phi}$, decide whether every $\tau \in \mathbb{U}_{\Phi}(\varphi)$ is established.

Lemma 4. $\overline{\mathrm{SL}-\mathrm{RSAT}}$ is polynomial-time reducible to SL-EST. Hence, the establishment problem SL-EST is ExPTIME-hard in general and $\mathrm{CONP}$-hard if the maximal number of free variables is bounded.

\footnotetext{
${ }^{6}$ Sometimes this property is also defined by requiring that each existentially quantified variable is "eventually allocated" [27].
} 
Proof. Let $(\Phi, P)$ be an instance of $\overline{\text { SL-RSAT }}$. Moreover, let $\varphi \triangleq \exists \mathbf{z} y$. Pz : $\left\{\mathbf{x}_{0}[1]=\right.$ null, $y \neq$ null $\}$. As $y$ is neither allocated nor occurs in $P \mathbf{z}, \varphi$ is established iff $\mathbf{x}_{0}[1]=y$ iff null $\neq$ null iff $P \mathbf{x}$ is unsatisfiable. Hence, $(\Phi, \varphi) \in \operatorname{SL}-\mathrm{EST}$ iff $(\Phi, P) \in \overline{\text { SL-RSAT. }}$. A full proof is found in Appendix A.14.

Lemma 5. SL-EST is in CONP for a bounded number of free variables $\alpha$.

Proof. Let $(\Phi, \varphi)$ be an instance of SL-EST, $N=\|\Phi\|+\|\varphi\|$, and $M \leq N$ be the maximal number of predicate calls occurring in $\varphi$ and any rule of $\Phi$. Moreover, let $\mathfrak{A}_{\overline{\mathrm{EST}}}$ be a heap automaton accepting $\overline{\operatorname{EST}(\alpha)}$ - the complement of $\operatorname{EST}(\alpha)$ (cf. Theorem 4). Since $\alpha$ is bounded by a constant, so is the number of states of $\mathfrak{A}_{\overline{\mathrm{EST}}}$, namely $\left\|Q_{\mathfrak{A}_{\overline{\mathrm{EST}}}}\right\| \leq k=2^{2 \alpha^{2}+\alpha+1}$. Now, let $\mathbb{T}_{\Phi}(\varphi) \leq k$ denote the set of all unfolding trees $t \in \mathbb{T}_{\Phi}(\varphi)$ of height at most $k$. Clearly, each of these trees is of size $\|t\| \leq M^{k} \leq N^{k}$, i.e., polynomial in $N$. Moreover, let $\omega: \operatorname{dom}(t) \rightarrow Q_{\mathfrak{A}_{\overline{\mathrm{EST}}}}$ be a function mapping each node of $t$ to a state of $\mathfrak{A}_{\overline{\mathrm{EST}}}$. Again, $\omega$ is of size polynomial in $N$; as such $\|\omega\| \leq k \cdot N^{k}$. Let $\Omega_{t}$ denote the set of all of these functions $\omega$ for a given unfolding tree $t$ with $\omega(\varepsilon) \in F_{\mathfrak{A}_{\overline{\mathrm{EST}}}}$. Given an unfolding tree $t \in \mathbb{T}_{\Phi}(\varphi) \leq k$ and $\omega \in \Omega_{t}$, we can easily decide whether $\varepsilon \stackrel{\llbracket t \rrbracket}{\longrightarrow} \mathfrak{A}_{\overline{\mathrm{EST}}} \omega(\varepsilon)$ holds: For each $u, u 1, \ldots$, un $\in \operatorname{dom}(t), u(n+1) \notin \operatorname{dom}(t), n \geq 0$, it suffices to check whether $\omega(u 1) \ldots \omega(u n) \stackrel{t(u)}{\longrightarrow} \mathfrak{A}_{\overline{\mathrm{EST}}} \omega(u)$. Since, by Remark 2, each of these checks can be performed in time polynomial in $N$ the whole procedure is feasible in polynomial time. We now show that $(\Phi, \varphi) \in \operatorname{SL}-\mathrm{EST}$ if and only if

$$
\forall t \in \mathbb{T}_{\Phi}(\varphi)^{\leq k} . \forall \omega \in \Omega_{t} \cdot \operatorname{not} \varepsilon \stackrel{\llbracket t \rrbracket}{\longrightarrow}_{\mathfrak{A}_{\overline{\mathrm{EST}}}} \omega(\varepsilon) .
$$

Since each $t \in \mathbb{T}_{\Phi}(\varphi)$ and each $\omega \in \Omega_{t}$ is of size polynomial in $N$, this is equivalent to SL-EST being in CONP. To complete the proof, note that $\mathbb{U}_{\Phi}(\varphi) \subseteq \operatorname{EST}(\alpha)$ holds iff $\llbracket t \rrbracket \notin \overline{\operatorname{EST}(\alpha)}$ for each $t \in \mathbb{T}_{\Phi}(\varphi)$. Furthermore, by a standard pumping argument, it suffices to consider trees in $\mathbb{T}_{\Phi}(\varphi)^{\leq k}$ : If there exists a taller tree $t$ with $\llbracket t \rrbracket \in \overline{\operatorname{EST}(\alpha)}$ then there is some path of length greater $k$ in $t$ on which two nodes are assigned the same state by a function $\omega \in \Omega_{t}$ proving membership of $t$ in $\overline{\operatorname{EST}(\alpha)}$. This path can be shortened to obtain a tree of smaller height.

Putting upper and lower bounds together, we conclude:

Theorem 5. SL-EST is EXPTIME-complete in general and CONP-complete if the number of free variables $\alpha$ is bounded.

\subsection{Reachability}

Another family of robustness properties is based on reachability questions, e.g., "is every location of every model of a symbolic heap reachable from the location of a program variable?" or "is every model of a symbolic heap acyclic?". For established SIDs, heap automata accepting these properties are an extension of the tracking automaton introduced in Definition 10.

More precisely, a variable $y$ is definitely reachable from $x$ in $\tau \in \mathrm{RSH}$, written $x \rightsquigarrow_{\tau} y$, if and only if $x \mapsto_{\tau} y$ or there exists a $z \in \operatorname{Var}(\tau)$ such that $x \mapsto_{\tau} z$ 
and $z \rightsquigarrow_{\tau} y \cdot{ }^{7}$ Note that we define reachability to be transitive, but not reflexive. As for the other definite relationships between variables, definite reachability is computable in polynomial time for reduced symbolic heaps, e.g., by performing a depth-first search on the definite points-to relation $\mapsto_{\tau}$. Note that our notion of reachability does not take variables into account that are only reachable from one another in some models of a reduced symbolic heap. For example, consider the symbolic heap $\tau=x \mapsto y * z \mapsto$ null. Then $x \rightsquigarrow_{\tau} z$ does not hold, but there exists a model $(s, h)$ with $s(z)=s(y) \in h(s(x))$. Thus, reachability introduced by unallocated variables is not detected. However, the existence (or absence) of such variables can be checked first due to Theorem 4 .

Theorem 6. Let $\alpha \in \mathbb{N}_{>0}$ and $R \subseteq \mathbf{x}_{0} \times \mathbf{x}_{0}$ be a binary relation over the variables $\mathbf{x}_{0}$ with $\left\|\mathbf{x}_{0}\right\|=\alpha$. Then the reachability property $\operatorname{REACH}(\alpha, R)$, given by the set $\left\{\tau \in \mathrm{RSH}_{F V \leq \alpha} \mid \forall i, j .\left(\mathbf{x}_{0}[i], \mathbf{x}_{0}[j]\right) \in R\right.$ iff $\left.\mathbf{x}_{0}^{\tau}[i] \rightsquigarrow_{\tau} \mathbf{x}_{0}^{\tau}[j]\right\}$, can be accepted by a heap automaton over $\mathrm{SH}_{F V \leq \alpha}$.

Proof (sketch). A heap automaton $\mathfrak{A}_{\text {REACH }}$ accepting $\operatorname{REACH}(\alpha, R)$ is constructed similarly to the heap automaton $\mathfrak{A}_{\mathrm{TRACK}}$ introduced in Definition 10 . The main difference is that $\mathfrak{A}_{\text {REACH }}$ additionally stores a binary relation $S \subseteq \mathbf{x}_{0} \times \mathbf{x}_{0}$ in its state space to remember which free variables are reachable from one another. Correspondingly, we adapt Definition 9 as follows:

$$
\operatorname{kernel}(\varphi,(B, \Lambda, S)) \triangleq \exists z \cdot \star_{\min (B, \Lambda)} \mathbf{x}_{0}^{\varphi}[i] \mapsto\left(\mathbf{v}_{i}\right): \Lambda,
$$

where $z$ is a fresh variable and $\mathbf{v}_{i}[j] \triangleq \mathbf{x}_{0}^{\varphi}[j]$ if $(i, j) \in S$ and $\mathbf{v}_{i}[j] \triangleq z$, otherwise. The other parameters $\varphi, B, \Lambda$ are the same as in Definition 10. Note that the additional variable $z$ is needed to deal with allocated free variables that cannot reach any other free variable, including null. Moreover, the set of final states is $F_{\mathfrak{A}_{\text {REACH }}}=Q_{\mathfrak{A}_{\text {TRACK }}} \times\{R\}$. Correctness of this encoding is verified in the transition relation. Hence, the transition relation of $\mathfrak{A}_{\text {REACH }}$ extends the transition relation of $\mathfrak{A}_{\mathrm{TRACK}}$ by the requirement $(x, y) \in S$ iff $x^{\varphi} \rightsquigarrow \operatorname{compress}(\varphi, \mathbf{p}) y^{\varphi}$ for every pair of free variables $x, y \in \mathbf{x}_{0}$. Here, $\operatorname{compress}(\varphi, \mathbf{p})$ is defined as in Definition 10 except that the new encoding $\operatorname{kernel}\left(P_{i} \mathbf{x}_{i}, \mathbf{q}[i]\right)$ from above is used. Since compress $(\tau, \varepsilon)=\tau$ holds for every reduced symbolic heap $\tau$, it is straightforward to verify that $L\left(\mathfrak{A}_{\text {REACH }}\right)=\operatorname{REACH}(\alpha)$. Further details are found in Appendix A.15.

Furthermore, we consider the related

Reachability problem (SL-REACH): Given an SID $\Phi, \varphi \in \mathrm{SH}^{\Phi}$ with $\alpha=$ $\left\|\mathbf{x}_{0}^{\varphi}\right\|$ and variables $x, y \in \mathbf{x}_{0}^{\varphi}$, decide whether $x \rightsquigarrow_{\tau} y$ holds for all $\tau \in \mathbb{U}_{\Phi}(\varphi)$.

Theorem 7. The decision problem SL-REACH is ExPTIME-complete in general and $\mathrm{CONP}$-complete if the number of free variables is bounded.

Proof. Membership in ExPTime follows from our upper bound derived for Algorithm 1, the size of the state space of $\mathfrak{A}_{\text {REACH }}$, which is exponential in $\alpha$, and Remark 2. If $\alpha$ is bounded, membership in CONP is shown analogously to Lemma 5 .

\footnotetext{
$\overline{7}$ The definite points-to relation $\mapsto_{\tau}$ was defined at the beginning of Section 4 .
} 
Lower bounds are shown by reducing SL-RSAT to SL-REACH. Formally, let $(\Phi, P)$ be an instance of SL-RSAT. Moreover, let $\varphi \triangleq \exists \mathbf{z} \cdot \mathbf{x}_{0}[1] \mapsto$ null $*$ $P \mathbf{z}:\left\{\mathbf{x}_{0}[2] \neq\right.$ null $\}$. As $\mathbf{x}_{0}[2]$ is neither allocated nor null, $\mathbf{x}_{0}[2]$ is not definitely reachable from $\mathbf{x}_{0}[1]$ in any model of $\varphi$. Hence $\left(\Phi, \varphi, \mathbf{x}_{0}[1], \mathbf{x}_{0}[2]\right) \in \mathrm{SL}-\mathrm{REACH}$ iff $P$ is unsatisfiable. A detailed proof is found in Appendix A.16.

\subsection{Garbage-Freedom}

Like the tracking automaton $\mathfrak{A}_{\mathrm{TRACK}}$, the automaton $\mathfrak{A}_{\mathrm{REACH}}$ is a useful ingredient in the construction of more complex heap automata. For instance, such an automaton can easily be modified to check whether a symbolic heap is garbage-free, i.e., whether every existentially quantified variable is reachable from some program variable. Garbage-freedom is a natural requirement if SIDs represent data structure specifications. For instance, the SIDs in Example 1 are garbage-free. Furthermore, this property is needed by the approach of Habermehl et al. [25].

Lemma 6. For each $\alpha \in \mathbb{N}_{>0}$, the set $\operatorname{GFREE}(\alpha)$, given by

$$
\left\{\tau \in \operatorname{RSH}_{F V \leq \alpha} \mid \forall y \in \operatorname{Var}(\tau) . \exists x \in \mathbf{x}_{0}^{\tau} \cdot x={ }_{\tau} y \text { or } x \rightsquigarrow_{\tau} y\right\},
$$

of garbage-free symbolic heaps can be accepted by a heap automaton over $\mathrm{SH}_{F V \leq \alpha}$.

Proof (sketch). A heap automaton $\mathfrak{A}_{\text {GFREE }}$ accepting GFREE $(\alpha)$ is constructed similarly to the heap automaton $\mathfrak{A}_{\mathrm{EST}}$ introduced in the proof of Theorem 4 . The main difference is that heap automaton $\mathfrak{A}_{\text {REACH }}$ is used instead of $\mathfrak{A}_{\text {TRACK}}$. Furthermore, the predicate check $: \mathrm{SH}_{\mathrm{FV} \leq \alpha} \times Q_{\mathfrak{A}_{\mathrm{RE}} \text { Сс }}^{*} \rightarrow\{0,1\}$ is redefined to verify that every variable of a symbolic heap $\varphi$ is established in $\operatorname{compress}(\varphi, \mathbf{p})$, where $\operatorname{compress}(\varphi, \mathbf{p})$ is the same as in the construction of $\mathfrak{A}_{\text {REACH }}$ (see Theorem 6):

$$
\operatorname{check}(\varphi, \mathbf{p}) \triangleq\left\{\begin{aligned}
1, & \text { if } \forall y \in \operatorname{Var}(\varphi) \cdot \exists x \in \mathbf{x}_{0}^{\varphi} . \\
\quad x=\operatorname{compress}(\varphi, \mathbf{p}) & y \text { or } x \rightsquigarrow \operatorname{compress}(\varphi, \mathbf{p}) y \\
0, & \text { otherwise },
\end{aligned}\right.
$$

Since compress $(\tau, \varepsilon)=\tau$ holds for every reduced symbolic heap $\tau$, it is straightforward that $L\left(\mathfrak{A}_{\mathrm{GFREE}}\right)=\operatorname{GFREE}(\alpha)$. A proof is found in Appendix A.17.

To guarantee that symbolic heaps are garbage-free, we solve the

Garbage-freedom problem (SL-GF): Given an SID $\Phi$ and $\varphi \in \mathrm{SH}^{\Phi}$, decide whether every $\tau \in \mathbb{U}_{\Phi}(\varphi)$ is garbage-free, i.e., $\tau \in \operatorname{GFREE}(\alpha)$ for some $\alpha \in \mathbb{N}$.

Theorem 8. SL-GF is EXPTIME-complete in general and CONP-complete if the number of free variables $\alpha$ is bounded.

Proof. Similar to Theorem 5; see Appendix A.18. 


\subsection{Acyclicity}

Automatic termination proofs of programs frequently rely on the acyclicity of the data structures used, i.e., they assume that no variable is reachable from itself. In fact, Zanardini and Genaim [41] claim that "proving termination needs acyclicity, unless program-specific or non-automated reasoning is performed."

Lemma 7. For each $\alpha \in \mathbb{N}_{>0}$, the set of all weakly acyclic symbolic heaps

$$
\operatorname{ACYCLIC}(\alpha) \triangleq\left\{\tau \in \operatorname{RSH}_{F V \leq \alpha} \mid \text { null } \neq_{\tau} \text { null } \text { or } \forall x \in \operatorname{Var}(\tau) \text {. not } x \rightsquigarrow_{\tau} x\right\}
$$

can be accepted by a heap automaton over $\mathrm{SH}_{F V \leq \alpha}$.

Here, the condition null $\neq_{\tau}$ null ensures that an unsatisfiable reduced symbolic heap is considered weakly acyclic. Further, note that our notion of acyclicity is weak in the sense that dangling pointers may introduce cyclic models that are not considered. For example, $\exists z \cdot x \mapsto z$ is weakly acyclic, but contains cyclic models if $x$ and $z$ are aliases. However, weak acyclicity coincides with the absence of cyclic models for established SIDs - a property considered in Section 4.3.

Proof (sketch). A heap automaton $\mathfrak{A}_{\mathrm{ACYCLIC}}$ for the set of all weakly acyclic reduced symbolic heaps is constructed analogously to the heap automaton $\mathfrak{A}_{\text {GFREE }}$ in the proof of Lemma 6 . The main difference is the predicate check: $\mathrm{SH}_{\mathrm{FV} \leq \alpha} \times$ $Q_{\mathfrak{R}_{\mathrm{REACH}}}^{*} \rightarrow\{0,1\}$, which now checks whether a symbolic heap is weakly acyclic:

$$
\operatorname{check}(\varphi, \mathbf{p}) \triangleq \begin{cases}1 & , \text { if } \forall y \in \operatorname{Var}(\varphi) \cdot \operatorname{not} x \rightsquigarrow \operatorname{compress}(\varphi, \mathbf{p}) x \\ 0 & , \text { otherwise. }\end{cases}
$$

Moreover, the set of final states $F_{\mathfrak{A}_{\text {AcYcLIc }}}$ is chosen such that accepted symbolic heaps are unsatisfiable or $\operatorname{check}(\varphi, \mathbf{p})=1$. See Appendix A.19 for details.

For example, the symbolic heap sll $\mathbf{x}_{0}$ is weakly acyclic, but dll $\mathbf{x}_{0}$ (cf. Example 1) is not. In general, we are interested in the

Acyclicity problem (SL-AC): Given an SID $\Phi$ and $\varphi \in \mathrm{SH}^{\Phi}$, decide whether every $\tau \in \mathbb{U}_{\Phi}(\varphi)$ is weakly acyclic, i.e., $\tau \in \operatorname{ACYCLIC}(\alpha)$ for some $\alpha \in \mathbb{N}$.

Theorem 9. SL-AC is ExPTIME-complete in general and CONP-complete if the number of free variables $\alpha$ is bounded.

Proof. Similar to the proof of Theorem 5. For lower bounds, we show that $\overline{\text { SL-RSAT }}$ is reducible to SL-AC. Let $(\Phi, P)$ be an instance of SL-RSAT. Moreover, let $\varphi=\exists \mathbf{z} \cdot \mathbf{x}_{0}[1] \mapsto\left(\mathbf{x}_{0}[1]\right) * P \mathbf{z}$. Since $\mathbf{x}_{0}[1]$ is definitely reachable from itself, $\varphi$ is weakly acyclic iff $P$ is unsatisfiable. Thus, $(\Phi, \varphi) \in$ SL-AC iff $(\Phi, P) \in \overline{\mathrm{SL}-\mathrm{RSAT}}$. See Appendix A.20 for details. 


\section{Implementation}

We developed a prototype of our framework - called HARRSH ${ }^{8}$ - that implements Algorithm 1 as well as all heap automata constructed in the previous sections. The code, the tool and our experiments are available online. ${ }^{9}$

For our experimental results, we first considered common SIDs from the literature, such as singly- and doubly-linked lists, trees, trees with linked-leaves etc. For each of these SIDs, we checked all robustness properties presented throughout this paper, i.e., the existence of points-to assertions (Example 4), the tracking property $\operatorname{TRACK}(B, \Lambda)$ (Section 4.1 ), satisfiability (Section 4.2), establishment (Section 4.3), the reachability property $\operatorname{REACH}(\alpha, R)$ (Section 4.4), garbage-freedom (Section 4.5), and weak acyclicity (Section 4.6). All in all, our implementation of Algorithm 1 takes 300ms to successfully check these properties on all 45 problem instances. Since the SIDs under consideration are typically carefully handcrafted to be robust, the low runtime is to be expected. Moreover, we ran heap automata on benchmarks of the tool CYCLIST [11]. In particular, our results for the satisfiability problem - the only robustness property checked by both tools - were within the same order of magnitude.

Further details are found in Appendix A.2.

\section{Entailment Checking with Heap Automata}

So far, we have constructed heap automata for reasoning about robustness properties, such as satisfiability, establishment and acyclicity. This section demonstrates that our approach can also be applied to discharge entailments for certain fragments of separation logic. Formally, we are concerned with the

Entailment problem (SL-ENTAIL ${ }_{\mathcal{C}}^{\Phi}$ ): Given symbolic heaps $\varphi, \psi \in \mathrm{SH}_{\mathcal{C}}^{\Phi}$, decide whether $\varphi \models_{\Phi} \psi$ holds, i.e., $\forall(s, h) \in$ States . $s, h \models_{\Phi} \varphi$ implies $s, h \models_{\Phi} \psi$.

Note that the symbolic heap fragment of separation logic is not closed under conjunction and negation. Thus, a decision procedure for satisfiability (cf. Theorem 3) does not yield a decision procedure for the entailment problem. It is, however, essential to have a decision procedure for entailment, because this problem underlies the important rule of consequence in Hoare logic [26]. In the words of Brotherston et al. [10], "effective procedures for establishing entailments are at the foundation of automatic verification based on separation logic".

We show how our approach to decide robustness properties, is applicable to discharge entailments for certain fragments of symbolic heaps. This results in an algorithm deciding entailments between so-called determined symbolic heaps for SIDs whose predicates can be characterized by heap automata.

Definition 11. A reduced symbolic heap $\tau$ is determined if all tight models of $\tau$ are isomorphic. ${ }^{10}$ If $\tau$ is also satisfiable then we call $\tau$ well-determined.

\footnotetext{
${ }^{8}$ Heap Automata for Reasoning about Robustness of Symbolic Heaps

9 https://bitbucket.org/jkatelaan/harrsh/

10 A formal definition of model isomorphism is found in Appendix A.1.
} 
Moreover, for some SID $\Phi$, a symbolic heap $\varphi \in \mathrm{SH}^{\Phi}$ is (well-)determined if all of its unfoldings $\tau \in \mathbb{U}_{\Phi}(\varphi)$ are (well-)determined. Consequently, an SID $\Phi$ is (well-)determined if $P \mathbf{x}$ is (well-)determined for each predicate symbol $P$ in $\Phi$.

We present two sufficient conditions for determinedness of symbolic heaps. First, a reduced symbolic heap $\tau$ is determined if all equalities and inequalities between variables are explicit, i.e., $\forall x, y \in \operatorname{Var}(\tau) . x=y \in \Pi^{\tau}$ or $x \neq y \in \Pi^{\tau}$ [30]. Furthermore, a reduced symbolic heap $\tau$ is determined if every variable is definitely allocated or definitely equal to null, i.e., $\forall x \in \operatorname{Var}(\tau) . x \in \operatorname{alloc}(\tau)$ or $x=_{\tau}$ null. These two notions can also be combined: A symbolic heap is determined if every variable $x$ is definitely allocated or definitely equal to null or there is an explicit pure formula $x \sim y$ between $x$ and each other variable $y$.

Example 8. By the previous remark, the SID generating acyclic singly-linked lists from Section 1 is well-determined. Furthermore, although the predicate dll from Example 1 is not determined, the following symbolic heap is welldetermined: $\mathbf{x}_{0}[4] \mapsto$ null $*$ dll $\mathbf{x}_{0}:\left\{\mathbf{x}_{0}[1] \neq \mathbf{x}_{0}[3]\right\}$.

\subsection{Entailment between predicate calls}

We start by considering entailments between predicate calls of well-determined SIDs. By definition, an entailment $\varphi \models_{\Phi} \psi$ holds if for every stack-heap pair $(s, h)$ that satisfies an unfolding of $\varphi$, there exists an unfolding of $\psi$ that is satisfied by $(s, h)$ as well. Our first observation is that, for well-determined unfoldings, two quantifiers can be switched: It suffices for each unfolding $\sigma$ of $\varphi$ to find one unfolding $\tau$ of $\psi$ such that every model of $\sigma$ is also a model of $\tau$.

Lemma 8. Let $\Phi \in \mathrm{SID}$ and $P_{1}, P_{2}$ be predicate symbols with $\operatorname{ar}\left(P_{1}\right)=\operatorname{ar}\left(P_{2}\right)$. Moreover, let $\mathbb{U}_{\Phi}\left(P_{1} \mathbf{x}\right)$ be well-determined. Then

$$
P_{1} \mathbf{x} \models_{\Phi} P_{2} \mathbf{x} \quad \text { iff } \quad \forall \sigma \in \mathbb{U}_{\Phi}\left(P_{1} \mathbf{x}\right) . \exists \tau \in \mathbb{U}_{\Phi}\left(P_{2} \mathbf{x}\right) \cdot \sigma \models_{\emptyset} \tau .
$$

Proof. See Appendix A.21 for a detailed proof.

Note that, even if only well-determined predicate calls are taken into account, it is undecidable in general whether an entailment $P_{1} \mathbf{x}_{0} \models_{\Phi} P_{2} \mathbf{x}_{0}$ holds [1, Theorem 3]. To obtain decidability, we additionally require the set of reduced symbolic heaps entailing a given predicate call to be accepted by a heap automaton.

Definition 12. Let $\Phi \in \operatorname{SID}_{\mathcal{C}}$ and $\varphi \in \mathrm{SH}_{\mathcal{C}}^{\Phi}$. Then

$$
H_{\varphi, \Phi}^{\mathcal{C}} \triangleq\left\{\sigma \in \mathrm{RSH}_{\mathcal{C}} \mid\left\|\mathbf{x}_{0}^{\sigma}\right\|=\left\|\mathbf{x}_{0}^{\varphi}\right\| \text { and } \exists \tau \in \mathbb{U}_{\Phi}(\varphi) . \sigma \models_{\emptyset} \tau\right\}
$$

is the set of all reduced symbolic heaps in $\mathrm{SH}_{\mathcal{C}}$ over the same free variables as $\varphi$ that entail an unfolding of $\varphi$.

Example 9. Let $\varphi=\operatorname{tll} \mathbf{x}_{0}:\left\{\mathbf{x}_{0}[1] \neq \mathbf{x}_{0}[2]\right\}$, where tll is a predicate of SID $\Phi$ introduced in Example 1. Then $H_{\varphi, \Phi}^{\mathrm{FV} \leq 3}$ consists of all reduced symbolic heaps with three free variables representing non-empty trees with linked leaves. In particular, note that these symbolic heaps do not have to be derived using the SID $\Phi$. For instance, they might contain additional pure formulas. 
In particular, $H_{P \mathbf{x}, \Phi}^{\mathcal{C}}$ can be accepted by a heap automaton for common predicates specifying data structures such as lists, trees, and trees with linked leaves. We are now in a position to decide entailments between predicate calls.

Lemma 9. Let $\Phi \in \operatorname{SID}_{\mathcal{C}}$ and $P_{1}, P_{2} \in \operatorname{Pred}(\Phi)$ be predicate symbols having the same arity. Moreover, let $\mathbb{U}_{\Phi}\left(P_{1} \mathbf{x}\right)$ be well-determined and $H_{P_{2} \mathbf{x}, \Phi}^{\mathcal{C}}$ be accepted by a heap automaton over $\mathrm{SH}_{\mathcal{C}}$. Then the entailment $P_{1} \mathbf{x} \models_{\Phi} P_{2} \mathbf{x}$ is decidable.

Proof. Let $\mathfrak{A}_{P_{2} \mathbf{x}}$ be a heap automaton over $\mathrm{SH}_{\mathcal{C}}$ accepting $H_{P_{2} \mathbf{x}, \Phi}^{\mathcal{C}}$. Then

$$
\begin{array}{rlr} 
& P_{1} \mathbf{x} \models_{\Phi} P_{2} \mathbf{x} \\
\Leftrightarrow & \forall \sigma \in \mathbb{U}_{\Phi}\left(P_{1} \mathbf{x}\right) \cdot \exists \tau \in \mathbb{U}_{\Phi}\left(P_{2} \mathbf{x}\right) \cdot \sigma \models_{\emptyset} \tau & (\text { Lemma 8) } \\
\Leftrightarrow & \forall \sigma \in \mathbb{U}_{\Phi}\left(P_{1} \mathbf{x}\right) \cdot \sigma \in H_{P_{2} \mathbf{x}, \Phi}^{\mathcal{C}} & (\text { Definition 12) } \\
\Leftrightarrow & \mathbb{U}_{\Phi}\left(P_{1} \mathbf{x}\right) \subseteq L\left(\mathfrak{A}_{P_{2} \mathbf{x}}\right) . & \left(L\left(\mathfrak{A}_{P_{2} \mathbf{x}}\right)=H_{P_{2} \mathbf{x}, \Phi}^{\mathcal{C}}\right)
\end{array}
$$

where the last inclusion is decidable by Corollary 2 .

\subsection{Entailment between symbolic heaps}

Our next step is to generalize Lemma 9 to arbitrary determined symbolic heaps $\varphi$ instead of single predicate calls. This requires the construction of heap automata $\mathfrak{A}_{\varphi}$ accepting $H_{\varphi, \Phi}^{\mathcal{C}}$. W.l.o.g. we assume SIDs and symbolic heaps to be welldetermined instead of determined only. Otherwise, we apply Theorem 1 with the heap automaton $\mathfrak{A}_{\mathrm{SAT}}$ (cf. Theorem 3) to obtain a well-determined SID. Thus, we restrict our attention to the following set.

Definition 13. The set $\mathrm{SH}_{\langle\alpha\rangle}$ is given by $\langle\alpha\rangle: \mathrm{SH} \rightarrow\{0,1\}$, where $\langle\alpha\rangle(\varphi)=1$ iff $\varphi$ is well-determined and every predicate call of $\varphi$ has $\leq \alpha \in \mathbb{N}$ parameters.

Clearly, $\langle\alpha\rangle$ is decidable, because satisfiability is decidable (cf. Theorem 3) and verifying that a symbolic heap has at most $\alpha$ parameters amounts to a simple syntactic check. Note that, although the number of parameters in predicate calls is bounded by $\alpha$, the number of free variables of a symbolic heap $\varphi \in \mathrm{SH}_{\langle\alpha\rangle}$ is not. We then construct heap automata for well-determined symbolic heaps.

Theorem 10. Let $\alpha \in \mathbb{N}$ and $\Phi \in \operatorname{SID}_{F V \leq \alpha}$ be established. Moreover, for each predicate symbol $P \in \operatorname{Pred}(\Phi)$, let there be a heap automaton over $\mathrm{SH}_{\langle\alpha\rangle}$ accepting $H_{P \mathbf{x}, \Phi}^{\langle\alpha\rangle}$. Then, for every well-determined symbolic heap $\varphi \in \mathrm{SH}^{\Phi}$, there is a heap automaton over $\mathrm{SH}_{\langle\alpha\rangle}$ accepting $H_{\varphi, \Phi}^{\langle\alpha\rangle}$.

Proof. By structural induction on the syntax of symbolic heaps. For each case a suitable heap automaton has to be constructed. See Appendix A.26 fo details.

Remark 3. Brotherston et al. [13] studied the model-checking problem for symbolic heaps, i.e., the question whether $s, h \models_{\Phi} \varphi$ holds for a given stack-heap pair $(s, h)$, an $\operatorname{SID} \Phi$, and a symbolic heap $\varphi \in \mathrm{SH}_{\Phi}$. They showed that this problem 


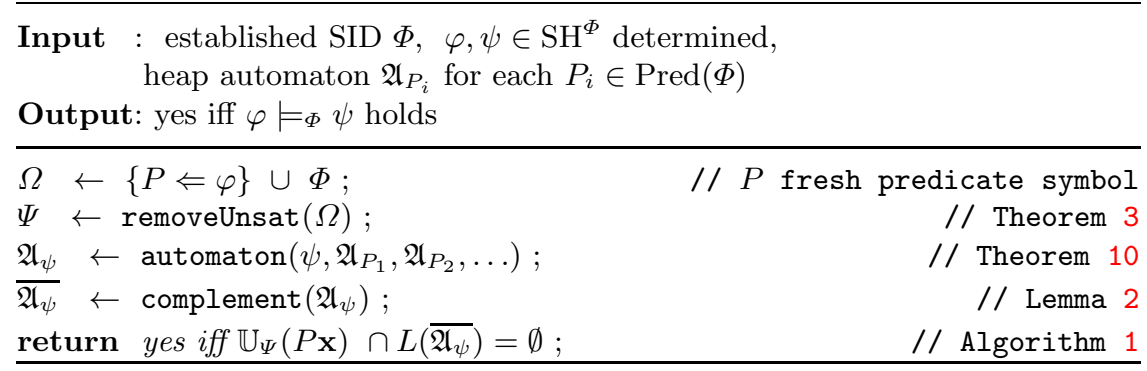

Algorithm 2: Decision procedure for $\varphi \models_{\Phi} \psi$.

is EXPTIME-complete in general and NP-complete if the number of free variables is bounded. We obtain these results for determined symbolic heaps in a natural way: Observe that every stack-heap pair $(s, h)$ is characterized by an established, well-determined, reduced symbolic heap, say $\tau$, that has exactly $(s, h)$ as a tight model up to isomorphism. Then Theorem 10 yields a heap automaton $\mathfrak{A}_{\tau}$ accepting $H_{\tau, \Phi}^{\langle\alpha\rangle}$, where $\alpha$ is the maximal arity of any predicate in $\Phi$. Thus, $s, h \models_{\Phi} \varphi$ iff $L\left(\mathfrak{A}_{\tau}\right) \cap \mathbb{U}_{\Phi}(\varphi) \neq \emptyset$, which is decidable by Corollary 1 . Further, note that the general model-checking problem is within the scope of heap automata.A suitable statespace is the set of all subformulas of the symbolic heap $\tau$.

Coming back to the entailment problem, it remains to put our results together. Algorithm 2 depicts a decision procedure for the entailment problem that, given an entailment $\varphi \models_{\Phi} \psi$, first removes all unsatisfiable unfoldings of $\varphi$, i.e. $\varphi$ becomes well-determined. After that, our previous reasoning techniques for heap automata and SIDs from Section 3 are applied to decide whether $\varphi \models_{\Phi} \psi$ holds. Correctness of Algorithm 2 is formalized in

Theorem 11. Let $\alpha \in \mathbb{N}$ and $\Phi \in \operatorname{SID}_{F V \leq \alpha}$ be established. Moreover, for every $P \in \operatorname{Pred}(\Phi)$, let $H_{P \mathbf{x}, \Phi}^{\langle\alpha\rangle}$ be accepted by a heap automaton over $\mathrm{SH}_{\langle\alpha\rangle}$. Then $\varphi \models_{\Phi} \psi$ is decidable for determined $\varphi, \psi \in \mathrm{SH}^{\Phi}$ with $\mathbf{x}_{0}^{\varphi}=\mathbf{x}_{0}^{\psi}$.

Proof. We define a new SID $\Omega \triangleq \Phi \cup\{P \Leftarrow \varphi\}$, where $P$ is a fresh predicate symbol of arity $\left\|\mathbf{x}_{0}^{\varphi}\right\|$. Clearly, $\varphi \models_{\Phi} \psi$ iff $P \mathbf{x}_{0}^{\varphi} \models_{\Omega} \psi$. Furthermore, since $\varphi$ and $\Phi$ are established, so is $\Omega$. Then applying the Refinement Theorem (Theorem 1) to $\Omega$ and $\mathfrak{A}_{\mathrm{SAT}}$ (cf. Theorem 3), we obtain a well-determined SID $\Psi \in \operatorname{SID}_{\langle\alpha\rangle}$ where none of the remaining unfoldings of $\Omega$ is changed, i.e., for each $P \in \operatorname{Pred}(\Omega)$, we have $\mathbb{U}_{\Psi}(P \mathbf{x}) \subseteq \mathbb{U}_{\Omega}(P \mathbf{x})$. By Theorem 10, the set $H_{\psi, \Phi}^{\langle\alpha\rangle}=H_{\psi, \Psi}^{\langle\alpha\rangle}$ can be accepted by a heap automaton over $\mathrm{SH}_{\langle\alpha\rangle}$. Then, analogously to the proof of Lemma 9 ,

$$
\varphi \models_{\Phi} \psi \text { iff } P \mathbf{x}_{0}^{\varphi} \models_{\Psi} \psi \text { iff } \mathbb{U}_{\Psi}\left(P \mathbf{x}_{0}^{\varphi}\right) \subseteq H_{\psi, \Psi}^{\langle\alpha\rangle},
$$

where the last inclusion is decidable by Corollary 2 . 


\subsection{Complexity}

Algorithm 2 may be fed with arbitrarily large heap automata. For a meaningful complexity analysis, we thus consider heap automata of bounded size only.

Definition 14. An SID $\Phi$ is $\alpha$-bounded if for each $P \in \operatorname{Pred}(\Phi)$ there exists a heap automaton $\mathfrak{A}_{P}$ over $\mathrm{SH}_{\langle\alpha\rangle}$ accepting $H_{P \mathbf{x}, \Phi}^{\langle\alpha\rangle}$ such that $\Delta_{\mathfrak{A}_{P}}$ is decidable in $\mathcal{O}\left(2^{\text {poly }(\|\Phi\|)}\right)$ and $\left\|Q_{\mathfrak{A}_{P}}\right\| \leq 2^{\text {poly }(\alpha)}$.

The bounds from above are natural for a large class of heap automata. In particular, all heap automata constructed in Section 4 stay within these bounds. Then a close analysis of Algorithm 2 for $\alpha$-bounded SIDs yields the following complexity results. A detailed analysis is provided in Appendix A.27.

Theorem 12. SL-ENTAIL $L_{\langle\alpha\rangle}^{\Phi}$ is decidable in 2-EXPTIME for every $\alpha$-bounded SID $\Phi$. If $\alpha \geq 1$ is a constant then SL-ENTAIL ${ }_{\langle\alpha\rangle}^{\Phi}$ is ExPTIME-complete.

Note that lower complexity bounds depend on the SIDs under consideration. Antonopoulos et al. [1, Theorem 6] showed that the entailment problem is already $\Pi_{2}^{P}$-complete ${ }^{11}$ for the base fragment, i.e., $\Phi=\emptyset$. Thus, under common complexity assumptions, the exponential time upper bound derived in Theorem 12 is asymptotically optimal for a deterministic algorithm. Since the entailment problem is already ExPTIME-hard for points-to assertions of arity 3 and SIDs specifying regular sets of trees (cf. [1, Theorem 5] and Appendix A.27.2 for details), exponential time is actually needed for certain SIDs.

\subsection{Expressiveness}

We conclude this section with a brief remark regarding the expressiveness of heap automata. In particular, SIDs specifying common data structures, such as lists, trees, trees with linked leaves and combinations thereof can be encoded by heap automata. ${ }^{12}$ In general, the close relationship between established SIDs and context-free graph languages studied by Dodds [20, Theorem 1] and Courcelle's work on recognizable graph languages [19, Theorems 4.34 and 5.68], suggest that heap automata exist for every set of reduced symbolic heaps that can be specified in monadic second-order logic over graphs [19].

\section{Conclusion}

We developed an algorithmic framework for automatic reasoning about and debugging of the symbolic heap fragment of separation logic. Our approach is centered around a new automaton model, heap automata, that is specifically tailored to symbolic heaps. We show that many common robustness properties as well as certain types of entailments are naturally covered by our frameworkoften with optimal asymptotic complexity. There are several directions for future work including automated learning of heap automata accepting common data structures and applying heap automata to the abduction problem [16].

$\overline{{ }^{11} \Pi_{2}^{P} \text { denotes }}$ the second level of the polynomial hierarchy.

12 Details on the construction of such automata are provided in Appendix A.28. 


\section{References}

1. Antonopoulos, T., Gorogiannis, N., Haase, C., Kanovich, M.I., Ouaknine, J.: Foundations for decision problems in separation logic with general inductive predicates. In: FOSSACS 2014. LNCS, vol. 8412, pp. 411-425. Springer (2014)

2. Berdine, J., Calcagno, C., Cook, B., Distefano, D., O'Hearn, P.W., Wies, T., Yang, H.: Shape analysis for composite data structures. In: CAV 2007. LNCS, vol. 4590, pp. 178-192. Springer (2007)

3. Berdine, J., Calcagno, C., O'Hearn, P.W.: A decidable fragment of separation logic. In: FSTTCS 2004. LNCS, vol. 3328, pp. 97-109 (2004)

4. Berdine, J., Calcagno, C., Ohearn, P.W.: Smallfoot: Modular automatic assertion checking with separation logic. In: International Symposium on Formal Methods for Components and Objects. pp. 115-137. Springer (2005)

5. Berdine, J., Calcagno, C., OHearn, P.W.: Symbolic execution with separation logic. In: APLAS 2005, LNCS, vol. 3780, pp. 52-68. Springer (2005)

6. Berdine, J., Cook, B., Ishtiaq, S.: SLAyer: Memory safety for systems-level code. In: CAV 2011. LNCS, vol. 6806, pp. 178-183. Springer (2011)

7. Bornat, R., Calcagno, C., O'Hearn, P., Parkinson, M.: Permission accounting in separation logic. In: ACM SIGPLAN Notices. vol. 40, pp. 259-270. ACM (2005)

8. Botincan, M., Distefano, D., Dodds, M., Grigore, R., Naudziuniene, D., Parkinson, M.J.: corestar: The core of jstar. BOOGIE 2011, 65-77 (2011)

9. Brookes, S.: A semantics for concurrent separation logic. Theoretical Computer Science 375(1), 227-270 (2007)

10. Brotherston, J., Distefano, D., Petersen, R.L.: Automated cyclic entailment proofs in separation logic. In: CADE-23. LNAI, vol. 6803, pp. 131-146. Springer (2011)

11. Brotherston, J., Fuhs, C., Pérez, J.A.N., Gorogiannis, N.: A decision procedure for satisfiability in separation logic with inductive predicates. In: CSL-LICS 2014. pp. 25:1-25:10. ACM (2014)

12. Brotherston, J., Gorogiannis, N.: Cyclic abduction of inductively defined safety and termination preconditions. In: SAS 2014. LNCS, vol. 8723, pp. 68-84. Springer (2014)

13. Brotherston, J., Gorogiannis, N., Kanovich, M.I., Rowe, R.: Model checking for symbolic-heap separation logic with inductive predicates. In: POPL 2016. pp. 8496. ACM (2016)

14. Brotherston, J., Gorogiannis, N., Petersen, R.L.: A generic cyclic theorem prover. In: APLAS 2012. pp. 350-367. Springer (2012)

15. Calcagno, C., Distefano, D.: Infer: An automatic program verifier for memory safety of C programs. In: NFM 2011. LNCS, vol. 6617, pp. 459-465. Springer (2011)

16. Calcagno, C., Distefano, D., O'Hearn, P., Yang, H.: Compositional shape analysis by means of bi-abduction. In: POPL 2009. pp. 289-300. ACM (2009)

17. Chin, W., David, C., Nguyen, H.H., Qin, S.: Automated verification of shape, size and bag properties via user-defined predicates in separation logic. Sci. Comput. Program. 77(9), 1006-1036 (2012)

18. Comon, H., Dauchet, M., Gilleron, R., Löding, C., Jacquemard, F., Lugiez, D., Tison, S., Tommasi, M.: Tree automata techniques and applications (2007), available at http://www.grappa.univ-lille3.fr/tata

19. Courcelle, B., Engelfriet, J.: Graph structure and monadic second-order logic: a language-theoretic approach, vol. 138. Cambridge University Press (2012)

20. Dodds, M.: From separation logic to hyperedge replacement and back. In: ICGT 2008. pp. 484-486. Springer (2008) 
21. Dudka, K., Peringer, P., Vojnar, T.: Predator: A practical tool for checking manipulation of dynamic data structures using separation logic. In: CAV 2011. LNCS, vol. 6806, pp. 372-378. Springer (2011)

22. Enea, C., Lengál, O., Sighireanu, M., Vojnar, T.: Compositional entailment checking for a fragment of separation logic. In: APLAS 2014. LNCS, vol. 8837, pp. 314-333. Springer (2014)

23. Gotsman, A., Berdine, J., Cook, B., Sagiv, M.: Thread-modular shape analysis. In: PLDI 2007. pp. 266-277. ACM (2007)

24. Habermehl, P., Holík, L., Rogalewicz, A., Šimáček, J., Vojnar, T.: Forest automata for verification of heap manipulation. In: CAV 2011. LNCS, vol. 6806, pp. 424-440. Springer (2011)

25. Habermehl, P., Holík, L., Rogalewicz, A., Šimáček, J., Vojnar, T.: Forest automata for verification of heap manipulation. Formal Methods in System Design 41(1), 83$106(2012)$

26. Hoare, C.A.R.: An axiomatic basis for computer programming. Communications of the ACM 12(10), 576-580 (1969)

27. Iosif, R., Rogalewicz, A., Simacek, J.: The tree width of separation logic with recursive definitions. In: CADE-24. LNCS, vol. 7898, pp. 21-38. Springer (2013)

28. Iosif, R., Rogalewicz, A., Vojnar, T.: Deciding entailments in inductive separation logic with tree automata. In: ATVA 2014. LNCS, vol. 8837, pp. 201-218. Springer (2014)

29. Jacobs, B., Smans, J., Philippaerts, P., Vogels, F., Penninckx, W., Piessens, F.: Verifast: A powerful, sound, predictable, fast verifier for $\mathrm{C}$ and Java. In: NFM 2011. LNCS, vol. 6617, pp. 41-55. Springer (2011)

30. Jansen, C., Katelaan, J., Matheja, C., Noll, T., Zuleger, F.: Unified Reasoning about Robustness Properties of Symbolic-Heap Separation Logic. ArXiv e-prints (Oct 2016)

31. Le, Q.L., Gherghina, C., Qin, S., Chin, W.N.: Shape analysis via second-order bi-abduction. In: CAV 2014. LNCS, vol. 8559, pp. 52-68. Springer (2014)

32. Magill, S., Tsai, M.H., Lee, P., Tsay, Y.K.: Thor: A tool for reasoning about shape and arithmetic. In: CAV 2008. LNCS, vol. 5123, pp. 428-432. Springer (2008)

33. Navarro Pérez, J., Rybalchenko, A.: Separation logic modulo theories. In: APLAS 2013, LNCS, vol. 8301, pp. 90-106. Springer (2013)

34. Nerode, A.: Linear automaton transformations. Proceedings of the American Mathematical Society 9(4), 541-544 (1958)

35. Nguyen, H.H., Kuncak, V., Chin, W.N.: Runtime checking for separation logic. In: VMCAI 2008. LNCS, vol. 4905, pp. 203-217. Springer (2008)

36. O'Hearn, P.W.: Resources, concurrency, and local reasoning. Theor. Comput. Sci. 375(1-3), 271-307 (2007)

37. OHearn, P., Reynolds, J., Yang, H.: Local reasoning about programs that alter data structures. In: International Workshop on Computer Science Logic. pp. 1-19. Springer (2001)

38. Piskac, R., Wies, T., Zufferey, D.: Automating separation logic with trees and data. In: CAV 2014. LNCS, vol. 8559, pp. 711-728. Springer (2014)

39. Qiu, X., Garg, P., Ştefănescu, A., Madhusudan, P.: Natural proofs for structure, data, and separation. In: PLDI 2013. pp. 231-242. ACM (2013)

40. Reynolds, J.C.: Separation logic: A logic for shared mutable data structures. In: LICS 2002. pp. 55-74. IEEE (2002)

41. Zanardini, D., Genaim, S.: Inference of field-sensitive reachability and cyclicity. ACM Trans. Comput. Log. 15(4), 33:1-33:41 (2014) 


\section{A.1 Supplementary Material}

Definition 15 (Isomorphic states). Two states $\left(s_{1}, h_{1}\right),\left(s_{2}, h_{2}\right)$ are isomorphic if and only if there exist bijective functions $f: \operatorname{dom}\left(s_{1}\right) \rightarrow \operatorname{dom}\left(s_{2}\right) g$ : $\operatorname{dom}\left(h_{1}\right) \rightarrow \operatorname{dom}\left(h_{2}\right)$ such that for all $\ell \in \operatorname{dom}\left(h_{1}\right)$, we have $g\left(h_{1}(\ell)\right)=h_{2}(g(\ell))$, where $g$ is lifted to tuples by componentwise application.

\section{A.2 Implemenation and Experimental Results}

Overall, the implementation of our tool HARRSH consists of about 1500 lines of Scala code, not counting test classes, comments and blank lines. Since our tool is - to our best knowledge - the first one to systematically reason about robustness properties, we cannot compare the results of our tool against other implementations.

A notable exception is CyCLIST [11], which is capable of proving satisfiability of SIDs. We evaluated our tool against the large collection of benchmarks that is distributed with CyCLIST. In particular, this collection includes the following sets:

1. A set of handwritten standard predicates from the separation logic literature.

2. 45945 problem instances that have been automatically generated by the inference tool CABER [12].

3. A set of particularly hard problem instances that are derived from the SIDs used to prove lower complexity bounds for satisfiability. These benchmarks have been used to test the scalability of CYCLIST.

Experiments were performed on an Intel Core i5-3317U at $1.70 \mathrm{GHz}$ with 4GB of RAM.

For the standard predicates in the first set, our implementation runs in total approximately $300 \mathrm{~ms}$ to check all robustness properties on all standard predicates, i.e., a total of 45 problem instances. As already reported in the paper, this low analysis time is not a surprise, because the standard data structure predicates are generally very well-behaved.

To evaluate the performance of HARRSH on a realistic set of benchmarks, we ran both HARRSH and CYCLIST on all 45945 benchmarks generated by CABER. For CyCLIST, we only checked satisfiability - the only of the robustness properties supported by CyClist; for HARRsh, we checked all robustness properties introduced in Section 4.

Both tools were capable of proving (un)satisfiability of all of these problem instances within a set timeout of 30 seconds. All in all, the accumulated analysis time of HARRSH for these instances was $12460 \mathrm{~ms}$, while CYCLIST required $44856 \mathrm{~ms} .{ }^{13}$ For all other properties, HARRSH also achieved accumulated analysis time below 20 seconds; see Table 1 . These numbers demonstrate the applicability of our tool to problem instances that occur in practice.

\footnotetext{
${ }^{13}$ For both tools we added up the analysis times of individual tasks, reported with millisecond precision. Consequently, we expect that rounding errors influence the accumulated time to a similar degree for both tools.
} 


\begin{tabular}{l|r} 
Robustness Property & Analysis Time (ms) \\
\hline No points-to assertions (Example 4) & 7230 \\
Tracking property (Section 4.1) & 11459 \\
Satisfiability (Section 4.2) & 12460 \\
Complement of Satisfiability (Section 4.2) & 11980 \\
Establishment (Section 4.3) & 18055 \\
Complement of Establishment (Section 4.3) & 17272 \\
Reachability (Section 4.4) & 14897 \\
Garbage-Freedom (Section 4.5) & 18192 \\
Weak Acyclicity (Section 4.6) & 18505
\end{tabular}

Table 1. Total analysis time for robustness properties presented throughout the paper on the second set of benchmarks, i.e., 45945 automatically inferred SIDs.

Moreover, we ran HARRSH and CYCLIST to check satisfiability of the third set and additional handwritten benchmarks distributed with CYCLIST. For both tools, we chose a timeout of 5 minutes. The measured analysis times for this set are shown in Table 2.

Notably, the tools yield different results for the SID contained in the file inconsistent-ls-of-ls.def. While HARRSH states that this SID is satisfiable, CyClist states that it is not. Despite the benchmark's name, however, the underlying SID

$$
\begin{aligned}
& P \Leftarrow x=\text { null } \\
& P \Leftarrow Q(x x):\{x \neq \text { null }\} \\
& Q \Leftarrow \exists c, d . x \mapsto(d, c) * P(d):\{y=\text { null }, x \neq \text { null }\} \\
& Q \Leftarrow \exists c, d . x \mapsto(d, c):\{y \neq \text { null }\}
\end{aligned}
$$

is satisfiable: Clearly $x=$ null is a satisfiable unfolding of $P x$. Using this unfolding to replace the predicate call $P(d)$ in the third rule, we also obtain a a satisfiable unfolding of $Q(x, y)$ :

$$
\exists c, d . x \mapsto(d, c):\{d=\text { null, } y=\text { null, } x \neq \text { null }\}
$$

\section{A.3 Proof of Lemma 1}

By induction on the height $k$ of unfolding trees of $\varphi$.

I.B. If $k=0$ then $\left\|\Gamma^{\varphi}\right\|=0$, i.e., $\varphi$ contains no predicate calls. Thus $\llbracket t \rrbracket=\varphi$. Then, for each $(s, h) \in$ States, we have

$$
\begin{aligned}
& s, h \models_{\Phi} \varphi \\
\Leftrightarrow & {\left[\left\|\Gamma^{\varphi}\right\|=0\right] } \\
& s, h \models_{\emptyset} \varphi
\end{aligned}
$$




\begin{tabular}{l|r|r} 
Benchmark & HARRSH & CYCLIST \\
\hline inconsistent-ls-of-ls.defs & 1 & 4 (not correct) \\
succ-rec01.defs & 3 & 0 \\
succ-rec02.defs & 10 & 8 \\
succ-rec03.defs & 24 & 12 \\
succ-rec04.defs & 106 & 20 \\
succ-rec05.defs & 496 & 128 \\
succ-rec06.defs & 2175 & 792 \\
succ-rec07.defs & 9692 & 4900 \\
succ-rec08.defs & 39408 & 31144 \\
succ-rec09.defs & 169129 & 164464 \\
succ-rec10.defs & TO & TO \\
succ-circuit01.defs & 80 & 4 \\
succ-circuit02.defs & 142 & 8 \\
succ-circuit03.defs & 699 & 48 \\
succ-circuit04.defs & 4059 & 832 \\
succ-circuit05.defs & 75110 & 28800 \\
succ-circuit06.defs & TO & TO
\end{tabular}

Table 2. Comparison of HARRSH and CyClist for hard instances of the satisfiability problem. Provided times are in milliseconds. Timeouts (TO) are set to 5 minutes.

$$
\begin{aligned}
\Leftrightarrow \quad & {\left[\mathbb{U}_{\Phi}(\varphi)=\{\varphi\}\right] } \\
& \exists \tau \in \mathbb{U}_{\Phi}(\varphi) . s, h \models_{\emptyset} \tau .
\end{aligned}
$$

I.H. Assume for an arbitrary, but fixed, natural number $k$ that for each $\Phi \in$ SID, $\varphi \in \mathrm{SH}^{\Phi}$, where each $t \in \mathbb{T}_{\Phi}(\varphi)$ is of height at most $k$, it holds for each $(s, h) \in$ States that

$$
s, h \models_{\Phi} \varphi \quad \text { iff } \quad \exists \tau \in \mathbb{U}_{\Phi}(\varphi) . s, h \models_{\emptyset} \tau .
$$

I.S. Let $\Phi \in \mathrm{SID}$ and $\varphi \in \mathrm{SH}^{\Phi}$ such that each $t \in \mathbb{T}_{\Phi}(\varphi)$ is of height at most $k+1$. We proceed by structural induction on the syntax of $\varphi$. For $\varphi=\operatorname{emp}$, $\varphi=x \mapsto(\mathbf{a}), \varphi=(a=b), \varphi=(a \neq b)$, the height of all unfolding trees is $0<k+1$, i.e., there is nothing to show. If $\varphi=P \mathbf{a}$ then $\llbracket t \rrbracket=\left.\llbracket t\right|_{1} \rrbracket$ holds for each unfolding of $\varphi$. Since $t$ is of height at most $k+1,\left.t\right|_{1}$ is of height at most $k$. By I.H. we obtain for each $(s, h) \in$ States that

$$
s, h \models_{\Phi} P \mathbf{a} \quad \text { iff } \quad \exists \tau \in \mathbb{U}_{\Phi}(P \mathbf{a}) . s, h \models_{\emptyset} \tau .
$$

If $\varphi=\varphi_{1} * \varphi_{2}$, we have for each $(s, h) \in$ States:

$$
s, h \models_{\Phi} \varphi_{1} * \varphi_{2}
$$

$\Leftrightarrow \quad$ Semantics of $*]$ $\exists h_{1}, h_{2} . h=h_{1} \uplus h_{2}$ and $s, h_{1} \models_{\Phi} \varphi_{1}$ and $s, h_{2} \models_{\Phi} \varphi_{2}$

$\Leftrightarrow$ [I.H. on $\left.\varphi_{1}\right]$ 


$$
\begin{aligned}
& \exists h_{1}, h_{2} \cdot h=h_{1} \uplus h_{2} \\
& \quad \text { and } \exists \tau_{1} \in \mathbb{U}_{\Phi}\left(\varphi_{1}\right) . s, h_{1} \models_{\Phi} \tau_{1} \\
& \quad \text { and } s, h_{2} \models_{\Phi} \varphi_{2} \\
\Leftrightarrow & {\left[\text { I.H. on } \varphi_{2}\right] } \\
& \exists h_{1}, h_{2} \cdot h=h_{1} \uplus h_{2} \\
& \quad \text { and } \exists \tau_{1} \in \mathbb{U}_{\Phi}\left(\varphi_{1}\right) . s, h_{1} \models_{\emptyset} \tau_{1} \\
& \quad \text { and } \exists \tau_{2} \in \mathbb{U}_{\Phi}\left(\varphi_{2}\right) . s, h_{2} \models_{\emptyset} \tau_{2} \\
\Leftrightarrow & {\left[\mathbb{U}_{\Phi}\left(\varphi_{1} * \varphi_{2}\right)=\left\{\left(\varphi_{1} * \varphi_{2}\right)\left[\Gamma^{\varphi_{1}} / \tau_{1}, \Gamma^{\varphi_{2}} / \tau_{2}\right] \mid \tau_{1} \in \mathbb{U}_{\Phi}\left(\varphi_{1}\right),\right.\right.} \\
& \exists \tau \in \mathbb{U}_{\Phi}\left(\varphi_{1} * \varphi_{2}\right) . s, h \models_{\emptyset} \tau
\end{aligned}
$$

Finally, we consider the case $\varphi=\exists \mathbf{z} . \Sigma * \Gamma: \Pi$. The crux of the proof relies on the observation that for each $t \in \mathbb{T}_{\Phi}(\varphi)$ and each $t^{\prime} \in \mathbb{T}_{\Phi}(\Sigma * \Gamma)$, we have $t(\varepsilon)=\exists \mathbf{z} \cdot t^{\prime}(\varepsilon): \Pi$. Thus

$$
\mathbb{U}_{\Phi}(\varphi)=\left\{\exists \mathbf{z} \cdot \tau^{\prime}: \Pi \mid \tau^{\prime} \in \mathbb{U}_{\Phi}(\Sigma * \Gamma)\right\} .
$$

Then we have for each $(s, h) \in$ States:

$$
\begin{aligned}
& s, h \models_{\Phi} \exists \mathbf{z} . \Sigma * \Gamma: \Pi \\
\Leftrightarrow & {[\text { SL semantics] }} \\
& \exists \mathbf{v} \in \operatorname{Val}^{\|\mathbf{z}\|} . s[\mathbf{z} \mapsto \mathbf{v}], h \models_{\Phi} \Sigma * \Gamma \\
& \quad \text { and } \forall \pi \in \Pi . s[\mathbf{z} \mapsto \mathbf{v}], h \models_{\Phi} \pi \\
\Leftrightarrow & {[\text { I.H. on } \Sigma * \Gamma] } \\
& \exists \mathbf{v} \in \operatorname{Val}^{\|\mathbf{z}\|} \cdot \exists \tau^{\prime} \in \mathbb{U}_{\Phi}(\Sigma * \Gamma) . s[\mathbf{z} \mapsto \mathbf{v}], h \models_{\emptyset} \tau^{\prime} \\
& \quad \text { and } \forall \pi \in \Pi . s[\mathbf{z} \mapsto \mathbf{v}], h \models_{\Phi} \pi \\
\Leftrightarrow & {[\exists x \exists y \equiv \exists y \exists x] } \\
& \exists \tau^{\prime} \in \mathbb{U}_{\Phi}(\Sigma * \Gamma) . \exists \mathbf{v} \in \operatorname{Val} l^{\|\mathbf{z}\|} . s[\mathbf{z} \mapsto \mathbf{v}], h \models_{\emptyset} \tau^{\prime} \\
& \text { and } \forall \pi \in \Pi . s[\mathbf{z} \mapsto \mathbf{v}], h \models_{\Phi} \pi \\
\Leftrightarrow & {[\text { apply }(\dagger)] } \\
& \exists \tau \in \mathbb{U}_{\Phi}(\varphi) . s, h \models_{\emptyset} \tau .
\end{aligned}
$$

\section{A.4 Coincidence Lemma for Symbolic Heaps}

Lemma 10. Let $\Phi \in \mathrm{SID}$ and $\varphi \in \mathrm{SH}^{\Phi}$. Moreover, let $(s, h) \in$ States. Then $s, h \models_{\Phi} \varphi$ iff $\left(\left.s\right|_{\mathbf{x}_{0}^{\varphi}} ^{\varphi}\right), h \models_{\Phi} \varphi$, where $\left.s\right|_{\mathbf{x}_{0}^{\varphi}}$ denotes the restriction of the domain of $s$ to the free variables of $\varphi$.

Proof. By structural induction on the syntax of symbolic heaps $\varphi$. 
$\varphi=\mathrm{emp}$

$$
\begin{aligned}
& s, h \models_{\Phi} \text { emp } \\
\Leftrightarrow \quad & {[\text { SL semantics }] } \\
& \operatorname{dom}(h)=\emptyset \\
\Leftrightarrow \quad & {\left[\mathbf{x}_{0}^{\text {emp }}=\emptyset\right] } \\
& \left(\left.s\right|_{\mathbf{x}_{0}^{\text {emp }}}\right), h \models_{\Phi} \text { emp. }
\end{aligned}
$$

$\varphi=x \mapsto(\mathbf{a})$

$$
s, h \models_{\Phi} x \mapsto(\mathbf{a})
$$

$\Leftrightarrow$ [SL semantics]

$\operatorname{dom}(h)=\{s(x)\}$ and $h(s(x))=s(\mathbf{a})$

$$
\begin{aligned}
\Leftrightarrow \quad & {\left[\mathbf{x}_{0}^{x \mapsto(\mathbf{a})}=\{x\} \cup \mathbf{a}\right] } \\
& \left(\left.s\right|_{\mathbf{x}_{0}^{x \mapsto(\mathbf{a})}}\right), h \models_{\Phi} x \mapsto(\mathbf{a}) .
\end{aligned}
$$

$\varphi=(a \sim b), \quad \sim \in\{=, \neq\}$

$$
\begin{aligned}
& s, h \models_{\Phi} a \sim b \\
\Leftrightarrow \quad & {[\text { SL semantics }] } \\
& s(a) \sim s(b) \\
\Leftrightarrow \quad & {\left[\mathbf{x}_{0}^{a \sim b}=\{a, b\}\right] } \\
& \left(s \uparrow_{\mathbf{x}_{0}^{a \sim b}}\right), h \models_{\Phi} a \sim b .
\end{aligned}
$$

$\varphi=\sigma * \tau$

$$
\begin{aligned}
& s, h \models_{\Phi} \sigma * \tau \\
& \Leftrightarrow \quad \text { SL semantics }] \\
& \exists h_{1}, h_{2} \cdot h=h_{1} \uplus h_{2} \text { and } s, h_{1} \models_{\Phi} \sigma \text { and } s, h_{2} \models_{\Phi} \tau \\
& \Leftrightarrow \text { [I.H.] } \\
& \exists h_{1}, h_{2} \cdot h=h_{1} \uplus h_{2} \\
& \text { and }\left(s \uparrow_{\mathbf{x}_{0}^{\sigma}}, h_{1}\right) \models_{\Phi} \sigma \text { and }\left(s \uparrow_{\mathbf{x}_{0}^{\tau}}, h_{2}\right) \models_{\Phi} \tau \\
& \Leftrightarrow\left[\mathbf{x}_{0}^{\tau}, \mathbf{x}_{0}^{\sigma} \subseteq \operatorname{dom}(s), \text { I.H. }\right] \\
& \exists h_{1}, h_{2} \cdot h=h_{1} \uplus h_{2} \\
& \text { and }\left(s \lceil \mathbf { x } _ { 0 } ^ { \sigma } \cup \mathbf { x } _ { 0 } ^ { \tau } , h _ { 1 } ) \models _ { \Phi } \sigma \text { and } \left(s\left\lceil\mathbf{x}_{0}^{\sigma} \cup \mathbf{x}_{0}^{\tau}, h_{2}\right) \models_{\Phi} \tau\right.\right. \\
& \Leftrightarrow\left[\mathbf{x}_{0}^{\sigma * \tau}=\mathbf{x}_{0}^{\sigma} \cup \mathbf{x}_{0}^{\tau}, \text { SL semantics }\right] \\
& \left(s \uparrow \mathbf{x}_{0}^{\sigma * \tau}\right), h \models_{\Phi} \sigma * \tau \text {. }
\end{aligned}
$$


$\varphi=P \mathbf{a}$

$$
\begin{aligned}
& s, h \models_{\Phi} P \mathbf{a} \\
\Leftrightarrow & {[\text { SL semantics }] } \\
& \exists \tau \in \mathbb{U}_{\Phi}(P \mathbf{a}) s, h \models_{\emptyset} \tau \\
\Leftrightarrow & {[\text { I.H. }] } \\
& \exists \tau \in \mathbb{U}_{\Phi}(P \mathbf{a})\left(s\left\lceil_{\mathbf{x}_{0}^{\tau}}^{\tau}\right), h \models_{\Phi} \tau\right. \\
\Leftrightarrow & {\left[\mathbf{x}_{0}^{P \mathbf{a}}=\mathbf{x}_{0}^{\tau}\right] } \\
& \left(s \uparrow_{\mathbf{x}_{0}^{P \mathbf{a}}}\right), h \models_{\Phi} P \mathbf{a} .
\end{aligned}
$$

$\varphi=\exists \mathbf{z} . \Sigma * \Gamma: \Pi$

$$
\begin{aligned}
& s, h \models_{\Phi} \exists \mathbf{z} . \Sigma * \Gamma: \Pi \\
& \Leftrightarrow \text { [SL semantics] } \\
& \exists \mathbf{v} \in \operatorname{Val}^{\|\mathbf{z}\|} . s[\mathbf{z} \mapsto \mathbf{v}], h \models_{\Phi} \Sigma * \Gamma \\
& \text { and } \forall \pi \in \Pi . s[\mathbf{z} \mapsto \mathbf{v}], h \models_{\Phi} \pi \\
& \Leftrightarrow[\text { I.H. on } \Sigma * \Gamma \text { and } \pi] \\
& \exists \mathbf{v} \in \operatorname{Val}^{\|\mathbf{z}\|} .\left(\left.s[\mathbf{z} \mapsto \mathbf{v}]\right|_{\left.\mathbf{x}_{0}^{\Sigma * \Gamma}\right)}\right), h \models_{\Phi} \Sigma * \Gamma \\
& \text { and } \forall \pi \in \Pi \text {. }\left(s[\mathbf{z} \mapsto \mathbf{v}]\left\lceil\mathbf{x}_{0}^{\pi}\right), h \models_{\Phi} \pi\right. \\
& \Leftrightarrow \quad\left[\operatorname{dom}\left(s[\mathbf{z} \mapsto \mathbf{v}] \Gamma_{\mathbf{x}_{0}^{\pi}}\right) \subseteq \operatorname{dom}\left(\left(s \uparrow_{\mathbf{x}_{0}^{\pi}}\right)[\mathbf{z} \mapsto \mathbf{v}]\right)\right] \\
& \exists \mathbf{v} \in \operatorname{Val}^{\|\mathbf{z}\|} .\left(s \uparrow_{\mathbf{x}_{0}^{\Sigma * \Gamma}}[\mathbf{z} \mapsto \mathbf{v}]\right), h \models_{\Phi} \Sigma * \Gamma \\
& \text { and } \forall \pi \in \Pi .\left(s\left\lceil_{\mathbf{x}_{0}^{\pi}}[\mathbf{z} \mapsto \mathbf{v}]\right), h \models_{\Phi} \pi\right. \\
& \Leftrightarrow\left[\mathbf{x}_{0}^{\varphi}=\left(\mathbf{x}_{0}^{\Sigma * \Gamma} \cup \bigcup_{\pi \in \Pi} \mathbf{x}_{0}^{\pi}\right) \backslash \mathbf{z}\right] \\
& \exists \mathbf{v} \in \operatorname{Val}^{\|\mathbf{z}\|} .\left(s\left\lceil_{\mathbf{x}_{0}^{\varphi}}[\mathbf{z} \mapsto \mathbf{v}]\right), h \models_{\Phi} \Sigma * \Gamma\right. \\
& \text { and } \forall \pi \in \Pi .\left(s\left\lceil_{\mathbf{x}_{0}^{\varphi}}[\mathbf{z} \mapsto \mathbf{v}]\right), h \models_{\Phi} \pi\right. \\
& \Leftrightarrow \text { [SL semantics }] \\
& \left(s \uparrow_{\mathbf{x}_{0}^{\varphi}}^{\varphi}\right), h \models_{\Phi} \exists \mathbf{z} . \Sigma * \Gamma: \Pi .
\end{aligned}
$$

\section{A.5 The emptiness problem for sets of unfolding trees}

The set of unfolding trees of a given symbolic heap $\varphi$ with predicates taken from an SID $\Phi$ can be accepted by a bottom-up tree automaton $\mathfrak{A}$. Then, the set of unfolding trees of $\varphi$ is empty if and only if $\mathfrak{A}$ accepts the empty language (of trees). Since the emptiness problem of tree automata is PTIME-complete (cf. [18, Theorem 1.7.4]), so is the question whether the set of unfolding trees of a given symbolic heap is empty. 
Formally, let $\Phi \in \mathrm{SID}$ and $\varphi \in \mathrm{SH}^{\Phi}$. Then the set of unfolding trees of $\varphi$ w.r.t. $\Phi$ is the set of all trees accepted by the tree automaton $\mathfrak{A}=(Q, A, \Delta, F)$, where

$$
\begin{aligned}
Q & =\{P \mid P \Leftarrow \psi \in \Phi\} \uplus\{S\}, \quad F=\{S\}, \\
A & =\{\psi \mid P \Leftarrow \psi \in \Phi\} \cup\{\varphi\}, \\
P_{1} \ldots P_{m} \stackrel{\psi}{\rightarrow} P_{0} & \text { iff } P_{0} \Leftarrow \psi \in \Phi \text { or } \psi=\varphi \text { and } P_{0}=S,
\end{aligned}
$$

where $\Gamma^{\psi}=P_{1} \mathbf{x}_{1} * \ldots * P_{m} \mathbf{x}_{m}$.

\section{A.6 Proof of Theorem 2}

Theorem 2. Let $\mathfrak{A}$ and $\mathfrak{B}$ be heap automata over $\mathrm{SH}_{\mathcal{C}}$. Then there exists a heap automata $\mathfrak{C}_{1}, \mathfrak{C}_{2}, \mathfrak{C}_{3}$ over $\mathrm{SH}_{\mathcal{C}}$ with $L\left(\mathfrak{C}_{1}\right)=L(\mathfrak{A}) \cup L(\mathfrak{B}), L\left(\mathfrak{C}_{2}\right)=L(\mathfrak{A}) \cap L(\mathfrak{B})$, and $L\left(\mathfrak{C}_{3}\right)=\mathrm{RSH}_{\mathcal{C}} \backslash L(\mathfrak{A})$, respectively.

Proof. We split the proof across three lemmas that are proven subsequently. Closure under union and intersection are shown in Lemma 11 and Lemma 12, respectively. Finally, closure under complement with respect to $\mathrm{RSH}_{\mathcal{C}}$ is proven in Lemma 13.

Lemma 11. Let $\mathfrak{A}, \mathfrak{B}$ be heap automata over $\mathrm{SH}_{\mathcal{C}}$. Then there exists a heap automaton $\mathfrak{C}$ over $\mathrm{SH}_{\mathcal{C}}$ such that $L(\mathfrak{C})=L(\mathfrak{A}) \cup L(\mathfrak{B})$.

Proof. We construct a heap automaton $\mathfrak{C}=\left(Q, \mathrm{SH}_{\mathcal{C}}, \Delta, F\right)$ as follows:

$$
\begin{gathered}
Q \triangleq\left(Q_{\mathfrak{A}} \cup\{\perp\}\right) \times\left(Q_{\mathfrak{B}} \cup\{\perp\}\right) \\
F \triangleq F_{\mathfrak{A}} \times\{\perp\} \cup\{\perp\} \times F_{\mathfrak{B}} \\
\Delta:\left(p_{1}, q_{1}\right) \ldots\left(p_{m}, q_{m}\right) \stackrel{\varphi}{\rightarrow}_{\mathfrak{C}}\left(p_{0}, q_{0}\right) \Leftrightarrow \\
p_{1} \ldots p_{m} \stackrel{\varphi}{\rightarrow}_{\mathfrak{A}} p_{0} \text { and } p_{2}=\perp \text { or } \\
q_{1} \ldots q_{m} \stackrel{\varphi}{\rightarrow}_{\mathfrak{B}} q_{0} \text { and } p_{1}=\perp,
\end{gathered}
$$

where $\varphi \in \mathrm{SH}_{\mathcal{C}}$ with $\left\|\Gamma^{\varphi}\right\|=m$. Moreover, $\perp$ is assumed to be a fresh state.

Clearly, $\Delta$ is decidable, because $\Delta_{\mathfrak{A}}$ and $\Delta_{\mathfrak{B}}$ are. Assuming $\mathfrak{C}$ satisfies the compositionality property, we first show that the language of $\mathfrak{C}$ in fact accepts $L(\mathfrak{A}) \cup L(\mathfrak{B}):$

$$
\begin{aligned}
& \tau \in L(\mathfrak{A}) \cup L(\mathfrak{B}) \\
\Leftrightarrow & {[\text { Definition of } \cup] } \\
& \tau \in L(\mathfrak{A}) \text { or } \tau \in L(\mathfrak{B}) \\
\Leftrightarrow & {[\text { Definition of } L(\mathfrak{A}), L(\mathfrak{B})] } \\
& \exists p \in F_{\mathfrak{A}} \cdot \varepsilon \stackrel{\tau}{\rightarrow}_{\mathfrak{A}} p \text { or } \exists q \in F_{\mathfrak{B}} \cdot \varepsilon \stackrel{\tau}{\rightarrow}_{\mathfrak{B}} q \\
\Leftrightarrow & {[\text { construction of } \mathfrak{C}] }
\end{aligned}
$$




$$
\begin{aligned}
& \exists p \in F_{\mathfrak{A}} \cdot \varepsilon \stackrel{\tau}{\rightarrow}_{\mathfrak{C}}(p, \perp) \text { or } \exists q \in F_{\mathfrak{B}} \cdot \varepsilon \stackrel{\tau}{\rightarrow}_{\mathfrak{C}}(\perp, q) \\
\Leftrightarrow & {[\text { Definition of } F] } \\
& \exists(p, q) \in F \cdot \varepsilon \stackrel{\tau}{\rightarrow}_{\mathfrak{C}}(p, q) .
\end{aligned}
$$

It remains to show the compositionality property, i.e., for each $p \in Q, \varphi \in \mathrm{SH}_{\mathcal{C}}$ with $\left\|\Gamma^{\varphi}\right\|=m$ and $\tau_{1}, \ldots, \tau_{m} \in \mathrm{RSH}_{\mathcal{C}}$ with $\sigma=\varphi\left[P_{1} / \tau_{1}, \ldots, P_{m} / \tau_{m}\right]$, we have

$$
\varepsilon \stackrel{\sigma}{\rightarrow} \mathfrak{C} p \Leftrightarrow \exists \mathbf{q} \in Q^{m} \cdot \mathbf{q} \stackrel{\varphi}{\rightarrow} \mathfrak{C} p \text { and } \forall 1 \leq i \leq m . \varepsilon \stackrel{\tau_{i}}{\longrightarrow} \mathfrak{C} \mathbf{q}[i] .
$$

In the following proof, we write $p_{1}, p_{2}$ to denote the first and second component of $p \in Q$, respectively.

$$
\begin{aligned}
& \varepsilon \stackrel{\sigma}{\rightarrow} \mathfrak{C} p \\
\Leftrightarrow & {[\text { construction of } \mathfrak{C}] } \\
& p_{2}=\perp \text { and } \varepsilon \stackrel{\sigma}{\rightarrow} p_{\mathfrak{A}} \text { or } p_{1}=\perp \text { and } \varepsilon \stackrel{\sigma}{\rightarrow} \mathfrak{B} p_{2} \\
\Leftrightarrow & {[\text { compositionality of } \mathfrak{A}, \mathfrak{B}] } \\
& p_{2}=\perp \text { and } \exists \mathbf{q}_{\mathbf{1}} \in Q_{\mathfrak{A}}^{m} \cdot \mathbf{q}_{\mathbf{1}} \stackrel{\varphi}{\rightarrow}_{\mathfrak{A}} p_{1} \text { and } \\
& \forall 1 \leq i \leq m \cdot \varepsilon \stackrel{\tau_{i}}{\rightarrow} \mathbf{q}_{\mathfrak{A}}[i] \\
& \text { or } \\
& p_{1}=\perp \text { and } \exists \mathbf{q}_{\mathbf{2}} \in Q_{\mathfrak{B}}^{m} \cdot \mathbf{q}_{\mathbf{2}} \stackrel{\varphi}{\rightarrow}_{\mathfrak{B}} p_{2} \text { and } \\
& \forall 1 \leq i \leq m \cdot \varepsilon \stackrel{\tau_{i}}{\rightarrow} \mathbf{q}_{\mathbf{2}}[i] \\
\Leftrightarrow & {\left[\text { setting } \mathbf{q}=\left(\mathbf{q}_{\mathbf{1}}[1], \mathbf{q}_{\mathbf{2}}[1]\right) \ldots\left(\mathbf{q}_{\mathbf{1}}[m], \mathbf{q}_{\mathbf{2}}[m]\right), \text { construction of } \mathfrak{C}\right] } \\
& \exists \mathbf{q} \in Q^{m} \cdot \mathbf{q} \stackrel{\varphi}{\rightarrow} \mathfrak{C} p \text { and } \forall 1 \leq i \leq m \cdot \varepsilon \stackrel{\tau_{i}}{\rightarrow} \mathfrak{C} \mathbf{q}[i] .
\end{aligned}
$$

Lemma 12. Let $\mathfrak{A}, \mathfrak{B}$ be heap automata over $\mathrm{SH}_{\mathcal{C}}$. Then there exists a heap automaton $\mathfrak{C}$ over $\mathrm{SH}_{\mathcal{C}}$ such that $L(\mathfrak{C})=L(\mathfrak{A}) \cap L(\mathfrak{B})$.

Proof. Let $\mathfrak{A}, \mathfrak{B}$ be heap automata over $\mathrm{SH}_{\mathcal{C}}$ accepting $H$ and $K$. We construct a heap automaton $\mathfrak{C}=\left(Q, \mathrm{SH}_{\mathcal{C}}, \Delta, F\right)$ as follows:

$$
\begin{aligned}
Q \triangleq & Q_{\mathfrak{A}} \times Q_{\mathfrak{B}} \quad F \triangleq F_{\mathfrak{A}} \times F_{\mathfrak{B}} \\
\Delta: & \left(p_{1}, q_{1}\right) \ldots\left(p_{m}, q_{m}\right) \stackrel{\varphi}{\rightarrow} \mathfrak{C}\left(p_{0}, q_{0}\right) \Leftrightarrow \\
& p_{1} \ldots p_{m} \stackrel{\varphi}{\rightarrow}_{\mathfrak{A}} p_{0} \text { and } q_{1} \ldots q_{m} \stackrel{\varphi}{\rightarrow}_{\mathfrak{B}} q_{0},
\end{aligned}
$$

where $\varphi \in \mathrm{SH}_{\mathcal{C}}$ with $\left\|\Gamma^{\varphi}\right\|=m$. Decidability of $\Delta$ follows immediately from decidability of $\Delta_{\mathfrak{A}}$ and $\Delta_{\mathfrak{B}}$. Assuming $\mathfrak{C}$ satisfies the compositionality property, we first show that $L(\mathfrak{C})=L(\mathfrak{A}) \cap L(\mathfrak{B})$ :

$$
\tau \in L(\mathfrak{A}) \cap L(\mathfrak{B})
$$

$\Leftrightarrow$ [Definition of $\cap$ ] 


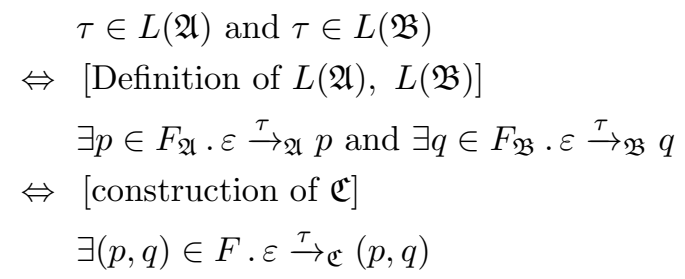

It remains to show the compositionality property, i.e., for each $p \in Q, \varphi \in \mathrm{SH}_{\mathcal{C}}$ with $\left\|\Gamma^{\varphi}\right\|=m$ and $\tau_{1}, \ldots, \tau_{m} \in \mathrm{RSH}_{\mathcal{C}}$ with $\sigma=\varphi\left[P_{1} / \tau_{1}, \ldots, P_{m} / \tau_{m}\right]$, we have

$$
\varepsilon \stackrel{\sigma}{\rightarrow} \mathfrak{C} p \Leftrightarrow \exists \mathbf{q} \in Q^{m} . \mathbf{q} \stackrel{\varphi_{\rightarrow}}{\rightarrow} p \text { and } \forall 1 \leq i \leq m . \varepsilon \stackrel{\tau_{i}}{\longrightarrow} \mathfrak{C} \mathbf{q}[i] .
$$

In the following proof, we write $p_{1}, p_{2}$ to denote the first and second component of $p \in Q$, respectively.

$$
\begin{aligned}
& \varepsilon \stackrel{\sigma}{\rightarrow} \mathfrak{C} p \\
\Leftrightarrow & {[\text { construction of } \mathfrak{C}] } \\
& \varepsilon \stackrel{\sigma}{\rightarrow}_{\mathfrak{A}} p_{1} \text { and } \varepsilon \stackrel{\sigma}{\rightarrow}{ }_{\mathfrak{B}} p_{2} \\
\Leftrightarrow & {[\text { compositionality of } \mathfrak{A}, \mathfrak{B}] } \\
& \exists \mathbf{q}_{\mathbf{1}} \in Q_{\mathfrak{A}}^{m} \cdot \mathbf{q}_{\mathbf{1}} \stackrel{\varphi}{\rightarrow}_{\mathfrak{A}} p_{1} \text { and } \\
& \forall 1 \leq i \leq m \cdot \varepsilon \stackrel{\tau_{i}}{\rightarrow} \mathfrak{A} \mathbf{q}_{\mathbf{1}}[i] \\
& \text { and } \\
& \exists \mathbf{q}_{\mathbf{2}} \in Q_{\mathfrak{B}}^{m} \cdot \mathbf{q}_{\mathbf{2}} \stackrel{\varphi}{\rightarrow}_{\mathfrak{B}} p_{2} \text { and } \\
& \forall 1 \leq i \leq m \cdot \varepsilon{\stackrel{\tau_{i}}{\rightarrow} \mathfrak{B}}_{\mathbf{q}_{\mathbf{2}}}[i] \\
\Leftrightarrow & {\left[\text { setting } \mathbf{q}=\left(\mathbf{q}_{\mathbf{1}}[1], \mathbf{q}_{\mathbf{2}}[1]\right) \ldots\left(\mathbf{q}_{\mathbf{1}}[m], \mathbf{q}_{\mathbf{2}}[m]\right),\right.} \\
& \quad \operatorname{construction} \text { of } \mathfrak{C}] \\
& \exists \mathbf{q} \in Q^{m} \cdot \mathbf{q} \stackrel{\varphi}{\rightarrow} \mathfrak{C} p \text { and } \forall 1 \leq i \leq m \cdot \varepsilon \stackrel{\tau_{i}}{\rightarrow} \mathfrak{C} \mathbf{q}[i]
\end{aligned}
$$

Lemma 13. Let $\mathfrak{A}$ be a heap automaton over $\mathrm{SH}_{\mathcal{C}}$. Then there exists a heap automaton $\mathfrak{C}$ over $\mathrm{SH}_{\mathcal{C}}$ such that $L(\mathfrak{C})=\mathrm{RSH}_{\mathcal{C}} \backslash L(\mathfrak{A})$.

Proof. We construct a heap automaton $\mathfrak{C}=\left(Q, \mathrm{SH}_{\mathcal{C}}, \Delta, F\right)$ accepting $\mathrm{RSH}_{\mathcal{C}} \backslash$ $L(\mathfrak{A})$ as follows:

$$
\begin{aligned}
& Q \triangleq 2^{Q_{\mathfrak{A}}} \quad F \triangleq\left\{X \subseteq Q_{\mathfrak{A}} \mid X \cap F_{\mathfrak{A}}=\emptyset\right\} \\
& \Delta: X_{1} \ldots X_{m} \stackrel{\varphi}{\rightarrow}_{\mathfrak{C}} X_{0} \Leftrightarrow \\
& \quad \forall q_{0} \in X_{0} . \forall 1 \leq i \leq m \cdot \exists q_{i} \in X_{i} . q_{1} \ldots q_{m} \stackrel{\varphi}{\rightarrow}_{\mathfrak{A}} q_{0} \\
& \quad \text { and } \\
& \quad \forall q_{0} \notin X_{0} . \forall 1 \leq i \leq m . \forall q_{i} \in X_{i} . \operatorname{not} q_{1} \ldots q_{m} \stackrel{\varphi}{\rightarrow}_{\mathfrak{A}} q_{0},
\end{aligned}
$$

where $\varphi \in \mathrm{SH}_{\mathcal{C}}$ with $\left\|\Gamma^{\varphi}\right\|=m$. Decidability of $\Delta$ follows immediately from decidability of $\Delta_{\mathfrak{A}}$. Assuming $\mathfrak{C}$ satisfies the compositionality property, we first 
show that $L(\mathfrak{C})=\mathrm{RSH}_{\mathcal{C}} \backslash L(\mathfrak{A})$ :

$$
\begin{aligned}
& \tau \in \mathrm{RSH}_{\mathcal{C}} \backslash L(\mathfrak{A}) \\
\Leftrightarrow & {\left[\text { Definition of } \operatorname{RSH}_{\mathcal{C}} \backslash L(\mathfrak{A})\right] } \\
& \forall p \in F_{\mathfrak{A}} \cdot \operatorname{not} \varepsilon \stackrel{\tau}{\rightarrow} \mathfrak{A} p \\
\Leftrightarrow & {[\text { Definition of } F] } \\
\Leftrightarrow & \exists X \in F . \forall p \in X . \varepsilon \stackrel{\tau}{\rightarrow}_{\mathfrak{A}} p \text { and } \forall p \notin X . \text { and } \varepsilon \stackrel{\tau}{\rightarrow}_{\mathfrak{A}} p \\
\Leftrightarrow & {[\text { construction of } \mathfrak{C}] } \\
\Leftrightarrow & \exists X \in F . \varepsilon \stackrel{\tau}{\rightarrow}_{\mathfrak{C}} X
\end{aligned}
$$

It remains to show the compositionality property, i.e., for each $X \in Q, \varphi \in \mathrm{SH}_{\mathcal{C}}$ with $\left\|\Gamma^{\varphi}\right\|=m$ and $\tau_{1}, \ldots, \tau_{m} \in \operatorname{RSH}_{\mathcal{C}}$ with $\sigma=\varphi\left[P_{1} / \tau_{1}, \ldots, P_{m} / \tau_{m}\right]$, we have

$$
\varepsilon \stackrel{\sigma}{\rightarrow} \mathfrak{C} p \Leftrightarrow \exists \mathbf{Y} \in Q^{m} . \mathbf{Y} \stackrel{\varphi}{\rightarrow} \mathfrak{C} X \text { and } \forall 1 \leq i \leq m . \varepsilon \stackrel{\tau_{i}}{\rightarrow} \mathfrak{C} \mathbf{Y}[i]
$$

Assume $\varepsilon \stackrel{\sigma}{\rightarrow} \mathfrak{C} X$. We choose $\mathbf{Y}$ such that for each $1 \leq i \leq m$, we have

$$
\mathbf{Y}[i] \triangleq\left\{q \in Q \mid \varepsilon{\stackrel{\tau_{i}}{\rightarrow}}_{\mathfrak{A}} q\right\} .
$$

Then $\varepsilon \stackrel{\tau_{i}}{\rightarrow} \mathfrak{C} \mathbf{Y}[i]$ and $\mathbf{Y} \stackrel{\varphi}{\rightarrow} \mathfrak{C} X$ hold immediately by construction and our choice of $\mathbf{Y}$.

For the converse direction assume there exists $\mathbf{Y} \in Q^{m}$ such that $\mathbf{Y} \stackrel{\varphi}{\rightarrow}_{\mathfrak{C}} X$ and for each $1 \leq i \leq m$, we have $\varepsilon \stackrel{\tau_{i}}{\longrightarrow} \mathfrak{C} \mathbf{Y}[i]$. Two cases arise for each $p \in Q_{\mathfrak{A}}$. First, assume $p \in X$. By construction, there exists a $q_{i} \in \mathbf{Y}[i]$ for each $1 \leq i \leq m$ such that $q_{1} \ldots q_{m} \stackrel{\varphi}{\rightarrow}_{\mathfrak{A}} p$ and $\varepsilon \stackrel{\tau_{i}}{\rightarrow} \mathfrak{A} q_{i}, 1 \leq i \leq m$. Since $\mathfrak{A}$ is a heap automaton this implies $\varepsilon \stackrel{\sigma}{\rightarrow} \mathfrak{A} p$.

Second, assume $p \notin X$. Then for each choice of $q_{i} \in q_{i} \in \mathbf{Y}[i], 1 \leq i \leq m$, we know by construction of $\Delta$ that $q_{1} \ldots q_{m} \stackrel{\varphi}{\rightarrow} \mathfrak{A} p$ as well as $\varepsilon \stackrel{\tau_{i}}{\rightarrow} \mathfrak{A} q_{k}$ do not hold. Since $\mathfrak{A}$ is a heap automaton this implies that $\varepsilon \stackrel{\sigma}{\rightarrow}_{\mathfrak{A}} p$ does not hold.

Putting both cases together yields $\varepsilon \stackrel{\sigma}{\rightarrow} \mathfrak{C} X$.

\section{A.7 Proof of Theorem 1}

The following lemma is essential to show that our construction of $\Psi$ is correct.

Lemma 14. Let $\Phi, \Psi$ and $\mathfrak{A}$ be as in Theorem 1. Then, for each $P \in \operatorname{Pred}(\Phi)$ and $q \in Q_{\mathfrak{A}}$, we have

$$
\tau \in \mathbb{U}_{\Psi}\left((P, q) \mathbf{x}_{0}\right) \quad \text { iff } \quad \tau \in \mathbb{U}_{\Phi}\left(P \mathbf{x}_{0}\right) \text { and } \varepsilon \stackrel{\tau}{\rightarrow} \mathfrak{A} q .
$$

Proof. Let $P \in \operatorname{Pred}(\Phi)$ and $q \in Q_{\mathfrak{A}}$. By induction on the height $k$ of unfolding trees $t$, we show

$$
\begin{gathered}
t \in \mathbb{T}_{\Psi}\left((P, q) \mathbf{x}_{0}\right) \Leftrightarrow \exists t^{\prime} \in \mathbb{T}_{\Phi}\left(P \mathbf{x}_{0}\right) \cdot \llbracket t^{\prime} \rrbracket=\llbracket t \rrbracket \\
\text { and } \varepsilon \stackrel{\llbracket t \rrbracket}{\longrightarrow} \mathfrak{A} q .
\end{gathered}
$$


I.B. Note that, by Definition of unfolding trees, $t(\varepsilon)=(P, q) \mathbf{x}_{0}$. If $k=0$ there is nothing to show. Thus assume $k=1$. Then

$$
\begin{aligned}
& \llbracket t \rrbracket \\
= & {[\text { Definition of } \llbracket t \rrbracket] } \\
& (P, q) \mathbf{x}_{0}\left[(P, q) /\left.\llbracket t\right|_{1} \rrbracket\right] \\
= & {[\text { Definition of predicate replacement }] } \\
= & \llbracket t t_{1} \rrbracket \\
= & {\left[k=1 \text { implies }\left.\llbracket t\right|_{1} \rrbracket=t(1) \in \mathrm{RSH}\right] } \\
= & t(1) .
\end{aligned}
$$

Hence

$$
\begin{aligned}
& t \in \mathbb{T}_{\Psi}\left((P, q) \mathbf{x}_{0}\right) \\
\Leftrightarrow & {[\text { Definition } 2, k=1] } \\
& (P, q) \Leftarrow t(1) \in \Psi \\
\Leftrightarrow & {[\text { Construction of } \Psi] } \\
& P \Leftarrow t(1) \in \Phi \text { and } \varepsilon \stackrel{q}{\rightarrow} \mathfrak{A} t(1) \\
\Leftrightarrow & {[\text { Definition } 2] } \\
& \exists t^{\prime} \in \mathbb{T}_{\Phi}\left(P \mathbf{x}_{0}\right) \cdot \llbracket t \rrbracket=t(1) \text { and } \varepsilon \stackrel{q}{\rightarrow}_{\mathfrak{A}} t(1) \\
\Leftrightarrow & {[\llbracket t \rrbracket=t(1)] } \\
& \exists t^{\prime} \in \mathbb{T}_{\Phi}\left(P \mathbf{x}_{0}\right) \cdot \llbracket t^{\prime} \rrbracket=\llbracket t \rrbracket \text { and } \varepsilon \stackrel{q}{\rightarrow}_{\mathfrak{A}} \llbracket t \rrbracket .
\end{aligned}
$$

I.H. Assume for an arbitrary, but fixed natural number $k$ that for each predicate symbol $P \in \operatorname{Pred}(\Phi)$, each state $q \in \mathfrak{A}$ and each unfolding tree $t \in \mathbb{T}_{\Psi}\left((P, q) \mathbf{x}_{0}\right)$ of height at most $k$, we have

$$
\begin{gathered}
t \in \mathbb{T}_{\Psi}\left((P, q) \mathbf{x}_{0}\right) \Leftrightarrow \exists t^{\prime} \in \mathbb{T}_{\Phi}\left(P \mathbf{x}_{0}\right) \cdot \llbracket t^{\prime} \rrbracket=\llbracket t \rrbracket \\
\text { and } \varepsilon \stackrel{\llbracket t \rrbracket}{\longrightarrow} \mathfrak{A} q .
\end{gathered}
$$

I.S. Let $t \in \mathbb{T}_{\Psi}\left((P, q) \mathbf{x}_{0}\right)$ be an unfolding tree of height $k+1$. Then $t(1)$ is not a reduced symbolic heap (otherwise the height of $t$ would be 1 ). Thus, given some $m \geq 1$, we may assume that

$$
t(1)=\exists \mathbf{z} \cdot \Sigma *\left(P_{1}, q_{1}\right) \mathbf{x}_{1} * \ldots *\left(P_{m}, q_{m}\right) \mathbf{x}_{m}: \Pi
$$

Then

$$
\begin{aligned}
& \llbracket t \rrbracket \\
= & {[\text { Definition of } \llbracket t \rrbracket] } \\
& t(\varepsilon)\left[(P, q) /\left.\llbracket t\right|_{1} \rrbracket\right] \\
= & {\left[t(\varepsilon)=(P, q) \mathbf{x}_{0}, \text { definition of predicate replacement }\right] }
\end{aligned}
$$




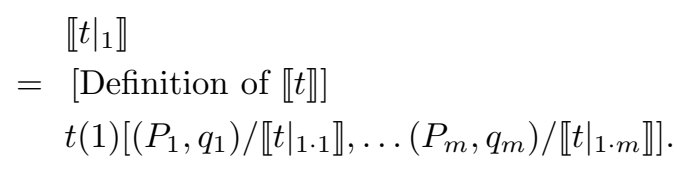

Now, for each $1 \leq i \leq m,\left.t\right|_{1 \cdot i}$ is an unfolding tree of $\left(P_{i}, q_{i}\right) \mathbf{x}_{0}$ of height at most $k$. Thus, by I.H. we know that

$$
\begin{gathered}
\left.t\right|_{1 \cdot i} \in \mathbb{T}_{\Psi}\left(\left(P_{i}, q_{i}\right) \mathbf{x}_{0}\right) \Leftrightarrow \exists t_{i} \in \mathbb{T}_{\Phi}\left(P_{i} \mathbf{x}_{0}\right) \cdot \llbracket t_{i} \rrbracket=\left.\llbracket t\right|_{1 \cdot i} \rrbracket \\
\text { and } \varepsilon \stackrel{\llbracket t_{1 \cdot i} \rrbracket_{\mathfrak{A}}}{\longrightarrow} q_{i} .
\end{gathered}
$$

Then

$$
\begin{aligned}
& t \in \mathbb{T}_{\Psi}\left((P, q) \mathbf{x}_{0}\right) \\
& \Leftrightarrow \quad[\text { Definition } 2, k>1] \\
& (P, q) \Leftarrow t(1) \in \Psi \\
& \text { and } \forall 1 \leq i \leq m .\left.t\right|_{i} \in \mathbb{T}_{\Psi}\left(\left(P_{i}, q_{i}\right) \mathbf{x}_{0}\right) \\
& \Leftrightarrow \quad \text { Construction of } \Psi] \\
& P \Leftarrow\left(\exists \mathbf{z} . \Sigma * P_{1} \mathbf{x}_{1} * \ldots * P_{m} \mathbf{x}_{m}: \Pi\right) \in \Phi \\
& \text { and } q_{1} \ldots q_{m} \stackrel{t(1)}{\longrightarrow} \mathfrak{A} q \\
& \text { and } \forall 1 \leq i \leq m .\left.t\right|_{i} \in \mathbb{T}_{\Psi}\left(\left(P_{i}, q_{i}\right) \mathbf{x}_{0}\right) \\
& \Leftrightarrow[\text { I.H. }] \\
& P \Leftarrow\left(\exists \mathbf{z} . \Sigma * P_{1} \mathbf{x}_{1} * \ldots * P_{m} \mathbf{x}_{m}: \Pi\right) \in \Phi \\
& \text { and } q_{1} \ldots q_{m} \stackrel{t(1)}{\longrightarrow} \mathfrak{A}_{\mathfrak{A}} q \\
& \text { and } \forall 1 \leq i \leq m . \exists t_{i} \in \mathbb{T}_{\Phi}\left(P_{i} \mathbf{x}_{0}\right) \cdot \llbracket t_{i} \rrbracket=\left.\llbracket t\right|_{1 \cdot i} \rrbracket \\
& \text { and } \varepsilon \stackrel{\left.\llbracket t\right|_{1 . i} \rrbracket}{\longrightarrow} \mathfrak{A} q_{i} \text {. } \\
& \Leftrightarrow\left[\text { Definition 2, } t^{\prime}(1) \triangleq \exists \mathbf{z} \cdot \Sigma * P_{1} \mathbf{x}_{1} * \ldots * P_{m} \mathbf{x}_{m}: \Pi\right] \\
& \exists t^{\prime} \in \mathbb{T}_{\Phi}\left(P \mathbf{x}_{0}\right) . \llbracket t^{\prime} \rrbracket=t^{\prime}(1)\left[P_{1} /\left.\llbracket t\right|_{1 \cdot 1} \rrbracket, \ldots, P_{m} /\left.\llbracket t\right|_{1 \cdot m} \rrbracket\right] \\
& \text { and } q_{1} \ldots q_{m} \stackrel{t(1)}{\longrightarrow} \mathfrak{A} q \\
& \text { and } \forall 1 \leq i \leq m \cdot \varepsilon \stackrel{\left.\llbracket t\right|_{1 \cdot i} \rrbracket}{\longrightarrow} \mathfrak{A} q_{i} \\
& \Leftrightarrow \text { [Definition } 6] \\
& \exists t^{\prime} \in \mathbb{T}_{\Phi}\left(P \mathbf{x}_{0}\right) . \llbracket t^{\prime} \rrbracket=t^{\prime}(1)\left[P_{1} /\left.\llbracket t\right|_{1 \cdot 1} \rrbracket, \ldots, P_{m} /\left.\llbracket t\right|_{1 \cdot m} \rrbracket\right] \\
& \text { and } \varepsilon \stackrel{\left.\llbracket t\right|_{1} \rrbracket}{\longrightarrow} \mathfrak{A} q \\
& \Leftrightarrow \text { [applying } \\
& \exists t^{\prime} \in \mathbb{T}_{\Phi}\left(P \mathbf{x}_{0}\right) \cdot \llbracket t^{\prime} \rrbracket=\llbracket t \rrbracket \text { and } \varepsilon \stackrel{\llbracket t \rrbracket}{\longrightarrow}_{\mathfrak{A}} q .
\end{aligned}
$$

To finish the proof, note that

$$
\tau \in \mathbb{U}_{\Psi}\left((P, q) \mathbf{x}_{0}\right)
$$




$$
\begin{aligned}
\Leftrightarrow & {[\text { Definition } 4] } \\
& \exists t \in \mathbb{T}_{\Psi}\left((P, q) \mathbf{x}_{0}\right) \cdot \llbracket t \rrbracket=\tau \\
\Leftrightarrow & {[\text { previously shown proposition] }} \\
& \exists t^{\prime} \in \mathbb{T}_{\Phi}\left(P \mathbf{x}_{0}\right) . \llbracket t^{\prime} \rrbracket=\tau \text { and } \varepsilon \stackrel{\tau}{\rightarrow}_{\mathfrak{A}} q \\
\Leftrightarrow & {[\text { Definition } 4] } \\
& \tau \in \mathbb{U}_{\Phi}\left(P \mathbf{x}_{0}\right) \text { and } \varepsilon \stackrel{\tau}{\rightarrow}_{\mathfrak{A}} q . \square
\end{aligned}
$$

We are now in a position to prove Theorem 1.

Proof (Proof of Theorem 1). It remains to show that for each $P \in \operatorname{Pred}(\Phi)$, we have $\mathbb{U}_{\Psi}\left(P \mathbf{x}_{0}\right)=\mathbb{U}_{\Phi}\left(P \mathbf{x}_{0}\right) \cap L(\mathfrak{A})$. By Lemma 14, we know that for each $P \in \operatorname{Pred}(\Phi)$ and $q \in Q_{\mathfrak{A}}$, we have

$$
\tau \in \mathbb{U}_{\Psi}\left((P, q) \mathbf{x}_{0}\right) \quad \text { iff } \quad \tau \in \mathbb{U}_{\Phi}\left(P \mathbf{x}_{0}\right) \text { and } \varepsilon \stackrel{\tau}{\rightarrow}_{\mathfrak{A}} q .
$$

Then

$$
\begin{aligned}
& \tau \in \mathbb{U}_{\Psi}\left(P \mathbf{x}_{0}\right) \\
\Leftrightarrow & {[\text { Definition } 4] } \\
& \exists t \in \mathbb{T}_{\Psi}\left(P \mathbf{x}_{0}\right) \cdot \llbracket t \rrbracket=\tau \\
\Leftrightarrow & {\left[\llbracket t \rrbracket=P \mathbf{x}_{0}\left[P \mathbf{x}_{0} /\left.\llbracket t\right|_{1} \rrbracket\right]=\left.\llbracket t\right|_{1} \rrbracket\right] } \\
& \left.\exists t \in \mathbb{T}_{\Psi}\left(P \mathbf{x}_{0}\right) \cdot \llbracket t\right|_{1} \rrbracket=\tau \\
\Leftrightarrow & {\left[P \Leftarrow(P, q) \in \Psi \Leftrightarrow q \in F_{\mathfrak{A}} \text { and } t(\varepsilon)=P \mathbf{x}_{0}\right.} \\
& \left.\quad \text { implies } \exists q \in F_{\mathfrak{A}} . t(1)=(P, q) \mathbf{x}_{0}\right] \\
& \exists q \in F_{\mathfrak{A}} \cdot \exists t^{\prime} \in \mathbb{T}_{\Psi}\left((P, q) \mathbf{x}_{0}\right) \cdot \llbracket t^{\prime} \rrbracket=\tau \\
\Leftrightarrow & {[\text { Definition } 4] } \\
& \exists q \in F_{\mathfrak{A}} \cdot \tau \in \mathbb{U}_{\Psi}\left((P, q) \mathbf{x}_{0}\right) \\
\Leftrightarrow & {[\operatorname{applying}(\star)] } \\
& \exists q \in F_{\mathfrak{A}} \cdot \tau \in \mathbb{U}_{\Phi}\left(P \mathbf{x}_{0}\right) \text { and } \varepsilon \stackrel{\tau}{\rightarrow} \mathfrak{A} q . \\
\Leftrightarrow & {\left[\tau \in L(\mathfrak{A}) \Leftrightarrow \exists q \in F_{\mathfrak{A}} \cdot \varepsilon \stackrel{\tau}{\rightarrow} \mathfrak{A} q\right] } \\
& \tau \in \mathbb{U}_{\Phi}\left(P \mathbf{x}_{0}\right) \text { and } \tau \in L(\mathfrak{A}) \\
\Leftrightarrow & {[x \in A \wedge x \in B \Leftrightarrow x \in A \cap B] } \\
& \tau \in \mathbb{U}_{\Phi}\left(P \mathbf{x}_{0}\right) \cap \tau \in L(\mathfrak{A}) .
\end{aligned}
$$

\section{A.8 Details on definite relationships}

This section briefly shows how to compute the definite relationships $=_{\tau}, \neq_{\tau}$, alloc $(\tau)$ and $x \mapsto_{\tau} y$ introduced at the beginning of Section 4 . 
Definition 16. Let $\tau=\exists \mathbf{z} . \Sigma: \Pi \in \mathrm{RSH}$. Then the completion of $\tau$ is given by the symbolic heap complete $(\tau) \triangleq \exists \mathbf{z} . \Sigma$ : closure $(\Lambda)$, where closure $(\Lambda)$ denotes the reflexive, symmetric, and transitive closure w.r.t. $=$ and the symmetric closure w.r.t. $\neq$ of the set of pure formulas $\Lambda$ given by

$$
\begin{aligned}
\Pi \cup\{x \neq \text { null } \mid x \mapsto(-) \text { occurs in } \Sigma\} \\
\cup\{x \neq y \mid x \mapsto(-), y \mapsto(-) \text { distinct points-to assertions in } \Sigma\} .
\end{aligned}
$$

Moreover, if closure $(\Lambda)$ is inconsistent, i.e., $x \neq x$ or null $\neq$ null is contained in closure $(\Lambda)$, we define closure $(\Lambda)$ to be the set of all pure formulas over $\operatorname{Var}(\tau)$.

Clearly, $\Lambda$ is computable in at most quadratic time. Then computing closure $(\Lambda)$ is possible in at most cubic time. Finally, checking for inconsistencies can be performed in linear time. Hence, the completion of a reduced symbolic heap is computable in polynomial time. The following lemma provides a characterization of each of the definite relationships between variables introduced in Section 4 .

Lemma 15. Let $\tau \in \mathrm{RSH}$ and $x, y \in \operatorname{Var}(\tau)$. Moreover, let $\sigma=\operatorname{complete}(\tau)$.

Then

$$
\begin{aligned}
& -x=_{\tau} y \text { iff } x=y \in \Pi^{\sigma}, \\
& -x \neq \neq_{\tau} y \text { iff } x \neq y \in \Pi^{\sigma}, \\
& -x \in \text { alloc }(\tau) \text { iff } x \neq x \in \Pi^{\sigma} \text { or there exists } z \mapsto\left({ }_{-}\right) \text {in } \Sigma^{\sigma} \text { such that } \\
& \quad x=z \in \Pi^{\sigma}, \\
& -x \mapsto_{\tau} y \text { iff } x \neq x \in \Pi^{\sigma} \text { or there exists } z_{1} \mapsto\left(\left(-, z_{2},-\right)\right) \text { in } \Sigma^{\sigma} \text { such that } \\
& \quad x=z_{1} \in \Pi^{\sigma} \text { and } y=z_{2} \in \Pi^{\sigma} \text {. }
\end{aligned}
$$

In particular, the right-hand side of each of these characterizations is easily computable in polynomial time once a completion of a symbolic heap has been computed.

Proof. Let $\tau=\exists \mathbf{z} . \Sigma: \Pi$. Then, for each $(s, h) \in \operatorname{Models}(\tau)$, we know by the SL semantics (cf. Figure 1) that there exists $\mathbf{v} \in V a l^{\|\mathbf{z}\|}$ such that $s^{\prime}=$ $s[\mathbf{z} \mapsto \mathbf{v}], \operatorname{dom}(h)=\left\{s^{\prime}(x) \mid x \mapsto(\mathbf{y})\right.$ in $\left.\Sigma\right\}, h\left(s^{\prime}(x)\right)=s^{\prime}(\mathbf{y})$ for each $x \mapsto(\mathbf{y})$ in $\Sigma$, and for each $x \sim y \in \Pi, s^{\prime}(x) \sim s^{\prime}(y)$. The same holds for $\sigma$ except that $\Pi$ is substituted by closure $(\Lambda)$ (although each additional pure formula is already implied by the conditions from above). Thus, $\operatorname{Models}(\tau)=\operatorname{Models}(\sigma)$. Furthermore, by construction of $\Pi^{\sigma}=\operatorname{closure}(\Lambda)$, we have $x={ }_{\sigma} y$ iff $x=y \in \Pi^{\sigma}$ and $x \neq_{\sigma}$ iff $x \neq y \in \Pi^{\sigma}$. Then, each of the properties in Lemma 15 is easy to verify.

\section{A.9 Appendix to Section 4}

\section{A.9.1 Proof of Lemma 3}

Note that some statements are broken down into separate lemmas which are shown immediately afterwards. 
Lemma 3. For all $\alpha \in \mathbb{N}_{>0}$ and all sets $A \subseteq \mathbf{x}_{0}, \Pi \in \operatorname{Pure}\left(\mathbf{x}_{0}\right)$, there is a heap automaton over $\mathrm{SH}_{F V \leq \alpha}$ accepting $\operatorname{TRACK}(\alpha, A, \Pi)$.

Proof. It suffices to show that the heap automaton $\mathfrak{A}_{\text {TRACK }}$ constructed in Definition 10 satisfies the compositionality property and accepts $\operatorname{TRACK}(\alpha, A, \Pi)$. We first show the latter, i.e., $L\left(\mathfrak{A}_{\mathrm{TRACK}}\right)=\operatorname{TRACK}(\alpha, A, \Pi)$. Let $\tau \in \mathrm{RSH}_{\mathrm{FV} \leq \alpha}$. Then

$$
\begin{aligned}
& \tau \in L\left(\mathfrak{A}_{\mathrm{TRACK}}\right) \\
\Leftrightarrow & {\left[\text { Definition } L\left(\mathfrak{A}_{\mathrm{TRACK}}\right)\right] } \\
& \exists\left(A^{\prime}, \Pi^{\prime}\right) \in F_{\mathfrak{A}_{\mathrm{TRACK}}} \cdot \varepsilon \stackrel{\tau}{\rightarrow}_{\mathfrak{A}_{\text {TRACK }}}\left(A^{\prime}, \Pi^{\prime}\right) \\
\Leftrightarrow & {\left[F_{\mathfrak{A}_{\text {TRACK }}}=\{(A, \Pi)\}\right] } \\
& \varepsilon{\stackrel{\tau}{\rightarrow} \mathfrak{A}_{\mathrm{TRACK}}}(A, \Pi) \\
\Leftrightarrow & {[\text { Definition } \Delta] } \\
& \forall x, y \in \mathbf{x}_{0}^{\tau} \cdot x \in A \leftrightarrow x \in \operatorname{alloc}(\operatorname{compress}(\tau, \varepsilon)) \\
& \quad \text { and }(x \sim y) \in \Pi \leftrightarrow x \sim_{\operatorname{compress}(\tau, \varepsilon)} y \\
\Leftrightarrow & {[\text { Definition } 10: \operatorname{compress}(\tau, \varepsilon)=\tau] } \\
& \forall x, y \in \mathbf{x}_{0}^{\tau} \cdot x \in A \leftrightarrow x \in \operatorname{alloc}(\tau) \\
& \quad \text { and }(x \sim y) \in \Pi \leftrightarrow x \sim_{\tau} y \\
\Leftrightarrow & {[\text { Definition } \operatorname{TRACK}(\alpha, A, \Pi)] } \\
& \tau \in \operatorname{TRACK}(\alpha, A, \Pi) .
\end{aligned}
$$

The compositionality property of $\mathfrak{A}_{\mathrm{TRACK}}$ is verified separately in Lemma 17 , which is proven in the remainder of this section.

Lemma 17. Let $\varphi \in \mathrm{SH}_{F V \leq \alpha}$ with $\left\|\Gamma^{\varphi}\right\|=m \geq 0$. Moreover, for each $1 \leq i \leq$ $m$, let $\tau_{i} \in \mathrm{RSH}_{F V \leq \alpha}$ with $\left\|\mathbf{x}_{0}^{\tau_{i}}\right\|=\left\|\mathbf{x}_{i}^{\varphi}\right\|$. Then, for $\tau=\varphi\left[P_{1} / \tau_{1}, \ldots P_{m} / \tau_{m}\right]$, we have $\varepsilon \stackrel{\tau}{\rightarrow}_{\mathfrak{A}_{\mathrm{TraCK}}}\left(A_{0}, \Pi_{0}\right)$ if and only if there exist $\left(A_{1}, \Pi_{1}\right), \ldots,\left(A_{m}, \Pi_{m}\right) \in$ $Q_{\mathfrak{A}_{\mathrm{TRACK}}}$ such that

$$
\left(A_{1}, \Pi_{1}\right) \ldots\left(A_{m}, \Pi_{m}\right) \stackrel{\varphi}{\rightarrow}_{\mathfrak{A}_{\text {TRACK }}}\left(A_{0}, \Pi_{0}\right)
$$

and, for each $1 \leq i \leq m$, we have $\varepsilon \stackrel{\tau_{i}}{\rightarrow} \mathfrak{A}_{\text {тRAск }}\left(A_{i}, \Pi_{i}\right)$.

Proof. Recall from Definition 10 that

$$
\operatorname{compress}(\varphi, \mathbf{q}) \triangleq \varphi\left[P_{1} / \operatorname{kernel}\left(P_{1} \mathbf{x}_{1}, \mathbf{q}[1]\right), \ldots, P_{m} / \operatorname{kernel}\left(P_{m} \mathbf{x}_{m}, \mathbf{q}[m]\right)\right] .
$$

Moreover, for each $1 \leq i \leq m$, let

$$
\begin{aligned}
& A_{i} \triangleq\left\{\mathbf{x}_{0}[\ell] \mid \mathbf{x}_{0}^{\tau_{i}}[\ell] \in \operatorname{alloc}\left(\tau_{i}\right)\right\}, \text { and } \\
& \Pi_{i} \triangleq\left\{\mathbf{x}_{0}[\ell] \sim \mathbf{x}_{0}[k] \mid \mathbf{x}_{0}^{\tau_{i}}[\ell] \sim_{\tau_{i}} \mathbf{x}_{0}^{\tau_{i}}[k]\right\} .
\end{aligned}
$$

Then

$$
\varepsilon \stackrel{\tau}{\rightarrow}_{\mathfrak{A}_{\text {TRACK }}}\left(A_{0}, \Pi_{0}\right)
$$




$$
\begin{aligned}
& \left.\Leftrightarrow \quad \text { Definition of } \Delta \text { and }\left\|\Gamma^{\tau}\right\|=0\right] \\
& \forall x, y \in \mathbf{x}_{0} . x \in A_{0} \text { iff } x^{\varphi} \in \operatorname{alloc}(\tau) \\
& \text { and } x \sim y \in \Pi_{0} \text { iff } x^{\varphi} \sim_{\tau} y^{\varphi} \\
& \left.\Leftrightarrow \quad \text { choice of } A_{i} \text { and } \Pi_{i} \text { for each } 1 \leq i \leq m\right] \\
& \exists \mathbf{q}=\left(A_{1}, \Pi_{1}\right) \ldots\left(A_{m}, \Pi_{m}\right) \in Q_{\mathfrak{A}_{\text {TRACK }}}^{m} . \forall x, y \in \mathbf{x}_{0} . \\
& x \in A_{0} \text { iff } x^{\varphi} \in \operatorname{alloc}(\tau) \\
& \text { and } x \sim y \in \Pi_{0} \text { iff } x^{\varphi} \sim_{\tau} y^{\varphi} \\
& \text { and } \forall 1 \leq i \leq m \cdot \varepsilon{\stackrel{\tau_{i}}{\rightarrow}}_{\mathfrak{A}_{\mathrm{TACK}}}\left(A_{i}, \Pi_{i}\right) \\
& \Leftrightarrow \text { [Lemma 19] } \\
& \exists \mathbf{q}=\left(A_{1}, \Pi_{1}\right) \ldots\left(A_{m}, \Pi_{m}\right) \in Q_{\mathfrak{A}_{\text {TRACK }}}^{m} . \forall x, y \in \mathbf{x}_{0} . \\
& x \in A_{0} \text { iff } x^{\varphi} \in \operatorname{alloc}(\operatorname{compress}(\varphi, \mathbf{q})) \\
& \text { and } x \sim y \in \Pi_{0} \text { iff } x^{\varphi} \sim_{\operatorname{compress}(\varphi, \mathbf{q})} y^{\varphi} \\
& \text { and } \forall 1 \leq i \leq m \cdot \varepsilon{\stackrel{\tau_{i}}{\rightarrow}}_{\mathfrak{A}_{\mathrm{TRACK}}}\left(A_{i}, \Pi_{i}\right) \\
& \Leftrightarrow \text { [Definition of } \Delta] \\
& \begin{aligned}
\exists \mathbf{q}= & \left(A_{1}, \Pi_{1}\right) \ldots\left(A_{m}, \Pi_{m}\right) \in Q_{\mathfrak{A l}_{\text {TRACK }}^{m}}^{m} . \\
& \left(A_{1}, \Pi_{1}\right) \ldots\left(A_{m}, \Pi_{m}\right) \stackrel{\varphi}{\rightarrow}_{\mathfrak{A}}\left(A_{0}, \Pi_{0}\right) \\
& \text { and } \forall 1 \leq i \leq m . \varepsilon \stackrel{\tau_{i}}{\rightarrow} \mathfrak{A}_{\mathrm{TAACK}}\left(A_{i}, \Pi_{i}\right) .
\end{aligned}
\end{aligned}
$$

It remains to show that Lemma 19. The crux of the proof of Lemma 19 is the following observation.

Lemma 18. Let $\varphi=\exists \mathbf{z} . \Sigma * P_{1} \mathbf{x}_{1}: \Pi \in \mathrm{SH}_{F V \leq \alpha}$. Moreover, let $\tau \in \mathrm{RSH}$ with $\left\|\mathbf{x}_{0}^{\tau}\right\|=\left\|\mathbf{x}_{1}^{\varphi}\right\|, A=\left\{y \in \mathbf{x}_{0} \mid y^{\tau} \in \operatorname{alloc}(\tau)\right\}$ and $\Lambda=\left\{x \sim y \mid x^{\tau} \sim_{\tau} y^{\tau}\right\}$. Then, for each $x, y \in \operatorname{Var}(\varphi)$, we have

$$
\begin{array}{rll}
x \sim_{\varphi\left[P_{1} / \tau\right]} y & \text { iff } & x \sim_{\varphi\left[P_{1} / \operatorname{kernel}\left(P_{1} \mathbf{x}_{1},(A, \Lambda)\right)\right]} y, \quad \text { and } \\
x \in \operatorname{alloc}\left(\varphi\left[P_{1} / \tau\right]\right) & \text { iff } \quad x \in \operatorname{alloc}\left(\varphi\left[P_{1} / \operatorname{kernel}\left(P_{1} \mathbf{x}_{1},(A, \Lambda)\right)\right]\right),
\end{array}
$$

where kernel $\left(P_{1} \mathbf{x}_{1},(A, \Lambda)\right)$ is defined in Section 4 (above Definition 10).

Proving this lemma in turn relies on some auxiliary results, in particular, we need Lemma 22 and Lemma 23, which are proven at the end of this section.

Proof.

$$
\begin{aligned}
& x \sim_{\varphi\left[P_{1} / \tau\right]} y \\
\Leftrightarrow & {\left[\text { Definition of } \sim_{\varphi\left[P_{1} / \tau\right]}\right] } \\
& \forall(s, h) \cdot s, h \models_{\emptyset} \varphi\left[P_{1} / \tau\right] \text { implies } s(x) \sim s(y) \\
\Leftrightarrow & {[\operatorname{logic}] } \\
& \forall(s, h) \cdot\left(\exists h^{\prime} \cdot s, h^{\prime} \models_{\emptyset} \varphi\left[P_{1} / \tau\right]\right) \text { implies } s(x) \sim s(y)
\end{aligned}
$$




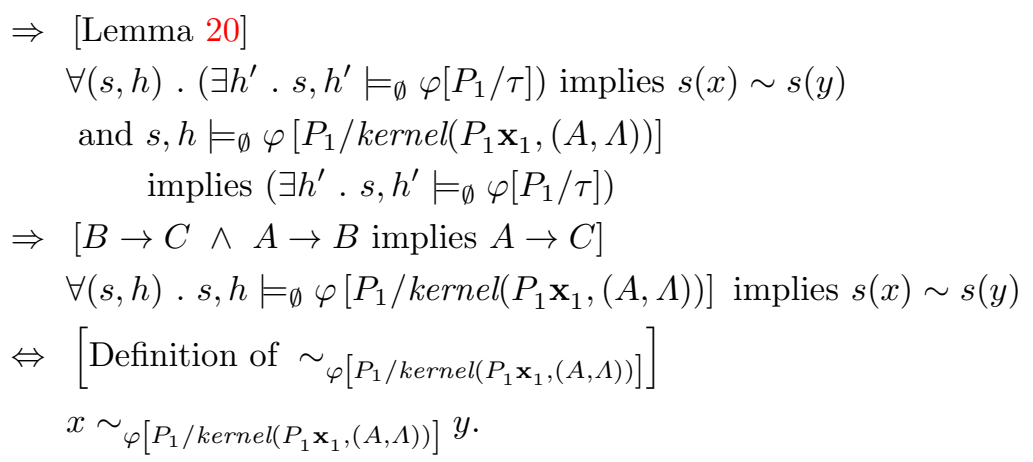

Furthermore, we obtain

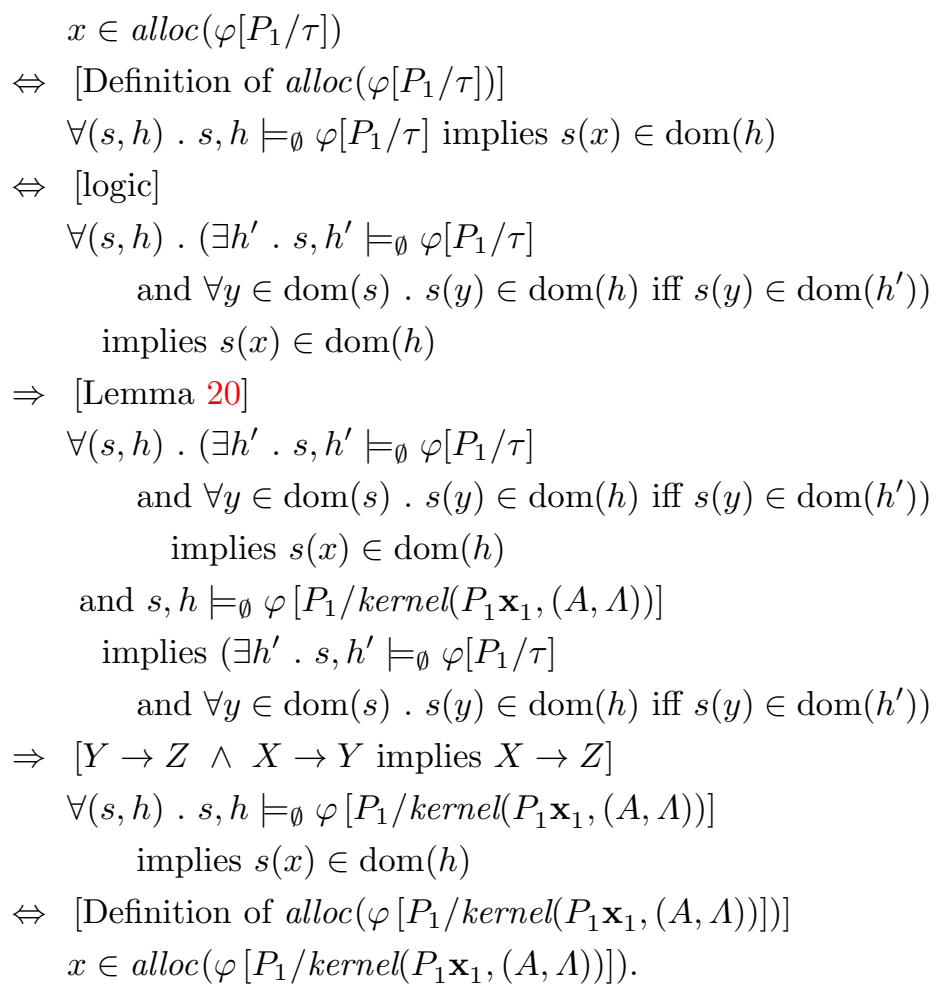

The proof of the converse direction is analogous, where the main difference is consists of using the property

$$
\begin{aligned}
s, h \models \emptyset \varphi\left[P_{1} / \tau\right] \text { implies } \exists h^{\prime} . s, h^{\prime} \models_{\emptyset} \varphi\left[P_{1} / \operatorname{kernel}\left(P_{1} \mathbf{x}_{1},(A, \Lambda)\right)\right] \\
\\
\quad \text { and } \forall y \in \operatorname{dom}(s) . s(y) \in \operatorname{dom}(h) \text { iff } s(y) \in \operatorname{dom}\left(h^{\prime}\right)
\end{aligned}
$$

instead of Lemma 20. This property is shown analogous to Lemma 20 except that we apply Lemma 23 instead of Lemma 22. 
Lemma 19. Let $\varphi \in \mathrm{SH}_{F V \leq \alpha}$ with $\left\|\Gamma^{\varphi}\right\|=m \geq 0$. For each $1 \leq i \leq m$, let $\tau_{i} \in \mathrm{RSH}_{F V \leq \alpha}$ with $\left\|\mathbf{x}_{0}^{\tau_{i}}\right\|=\left\|\mathbf{x}_{i}^{\varphi}\right\|, A_{i}=\left\{y \in \mathbf{x}_{0} \mid y^{\tau_{i}} \in \operatorname{alloc}\left(\tau_{i}\right)\right\}$ and $\Pi_{i}=\left\{x \sim y \mid x^{\tau_{i}} \sim_{\tau_{i}} y^{\tau_{i}}\right\}$. Moreover, let

$$
\begin{aligned}
\mathbf{q} & \triangleq\left(A_{1}, \Pi_{1}\right) \ldots\left(A_{m}, \Pi_{m}\right), \\
\tau & \triangleq \varphi\left[P_{1} / \tau_{1}, \ldots, P_{m} / \tau_{m}\right], \text { and } \\
\operatorname{compress}(\varphi, \mathbf{q}) & \triangleq \varphi\left[P_{1} / \operatorname{kernel}\left(P_{1} \mathbf{x}_{1}, \mathbf{q}[1]\right), \ldots, P_{m} / \operatorname{kernel}\left(P_{m} \mathbf{x}_{m}, \mathbf{q}[m]\right)\right] .
\end{aligned}
$$

Then, for each $x, y \in \operatorname{Var}(\varphi)$, we have

$$
\begin{aligned}
& x \in \operatorname{alloc}(\tau) \text { iff } x \in \operatorname{alloc}(\operatorname{compress}(\varphi, \mathbf{q})), \text { and } \\
& x \sim_{\tau} y \text { iff } x \sim_{\operatorname{compress}(\varphi, \mathbf{q})} y .
\end{aligned}
$$

Proof. We apply Lemma 18 iteratively to

$$
\begin{aligned}
\psi_{0}= & \varphi\left[\mathbf{P}_{\mathbf{1}} / \tau_{\mathbf{1}}, P_{2} / \tau_{2}, \ldots, P_{m} / \tau_{m}\right]=\tau \\
\psi_{1}= & \varphi\left[P_{1} / \operatorname{kernel}\left(P_{1} \mathbf{x}_{1}, \mathbf{q}[1]\right), \mathbf{P}_{\mathbf{2}} / \tau_{\mathbf{2}}, \ldots, P_{m} / \tau_{m}\right] \\
\psi_{2}= & \varphi\left[P_{1} / \operatorname{kernel}\left(P_{1} \mathbf{x}_{1}, \mathbf{q}[1]\right), P_{2} / \operatorname{kernel}\left(P_{2} \mathbf{x}_{2}, \mathbf{q}[2]\right), \mathbf{P}_{\mathbf{3}} / \tau_{\mathbf{3}}, \ldots, P_{m} / \tau_{m}\right] \\
& \ldots \\
\psi_{i}= & \varphi\left[P_{1} / \operatorname{kernel}\left(P_{1} \mathbf{x}_{1}, \mathbf{q}[1]\right), \ldots, P_{i} / \operatorname{kernel}\left(P_{i} \mathbf{x}_{i}, \mathbf{q}[i]\right),\right. \\
& \left.\left.\quad \mathbf{P}_{\mathbf{i}+\mathbf{1}} / \tau_{\mathbf{i}+\mathbf{1}}, \ldots P_{m} / \tau_{m}\right]\right] \\
& \ldots \\
\psi_{m}= & \varphi\left[P_{1} / \operatorname{kernel}\left(P_{1} \mathbf{x}_{1}, \mathbf{q}[1]\right), \ldots, P_{m} / \operatorname{kernel}\left(P_{m} \mathbf{x}_{m}, \mathbf{q}[m]\right)\right]=\operatorname{compress}(\varphi, \mathbf{q}) .
\end{aligned}
$$

Here, the replacement written in bold is the single predicate call to which Lemma 18 is applied. As a result, we obtain for each $0 \leq i, j \leq m$ and each pair of variables $x, y \in \operatorname{Var}(\varphi)$ that

$$
x \sim_{\psi_{i}} y \text { iff } x \sim_{\psi_{j}} y \text { and } x \in \operatorname{alloc}\left(\psi_{i}\right) \text { iff } x \in \operatorname{alloc}\left(\psi_{j}\right) .
$$

In particular, for $i=0$ and $j=m$, this yields

$$
\begin{aligned}
& x \in \operatorname{alloc}(\tau) \text { iff } x \in \operatorname{alloc}(\operatorname{compress}(\varphi, \mathbf{q})), \text { and } \\
& x \sim_{\tau} y \text { iff } x \sim_{\operatorname{compress}(\varphi, \mathbf{q})} y .
\end{aligned}
$$

It remains to prove the missing Lemmas.

Lemma 20. Given the setting of Lemma 18, it holds that

$$
\begin{aligned}
& s, h \models_{\emptyset} \varphi\left[P_{1} / \operatorname{kernel}\left(P_{1} \mathbf{x}_{1},(A, \Lambda)\right)\right] \text { implies } \\
& \exists h^{\prime} . s, h^{\prime} \models_{\emptyset} \varphi\left[P_{1} / \tau\right] \text { and } \forall y \in \operatorname{dom}(s) . \\
& \quad s(y) \in \operatorname{dom}(h) \text { iff } s(y) \in \operatorname{dom}\left(h^{\prime}\right) .
\end{aligned}
$$


Proof. Assume $s, h \models_{\emptyset} \varphi\left[P_{1} / \operatorname{kernel}\left(P_{1} \mathbf{x}_{1},(A, \Lambda)\right)\right]$. Then

$$
\begin{aligned}
& s, h \models_{\emptyset} \varphi\left[P_{1} / \operatorname{kernel}\left(P_{1} \mathbf{x}_{1},(A, \Lambda)\right)\right] \\
& \Leftrightarrow \text { [SL semantics] } \\
& \exists \mathbf{v} \in \operatorname{Val}^{\|\mathbf{z}\|} . \exists h_{1}, h_{2} . h=h_{1} \uplus h_{2} \\
& \text { and } s[\mathbf{z} \mapsto \mathbf{v}], h_{1} \models_{\emptyset} \Sigma \\
& \text { and } s[\mathbf{z} \mapsto \mathbf{v}], h_{2} \models_{\emptyset} \operatorname{kernel}\left(P_{1} \mathbf{x}_{1},(A, \Lambda)\right) \\
& \text { and } \forall a \sim b \in \Pi . s[\mathbf{z} \mapsto \mathbf{v}](a) \sim s[\mathbf{z} \mapsto \mathbf{v}](b) \\
& \Leftrightarrow \quad \text { Lemma 10] } \\
& \exists \mathbf{v} \in \operatorname{Val}^{\|\mathbf{z}\|} . \exists h_{1}, h_{2} . h=h_{1} \uplus h_{2} \\
& \text { and } s[\mathbf{z} \mapsto \mathbf{v}], h_{1} \models_{\emptyset} \Sigma \\
& \text { and }\left(s[\mathbf{z} \mapsto \mathbf{v}]\left\lceil\mathbf{x}_{0}^{\operatorname{kernel}\left(P_{1} \mathbf{x}_{1},(A, \Lambda)\right)}\right), h_{2} \models_{\emptyset} \operatorname{kernel}\left(P_{1} \mathbf{x}_{1},(A, \Lambda)\right)\right. \\
& \text { and } \forall a \sim b \in \Pi . s[\mathbf{z} \mapsto \mathbf{v}](a) \sim s[\mathbf{z} \mapsto \mathbf{v}](b) \\
& \Rightarrow \quad\left[\text { Lemma 22, } \mathbf{x}_{0}^{\operatorname{kernel}\left(P_{1} \mathbf{x}_{1},(A, \Lambda)\right)}=\mathbf{x}_{0}^{\tau}\right] \\
& \exists \mathbf{v} \in V a l^{\|\mathbf{z}\|} . \exists h_{1}, h_{2} \cdot h=h_{1} \uplus h_{2} \\
& \text { and } s[\mathbf{z} \mapsto \mathbf{v}], h_{1} \models_{\emptyset} \Sigma \\
& \text { and } \exists h_{2}^{\prime} .\left(s[\mathbf{z} \mapsto \mathbf{v}]\left\lceil\mathbf{x}_{0}^{\tau}\right), h_{2}^{\prime} \models_{\emptyset} \tau\right. \\
& \text { and } \forall y \in \operatorname{dom}\left(s[\mathbf{z} \mapsto \mathbf{v}]\left\lceil\mathbf{x}_{0}^{\tau}\right)\right. \text {. } \\
& s(y) \in \operatorname{dom}\left(h_{2}\right) \text { iff } s(y) \in \operatorname{dom}\left(h_{2}^{\prime}\right) \\
& \text { and } \forall a \sim b \in \Pi . s[\mathbf{z} \mapsto \mathbf{v}](a) \sim s[\mathbf{z} \mapsto \mathbf{v}](b) \\
& \Rightarrow\left[\text { SL semantics, } \operatorname { d o m } ( h _ { 2 } ) \subseteq \left(s[\mathbf{z} \mapsto \mathbf{v}]\left\lceil\mathbf{x}_{0}^{\tau}\right)\right.\right. \text {, } \\
& \text { set } \left.h^{\prime}=h_{1} \uplus h_{2}^{\prime}\right] \\
& \exists h^{\prime} . s, h^{\prime} \models_{\emptyset} \varphi\left[P_{1} / \tau\right] \text { and } \forall y \in \operatorname{dom}(s) \text {. } \\
& s(y) \in \operatorname{dom}(h) \text { iff } s(y) \in \operatorname{dom}\left(h^{\prime}\right) .
\end{aligned}
$$

Lemma 21. Let $\tau \in \mathrm{RSH}, A=\left\{x \in \mathbf{x}_{0} \mid x^{\tau} \in \operatorname{alloc}(\tau)\right\}$ and $\Lambda=\{x \sim$ $\left.y \mid x^{\tau} \sim_{\tau} y^{\tau}\right\}$. Moreover, let kernel $\left(P_{1} \mathbf{x}_{1},(A, \Lambda)\right)$ be defined as in Section 4. Then, for each $(s, h) \in$ States with $\operatorname{dom}(s)=\mathbf{x}_{0}^{\tau}, s, h \models_{\emptyset} \operatorname{kernel}\left(P_{1} \mathbf{x}_{1},(A, \Lambda)\right)$ holds if and only if ${ }^{14}$

$$
\begin{aligned}
& h=\{s(x) \mapsto \text { null } \mid x \in A\} \text { and } \bigwedge_{x \sim y \in \Lambda} s(x) \sim s(y) \\
& \text { and } \bigwedge_{x \in A, y \in(A \backslash\{x\}) \cup\{\text { null }\}} s(x) \neq s(y) .
\end{aligned}
$$

$\overline{14 \text { to be strict: }} h=\{\ldots\}$ is a shortcut for $\operatorname{dom}(h)=\{s(x) \mid x \in A\}, h(s(x))=$ null for each $x \in A$ 
Proof. Follows immediately from the SL semantics (cf. Figure 1).

Lemma 22. Let $\tau \in \mathrm{RSH}, A=\left\{x \in \mathbf{x}_{0} \mid x^{\tau} \in \operatorname{alloc}(\tau)\right\}$ and $\Lambda=\{x \sim$ $\left.y \mid x^{\tau} \sim_{\tau} y^{\tau}\right\}$. Moreover, let kernel $\left(P_{1} \mathbf{x}_{1},(A, \Lambda)\right)$ be defined as in Section 4. Then, for each $(s, h) \in$ States with $\operatorname{dom}(s)=\mathbf{x}_{0}^{\tau}$, we have

$$
s, h \models_{\emptyset} \operatorname{kernel}\left(P_{1} \mathbf{x}_{1},(A, \Lambda)\right) \quad \text { implies } \exists h^{\prime} . s, h^{\prime} \models_{\emptyset} \tau .
$$

Furthermore, for each $x \in \operatorname{dom}(s)$, we have $s(x) \in \operatorname{dom}(h)$ iff $s(x) \in \operatorname{dom}\left(h^{\prime}\right)$.

Proof. By structural induction on $\tau$. For readability, we omit equalities of the form $x=x$ in pure formulas although they are contained in pure formulas of $\operatorname{kernel}\left(P_{1} \mathbf{x}_{1},(A, \Lambda)\right)$ by definition.

The case $\tau=a \sim b$ is trivial, because $\operatorname{kernel}\left(P_{1} \mathbf{x}_{1},(A, \Lambda)\right)$ contains $a \sim b$.

The case $\tau=\operatorname{emp}$ is trivial, because $A=\Lambda=\emptyset$ and $\operatorname{kernel}\left(P_{1} \mathbf{x}_{1},(A, \Lambda)\right)=\operatorname{emp}$.

The case $\tau=x \mapsto(\mathbf{y})$ Then $A=\{x\}, \Lambda=\{x \neq \mathbf{n u l l}\}$. Moreover, we know that $\operatorname{kernel}\left(P_{1} \mathbf{x}_{1},(A, \Lambda)\right)=x \mapsto($ null $):\{x \neq$ null $\}$. Hence,

$$
\begin{aligned}
& s, h \models_{\emptyset} \operatorname{kernel}\left(P_{1} \mathbf{x}_{1},(A, \Lambda)\right) \\
\Rightarrow \quad & {\left[\text { Definition of } \operatorname{kernel}\left(P_{1} \mathbf{x}_{1},(A, \Lambda)\right)\right] } \\
& s, h \models_{\emptyset} x \mapsto(\mathbf{n u l l}):\{x \neq \mathbf{n u l l}\} \\
\Rightarrow \quad & {[\text { SL semantics }] } \\
& s(x) \neq \text { null and } h=\{s(x) \mapsto \text { null }\} \\
\Rightarrow \quad & {\left[\text { choose } h^{\prime}=\{s(x) \mapsto s(\mathbf{y})\}\right] } \\
& s(x) \neq \text { null and } \operatorname{dom}\left(h^{\prime}\right)=\{s(x)\} \text { and } h(s(x))=s(\mathbf{y}) \\
\Rightarrow \quad & {[\text { SL semantics }] } \\
& s, h^{\prime} \models \emptyset x \mapsto(\mathbf{y}) .
\end{aligned}
$$

Moreover, by our choice of $h^{\prime}$, we have $\operatorname{dom}(h)=\operatorname{dom}\left(h^{\prime}\right)$.

The case $\tau=\Sigma_{1} * \Sigma_{2}$ Let $A_{1}, A_{2}$ and $\Lambda_{1}, \Lambda_{2}$ denote the sets corresponding to $\Sigma_{1}$ and $\Sigma_{2}$, respectively.

$$
\begin{aligned}
& s, h \models_{\emptyset} \operatorname{kernel}\left(P_{1} \mathbf{x}_{1},(A, \Lambda)\right) \\
\Rightarrow & {\left[\text { Definition } \operatorname{kernel}\left(P_{1} \mathbf{x}_{1},(A, \Lambda)\right)\right] } \\
& s, h \models_{\emptyset} \operatorname{kernel}\left(P_{1} \mathbf{x}_{1},(A, \Lambda)\right) \text { and } h=\{s(x) \mapsto \text { null } \mid x \in A\} \\
\Leftrightarrow & {[\text { Lemma 21] }} \\
& \bigwedge_{x \sim y \in \Lambda} s(x) \sim s(y) \wedge \bigwedge_{x \in A, y \in(A \backslash\{x\}) \cup\{\text { null }\}} s(x) \neq s(y) \\
& \quad \text { and } h=\{s(x) \mapsto \text { null } \mid x \in A\} \\
\Rightarrow & {\left[A=A_{1} \uplus A_{2}\right] }
\end{aligned}
$$




$$
\begin{aligned}
& \bigwedge_{x \sim y \in \Lambda} s(x) \sim s(y) \wedge \bigwedge_{x \in A, y \in(A \backslash\{x\}) \cup\{\text { null }\}} s(x) \neq s(y) \\
& \wedge h=\left\{s(x) \mapsto \text { null } \mid x \in A_{1}\right\} \uplus\left\{s(x) \mapsto \text { null } \mid x \in A_{1}\right\} \\
& \Rightarrow \quad\left[\text { for } i=1,2 \text { choose } h_{i}=\left\{s(x) \mapsto \text { null } \mid x \mapsto(\mathbf{y}) \in \Sigma_{i}\right\}\right] \\
& \bigwedge_{x \sim y \in \Lambda} s(x) \sim s(y) \wedge \bigwedge_{x \in A, y \in(A \backslash\{x\}) \cup\{\text { null }\}} s(x) \neq s(y) \\
& \wedge h=h_{1} \uplus h_{2} \\
& \Rightarrow\left[\Lambda_{1}, \Lambda_{2} \subseteq \Lambda\right] \\
& \bigwedge_{x \sim y \in \Lambda_{1}} s(x) \sim s(y) \wedge \bigwedge_{x \sim y \in \Lambda_{2}} s(x) \sim s(y) \\
& \wedge \bigwedge_{x \in A, y \in(A \backslash\{x\}) \cup\{\text { null }\}} s(x) \neq s(y) \wedge h=h_{1} \uplus h_{2} \\
& \Rightarrow \quad[\text { Lemma 21] } \\
& s, h_{1} \models_{\emptyset} \operatorname{kernel}\left(P_{1} \mathbf{x}_{1},\left(A_{1}, \Lambda_{1}\right)\right) \text { and } s, h_{2} \models \emptyset \operatorname{kernel}\left(P_{1} \mathbf{x}_{1},\left(A_{2}, \Lambda_{2}\right)\right) \\
& \wedge \bigwedge_{x \in A, y \in(A \backslash\{x\}) \cup\{\text { null }\}} s(x) \neq s(y) \wedge h=h_{1} \uplus h_{2} \\
& \Rightarrow \text { [I.H. }] \\
& \exists h_{1}^{\prime}, h_{2}^{\prime} . s, h_{1}^{\prime} \models_{\emptyset} \Sigma_{1} \text { and } s, h_{2}^{\prime} \models_{\emptyset} \Sigma_{2} \\
& \wedge \forall x \in \operatorname{dom}(s) . s(x) \in \operatorname{dom}\left(h_{1}\right) \text { iff } s(x) \in \operatorname{dom}\left(h_{1}^{\prime}\right) \\
& \wedge \forall x \in \operatorname{dom}(s) . s(x) \in \operatorname{dom}\left(h_{2}\right) \text { iff } s(x) \in \operatorname{dom}\left(h_{2}^{\prime}\right) \\
& \wedge \bigwedge_{x \in A, y \in(A \backslash\{x\}) \cup\{\text { null }\}} s(x) \neq s(y) \wedge h=h_{1} \uplus h_{2} \\
& \Rightarrow \quad\left[\operatorname{dom}\left(h_{1}\right) \cap \operatorname{dom}\left(h_{2}\right)=\emptyset, h^{\prime}=h_{1}^{\prime} \uplus h_{2}^{\prime}\right] \\
& \exists h^{\prime}=h_{1}^{\prime} \uplus h_{2}^{\prime} . s, h_{1}^{\prime} \models_{\emptyset} \Sigma_{1} \text { and } s, h_{2}^{\prime} \models_{\emptyset} \Sigma_{2} \\
& \text { and } \forall x \in \operatorname{dom}(s) . s(x) \in \operatorname{dom}(h) \text { iff } s(x) \in \operatorname{dom}\left(h^{\prime}\right) \\
& \Rightarrow \quad \text { SL semantics] } \\
& s, h^{\prime} \models \emptyset \Sigma_{1} * \Sigma_{2} \\
& \text { and } \forall x \in \operatorname{dom}(s) . s(x) \in \operatorname{dom}(h) \text { iff } s(x) \in \operatorname{dom}\left(h^{\prime}\right)
\end{aligned}
$$

The case $\tau=\exists z . \Sigma: \Pi$ Let $\tau^{\prime}=\operatorname{strip}(\tau)=\Sigma: \Pi$ be as $\tau$ except that $z$ is a free variable. Moreover, let $A^{\prime}$ and $\Lambda^{\prime}$ be the corresponding sets of allocated variables and pure formulas between free variables of $\tau^{\prime}$. Then

$$
\begin{aligned}
& s, h \models \emptyset \operatorname{kernel}\left(P_{1} \mathbf{x}_{1},(A, \Lambda)\right) \\
& \Rightarrow \quad {[\text { Lemma 21] }} \\
& h=\{s(x) \mapsto \text { null } \mid x \in A\} \text { and } \\
& \bigwedge_{x \sim y \in \Lambda} s(x) \sim s(y) \wedge \\
& \bigwedge_{x \in A, y \in(A \backslash\{x\}) \cup\{\text { null }\}} s(x) \neq s(y)
\end{aligned}
$$




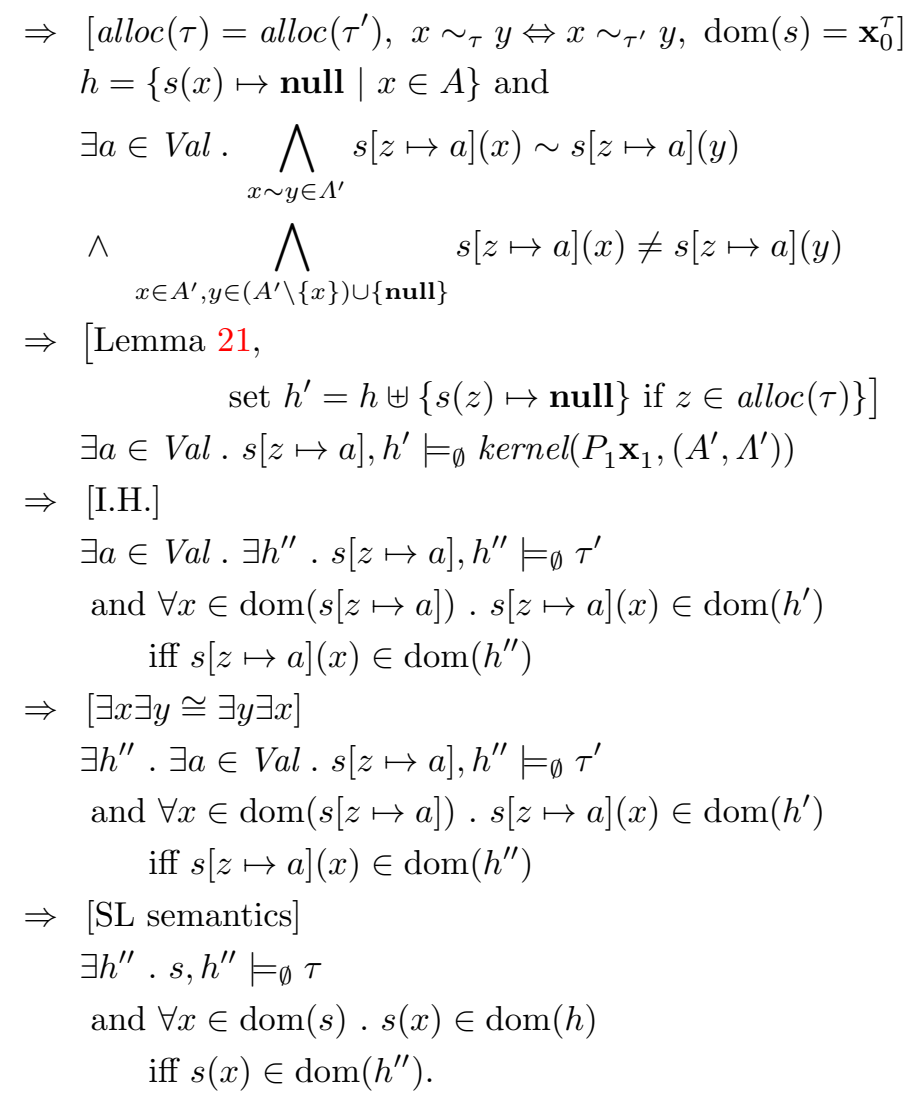

We also need the (simpler) converse direction.

Lemma 23. Let $\tau \in \mathrm{RSH}, A=\left\{x \in \mathbf{x}_{0} \mid x^{\tau} \in \operatorname{alloc}(\tau)\right\}$ and $\Lambda=\{x \sim$ $\left.y \mid x^{\tau} \sim_{\tau} y^{\tau}\right\}$. Moreover, let kernel $\left(P_{1} \mathbf{x}_{1},(A, \Lambda)\right)$ be defined as in Section 4. Then, for each $(s, h) \in$ States with $\operatorname{dom}(s)=\mathbf{x}_{0}^{\tau}$, we have

$$
s, h \models_{\emptyset} \tau \quad \text { implies } \quad \exists h^{\prime} . s, h^{\prime} \models_{\emptyset} \operatorname{kernel}\left(P_{1} \mathbf{x}_{1},(A, \Lambda)\right) .
$$

Furthermore, for each $x \in \operatorname{dom}(s)$, we have $s(x) \in \operatorname{dom}(h)$ iff $s(x) \in \operatorname{dom}\left(h^{\prime}\right)$.

Proof. Assume $s, h \models_{\emptyset} \tau$ and $\operatorname{dom}(s)=\mathbf{x}_{0}^{\tau}$. By definition of $\sim_{\tau}$ we know that for each $x, y \in \operatorname{dom}(s)$ with $x \sim_{\tau} y$, we have $s(x) \sim s(y)$. Moreover, by definition of alloc $(\tau), s(x) \in \operatorname{dom}(h)$ iff $x \in \operatorname{dom}(s) \cap \operatorname{alloc}(\tau)$. By definition of $A$ and $\Lambda$ this means

$$
\bigwedge_{x \sim y \in \Lambda} s(x) \sim s(y) \wedge \bigwedge_{x \in A, y \in(A \backslash\{x\}) \cup\{\text { null }\}} s(x) \neq s(y) .
$$

Thus, by Lemma 21, we obtain

$$
s,\{s(x) \mapsto \operatorname{null} \mid x \in A\} \models_{\emptyset} \operatorname{kernel}\left(P_{1} \mathbf{x}_{1},(A, \Lambda)\right) .
$$




\section{A.10 Proof of Theorem 3}

A heap automaton $\mathfrak{A}_{\text {SAT }}$ accepting $\operatorname{SAT}(\alpha)$ is constructed as presented in Definition 10 except for the set of final states being set to $F_{\mathfrak{A}_{\mathrm{SAT}}} \triangleq\{(A, \Pi) \mid$ null $\neq$ null $\notin \Pi\}$. Since we already now that $\mathfrak{A}_{\text {TRACK }}$ and thus also $\mathfrak{A}_{\mathrm{SAT}}$ satisfies the compositionality property (cf. Lemma 17 ), it suffices to show $L\left(\mathfrak{A}_{\mathrm{SAT}}\right)=\operatorname{SAT}(\alpha)$. Let $\tau \in \mathrm{RSH}_{\mathrm{FV} \leq \alpha}$. Then

$$
\begin{aligned}
& \tau \text { satisfiable } \\
& \Leftrightarrow \quad \text { [Definition of satisfiability] } \\
& \exists(s, h) . s, h \models_{\emptyset} \tau \\
& \Leftrightarrow \quad \text { Lemma 10] } \\
& \operatorname{Models}(\tau) \neq \emptyset \\
& \Leftrightarrow[(\forall(s, h) \in \operatorname{Models}(\tau) . s(\text { null }) \neq s(\text { null })) \\
& \text { iff } \operatorname{Models}(\tau)=\emptyset] \\
& \exists \Pi \text {. } \forall x, y \in \mathbf{x}_{0}^{\tau} \text {. null } \neq \text { null } \notin \Pi \\
& \text { and }(x \sim y) \in \Pi \\
& \leftrightarrow\left(\forall(s, h) \in \operatorname{Models}(\tau) . s\left(x^{\tau}\right) \sim s\left(y^{\tau}\right)\right) \\
& \left.\Leftrightarrow \quad \text { [Definition } x \sim_{\tau} y\right] \\
& \exists \Pi \text {. } \forall x, y \in \mathbf{x}_{0}^{\tau} \text {. null } \neq \text { null } \notin \Pi \\
& \text { and }(x \sim y) \in \Pi \leftrightarrow x^{\tau} \sim_{\tau} y^{\tau} \\
& \left.\Leftrightarrow \quad \text { [Definition of } F_{\mathfrak{A}_{\mathrm{SAT}}}\right] \\
& \exists(A, \Pi) \in F_{\mathfrak{A}_{\mathrm{SAT}}} . \forall x, y \in \mathbf{x}_{0}^{\tau} . x \in A \leftrightarrow x^{\tau} \in \operatorname{alloc}(\tau) \\
& \text { and }(x \sim y) \in \Pi \leftrightarrow x^{\tau} \sim_{\tau} y^{\tau} \\
& \Leftrightarrow \quad[\text { Definition } 10: \operatorname{compress}(\tau, \varepsilon)=\tau] \\
& \exists(A, \Pi) \in F_{\mathfrak{A}_{\mathrm{SAT}}} . \forall x, y \in \mathbf{x}_{0}^{\operatorname{compress}(\tau, \varepsilon)} . \\
& x \in A \leftrightarrow x^{\tau} \in \operatorname{alloc}(\operatorname{compress}(\tau, \varepsilon)) \\
& \text { and }(x \sim y) \in \Pi \leftrightarrow x^{\tau} \sim_{\operatorname{compress}(\tau, \varepsilon)} y^{\tau} \\
& \Leftrightarrow \quad\left[\text { Definition } \Delta_{\mathfrak{A}_{\mathrm{SAT}}}\right] \\
& \exists(A, \Pi) \in F \cdot \varepsilon \stackrel{\tau}{\rightarrow}_{\mathfrak{A}_{\mathrm{SAT}}}(A, \Pi) \\
& \left.\Leftrightarrow \quad \text { [Definition } L\left(\mathfrak{A}_{\mathrm{SAT}}\right)\right] \\
& \tau \in L\left(\mathfrak{A}_{\mathrm{SAT}}\right) \text {. }
\end{aligned}
$$

Thus, $\tau$ is satisfiable if and only if $\tau \in L\left(\mathfrak{A}_{\mathrm{SAT}}\right)$.

\section{A.11 Satisfiability is in NP}

Brotherston et al. [11] already showed that the satisfiability problem is decidable in NP if the maximal number of free variables $\alpha$ is bounded. In this section we 
briefly show that such an NP-decision procedure naturally emerged from our heap automaton $\mathfrak{A}_{\mathrm{SAT}}$ accepting $\operatorname{SAT}(\alpha)$ (see Theorem 3).

Lemma 24. SL-SAT is in NP if the maximal number $\alpha$ of free variables is bounded.

Proof. Let $(\Phi, \varphi) \in$ SL-SAT and $N=\|\Phi\|+\|\varphi\|$. Moreover, let $n \leq N$ be the maximal number of predicate calls occurring in $\varphi$ and any rule of $\Phi$. Since $\alpha$ is a constant, the number of states of heap automaton $\mathfrak{A}_{\mathrm{SAT}}$ (cf. the proof of Theorem 3) is a constant, namely $k=2^{2 \alpha^{2}+\alpha}$. Now, let $\mathbb{T}_{\Phi}(\varphi) \leq k$ denote the set of all unfolding trees $t \in \mathbb{T}_{\Phi}(\varphi)$ of height at most $k$. Clearly, each of these trees is of size $\|t\| \leq n^{k} \leq N^{k}$, i.e., polynomial in $N$. Moreover, let $\omega: \operatorname{dom}(t) \rightarrow Q_{\mathfrak{A}_{\mathrm{SAT}}}$ be a function mapping each node of $t$ to a state of $\mathfrak{A}_{\mathrm{SAT}}$. Again, $\omega$ is of size polynomial in $N$; as such $\|\omega\| \leq k \cdot N^{k}$. Let $\Omega_{t}$ denote the set of all of these functions $\omega$ for a given unfolding tree $t$ with $\omega(\varepsilon) \in F_{\mathfrak{A}_{\mathrm{SAT}}}$. Now, given an unfolding tree $t \in \mathbb{T}_{\Phi}(\varphi) \leq k$ and $\omega \in \Omega_{t}$, we can easily decide whether $\varepsilon \stackrel{\llbracket t \rrbracket}{\longrightarrow}_{\mathfrak{A}} \omega(\varepsilon)$ holds: For each $u, u 1, \ldots, u n \in \operatorname{dom}(t), u(n+1) \notin \operatorname{dom}(t), n \geq 0$ it suffices to check whether $\omega(u 1) \ldots \omega(u n) \stackrel{t(u)}{\longrightarrow} \mathfrak{A} \omega(u)$. Since, by Remark 2, each of these checks can be performed in time polynomial in $N$, the whole procedure is feasible in polynomial time.

Then, our decision procedure for SL-SAT answers yes on input $(\Phi, \varphi)$ if and only if

$$
\exists t \in \mathbb{T}_{\Phi}(\varphi)^{\leq k} . \forall \omega \in \Omega_{t} . \operatorname{not} \varepsilon \stackrel{\llbracket t \rrbracket}{\longrightarrow} \mathfrak{A} \omega(\varepsilon) .
$$

Since $t$ and $\omega$ are both of size polynomial in $N$, this procedure is in NP. Regarding correctness, we first note that $\mathbb{U}_{\Phi}(\varphi) \cap \operatorname{SAT}(\alpha) \neq \emptyset$ holds iff $\llbracket t \rrbracket \in \operatorname{SAT}(\alpha)$ for some $t \in \mathbb{T}_{\Phi}(\varphi)$. Furthermore, by a standard pumping argument, it suffices to consider trees in $\mathbb{T}_{\Phi}(\varphi)^{\leq k}$ : If there exists a taller tree $t$ with $\llbracket t \rrbracket \in \operatorname{SAT}(\alpha)$ then there is some path of length greater $k$ in $t$ on which two nodes are assigned the same state by a function $\omega \in \Omega_{t}$ proving membership of $t$ in $\operatorname{SAT}(\alpha)$. Thus, this path can be shortened to obtain a tree of smaller height whose unfolding is satisfiable.

\section{A.12 Compositionality of $\mathfrak{A}_{\mathrm{EST}}, \mathfrak{A}_{\mathrm{GFREE}}, \mathfrak{A}_{\mathrm{ACYCLIC}}$}

Some of our heap automata presented in Section 4 follow a common construction scheme. In particular this holds for

- $\mathfrak{A}_{\mathrm{EST}}$ (cf. Lemma 4 and Appendix A.13),

- $\mathfrak{A}_{\mathrm{GFREE}}$ (cf. Lemma 6 and Appendix A.17), and

- $\mathfrak{A}_{\text {ACYCLIC }}$ (cf. Lemma 7 and Appendix A.19).

Intuitively, each of these automata evaluates a predicate check : $\mathrm{SH}_{\mathrm{FV} \leq \alpha} \times Q_{\mathfrak{A}}^{*} \rightarrow$ $\{0,1\}$ while running a heap automaton $\mathfrak{A}$ - which is either $\mathfrak{A}_{\text {TRACK }}$ (cf. Definition 10) or $\mathfrak{A}_{\text {REACH }}$ (cf. Lemma 6) - in parallel to collect required knowledge about the relationships (equalities, allocation, reachability) between free variables. 
Due to these similarities, we prove the compositionality property for a general construction scheme as described above. More formally,

Definition 17. Let $\mathfrak{A} \in\left\{\mathfrak{A}_{\mathrm{SAT}}, \mathfrak{A}_{\mathrm{REACH}}\right\}$ be either the tracking automaton $\mathfrak{A}_{\mathrm{TRACK}}$ (cf. Definition 10) or the reachability automaton $\mathfrak{A}_{\mathrm{REACH}}$ (cf. Lemma 6). Moreover, let $F \subseteq Q_{\mathfrak{A}} \times\{0,1\}$. Further, let check $: \mathrm{SH}_{F V \leq \alpha} \times Q_{\mathfrak{A}}^{\star} \rightarrow\{0,1\}$ be a Boolean predicate such that for each $\varphi \in \mathrm{SH}_{F V \leq \alpha}$ with $\left\|\Gamma^{\varphi}\right\|=m \tau_{1}, \ldots, \tau_{m} \in$ $\mathrm{RSH}_{F V \leq \alpha}, p_{1} \ldots p_{m} \stackrel{\varphi}{\rightarrow} \mathfrak{A} p_{0}$, and $\varepsilon \stackrel{\tau_{i}}{\rightarrow} \mathfrak{A} p_{i}$, we have

$$
\operatorname{check}\left(\varphi\left[P_{1}^{\varphi} / \tau_{1}, \ldots, P_{m}^{\varphi} / \tau_{m}\right], \varepsilon\right)=1
$$

if and only if such that

$$
\operatorname{check}\left(\varphi, p_{1} \ldots p_{m}\right)=\operatorname{check}\left(\tau_{1}, \varepsilon\right)=\ldots=\operatorname{check}\left(\tau_{m}, \varepsilon\right)=1 .
$$

Then the heap automaton $\mathfrak{A}_{\text {SCHEME }}(\mathfrak{A}$, check, $F)$ is given by

$$
\begin{aligned}
& \mathfrak{A}_{\text {SCHEME }}(\mathfrak{A}, \text { check }, F)=\left(Q, F V^{\leq \alpha}, \Delta, F\right), \text { where } \\
& Q \triangleq Q_{\mathfrak{A}} \times\{0,1\} \\
& \Delta \quad: \quad\left(p_{1}, q_{1}\right) \ldots\left(p_{m}, q_{m}\right) \stackrel{\varphi}{\rightarrow} \mathfrak{B}\left(p_{0}, q_{0}\right) \\
& \text { iff } p_{1} \ldots p_{m} \stackrel{\varphi}{\rightarrow}{ }_{\mathfrak{A}} p_{0} \\
& \quad \text { and } q_{0}=\min \left\{q_{1}, \ldots, q_{m}, \operatorname{check}\left(\varphi, p_{1} \ldots p_{m}\right)\right\},
\end{aligned}
$$

where $\left\|\Gamma^{\varphi}\right\|=m \geq 0$.

Lemma 25. The heap automaton $\mathfrak{B}=\mathfrak{A}_{\text {SCHEME }}(\mathfrak{A}$, check, $F)$ satisfies the compositionality property.

Proof. Let $\varphi \in \mathrm{SH}_{\mathrm{FV} \leq \alpha}$ with $\left\|\Gamma^{\varphi}\right\|=m$ and $\tau_{1}, \ldots, \tau_{m} \in \mathrm{RSH}_{\mathrm{FV} \leq \alpha}$. Moreover, let $\tau=\varphi\left[P_{1}^{\varphi} / \tau_{1}, \ldots, P_{m}^{\varphi} / \tau_{m}\right]$. We have to show that for each $\left(p_{0}, q_{0}\right) \in Q_{\mathfrak{B}}$ it holds that

$$
\begin{aligned}
\varepsilon \stackrel{\tau}{\rightarrow}_{\mathfrak{B}}\left(p_{0}, q_{0}\right) \quad \text { iff } \quad & \exists\left(p_{1}, q_{1}\right), \ldots,\left(p_{m}, q_{m}\right) \in Q_{\mathfrak{B}} . \\
& \left(p_{1}, q_{1}\right) \ldots\left(p_{m}, q_{m}\right) \stackrel{\varphi}{\rightarrow}_{\mathfrak{B}}\left(p_{0}, q_{0}\right) \\
& \text { and } \forall 1 \leq i \leq m \cdot \varepsilon{\stackrel{\tau_{i}}{\rightarrow}}_{\mathfrak{B}}\left(p_{i}, q_{i}\right) .
\end{aligned}
$$

Assume $\varepsilon \stackrel{\tau}{\rightarrow}{ }_{\mathfrak{B}}\left(p_{0}, q_{0}\right)$. By definition of $\Delta_{\mathfrak{B}}$ this is the case if and only if $\varepsilon \stackrel{\tau}{\rightarrow} \mathfrak{A} p_{0}$ and $q_{0}=\operatorname{check}(\tau, \varepsilon)$. For each $1 \leq i \leq m$, we set $q_{i}=\operatorname{check}\left(\tau_{i}, \varepsilon\right) \in$ $\{0,1\}$. Moreover, since $\mathfrak{A}$ is known to satisfy the compositionality property by Lemma 3 (or Lemma 6), this is equivalent to

$$
\begin{aligned}
& \qquad q_{0}=\operatorname{check}(\tau, \varepsilon) \text { and } \forall 1 \leq i \leq m \cdot q_{i}=\operatorname{check}\left(\tau_{i}, \varepsilon\right) \\
& \text { and } \exists p_{1}, \ldots, p_{m} \in Q_{\mathfrak{A}} \cdot p_{1} \ldots p_{m} \stackrel{\varphi}{\rightarrow} \mathfrak{A} p_{0} \\
& \text { and } \forall 1 \leq i \leq m . \varepsilon \stackrel{\tau_{i}}{\rightarrow} p_{\mathfrak{A}} p_{0} .
\end{aligned}
$$


By construction of $\mathfrak{B}$ and since each $\tau_{i}$ contains no predicate calls this is equivalent to

$$
\begin{aligned}
& \qquad q_{0}=\operatorname{check}(\tau, \varepsilon) \\
& \text { and } \exists\left(p_{1}, q_{1}\right), \ldots,\left(p_{m}, q_{m}\right) \in Q_{\mathfrak{B}} \cdot p_{1} \ldots p_{m} \stackrel{\varphi}{\rightarrow}_{\mathfrak{A}} p_{0} \\
& \text { and } \forall 1 \leq i \leq m \cdot \varepsilon{\stackrel{\tau_{i}}{\rightarrow}}_{\mathfrak{B}}\left(p_{0}, q_{0}\right) .
\end{aligned}
$$

Now, by Definition $17, \operatorname{check}(\tau, \varepsilon)=1$ if and only if $\operatorname{check}\left(\varphi, p_{1} \ldots p_{m}\right)=1$ and $\operatorname{check}\left(\tau_{1}, \varepsilon\right)=\ldots=\operatorname{check}\left(\tau_{m}, \varepsilon\right)=1$. Thus

$$
\begin{aligned}
q_{0}=\operatorname{check}(\tau, \varepsilon)= & \min \left\{\operatorname{check}\left(\varphi, p_{1} \ldots p_{m}\right)\right. \\
& \left.\operatorname{check}\left(\tau_{1}, \varepsilon\right), \ldots \operatorname{check}\left(\tau_{m}, \varepsilon\right)\right\} \\
= & \min \left\{\operatorname{check}\left(\varphi, p_{1} \ldots p_{m}\right), q_{1}, \ldots, q_{m}\right\} .
\end{aligned}
$$

Putting this equation into $(\dagger)$, we obtain the equivalent statement

$$
\begin{aligned}
& \quad \exists\left(p_{1}, q_{1}\right), \ldots,\left(p_{m}, q_{m}\right) \in Q_{\mathfrak{B}} \cdot p_{1} \ldots p_{m} \stackrel{\varphi}{\rightarrow} \mathfrak{A} p_{0} \\
& \text { and } q_{0}=\min \left\{\operatorname{check}\left(\varphi, p_{1} \ldots p_{m}\right), q_{1}, \ldots, q_{m}\right\} \\
& \text { and } \forall 1 \leq i \leq m \cdot \varepsilon \stackrel{\tau_{i}}{\rightarrow} \mathfrak{B}\left(p_{0}, q_{0}\right) .
\end{aligned}
$$

By definition of $\Delta_{\mathfrak{B}}$, this is equivalent to

$$
\begin{aligned}
& \exists\left(p_{1}, q_{1}\right), \ldots,\left(p_{m}, q_{m}\right) \in Q_{\mathfrak{B}} . \\
& \quad\left(p_{1}, q_{1}\right) \ldots\left(p_{m}, q_{m}\right) \stackrel{\varphi}{\rightarrow}_{\mathfrak{B}}\left(p_{0}, q_{0}\right) \\
& \text { and } \forall 1 \leq i \leq m . \varepsilon{\stackrel{\tau_{i}}{\rightarrow}}_{\mathfrak{B}}\left(p_{0}, q_{0}\right) .
\end{aligned}
$$

Hence, $\mathfrak{B}=\mathfrak{A}_{\mathrm{SCHEME}}(\mathfrak{A}$, check,$F)$ satisfies the compositionality property.

\section{A.13 Proof of Theorem 4}

We have to construct a heap automaton $\mathfrak{A}_{\mathrm{EST}}$ over $\mathrm{SH}_{\mathrm{FV} \leq \alpha}$ that satisfies the compositionality property and accepts $\operatorname{EST}(\alpha)$. In order to highlight the necessary proof obligations, the actual construction of $\mathfrak{A}_{\mathrm{EST}}$ and its correctness proof are splitted into several definitions and lemmas that are provided afterwards.

The construction of $\mathfrak{A}_{\mathrm{EST}}$ was already sketched in the paper. A fully formal construction of $\mathfrak{A}_{\mathrm{EST}}$ is found in Definition 18. It then remains to show the correctness of our construction of $\mathfrak{A}_{\mathrm{EST}}$ :

- Lemma 26 establishes that $\mathfrak{A}_{\mathrm{EST}}$ indeed accepts $\operatorname{EST}(\alpha)$, i.e., $L\left(\mathfrak{A}_{\mathrm{EST}}\right)=$ $\operatorname{EST}(\alpha)$.

- To prove the compositionality property, we show that $\mathfrak{A}_{\mathrm{EST}}$ is an instance of a more general construction scheme whose compositionality property is shown in Lemma 25. In order to apply Lemma 25, we have to show that

$$
\operatorname{check}\left(\varphi\left[P_{1}^{\varphi} / \tau_{1}, \ldots, P_{m}^{\varphi} / \tau_{m}\right], \varepsilon\right)=1
$$




$$
\begin{aligned}
& \text { iff } \operatorname{check}\left(\varphi, p_{1} \ldots p_{m}\right)=1 \\
& \quad \text { and } \operatorname{check}\left(\tau_{1}, \varepsilon\right)=\ldots=\operatorname{check}\left(\tau_{m}, \varepsilon\right)=1 .
\end{aligned}
$$

This is verified in Lemma 27. Then, by Lemma 25 , we know that $\mathfrak{A}_{\mathrm{EST}}=$ $\mathfrak{A}_{\text {SCHEME }}\left(\mathfrak{A}_{\text {TRACK }}\right.$, check,$\left.F\right)$ satisfies the compositionality property.

Putting both together, we obtain a heap automaton $\mathfrak{A}_{\mathrm{EST}}$ over $\mathrm{SH}_{\mathrm{FV} \leq \alpha}$ that satisfies the compositionality property and accepts $\operatorname{EST}(\alpha)$.

The remainder of this section fills the gaps in the proof from above.

Definition 18. $\mathfrak{A}_{\mathrm{EST}}=\left(Q, \mathrm{SH}_{F V \leq \alpha}, \Delta, F\right)$ is given by

$$
\begin{aligned}
& Q \triangleq Q_{\mathfrak{A}_{\mathrm{TRACK}}} \times\{0,1\}, \quad F \triangleq Q_{\mathfrak{A}_{\mathrm{TRCK}}} \times\{1\}, \\
& \Delta: \quad\left(p_{1}, q_{1}\right) \ldots\left(p_{m}, q_{m}\right) \stackrel{\varphi}{\rightarrow}_{\mathfrak{A}_{\mathrm{EST}}}\left(p_{0}, q_{0}\right) \\
& \quad \text { iff } p_{1} \ldots p_{m} \stackrel{\varphi}{\rightarrow}_{\mathfrak{A}_{\mathrm{TRACK}}} p_{0} \\
& \quad \text { and } q_{0}=\min \left\{q_{1}, \ldots, q_{m}, \operatorname{check}\left(\varphi, p_{1} \ldots p_{m}\right)\right\} .
\end{aligned}
$$

Here, check : $\mathrm{SH}_{F V \leq \alpha} \times Q_{\mathfrak{A}_{\text {TRACK }}}^{*} \rightarrow\{0,1\}$ is a predicate given by

$$
\operatorname{check}(\varphi, \mathbf{p}) \triangleq\left\{\begin{array}{c}
1, \quad \text { if } \forall y \in \operatorname{Var}(\varphi) \cdot y \in \operatorname{alloc}(\operatorname{compress}(\varphi, \mathbf{p})) \\
\quad \text { or } \exists x \in \mathbf{x}_{0}^{\varphi} \cdot x=\operatorname{compress}(\varphi, \mathbf{p}) y \\
0, \text { otherwise }
\end{array}\right.
$$

where compress $(\varphi, \mathbf{p})$ is the reduced symbolic heap obtained from the tracking property as in Definition 10.

Lemma 26. $L\left(\mathfrak{A}_{\mathrm{EST}}\right)=\operatorname{EST}(\alpha)$.

Proof. Let $\tau \in \mathrm{RSH}_{\mathrm{FV} \leq \alpha}$. Then:

$$
\begin{aligned}
& \tau \in L\left(\mathfrak{A}_{\mathrm{EST}}\right) \\
\Leftrightarrow & {\left[\text { Definition of } L\left(\mathfrak{A}_{\mathrm{EST}}\right)\right] } \\
& \exists q \in F_{\mathfrak{A}_{\mathrm{EST}}} \cdot \varepsilon \stackrel{\tau}{\rightarrow}_{\mathfrak{A}_{\mathrm{EST}}} q \\
\Leftrightarrow \quad & {\left[\text { Definition of } F_{\mathfrak{A}_{\mathrm{EST}}}, q=(p, 1)\right] } \\
& \exists p \in Q_{\mathfrak{A}_{\mathrm{TRACK}}} \cdot \varepsilon \stackrel{\tau}{\rightarrow}_{\mathfrak{A}_{\mathrm{EST}}}(p, 1) \\
\Leftrightarrow \quad & {\left[\text { Definition of } \Delta_{\mathfrak{A}_{\mathrm{EST}}}\right] } \\
& \exists p \in Q_{\mathfrak{T}_{\mathrm{TRACK}}} \cdot \varepsilon \stackrel{\tau}{\rightarrow}_{\mathfrak{A}_{\mathrm{TRACK}}} p \text { and } \operatorname{check}(\tau, \varepsilon)=1 \\
\Leftrightarrow \quad & {[\text { Definition of } \operatorname{check}(\tau, \varepsilon)] } \\
& \exists p \in Q_{\mathfrak{A}_{\mathrm{TRACK}}} \cdot \varepsilon \stackrel{\tau}{\rightarrow}_{\mathfrak{A}_{\mathrm{TRACK}}} p \\
& \quad \text { and } \forall y \in \operatorname{Var}(\tau) \cdot y \in \operatorname{alloc}(\operatorname{compress}(\tau, \varepsilon)) \\
& \quad \text { or } \exists x \in \mathbf{x}_{0}^{\tau} \cdot x=\operatorname{compress}(\tau, \varepsilon) \\
\Leftrightarrow & {\left[\left\|\Gamma^{\tau}\right\|=0 \text { implies } \tau=\operatorname{compress}(\tau, \varepsilon)\right] }
\end{aligned}
$$




$$
\begin{aligned}
& \forall y \in \operatorname{Var}(\tau) \cdot y \in \operatorname{alloc}(\tau) \text { or } \exists x \in \mathbf{x}_{0}^{\tau} \cdot x={ }_{\tau} y \\
\Leftrightarrow & {[\text { Definition of } \operatorname{EST}(\alpha)] } \\
& \tau \in \operatorname{EST}(\alpha) .
\end{aligned}
$$

Lemma 27. Let $\varphi \in \mathrm{SH}_{F V \leq \alpha}$ with $\left\|\Gamma^{\varphi}\right\|=m$ and $\tau_{1}, \ldots, \tau_{m} \in \mathrm{RSH}_{F V \leq \alpha}$. Then

$$
\begin{aligned}
& \operatorname{check}\left(\varphi\left[P_{1}^{\varphi} / \tau_{1}, \ldots, P_{m}^{\varphi} / \tau_{m}\right], \varepsilon\right)=1 \\
& \text { iff } \operatorname{check}(\varphi, \mathbf{p}) \\
& \quad \text { and } \operatorname{check}\left(\tau_{1}, \varepsilon\right)=\ldots=\operatorname{check}\left(\tau_{m}, \varepsilon\right)=1 .
\end{aligned}
$$

The proof of Lemma 27 relies on a technical observation, which intuitively states that equalities between variables belonging to different nodes of an unfolding tree have to be propagated through parameters. Formally,

Lemma 28. Let $\varphi \in \mathrm{SH}_{F V \leq \alpha}$ with $\left\|\Gamma^{\varphi}\right\|=m, \tau_{1}, \ldots, \tau_{m} \in \mathrm{RSH}_{F V \leq \alpha}$ and $\tau=$ $\varphi\left[P_{1}^{\varphi} / \tau_{1}, \ldots, P_{m}^{\varphi} / \tau_{m}\right]$. Moreover, for some $1 \leq i \leq m$, let $x \in \operatorname{Var}\left(\tau_{i}\left[\mathbf{x}_{0}^{\tau_{i}} / \mathbf{x}_{i}^{\varphi}\right]\right)$ and $y \in \operatorname{Var}(\tau) \backslash \operatorname{Var}\left(\tau_{i}\left[\mathbf{x}_{0}^{\tau_{i}} / \mathbf{x}_{i}^{\varphi}\right]\right)$. Then $x={ }_{\tau} y \quad$ iff $\exists z \in \mathbf{x}_{i}^{\varphi} \cdot x={ }_{\tau_{i}\left[\mathbf{x}_{0}^{\tau_{i}} / \mathbf{x}_{i}^{\varphi}\right]}$ $z$ and $z={ }_{\tau} y$.

Proof (sketch). The direction from right to left is straightforward. For the converse direction, observe that, by Lemma 15 , a pure formula $x=y$ is an element of the closure of pure formulas of $\tau$. However, such an equality cannot be an element of the closures of $\Pi^{\varphi}, \Pi^{\tau_{1}}, \ldots, \Pi^{\tau_{m}}$, because, by assumption, $x$ and $y$ are not both contained in the set of variables of these symbolic heaps. Thus, since the only variables shared by $\varphi$ and $\tau_{1}, \ldots, \tau_{m}$ are the parameters of the predicate calls of $\varphi$, there exists a parameter $z$ of a suitable predicate call such that $x=z$ is contained in one of the aforementioned closures of pure formulas.

Note that null is always assumed to be a free variable and no other constants occur in our fragment of symbolic heaps. Otherwise, the observation from above would be wrong.

Proof (of Lemma 27). Let $\tau=\varphi\left[P_{1}^{\varphi} / \tau_{1}, \ldots, P_{m}^{\varphi} / \tau_{m}\right]$. Recall that $\operatorname{compress}(\tau, \varepsilon$ ) denotes the reduced symbolic heap introduced in Definition 10. Then:

$$
\begin{aligned}
& \operatorname{check}(\tau, \varepsilon)=1 \\
\Leftrightarrow & {[\operatorname{Definition} \text { of check, compress }(\tau, \varepsilon)=\tau] } \\
& \forall y \in \operatorname{Var}(\tau) \cdot y \in \operatorname{alloc}(\tau) \text { or } \exists x \in \mathbf{x}_{0}^{\tau} \cdot x={ }_{\tau} y \\
\Leftrightarrow & {\left[\operatorname{Var}(\tau)=\operatorname{Var}(\varphi) \cup \bigcup_{1 \leq i \leq m} \operatorname{Var}\left(\tau_{i}\left[\mathbf{x}_{0}^{\tau_{i}} / \mathbf{x}_{i}^{\varphi}\right]\right)\right] } \\
& \forall y \in \operatorname{Var}(\varphi) \cdot y \in \operatorname{alloc}(\tau) \operatorname{or} \exists x \in \mathbf{x}_{0}^{\tau} \cdot x={ }_{\tau} y \\
& \text { and } \forall 1 \leq i \leq m . \forall y \in \operatorname{Var}\left(\tau_{i}\left[\mathbf{x}_{0}^{\tau_{i}} / \mathbf{x}_{i}^{\varphi}\right]\right) .
\end{aligned}
$$




$$
y \in \operatorname{alloc}(\tau) \text { or } \exists x \in \mathbf{x}_{0}^{\tau} \cdot x=_{\tau} y
$$

$\Leftrightarrow \quad$ [Lemma 19 applied to $y \in \operatorname{Var}(\varphi)$ and $\left.x \in \operatorname{Var}\left(\mathbf{x}_{0}^{\tau}\right)\right]$

$\forall y \in \operatorname{Var}(\varphi) . y \in \operatorname{alloc}(\operatorname{compress}(\varphi, \mathbf{p}))$

or $\exists x \in \mathbf{x}_{0}^{\varphi} \cdot x={ }_{\operatorname{compress}}(\varphi, \mathbf{p}) y$

and $\forall 1 \leq i \leq m . \forall y \in \operatorname{Var}\left(\tau_{i}\left[\mathbf{x}_{0}^{\tau_{i}} / \mathbf{x}_{i}^{\varphi}\right]\right)$.

$y \in \operatorname{alloc}(\tau)$ or $\exists x \in \mathbf{x}_{0}^{\tau} \cdot x={ }_{\tau} y$

$\Leftrightarrow$ [Definition of check]

$\operatorname{check}(\operatorname{compress}(\varphi, \mathbf{p}))=1$

and $\forall 1 \leq i \leq m . \forall y \in \operatorname{Var}\left(\tau_{i}\left[\mathbf{x}_{0}^{\tau_{i}} / \mathbf{x}_{i}^{\varphi}\right]\right)$.

$y \in \operatorname{alloc}(\tau)$ or $\exists x \in \mathbf{x}_{0}^{\tau} \cdot x={ }_{\tau} y$

$\Leftrightarrow\left[y \in \operatorname{alloc}(\tau)\right.$ iff $y \in \operatorname{alloc}\left(\tau_{i}\left[\mathbf{x}_{0}^{\tau_{i}} / \mathbf{x}_{i}^{\varphi}\right]\right)$

or $\left.\exists x \in \operatorname{alloc}(\tau) \cdot y={ }_{\tau} x\right]$

$\operatorname{check}(\operatorname{compress}(\varphi, \mathbf{p}))=1$

and $\forall 1 \leq i \leq m . \forall y \in \operatorname{Var}\left(\tau_{i}\left[\mathbf{x}_{0}^{\tau_{i}} / \mathbf{x}_{i}^{\varphi}\right]\right)$.

$\left(y \in \operatorname{alloc}\left(\tau_{i}\left[\mathbf{x}_{0}^{\tau_{i}} / \mathbf{x}_{i}^{\varphi}\right]\right)\right.$ or $\left.\exists x \in \operatorname{alloc}(\tau) \cdot y={ }_{\tau} x\right)$

or $\exists x \in \mathbf{x}_{0}^{\tau} \cdot x={ }_{\tau} y$

$\Leftrightarrow$ [Lemma 28]

$\operatorname{check}(\operatorname{compress}(\varphi, \mathbf{p}))=1$

and $\forall 1 \leq i \leq m . \forall y \in \operatorname{Var}\left(\tau_{i}\left[\mathbf{x}_{0}^{\tau_{i}} / \mathbf{x}_{i}^{\varphi}\right]\right)$.

$y \in \operatorname{alloc}\left(\tau_{i}\right)$

or $\exists z \in \mathbf{x}_{i}^{\varphi} \cdot \exists x \in \operatorname{alloc}(\tau) \cdot y=_{\tau_{i}} z$ and $z=_{\tau} x$

or $\exists x \in \mathbf{x}_{i}^{\varphi} \cdot \exists z \in \mathbf{x}_{0}^{\tau} \cdot y={ }_{\tau_{i}} x$ and $x={ }_{\tau} z$

$\Leftrightarrow\left[\mathbf{x}_{0}^{\tau_{i}}\right.$ is substituted by $\mathbf{x}_{i}^{\varphi} \in \operatorname{Var}(\varphi)$ in $\tau$

which are all established due to $\operatorname{check}(\operatorname{compress}(\varphi, \mathbf{p}))=1]$

$\operatorname{check}(\operatorname{compress}(\varphi, \mathbf{p}))=1$

and $\forall 1 \leq i \leq m . \forall y \in \operatorname{Var}\left(\tau_{i}\right)$.

$y \in \operatorname{alloc}\left(\tau_{i}\right)$ or $\exists z \in \mathbf{x}_{0}^{\tau_{i}} \cdot y={ }_{\tau_{i}} z$

$\Leftrightarrow$ [Definition of check]

$\operatorname{check}(\operatorname{compress}(\varphi, \mathbf{p}))=1$

and $\operatorname{check}\left(\tau_{1}, \varepsilon\right)=\ldots=\operatorname{check}\left(\tau_{m}, \varepsilon\right)=1$.

\section{A.14 Proof of Lemma 4}

Let $(\Phi, P) \in \overline{\text { SL-RSAT }}$ be an instance of the complement of the reduced satisfiability problem. Moreover, consider the instance $(\Phi, \varphi)$ of the establishment 
problem, where

$$
\varphi \triangleq \exists \mathbf{z} z^{\prime} . P \mathbf{z}:\left\{x=\text { null, } z^{\prime} \neq \mathbf{n u l l}\right\}
$$

and $x$ is the single free variable (other than null) of $\varphi$. Then

$$
\begin{aligned}
& \mathbb{U}_{\Phi}(\varphi) \subseteq \operatorname{EST}(\alpha) \\
\Leftrightarrow & {[A \subseteq B \text { iff } \forall x \in A . x \in B] } \\
& \forall \tau \in \mathbb{U}_{\Phi}(\varphi) \cdot \tau \in \operatorname{EST}(\alpha) \\
\Leftrightarrow & {[\text { Definition of } \operatorname{EST}(\alpha)] } \\
& \forall \tau \in \mathbb{U}_{\Phi}(\varphi) \cdot \forall y \in \operatorname{Var}(\tau) \cdot \\
& y \in \text { alloc }(\tau) \text { or } \exists x \in \mathbf{x}_{0}^{\tau} \cdot y={ }_{\tau} x \\
\Leftrightarrow & {[\Phi \text { contains no points-to assertions }] } \\
& \forall \tau \in \mathbb{U}_{\Phi}(\varphi) \cdot \forall y \in \operatorname{Var}(\tau) \cdot \exists x \in \mathbf{x}_{0}^{\tau} \cdot y={ }_{\tau} x \\
\Leftrightarrow & {\left[\mathbf{x}_{0}^{\tau}[1]={ }_{\tau} \text { null }\right] } \\
& \forall \tau \in \mathbb{U}_{\Phi}(\varphi) \cdot \forall y \in \operatorname{Var}(\tau) \cdot y={ }_{\tau} \text { null } \\
\Leftrightarrow & {\left[z^{\prime} \neq{ }_{\tau} \text { null }\right] } \\
& \forall \tau \in \mathbb{U}_{\Phi}(\varphi) \cdot \text { null } \neq{ }_{\tau} \text { null } \\
\Leftrightarrow & {\left[\text { null } \neq{ }_{\tau} \text { null iff } \tau \text { unsatisfiable }\right] } \\
& \forall \tau \in \mathbb{U}_{\Phi}(\varphi) \cdot \tau \text { is unsatisfiable } \\
\Leftrightarrow & {[\forall x . \neg A \equiv \neg \exists x . A] } \\
\Leftrightarrow & \text { not } \exists \tau \in \mathbb{U}_{\Phi}(\varphi) \cdot \tau \text { if satisfiable } \\
\Leftrightarrow & {[\text { Definition of satisfiability }] } \\
& \varphi \text { is unsatisfiable. }
\end{aligned}
$$

Then it remains to show that $\varphi$ is unsatisfiable if and only if $P$ is unsatisfiable:

$$
\begin{aligned}
& \exists(s, h) \in \text { States } .(s, h) \models_{\Phi} \varphi \\
\Leftrightarrow \quad & {[\text { Applying }(\mathbf{(}) \text { see below }] } \\
& \exists(s, h) \in \text { States } . \exists \mathbf{u} \in \text { Val }^{\|\mathbf{z}\|} . \\
& \quad\left(s[\mathbf{z} \mapsto \mathbf{u}] \Upsilon_{\mathbf{z}}, h\right) \models_{\Phi} P \mathbf{z} \\
& \text { and } s(x)=\text { null and } s\left(z^{\prime}\right) \neq \text { null } \\
\Leftrightarrow \quad & {\left[\operatorname{dom}\left(s[\mathbf{z} \mapsto \mathbf{z}] \Upsilon_{\mathbf{z}}\right)=\mathbf{z}\right] } \\
& \exists(s, h) \in \text { States } .(s, h) \models_{\Phi} P \mathbf{z} .
\end{aligned}
$$

Here, the missing step marked with ( $)$ corresponds to the following property:

$$
\begin{aligned}
& s, h \models_{\Phi} \varphi \\
& \Leftrightarrow \exists \mathbf{u} \in V a l^{\|\mathbf{z}\|} . \\
&\left(s[\mathbf{z} \mapsto \mathbf{u}] \Upsilon_{\mathbf{z}}\right), h \models_{\Phi} P \mathbf{z} \\
& \text { and } s(x)=\text { null and } s\left(z^{\prime}\right) \neq \text { null. }
\end{aligned}
$$


To complete the proof, let $(s, h) \in$ States. Then:

$$
\begin{aligned}
& s, h \models_{\Phi} \varphi \\
& \Leftrightarrow \text { [Construction of } \varphi] \\
& s, h \models_{\Phi} \exists \mathbf{z} z^{\prime} . P \mathbf{z}:\left\{x=\text { null, } z^{\prime} \neq \mathbf{n u l l}\right\} \\
& \Leftrightarrow \text { [SL semantics] } \\
& \exists \mathbf{u} \in \operatorname{Val}^{\|\mathbf{z}\|} . \exists v \in \text { Val . } \\
& s\left[\mathbf{z} \mapsto \mathbf{u}, z^{\prime} \mapsto v\right], h \models_{\Phi} P \mathbf{z} \\
& \text { and } s\left[\mathbf{z} \mapsto \mathbf{u}, z^{\prime} \mapsto v\right], h \models_{\Phi} x=\text { null } \\
& \text { and } s\left[\mathbf{z} \mapsto \mathbf{u}, z^{\prime} \mapsto v\right], h \models_{\Phi} z^{\prime} \neq \mathbf{n u l l} \\
& \Leftrightarrow \text { [Lemma 10] } \\
& \exists \mathbf{u} \in \operatorname{Val}^{\|\mathbf{z}\|} . \exists v \in V a l . \\
& \left(s\left[\mathbf{z} \mapsto \mathbf{u}, z^{\prime} \mapsto v\right] \Upsilon_{\mathbf{z}}\right), h \models_{\Phi} P \mathbf{z} \\
& \text { and }\left(s\left[\mathbf{z} \mapsto \mathbf{u}, z^{\prime} \mapsto v\right]\left\lceil_{x}\right), h \models_{\Phi} x=\right.\text { null } \\
& \text { and }\left(s\left[\mathbf{z} \mapsto \mathbf{u}, z^{\prime} \mapsto v\right]\left\lceil z^{\prime}\right), h \models_{\Phi} z^{\prime} \neq\right. \text { null } \\
& \Leftrightarrow \text { [SL semantics] } \\
& \exists \mathbf{u} \in \operatorname{Val}^{\|\mathbf{z}\|} \text {. } \\
& \left(s[\mathbf{z} \mapsto \mathbf{u}] \Upsilon_{\mathbf{z}}\right), h \models_{\Phi} P \mathbf{z} \\
& \text { and } s(x)=\text { null and } s\left(z^{\prime}\right) \neq \text { null, }
\end{aligned}
$$

which coincides with

\section{A.15 Proof of Theorem 6}

We have to construct a heap automaton $\mathfrak{A}_{\mathrm{REACH}}$ over $\mathrm{SH}_{\mathrm{FV} \leq \alpha}$ that satisfies the compositionality property and accepts $\operatorname{REACH}(\alpha, R)$. In order to highlight the necessary proof obligations, the actual construction of $\mathfrak{A}_{\text {REACH }}$ and its correctness proof are splitted into several definitions and lemmas that are provided afterwards.

The construction of $\mathfrak{A}_{\text {REACH }}$ was already sketched in the paper. A formal construction is provided in Definition 19. It then remains to show the correctness of our construction of $\mathfrak{A}_{\text {REACH}}$ :

- Lemma 29 shows that $\mathfrak{A}_{\text {REACH }}$ indeed accepts $\operatorname{REACH}(\alpha, R)$, i.e., $L\left(\mathfrak{A}_{\mathrm{REACH}}\right)=$ $\operatorname{REACH}(\alpha, R)$.

- In order to prove that $\mathfrak{A}_{\text {REACH }}$ satisfies the compositionality property, we lift Lemma 19 to cover reachability as well. After that the compositionality property of $\mathfrak{A}_{\mathrm{REACH}}$ is verified analogously to the compositionality property of $\mathfrak{A}_{\text {TRACK }}$ (cf. Lemma 17).

The lifting of Lemma 19 is presented in Lemma 31. Similar to the proof of Lemma 18, the proof of Lemma 31 relies on an auxiliary property showing 
that

$$
x \rightsquigarrow \varphi\left[P_{1} / \tau\right] y \quad \text { iff } \quad x \rightsquigarrow \varphi\left[P_{1} / \operatorname{kernel}\left(P_{1} \mathbf{x}_{1},(B, \Lambda, S)\right)\right]
$$

holds for all $x, y \in \operatorname{Var}(\varphi)$ and symbolic heaps $\varphi$ containing a single predicate call $P_{1}$. This is formalized in Lemma 30.

Putting both together, we obtain a heap automaton $\mathfrak{A}_{\mathrm{REACH}}$ over $\mathrm{SH}_{\mathrm{FV}} \leq \alpha$ accepting $\operatorname{REACH}(\alpha, R)$.

Definition 19. Let $\mathbf{x}_{0}$ be a tuple of variables with $\left\|\mathbf{x}_{0}\right\|=\alpha \in \mathbb{N}_{>0}$. Then $\mathfrak{A}_{\mathrm{REACH}}=\left(Q, F V^{\leq \alpha}, \Delta, F\right)$ is given by

$$
\begin{aligned}
Q \triangleq & Q_{\mathfrak{A}_{\mathrm{REACH}}} \times 2^{\mathbf{x}_{0} \times \mathbf{x}_{0}}, \quad F \triangleq Q_{\mathfrak{A}_{\mathrm{TRACK}}} \times\{R\} \\
\Delta: & \mathbf{p} \stackrel{\varphi}{\rightarrow} \mathfrak{A}_{\mathrm{REACH}}\left(q_{0}, S_{0}\right), \mathbf{p}=\left(q_{1}, S_{1}\right) \ldots\left(q_{m}, S_{m}\right) \\
& \text { iff } q_{1} \ldots q_{m} \stackrel{\varphi}{\rightarrow} \mathfrak{A}_{\mathrm{TRACK}} q_{0} \\
& \text { and } \forall u, v \in \mathbf{x}_{0} \cdot(u, v) \in S_{0} \leftrightarrow u^{\varphi} \rightsquigarrow \operatorname{compress}(\varphi, \mathbf{p}) v^{\varphi},
\end{aligned}
$$

where

$$
\operatorname{compress}(\varphi, \mathbf{p}) \triangleq \varphi\left[P_{1} / \operatorname{kernel}\left(P_{1} \mathbf{x}_{1}, \mathbf{p}[1]\right), \ldots, P_{m} / \operatorname{kernel}\left(P_{m} \mathbf{x}_{m}, \mathbf{p}[m]\right)\right] .
$$

Here, $m=\left\|\Gamma^{\varphi}\right\|$ stands for the number of predicate calls occurring in $\varphi$ and $u^{\varphi}$ denotes the free variable of $\varphi$ corresponding to $u \in \mathbf{x}_{0} \cdot{ }^{15}$ Moreover, $q_{i}=$ $\left(A_{i}, \Pi_{i}\right) \in Q_{\mathfrak{A}_{\mathrm{TRACK}}}$ for each $0 \leq i \leq m$.

Lemma 29. $L\left(\mathfrak{A}_{\mathrm{REACH}}\right)=\operatorname{REACH}(\alpha, R)$.

Proof. Let $\tau \in \mathrm{RSH}_{\mathrm{FV} \leq \alpha}$. Then

$$
\begin{aligned}
& \tau \in L\left(\mathfrak{A}_{\mathrm{REACH}}\right) \\
\Leftrightarrow & {\left[\text { Definition of } L\left(\mathfrak{A}_{\mathrm{REACH}}\right)\right] } \\
& \exists q \in F_{\mathfrak{A}_{\mathrm{REACH}}} \cdot \varepsilon \stackrel{\tau}{\rightarrow}_{\mathfrak{A}_{\mathrm{REACH}}} q \\
\Leftrightarrow \quad & {\left[\text { Definition of } F_{\mathfrak{A}_{\mathrm{REACH}}}\right] } \\
& \exists p \in Q_{\mathfrak{A}_{\mathrm{TRACK}}} \cdot \varepsilon \stackrel{\tau}{\rightarrow}_{\mathfrak{A}_{\mathrm{REACH}}}(p, R) \\
\Leftrightarrow \quad & {\left[\text { Definition of } \Delta_{\mathfrak{A}_{\mathrm{REACH}}}\right] } \\
& \exists p \in Q_{\mathfrak{A}_{\mathrm{TRACK}}} \cdot \varepsilon \stackrel{\tau}{\rightarrow}_{\mathfrak{A}_{\mathrm{TRACK}}} p \\
\quad & \text { and } \forall u, v \in \mathbf{x}_{0} \cdot(u, v) \in R \leftrightarrow u^{\tau} \rightsquigarrow \operatorname{compress}(\tau, \varepsilon) \\
\Leftrightarrow \quad & {\left[\text { Definition } 19: v^{\tau} \operatorname{compress}(\tau, \varepsilon)=\tau\right] } \\
& \forall u, v \in \mathbf{x}_{0} \cdot(u, v) \in R \leftrightarrow u^{\tau} \rightsquigarrow v^{\tau} \\
\Leftrightarrow \quad & {[\text { Definition of } \operatorname{REACH}(\alpha, R)] } \\
& \tau \in \operatorname{REACH}(\alpha, R) .
\end{aligned}
$$

$\overline{{ }^{15} \text { formally if } u}=\mathbf{x}_{0}[i]$ then $u^{\varphi}=\mathbf{x}_{0}^{\varphi}[i]$ 
Lemma 30. Let $\varphi=\exists \mathbf{z} . \Sigma * P_{1} \mathbf{x}_{1}: \Pi \in \mathrm{SH}_{F V \leq \alpha}$. Moreover, let $\tau \in \mathrm{RSH}$ with $\left\|\mathbf{x}_{0}^{\tau}\right\|=\left\|\mathbf{x}_{1}^{\varphi}\right\|, B=\left\{y \in \mathbf{x}_{0} \mid y^{\tau} \in \operatorname{alloc}(\tau)\right\}, \Lambda=\left\{x \sim y \mid x^{\tau} \sim_{\tau} y^{\tau}\right\}$, and $S=\left\{(x, y) \in \mathbf{x}_{0} \times \mathbf{x}_{0} \mid x^{\tau} \rightsquigarrow_{\tau} y^{\tau}\right\}$. Then, for each $x, y \in \operatorname{Var}(\varphi)$, we have

$$
\begin{array}{rll}
x \sim_{\varphi\left[P_{1} / \tau\right]} y & \text { iff } & x \sim_{\varphi\left[P_{1} / \operatorname{kernel}\left(P_{1} \mathbf{x}_{1},(B, \Lambda, S)\right)\right]} y, \quad \text { and } \\
x \in \operatorname{alloc}\left(\varphi\left[P_{1} / \tau\right]\right) & \text { iff } & x \in \operatorname{alloc}\left(\varphi\left[P_{1} / \operatorname{kernel}\left(P_{1} \mathbf{x}_{1},(B, \Lambda, S)\right)\right]\right), \quad \text { and } \\
x \rightsquigarrow_{\varphi\left[P_{1} / \tau\right]} y & \text { iff } & x \rightsquigarrow_{\varphi\left[P_{1} / \operatorname{kernel}\left(P_{1} \mathbf{x}_{1},(B, \Lambda, S)\right)\right]} y
\end{array}
$$

where $\sigma_{P_{1} \mathbf{x}_{1},(B, \Lambda, S)}$ has been defined at the beginning of this section.

Proof. The proof of the first two equivalences is completely analogous to the proof of Lemma 18. Hence, it remains to prove that for each pair of variables $x, y \in \operatorname{Var}(\varphi)$, we have

$$
x \rightsquigarrow_{\varphi\left[P_{1} / \tau\right]} y \text { iff } x \rightsquigarrow_{\varphi}\left[P_{1} / \operatorname{kernel}\left(P_{1} \mathbf{x}_{1},(B, \Lambda, S)\right)\right] y .
$$

Since definite reachability always holds for unsatisfiable reduced symbolic heaps and, by the first equivalence, either both symbolic heaps are satisfiable or both are unsatisfiable, assume without loss of generality that $\varphi\left[P_{1} / \tau\right]$ is satisfiable. Both directions of the proposition from above are shown by induction on the number $n$ of definite points-to assertions $\mapsto_{\tau}$ needed to reach $y$ from $x$ in $x \rightsquigarrow \varphi\left[P_{1} / \tau\right] y$ and $x \rightsquigarrow_{\varphi\left[P_{1} / \operatorname{kernel}\left(P_{1} \mathbf{x}_{1},(B, \Lambda, S)\right)\right]} y$, respectively.

We first show that $x \rightsquigarrow_{\varphi\left[P_{1} / \tau\right]} y$ implies $x \rightsquigarrow_{\varphi\left[P_{1} / \operatorname{kernel}\left(P_{1} \mathbf{x}_{1},(B, \Lambda, S)\right)\right]} y$.

I.B. For the base case assume that $x \rightsquigarrow \varphi\left[P_{1} / \tau\right] y$ holds and $y$ is reachable by taking $n=1$ definite points-to assertions, i.e., $x \mapsto_{\varphi\left[P_{1} / \tau\right]} y$ holds. By Lemma 15 , this is equivalent to

$$
\begin{array}{r}
\exists z_{1}, z_{2} \cdot x=_{\varphi\left[P_{1} / \tau\right]} z_{1} \text { and } y={ }_{\varphi\left[P_{1} / \tau\right]} z_{2} \\
\text { and } z_{1} \mapsto\left(\left(-, z_{2},-\right)\right) \in \Sigma^{\varphi\left[P_{1} / \tau\right]} .
\end{array}
$$

Then, by definition of predicate replacement, two cases arise:

1. Case: $z_{1} \mapsto\left(\left(-, z_{2}, \_\right)\right) \in \Sigma^{\varphi}$. Then $z_{1}, z_{2} \in \operatorname{Var}(\varphi)$ and thus, by the already known first property of Lemma 30, we have $x={ }_{\varphi\left[P_{1} / \operatorname{kernel}\left(P_{1} \mathbf{x}_{1},(B, \Lambda, S)\right)\right]} z_{1}$ and $y={ }_{\varphi\left[P_{1} / \operatorname{kernel}\left(P_{1} \mathbf{x}_{1},(B, \Lambda, S)\right)\right]} z_{2}$. Since $\Sigma^{\varphi}$ is contained in the symbolic heap $\Sigma^{\varphi\left[P_{1} / \operatorname{kernel}\left(P_{1} \mathbf{x}_{1},(B, A, S)\right)\right]}$, this is means that

$$
x \mapsto_{\varphi\left[P_{1} / \operatorname{kernel}\left(P_{1} \mathbf{x}_{1},(B, \Lambda, S)\right)\right]} y .
$$

2. Case: $z_{1} \mapsto\left(\left(-, z_{2},-\right)\right) \in \Sigma^{\tau}$. Then $z_{1}, z_{2} \in \operatorname{Var}(\tau)$. By Lemma 28, there exist two variables $u, v \in \mathbf{x}_{1}^{\varphi}$ such that $x=_{\varphi\left[P_{1} / \tau\right]} u, u==_{\tau} z, y={ }_{\varphi\left[P_{1} / \tau\right]} v$, and $v={ }_{\tau} z^{\prime}$. Thus, $u \mapsto_{\tau} v$ holds. In particular, this means that $(u, v) \in S$ and thus $u \mapsto((-, v,-)) \in \Sigma^{\varphi\left[P_{1} / \operatorname{kernel}\left(P_{1} \mathbf{x}_{1},(B, A, S)\right)\right]}$. Now, by the already known first property of Lemma 30, we also have $x={ }_{\varphi\left[P_{1} / \operatorname{kernel}\left(P_{1} \mathbf{x}_{1},(B, \Lambda, S)\right)\right]} u$ and $y={ }_{\varphi\left[P_{1} / \operatorname{kernel}\left(P_{1} \mathbf{x}_{1},(B, \Lambda, S)\right)\right]} v$. Hence, $x \mapsto_{\varphi\left[P_{1} / \operatorname{kernel}\left(P_{1} \mathbf{x}_{1},(B, \Lambda, S)\right)\right]} y$. 
Thus, $x \rightsquigarrow_{\varphi\left[P_{1} / \operatorname{kernel}\left(P_{1} \mathbf{x}_{1},(B, \Lambda, S)\right)\right]} y$ holds in both cases.

I.H. Assume for an arbitrary, but fixed, natural number $n$ that $x \rightsquigarrow \varphi\left[P_{1} / \tau\right]$ $y$, where at most $n$ points-to assertions are used to reach $y$ from $x$, implies $x \rightsquigarrow \varphi\left[P_{1} / \operatorname{kernel}\left(P_{1} \mathbf{x}_{1},(B, \Lambda, S)\right)\right]$.

I.S. Assume $n+1$ definite points-to assertions are needed to reach $y$ from $x$ in $\varphi\left[P_{1} / \tau\right]$. By Lemma 15, there exists $z \in \operatorname{Var}\left(\varphi\left[P_{1} / \tau\right]\right)$ such that $x \mapsto_{\varphi\left[P_{1} / \tau\right]} z$ and $z \rightsquigarrow \varphi\left[P_{1} / \tau\right.$, where at most $n$ definite points-to assertions are needed to reach $y$ from $z$. Thus, by I.H. we know that $z \rightsquigarrow_{\varphi}\left[P_{1} / \operatorname{kernel}\left(P_{1} \mathbf{x}_{1},(B, \Lambda, S)\right)\right] y$. Furthermore, by the same argument as in the base case, we obtain $x \mapsto_{\varphi\left[P_{1} / \operatorname{kernel}\left(P_{1} \mathbf{x}_{1},(B, \Lambda, S)\right)\right]}$ $z$. Putting both together yields $x \rightsquigarrow_{\varphi\left[P_{1} / \operatorname{kernel}\left(P_{1} \mathbf{x}_{1},(B, \Lambda, S)\right)\right]} y$.

The proof of the converse direction is analogous. The only difference is that we obtain $x \rightsquigarrow \varphi\left[P_{1} / \tau\right]$ instead of $x \mapsto_{\varphi\left[P_{1} / \tau\right]} y$ in the case analysis of the base case, because $u \mapsto\left(\left(-, v_{-},\right)\right) \in \Sigma^{\text {kernel }\left(P_{1} \mathbf{x}_{1},(B, A, S)\right)}$ implies $u \rightsquigarrow_{\tau} v$ only.

Lemma 31. Let $\varphi \in \mathrm{SH}_{F V \leq \alpha}$ with $\left\|\Gamma^{\varphi}\right\|=m \geq 0$. For each $1 \leq i \leq m$, let $\tau_{i} \in \mathrm{RSH}_{F V \leq \alpha}$ with $\left\|\mathbf{x}_{0}^{\tau_{i}}\right\|=\left\|\mathbf{x}_{i}^{\varphi}\right\|, A_{i}=\left\{y \in \mathbf{x}_{0} \mid y^{\tau_{i}} \in \operatorname{alloc}\left(\tau_{i}\right)\right\}, \Pi_{i}=\{x \sim$ $\left.y \mid x^{\tau_{i}} \sim_{\tau_{i}} y^{\tau_{i}}\right\}$ and $S_{i}=\left\{(x, y) \in \mathbf{x}_{0} \times \mathbf{x}_{0} \mid x^{\tau_{i}} \rightsquigarrow \tau_{i} y^{\tau_{i}}\right\}$. Moreover, let

$$
\begin{aligned}
\tau & \triangleq \varphi\left[P_{1} / \tau_{1}, \ldots, P_{m} / \tau_{m}\right], \\
\mathbf{p} & \triangleq\left(A_{1}, \Pi_{1}, S_{1}\right) \ldots\left(A_{m}, \Pi_{m}, S_{m}\right), \text { and } \\
\operatorname{compress}(\varphi, \mathbf{p}) & \triangleq \varphi\left[P_{1} / \operatorname{kernel}\left(P_{1} \mathbf{x}_{1}, \mathbf{p}[1]\right), \ldots, P_{m} / \operatorname{kernel}\left(P_{m} \mathbf{x}_{m}, \mathbf{p}[m]\right)\right] .
\end{aligned}
$$

Then, for each $x, y \in \operatorname{Var}(\varphi)$, we have

$$
\begin{aligned}
& x \sim_{\tau} y \text { iff } x \sim_{\operatorname{compress}(\varphi, \mathbf{p})} y, \\
& x \in \operatorname{alloc}(\tau) \text { iff } x \in \operatorname{alloc}(\operatorname{compress}(\varphi, \mathbf{p})), \\
& x \rightsquigarrow_{\tau} y \text { iff } x \rightsquigarrow_{\operatorname{compress}(\varphi, \mathbf{p})} y .
\end{aligned}
$$

Proof. Analogous to the proof of Lemma 19 except for the use of Lemma 30 instead of Lemma 18.

\section{A.16 Proof of Theorem 7}

Upper bounds are obtained as in the proof of Theorem 5 . Thus, we only show that the reduction provided in the paper proving the lower bounds is correct. We first briefly recall how $\overline{\text { SL-RSAT }}$ is reduced to SL-REACH. Let $(\Phi, P)$ be an instance of $\overline{\mathrm{SL}-\mathrm{RSAT}}$. Then an instance of SL-REACH is given by $\left(\Phi, \varphi, \mathbf{x}_{0}[1], \mathbf{x}_{0}[2]\right)$, where

$$
\begin{aligned}
\varphi \triangleq \exists & \mathbf{z} \cdot \mathbf{x}_{0}[1] \mapsto(\text { null }) * P \mathbf{z}:\left\{\mathbf{x}_{0}[2] \neq \text { null }\right\} . \text { Now } \\
& \forall \tau \in \mathbb{U}_{\Phi}(\varphi) \cdot \mathbf{x}_{0}[1] \rightsquigarrow_{\tau} \mathbf{x}_{0}[2] \\
\Leftrightarrow & {[\text { Construction of } \varphi,(\tau \text { contains one points-to assertion })] } \\
& \forall \tau \in \mathbb{U}_{\Phi}(\varphi) \cdot \mathbf{x}_{0}[1] \mapsto_{\tau} \mathbf{x}_{0}[2]
\end{aligned}
$$




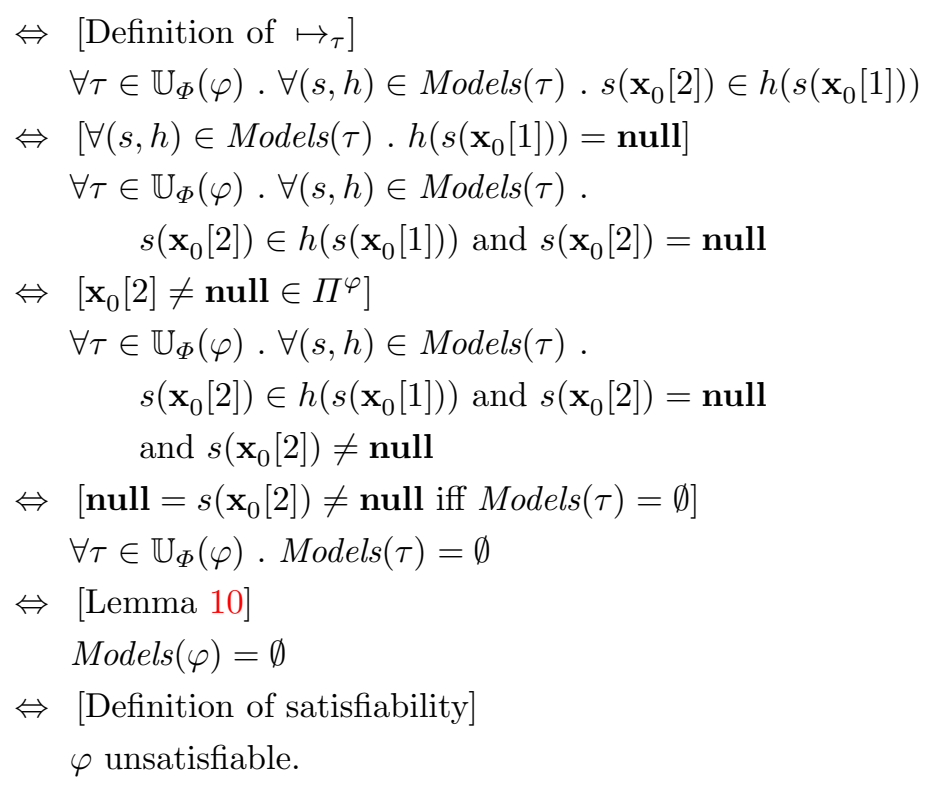

Since $\varphi$ is satisfiable if and only if $P$ is satisfiable by an analogous argument as in the proof of Lemma 4, it follows that $\mathbf{x}_{0}[1] \rightsquigarrow_{\varphi} \mathbf{x}_{0}[2]$ holds for each $\tau \in \mathbb{U}_{\Phi}(\varphi)$ if and only if $P$ is unsatisfiable.

\section{A.17 Proof of Lemma 6}

We have to construct a heap automaton $\mathfrak{A}_{\text {GFREE }}$ over $\mathrm{SH}_{\mathrm{FV} \leq \alpha}$ that satisfies the compositionality property and accepts $\operatorname{GFREE}(\alpha)$. In order to highlight the necessary proof obligations, the actual construction of $\mathfrak{A}_{\text {GFREE }}$ and its correctness proof are splitted into several definitions and lemmas that are provided afterwards.

The construction of $\mathfrak{A}_{\text {GFREE }}$ is similar to the construction of $\mathfrak{A}_{\mathrm{EST}}$ as presented in Theorem 4. The main difference is that the reachability automaton $\mathfrak{A}_{\text {REACH}}$, formally introduced in Definition 19, is used instead of the tracking automaton $\mathfrak{A}_{\text {TRACK }}$ and that the predicate check is adapted. A fully formal construction of $\mathfrak{A}_{\text {GFREE }}$ is found in Definition 20. It then remains to show the correctness of our construction of $\mathfrak{A}_{\mathrm{GFREE}}$ :

- Lemma 32 establishes that $\mathfrak{A}_{\text {GFREE }}$ indeed accepts $\operatorname{GFREE}(\alpha)$, i.e., $L\left(\mathfrak{A}_{\text {GFREE }}\right)=$ $\operatorname{GFREE}(\alpha)$.

- To prove the compositionality property, we show that $\mathfrak{A}_{\text {GFREE }}$ is an instance of a more general construction scheme whose compositionality property is shown in Lemma 25. In order to apply Lemma 25, we have to show that

$$
\begin{aligned}
& \quad \operatorname{check}\left(\varphi\left[P_{1}^{\varphi} / \tau_{1}, \ldots, P_{m}^{\varphi} / \tau_{m}\right], \varepsilon\right)=1 \\
& \text { iff } \operatorname{check}\left(\varphi, p_{1} \ldots p_{m}\right)=1 \\
& \quad \text { and } \operatorname{check}\left(\tau_{1}, \varepsilon\right)=\ldots=\operatorname{check}\left(\tau_{m}, \varepsilon\right)=1 .
\end{aligned}
$$


This is verified in Lemma 33. Then, by Lemma 25, we know that $\mathfrak{A}_{\mathrm{GFREE}}=$ $\mathfrak{A}_{\mathrm{SCHEME}}\left(\mathfrak{A}_{\mathrm{REACH}}\right.$, check,$\left.F\right)$ satisfies the compositionality property.

Putting both together, we obtain a heap automaton $\mathfrak{A}_{\text {GFREE }}$ over $\mathrm{SH}_{\mathrm{FV} \leq \alpha}$ that satisfies the compositionality property and accepts $\operatorname{GFREE}(\alpha)$.

Definition 20. $\mathfrak{A}_{\mathrm{GFREE}}=\left(Q, \mathrm{SH}_{F V \leq \alpha}, \Delta, F\right)$ is given by

$$
\begin{aligned}
& Q \triangleq Q_{\mathfrak{A}_{\mathrm{REACH}}} \times\{0,1\} \quad F \triangleq Q_{\mathfrak{A}_{\mathrm{REACH}}} \times\{1\} \\
& \Delta:\left(p_{1}, q_{1}\right) \ldots\left(p_{m}, q_{m}\right) \stackrel{\varphi}{\rightarrow} \mathfrak{A}_{\mathrm{GFREE}}\left(p_{0}, q_{0}\right) \\
& \text { iff } p_{1} \ldots p_{m} \stackrel{\varphi}{\rightarrow}_{\mathfrak{A}_{\mathrm{REACH}}} p_{0} \\
& \quad \text { and } q_{0}=\min \left\{q_{1}, \ldots, q_{m}, \operatorname{check}\left(\varphi, p_{1} \ldots p_{m}\right)\right\},
\end{aligned}
$$

where $\mathfrak{A}_{\mathrm{REACH}}$ is as in Definition 19. Furthermore, the predicate check: $\mathrm{SH}_{F V \leq \alpha} \times$ $Q_{\mathfrak{A}_{\mathrm{REACH}}}^{*} \rightarrow\{0,1\}$ verifies that each variable of a symbolic heap $\varphi$ is established in compress $(\varphi, \mathbf{p})$, where compress $(\varphi, \mathbf{p})$ is the same as in the construction of $\mathfrak{A}_{\mathrm{REACH}}$. Hence,

$$
\operatorname{check}(\varphi, \mathbf{p}) \triangleq\left\{\begin{array}{c}
1 \quad, \quad \text { if } \forall y \in \operatorname{Var}(\varphi) \cdot \exists x \in \mathbf{x}_{0}^{\varphi} . \\
\quad x=\operatorname{compress}(\varphi, \mathbf{p}) y \text { or } x \rightsquigarrow \operatorname{compress}(\varphi, \mathbf{p}) y \\
0 \quad, \text { otherwise }
\end{array}\right.
$$

Lemma 32. $L\left(\mathfrak{A}_{\text {GFREE }}\right)=\operatorname{GFREE}(\alpha)$.

Proof. Let $\tau \in \mathrm{RSH}_{\mathrm{FV} \leq \alpha}$. Then:

$\tau \in L\left(\mathfrak{A}_{\text {GFREE }}\right)$

$\Leftrightarrow \quad\left[\right.$ Definition of $\left.L\left(\mathfrak{A}_{\mathrm{GFREE}}\right)\right]$

$\exists q \in F_{\mathfrak{A}_{\mathrm{GFREE}}} \cdot \varepsilon \stackrel{\tau}{\rightarrow}_{\mathfrak{A}_{\mathrm{GFRE}}} q$

$\Leftrightarrow \quad$ [Definition of $\left.F_{\mathfrak{A}_{\text {GFRE }}}, q=(p, 1)\right]$

$\exists p \in Q_{\mathfrak{A}_{\mathrm{REACH}}} \cdot \varepsilon \stackrel{\tau}{\rightarrow}_{\mathfrak{A}_{\mathrm{GFREE}}}(p, 1)$

$\Leftrightarrow$ [Definition of $\left.\Delta_{\mathfrak{A}_{\mathrm{GFRE}}}\right]$

$\exists p \in Q_{\mathfrak{A}_{\mathrm{REACH}}} \cdot \varepsilon \stackrel{\tau}{\rightarrow} \mathfrak{A}_{\mathrm{REACH}} p$ and $\operatorname{check}(\operatorname{compress}(\tau, \varepsilon))=1$

$\Leftrightarrow \quad[$ Definition of check(compress $(\tau, \varepsilon))]$

$\exists p \in Q_{\mathfrak{A}_{\mathrm{REACH}}} \cdot \varepsilon \stackrel{\tau}{\rightarrow} \mathfrak{A}_{\mathrm{REACH}} p$

and $\forall y \in \operatorname{Var}(\tau) . \exists x \in \mathbf{x}_{0}^{\tau} \cdot x=\operatorname{compress}(\tau, \varepsilon)_{y}$

or $x \rightsquigarrow \operatorname{compress}(\tau, \varepsilon) y$

$\Leftrightarrow \quad\left[\left\|\Gamma^{\tau}\right\|=0\right.$ implies $\left.\tau=\operatorname{compress}(\tau, \varepsilon)\right]$

$\forall y \in \operatorname{Var}(\tau) . \exists x \in \mathbf{x}_{0}^{\tau} \cdot x={ }_{\tau} y$ or $x \rightsquigarrow_{\tau} y$

$\Leftrightarrow \quad[$ Definition of $\operatorname{GFREE}(\alpha)]$

$\tau \in \operatorname{GFREE}(\alpha)$. 
Lemma 33. Let $\varphi \in \mathrm{SH}_{F V \leq \alpha}$ with $\left\|\Gamma^{\varphi}\right\|=m$ and $\tau_{1}, \ldots, \tau_{m} \in \mathrm{RSH}_{F V \leq \alpha}$. Then

$$
\begin{aligned}
& \operatorname{check}\left(\varphi\left[P_{1}^{\varphi} / \tau_{1}, \ldots, P_{m}^{\varphi} / \tau_{m}\right], \varepsilon\right)=1 \\
& \text { iff } \operatorname{check}(\varphi, \mathbf{p}) \\
& \quad \text { and } \operatorname{check}\left(\tau_{1}, \varepsilon\right)=\ldots=\operatorname{check}\left(\tau_{m}, \varepsilon\right)=1 .
\end{aligned}
$$

The following technical observation, similar to Lemma 28 , is essential. Intuitively, it states that a variable in one part of an unfolding is definitely reachable by a variable in a different part of an unfolding only through a parameter (including null) of one or more suitable predicate calls. This is illustrated in Figure 2.

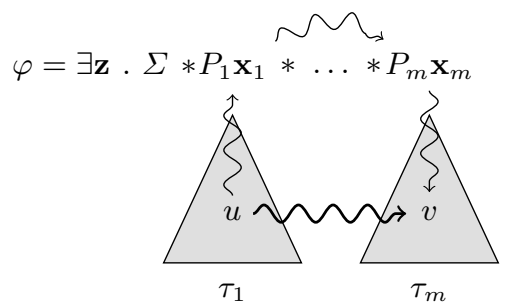

Fig. 2. Propagation of reachability through parameters in a symbolic heap $\tau=$ $\varphi\left[P_{1} / \tau_{1}, \ldots, P_{m} / \tau_{m}\right]$. Here, $u \rightsquigarrow_{\tau} v$ holds (thick arrow). Since $u \in \operatorname{Var}\left(\tau_{1}\right)$ and $v \in \operatorname{Var}\left(\tau_{m}\right)$, there exist parameters $x, y$ of $P_{1}$ and $P_{m}$ that $u \rightsquigarrow_{\tau_{1}} x, x \rightsquigarrow_{\tau} y$, and $y \rightsquigarrow \tau_{m} v$ (thin arrows).

Lemma 34. Let $\varphi \in \mathrm{SH}_{F V \leq \alpha}$ with $\left\|\Gamma^{\varphi}\right\|=m, \tau_{1}, \ldots, \tau_{m} \in \mathrm{RSH}_{F V \leq \alpha}$ and $\tau=$ $\varphi\left[P_{1}^{\varphi} / \tau_{1}, \ldots, P_{m}^{\varphi} / \tau_{m}\right]$. Moreover, for some $1 \leq i \leq m$, let $x \in \operatorname{Var}\left(\tau_{i}\left[\mathbf{x}_{0}^{\tau_{i}} / \mathbf{x}_{i}^{\varphi}\right]\right)$ and $y \in \operatorname{Var}(\tau) \backslash \operatorname{Var}\left(\tau_{i}\left[\mathbf{x}_{0}^{\tau_{i}} / \mathbf{x}_{i}^{\varphi}\right]\right)$. Then $x \rightsquigarrow_{\tau} y$ holds if and only if there exists $z \in \mathbf{x}_{i}^{\varphi}$ such that

$$
\begin{aligned}
& -x={ }_{\tau_{i}\left[\mathbf{x}_{0}^{\tau_{i}} / \mathbf{x}_{i}^{\varphi}\right]} z \text { and and } z \rightsquigarrow_{\tau} y \text {, or } \\
& -x{\tau_{i}\left[\mathbf{x}_{0}^{\tau_{i}} / \mathbf{x}_{i}^{\varphi}\right]} z \text { and } z={ }_{\tau} y \text {, or } \\
& -x \rightsquigarrow_{\tau_{i}}\left[\mathbf{x}_{0}^{\tau_{i}} / \mathbf{x}_{i}^{\varphi}\right] \\
& - \text { and } z \rightsquigarrow_{\tau} y .
\end{aligned}
$$

The same holds for the converse direction, i.e., if $y \in \operatorname{Var}\left(\tau_{i}\left[\mathbf{x}_{0}^{\tau_{i}} / \mathbf{x}_{i}^{\varphi}\right]\right)$ and $x \in$ $\operatorname{Var}(\tau) \backslash \operatorname{Var}\left(\tau_{i}\left[\mathbf{x}_{0}^{\tau_{i}} / \mathbf{x}_{i}^{\varphi}\right]\right)$.

Proof (sketch). The implication from right to left is straightforward. The implication from left to right is shown by a lengthy complete induction on the number of definitive points-to assertions to reach $y$ from $x$.

Note that this observation relies on the fact that the reachability relation $\rightsquigarrow \varphi$ is defined with respect to all models of $\varphi$, not just a single one. Otherwise, the observation is wrong for symbolic heaps that are not established. 
Proof (of Lemma 33). Let $\tau=\operatorname{check}\left(\varphi\left[P_{1}^{\varphi} / \tau_{1}, \ldots, P_{m}^{\varphi} / \tau_{m}\right]\right)$. Then:

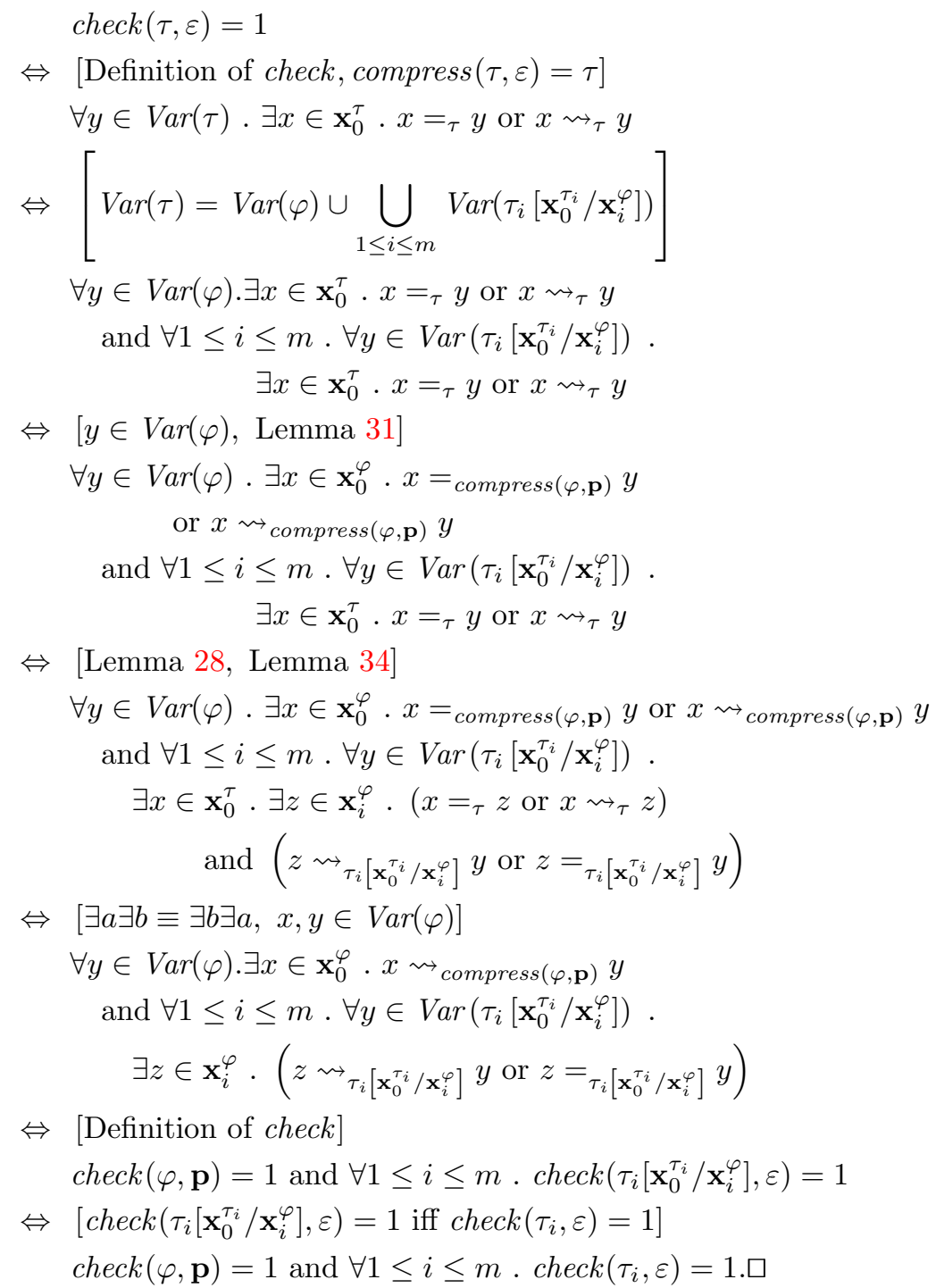

\section{A.18 Proof of Theorem 8}

Since upper bounds are obtained analogously to Theorem 5, we only show that the reduction provided in the paper proving the lower bounds is correct. We first briefly recall how $\overline{\mathrm{SL}-\mathrm{RSAT}}$ is reduced to SL-GF. Let $(\Phi, P)$ be an instance of $\overline{\text { SL-RSAT. }}$. Then a corresponding instance of SL-GF is given by $(\Phi, \varphi)$, where 


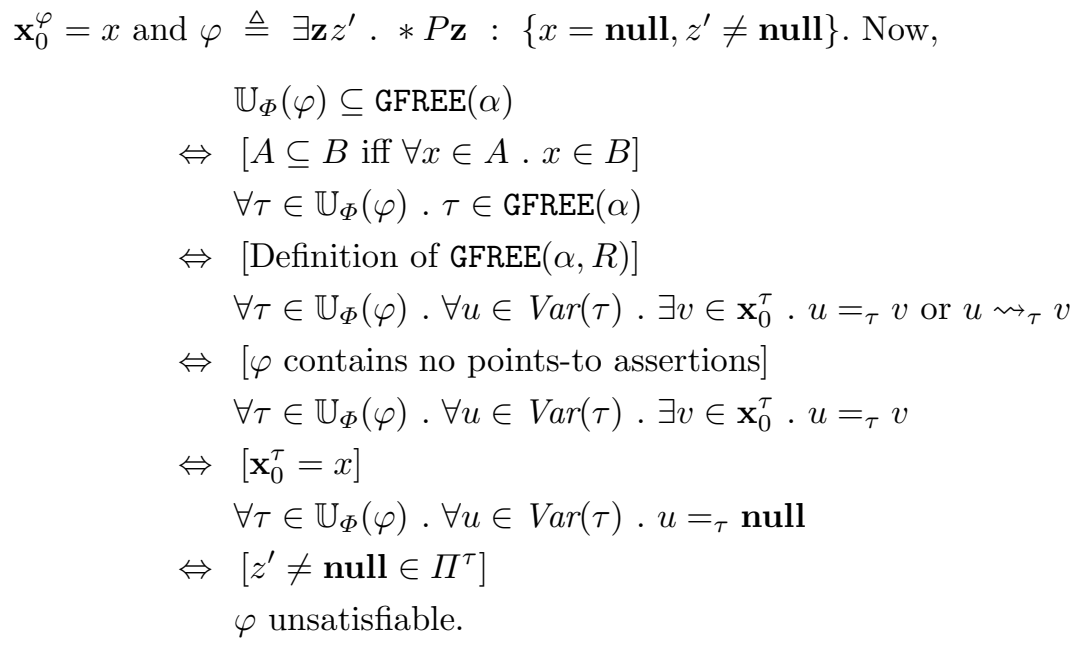

Since $\varphi$ is satisfiable if and only if $P$ is satisfiable by an analogous argument as in the proof of Lemma 4 , it follows that $\mathbb{U}_{\Phi}(\varphi) \subseteq \operatorname{GFREE}(\alpha)$ holds if and only if $P$ is unsatisfiable.

\section{A.19 Proof of Lemma 7}

We have to construct a heap automaton $\mathfrak{A}_{\mathrm{ACYCLIC}}$ over $\mathrm{SH}_{\mathrm{FV} \leq \alpha}$ that satisfies the compositionality property and accepts $\operatorname{ACYCLIC}(\alpha)$. In order to highlight the necessary proof obligations, the actual construction of $\mathfrak{A}_{\text {ACYCLIC }}$ and its correctness proof are splitted into several definitions and lemmas that are provided afterwards.

The construction of $\mathfrak{A}_{\text {ACYCLIC }}$ is similar to the construction of $\mathfrak{A}_{\text {EST }}$ as presented in Theorem 4 . The main difference is that the reachability automaton $\mathfrak{A}_{\text {REACH }}$, formally introduced in Definition 19, is used instead of the tracking automaton $\mathfrak{A}_{\text {TRACK }}$ and that the predicate check is adapted. A fully formal construction of $\mathfrak{A}_{\text {ACYCLIC }}$ is found in Definition 21. It then remains to show the correctness of our construction of $\mathfrak{A}_{\mathrm{ACYCLIC}}$ :

- Lemma 35 establishes that $\mathfrak{A}_{\text {ACYCLIC }}$ indeed accepts $\operatorname{ACYCLIC}(\alpha)$, i.e., we have $L\left(\mathfrak{A}_{\mathrm{ACYCLIC}}\right)=\operatorname{ACYCLIC}(\alpha)$.

- To prove the compositionality property, we show that $\mathfrak{A}_{\text {ACYCLIC }}$ is an instance of a more general construction scheme whose compositionality property is shown in Lemma 25. In order to apply Lemma 25, we have to show that

$$
\begin{aligned}
& \operatorname{check}\left(\varphi\left[P_{1}^{\varphi} / \tau_{1}, \ldots, P_{m}^{\varphi} / \tau_{m}\right], \varepsilon\right)=1 \\
& \text { iff } \operatorname{check}\left(\varphi, p_{1} \ldots p_{m}\right)=1 \\
& \quad \text { and } \operatorname{check}\left(\tau_{1}, \varepsilon\right)=\ldots=\operatorname{check}\left(\tau_{m}, \varepsilon\right)=1 .
\end{aligned}
$$

This is verified in Lemma 36 . Then, by Lemma 25 , we know that $\mathfrak{A}_{\text {ACYCLIC }}=$ $\mathfrak{A}_{\mathrm{SCHEME}}\left(\mathfrak{A}_{\mathrm{REACH}}\right.$, check,$\left.F\right)$ satisfies the compositionality property. 
Putting both together, we obtain a heap automaton $\mathfrak{A}_{\text {ACYCLIC }}$ over $\mathrm{SH}_{\mathrm{FV} \leq \alpha}$ that satisfies the compositionality property and accepts $\operatorname{ACYCLIC}(\alpha)$.

Definition 21. $\mathfrak{A}_{\mathrm{ACYCLIC}}=\left(Q, \mathrm{SH}_{F V \leq \alpha}, \Delta, F\right)$ is given by

$$
\begin{aligned}
& Q \triangleq Q_{\mathfrak{A}_{\mathrm{REACH}}} \times\{0,1\} \\
& F \triangleq Q_{\mathfrak{A}_{\mathrm{REACH}}} \times\{1\} \\
& \cup\left\{(A, \Pi, S) \in Q_{\mathfrak{A}_{\mathrm{REACH}}} \mid \text { null } \neq \text { null } \in \Pi\right\} \times\{0\} \\
& \Delta \quad\left(p_{1}, q_{1}\right) \ldots\left(p_{m}, q_{m}\right) \stackrel{\varphi}{\rightarrow}_{\mathfrak{A}_{\mathrm{ACYCLIC}}}\left(p_{0}, q_{0}\right) \\
& \text { iff } p_{1} \ldots p_{m} \stackrel{\varphi}{\rightarrow} \mathfrak{A}_{\mathrm{REACH}} p_{0} \\
& \text { and } q_{0}=\min \left\{q_{1}, \ldots, q_{m}, \operatorname{check}\left(\varphi, p_{1} \ldots p_{m}\right)\right\},
\end{aligned}
$$

where $\mathfrak{A}_{\mathrm{REACH}}$ is is as in Definition 19. Furthermore, the predicate check: $\mathrm{SH}_{F V \leq \alpha} \times Q_{\mathfrak{A}_{\mathrm{REACH}}}^{*} \rightarrow\{0,1\}$ verifies that each variable of a symbolic heap $\varphi$ is not reachable from itself in compress $(\varphi, \mathbf{p})$, where $\operatorname{compress}(\varphi, \mathbf{p})$ is the same as in the construction of $\mathfrak{A}_{\mathrm{REACH}}$. Hence,

$$
\operatorname{check}(\varphi, \mathbf{p}) \triangleq \begin{cases}1 & , \text { if } \forall y \in \operatorname{Var}(\varphi) . \operatorname{not} x \rightsquigarrow \operatorname{compress}(\varphi, \mathbf{p}) x \\ 0 & , \text { otherwise. }\end{cases}
$$

Lemma 35. $L\left(\mathfrak{A}_{\mathrm{ACYCLIC}}\right)=\operatorname{ACYCLIC}(\alpha)$.

Proof. Let $\tau \in \mathrm{RSH}_{\mathrm{FV} \leq \alpha}$. Then:

$$
\begin{aligned}
& \tau \in L\left(\mathfrak{A}_{\text {ACYCLIC }}\right) \\
& \left.\Leftrightarrow \quad \text { [Definition of } L\left(\mathfrak{A}_{\text {ACYCLIC }}\right)\right] \\
& \exists q \in F_{\mathfrak{A}_{\mathrm{ACYCLIC}}} \cdot \varepsilon \stackrel{\tau}{\rightarrow}_{\mathfrak{A}_{\mathrm{ACYCLIC}}} q \\
& \left.\Leftrightarrow \quad \text { [Definition of } F_{\mathfrak{A}_{\text {ACYCLIC }}},\right] \\
& \exists p \in Q_{\mathfrak{A}_{\text {REACH }}} . \varepsilon \stackrel{\tau}{\rightarrow}_{\mathfrak{A}_{\text {ACYCLIC }}}(p, 1) \\
& \text { or } \exists p=(A, \Pi, S) \in Q_{\mathfrak{A}_{\text {REACH }}} . \exists r \in\{0,1\} \text {. } \\
& \text { null } \neq \text { null } \in \Pi \text { and } \varepsilon \stackrel{\tau}{\rightarrow} \mathfrak{A}_{\text {ACYCLIC }}(p, r) \\
& \left.\Leftrightarrow \quad \text { [Definition of } \Delta_{\mathfrak{A}_{\text {ACYCLIC }}}\right] \\
& \exists p \in Q_{\mathfrak{A}_{\mathrm{REACH}}} \cdot \varepsilon \stackrel{\tau}{\rightarrow} \mathfrak{A}_{\mathrm{REACH}} p \text { and } \operatorname{check}(\tau, \varepsilon)=1 \\
& \text { or } \exists p=(A, \Pi, S) \in Q_{\mathfrak{A}_{\mathrm{REACH}}} . \exists r \in\{0,1\} \text {. } \\
& \text { null } \neq \text { null } \in \Pi \text { and } \varepsilon \stackrel{\tau}{\rightarrow} \mathfrak{A}_{\text {REACH }} p \text { and } \operatorname{check}(\tau, \varepsilon)=r \\
& \Leftrightarrow[\operatorname{check}(\tau, \varepsilon) \in\{0,1\}] \\
& \exists p \in Q_{\mathfrak{A}_{\mathrm{REACH}}} \cdot \varepsilon \stackrel{\tau}{\rightarrow}_{\mathfrak{A}_{\mathrm{REACH}}} p \text { and } \operatorname{check}(\tau, \varepsilon)=1 \\
& \text { or } \exists p=(A, \Pi, S) \in Q_{\mathfrak{A}_{\mathrm{REACH}}} \text {. null } \neq \text { null } \in \Pi \text { and } \varepsilon \stackrel{\tau}{\rightarrow}_{\mathfrak{A}_{\mathrm{REACH}}} p \\
& \Leftrightarrow \quad \text { [Definition of check] } \\
& \exists p \in Q_{\mathfrak{A}_{\mathrm{REACH}}} \cdot \varepsilon \stackrel{\tau}{\rightarrow}_{\mathfrak{A}_{\mathrm{REACH}}} p
\end{aligned}
$$




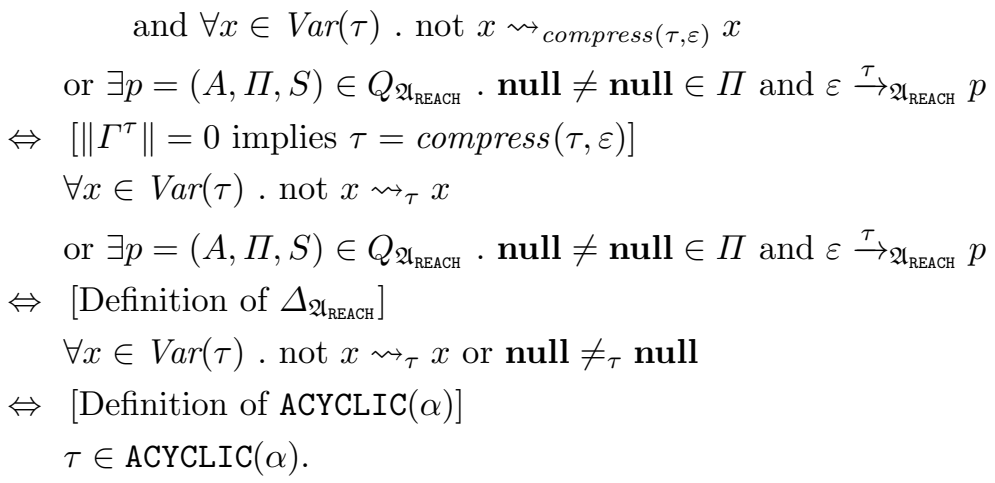

Lemma 36. Let $\varphi \in \mathrm{SH}_{F V \leq \alpha}$ with $\left\|\Gamma^{\varphi}\right\|=m$ and $\tau_{1}, \ldots, \tau_{m} \in \mathrm{RSH}_{F V \leq \alpha}$. Then

$$
\begin{aligned}
& \operatorname{check}\left(\varphi\left[P_{1}^{\varphi} / \tau_{1}, \ldots, P_{m}^{\varphi} / \tau_{m}\right], \varepsilon\right)=1 \\
& \text { iff } \operatorname{check}(\varphi, \mathbf{p}) \\
& \quad \text { and } \operatorname{check}\left(\tau_{1}, \varepsilon\right)=\ldots=\operatorname{check}\left(\tau_{m}, \varepsilon\right)=1 .
\end{aligned}
$$

Proof. Let $\tau=\operatorname{check}\left(\varphi\left[P_{1}^{\varphi} / \tau_{1}, \ldots, P_{m}^{\varphi} / \tau_{m}\right]\right)$. Then

$$
\begin{aligned}
& \operatorname{check}(\tau, \varepsilon)=1 \\
\Leftrightarrow \quad & {[\operatorname{Definition} \text { of } \operatorname{check}, \tau=\operatorname{compress}(\tau, \varepsilon)] } \\
& \forall x \in \operatorname{Var}(\tau) \cdot \operatorname{not} x \rightsquigarrow_{\tau} x \\
\Leftrightarrow & {\left[\operatorname{Var}(\tau)=\operatorname{Var}(\varphi) \cup \bigcup_{1 \leq i \leq m} \operatorname{Var}\left(\tau_{i}\left[\mathbf{x}_{0}^{\tau_{i}} / \mathbf{x}_{i}^{\varphi}\right]\right)\right] } \\
& \forall x \in \operatorname{Var}(\varphi) \cdot \operatorname{not} x \rightsquigarrow_{\tau} x \\
& \text { and } \forall 1 \leq i \leq m . \forall x \in \operatorname{Var}\left(\tau_{i}\left[\mathbf{x}_{0}^{\tau_{i}} / \mathbf{x}_{i}^{\varphi}\right]\right) \cdot \operatorname{not} x \rightsquigarrow_{\tau} x \\
\Leftrightarrow & {[\operatorname{Lemma} 30] } \\
& \forall x \in \operatorname{Var}(\varphi) \cdot \operatorname{not} x \rightsquigarrow_{\operatorname{compress}(\varphi, \mathbf{p})} x \\
& \text { and } \forall 1 \leq i \leq m . \forall x \in \operatorname{Var}\left(\tau_{i}\left[\mathbf{x}_{0}^{\tau_{i}} / \mathbf{x}_{i}^{\varphi}\right]\right) \cdot \operatorname{not} x \rightsquigarrow_{\tau} x .
\end{aligned}
$$

Assume towards a contradiction that $\operatorname{check}\left(\tau_{i}, \varepsilon\right)=0$ for some $1 \leq i \leq m$. By definition and compress $\left(\tau_{i}, \varepsilon\right)=\tau_{i}$, this means that $x \rightsquigarrow \tau_{i} x$. However, since $\tau_{i}$ is contained in $\tau$, this means that $x \rightsquigarrow \tau x$ and thus $\operatorname{check}(\tau, \varepsilon)=0$.

Conversely, assume $\operatorname{check}(\tau, \varepsilon)=0$, but $\operatorname{check}(\varphi, \mathbf{p})=1$ and $\operatorname{check}\left(\tau_{i}, \varepsilon\right)=1$ for each $1 \leq i \leq m$. Thus,

$$
\begin{aligned}
& \forall x \in \operatorname{Var}(\varphi) . \operatorname{not} x \rightsquigarrow \operatorname{compress}(\varphi, \mathbf{p}) \\
& \text { and } \forall 1 \leq i \leq m . \forall x \in \operatorname{Var}\left(\tau_{i}\left[\mathbf{x}_{0}^{\tau_{i}} / \mathbf{x}_{i}^{\varphi}\right]\right) . \operatorname{not} x \rightsquigarrow \tau_{i} x
\end{aligned}
$$

Then, there exists $x \in \operatorname{Var}(\tau)$ such that $x \rightsquigarrow_{\tau} x$. We proceed by case distinction: 
1. Case: $x \in \operatorname{Var}(\varphi)$. Then we immediately obtain a contradiction due to ( $)$ ).

2. Case: $x \rightsquigarrow \tau_{i}\left[\mathbf{x}_{0}^{\tau_{i}} / \mathbf{x}_{i}^{\varphi}\right]$ holds, we immediately obtain a contradiction due to

3. Case: For some $1 \leq i \leq m, x \in \operatorname{Var}\left(\tau_{i}\left[\mathbf{x}_{0}^{\tau_{i}} / \mathbf{x}_{i}^{\varphi}\right]\right)$ and $x \rightsquigarrow \tau_{i}\left[\mathbf{x}_{0}^{\tau_{i}} / \mathbf{x}_{i}^{\varphi}\right]$ not hold. Then there exists $y \in \operatorname{Var}(\tau) \backslash \operatorname{Var}\left(\tau_{i}\left[\mathbf{x}_{0}^{\tau_{i}} / \mathbf{x}_{i}^{\varphi}\right]\right)$ such that one of the following two cases holds:

(a) $x={ }_{\tau} y$ and $y \rightsquigarrow_{\tau} y$. Then, by Lemma 28, there exists $z \in \mathbf{x}_{i}^{\varphi}$ such that $x={ }_{\tau_{i}\left[\mathbf{x}_{0}^{\tau_{i}} / \mathbf{x}_{i}^{\varphi}\right]} z$ and $z={ }_{\tau} y$. However, by construction of $\operatorname{compress}(\varphi, \mathbf{p})$, this means that $z \rightsquigarrow \operatorname{compress}(\varphi, \mathbf{p}) z$ holds, which contradicts ( $\mathbf{p})$.

(b) $x \rightsquigarrow_{\tau} y$ and $y \rightsquigarrow_{\tau} x$. Then, by Lemma 34, there exist $u, v \in \mathbf{x}_{i}^{\varphi}$ such that $u \rightsquigarrow_{\tau} v$ and $v \rightsquigarrow_{\tau} u$. However, by construction of $\operatorname{compress}(\varphi, \mathbf{p})$, this means that $u \rightsquigarrow \operatorname{compress}(\varphi, \mathbf{p}) u$ holds. Thus $\operatorname{check}(\varphi, \mathbf{p})=0$, which contradicts $(\boldsymbol{\beta})$.

Since each case leads to a contradiction, we conclude $\operatorname{check}(\tau, \mathbf{p})=1$.

\section{A.20 Proof of Theorem 9}

Upper bounds are obtained analogously to Theorem 5. Thus, we only show that the reduction provided in the paper proving the lower bounds is correct. We first briefly recall how $\overline{\mathrm{SL}-\mathrm{RSAT}}$ is reduced to SL-AC. Let $(\Phi, P)$ be an instance of $\overline{\mathrm{SL}-\mathrm{RSAT}}$. Then an instance of SL-AC is given by $(\Phi, \varphi)$, where $\mathbf{x}_{0}^{\varphi}=x$ and $\varphi=\exists \mathbf{z} . x \mapsto(x) * P \mathbf{z}$. Then

$$
\begin{aligned}
& \mathbb{U}_{\Phi}(\varphi) \subseteq \operatorname{ACYCLIC}(\alpha) \\
\Leftrightarrow & {[A \subseteq B \text { iff } \forall x \in A . x \in B] } \\
& \forall \tau \in \mathbb{U}_{\Phi}(\varphi) \cdot \tau \in \operatorname{ACYCLIC}(\alpha) \\
\Leftrightarrow & {[\text { Definition of ACYCLIC }(\alpha)] } \\
& \forall \tau \in \mathbb{U}_{\Phi}(\varphi) \cdot \text { null } \neq_{\tau} \text { null or } \forall y \in \operatorname{Var}(\tau) \text { not } y \rightsquigarrow_{\tau} y \\
\Leftrightarrow & {[\varphi \text { contains exactly one points-to assertion }] } \\
& \forall \tau \in \mathbb{U}_{\Phi}(\varphi) \cdot \text { null } \neq_{\tau} \text { null or not } x \rightsquigarrow_{\tau} x \\
\Leftrightarrow & {\left[x \rightsquigarrow_{\tau} x \text { always hold by construction of } \varphi\right] } \\
& \forall \tau \in \mathbb{U}_{\Phi}(\varphi) \cdot \text { null } \neq_{\tau} \text { null } \\
\Leftrightarrow & {\left[\text { null } \neq_{\tau} \text { null iff } \operatorname{Models}(\tau)=\emptyset\right] } \\
& \forall \tau \in \mathbb{U}_{\Phi}(\varphi) . \text { Models }(\tau)=\emptyset \\
\Leftrightarrow & {[\text { Definition } 7] } \\
& \varphi \text { unsatisfiable. }
\end{aligned}
$$

Now, by an analogous argument as in the proof of Theorem 5 , we obtain that $\varphi$ is satisfiable iff $P$ is satisfiable. Then $\mathbb{U}_{\Phi}(\varphi) \subseteq \operatorname{ACYCLIC}(\alpha)$ holds iff $P$ is

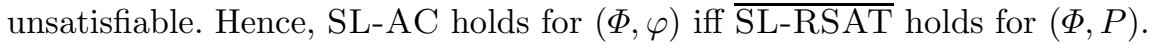




\section{A.21 Proof of Lemma 8}

The crux of the proof relies on the fact that each unfolding of $P_{1} \mathbf{x}$ has exactly one canonical model up to isomorphism, i.e.,

$$
\forall \sigma \in \mathbb{U}_{\Phi}\left(P_{1} \mathbf{x}\right) .\|\operatorname{Models}(\sigma)\|=1 .
$$

This property is a direct consequence of two properties: By Definition 11, $\sigma$ has at most one canonical model up to isomorphism, because $\Phi$ is determined. Furthermore, each symbolic heap $\sigma \in \mathbb{U}_{\Phi}\left(P_{1} \mathbf{x}\right)$ has at least one canonical model, because $\Phi$ is well-determined. Then

$$
\begin{aligned}
& P_{1} \mathbf{x} \models_{\Phi} P_{2} \mathbf{x} \\
& \Leftrightarrow \quad \text { Definition of entailments] } \\
& \forall(s, h) \in \text { States. } s, h \models_{\Phi} P_{1} \mathbf{x} \text { implies } s, h \models_{\Phi} P_{2} \mathbf{x}
\end{aligned}
$$

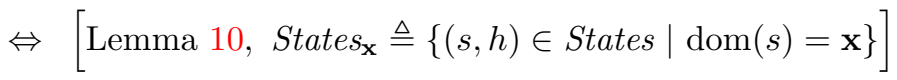

$$
\begin{aligned}
& \forall(s, h) \in \text { States }_{\mathbf{x}} \cdot s, h \models_{\Phi} P_{1} \mathbf{x} \text { implies } s, h \models_{\Phi} P_{2} \mathbf{x} \\
& \Leftrightarrow \quad \text { Lemma 1] } \\
& \forall(s, h) \in \text { States }_{\mathbf{x}} \text {. } \\
& \exists \sigma \in \mathbb{U}_{\Phi}\left(P_{1} \mathbf{x}\right) . s, h \models_{\emptyset} \sigma \\
& \text { implies } \exists \tau \in \mathbb{U}_{\Phi}\left(P_{2} \mathbf{x}\right) . s, h \models_{\emptyset} \tau \\
& \Leftrightarrow[A \rightarrow B \equiv \neg A \vee B] \\
& \forall(s, h) \in \text { States }_{\mathbf{x}} \text {. } \\
& \neg\left(\exists \sigma \in \mathbb{U}_{\Phi}\left(P_{1} \mathbf{x}\right) \cdot s, h \models_{\emptyset} \sigma\right) \\
& \text { or } \exists \tau \in \mathbb{U}_{\Phi}\left(P_{2} \mathbf{x}\right) . s, h \models_{\emptyset} \tau \\
& \Leftrightarrow[\neg \exists x . A \equiv \forall x . \neg A] \\
& \forall(s, h) \in \text { States }_{\mathbf{x}} \text {. } \\
& \forall \sigma \in \mathbb{U}_{\Phi}\left(P_{1} \mathbf{x}\right) . s, h \forall_{\emptyset} \sigma \\
& \text { or } \exists \tau \in \mathbb{U}_{\Phi}\left(P_{2} \mathbf{x}\right) . s, h \models_{\emptyset} \tau \\
& \Leftrightarrow[\forall x \forall y \cdot A \equiv \forall y \forall x \cdot A] \\
& \forall \sigma \in \mathbb{U}_{\Phi}\left(P_{1} \mathbf{x}\right) \cdot \forall(s, h) \in \text { States }_{\mathbf{x}} . \\
& s, h \forall_{\emptyset} \sigma \text { or } \exists \tau \in \mathbb{U}_{\Phi}\left(P_{2} \mathbf{x}\right) . s, h \models_{\emptyset} \tau \\
& \Leftrightarrow[A \rightarrow B \equiv \neg A \vee B] \\
& \forall \sigma \in \mathbb{U}_{\Phi}\left(P_{1} \mathbf{x}\right) \cdot \forall(s, h) \in \text { States }_{\mathbf{x}} . \\
& s, h \models_{\emptyset} \sigma \text { implies } \exists \tau \in \mathbb{U}_{\Phi}\left(P_{2} \mathbf{x}\right) . s, h \models_{\emptyset} \tau \\
& \Leftrightarrow[\forall x . A \rightarrow B \equiv \forall x \in A . B, \text { Def. } 7] \\
& \forall \sigma \in \mathbb{U}_{\Phi}\left(P_{1} \mathbf{x}\right) . \forall(s, h) \in \operatorname{Models}(\sigma) . \exists \tau \in \mathbb{U}_{\Phi}\left(P_{2} \mathbf{x}\right) . s, h \models_{\emptyset} \tau \\
& \Leftrightarrow[\|\operatorname{Models}(\sigma)\|=1 \text { by }(\star) \text { and } \forall x \in\{y\} . A \equiv \exists x \in\{y\} . A] \\
& \forall \sigma \in \mathbb{U}_{\Phi}\left(P_{1} \mathbf{x}\right) . \exists(s, h) \in \operatorname{Models}(\sigma) . \exists \tau \in \mathbb{U}_{\Phi}\left(P_{2} \mathbf{x}\right) . s, h \models_{\emptyset} \tau
\end{aligned}
$$




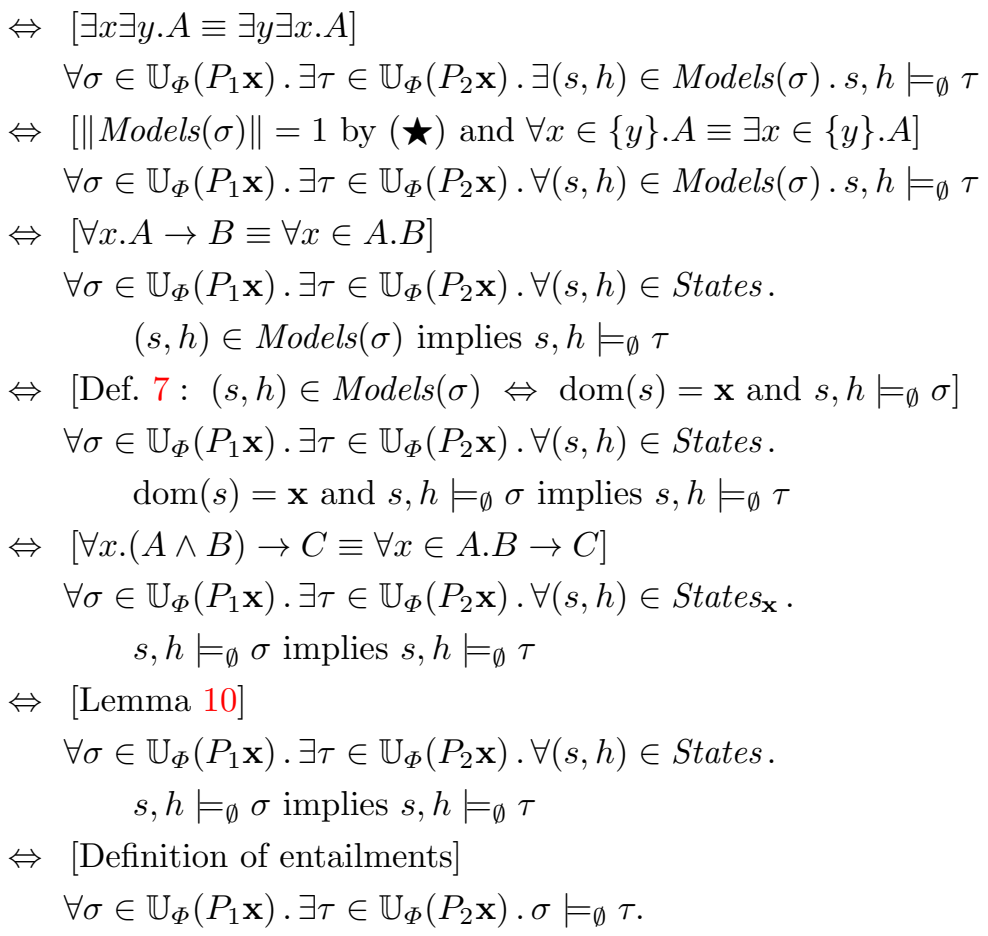

\section{A.21.1 Proof of Lemma 48}

Our previous complexity analysis of Algorithm 1 reveals that $\mathbb{U}_{\Psi}(P \mathbf{x}) \cap L\left(\overline{\mathfrak{A}_{\psi}}\right)=$ $\emptyset$ is decidable in

$$
\mathcal{O}\left(\|\Psi\| \cdot\left\|Q \overline{\mathfrak{A}_{\psi}}\right\|^{M+1} \cdot\left\|\Delta \overline{\mathfrak{A}_{\psi}}\right\|\right) .
$$

Regarding $\|\Psi\|$, applying the Refinement Theorem (Theorem 1) to $\Phi \cup\{P \Leftarrow \varphi\}$ and $\mathfrak{A}_{\mathrm{SAT}}$ (cf. Theorem 3 ) yields an SID $\Psi$ of size

$$
\|\Psi\| \leq c \cdot\|\Phi\| \cdot 2^{\|\varphi\|^{2}} \cdot 2^{2 \alpha^{2}+\alpha} \leq 2^{\text {poly }(k)},
$$

for some positive constant $c$. Then $\Psi$ is computable in $\mathcal{O}\left(2^{\text {poly(k) }}\right)$. Furthermore, $\overline{\mathfrak{A}_{\psi}}$ is obtained from complementation of $\mathfrak{A}_{\psi}$. Thus, by the construction to prove Lemma 2, we obtain that $\left\|Q_{\overline{\mathfrak{A}_{\psi}}}\right\| \leq 2^{\| Q_{\mathfrak{A}_{\psi}}} \|$ and that $\Delta \overline{\mathfrak{A}_{\psi}}$ is decidable

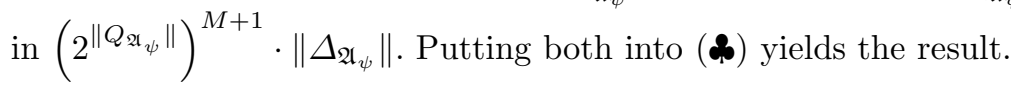

\section{A.21.2 Proof of Lemma 49}

By induction on the structure of symbolic heaps. The base cases are: 
The empty heap emp: In Section A.26, we construct a heap automaton $\mathfrak{A}_{\mathrm{emp}}$ accepting $H_{\mathrm{emp}, \Phi}^{\langle\alpha\rangle}$ with

$$
\left\|Q_{\mathrm{emp}}\right\| \leq 1+(1+\alpha)^{0}=2 \leq 2^{\mathrm{poly}(\alpha)} .
$$

Moreover, $\Delta_{\mathfrak{A}_{\mathrm{emp}}}$ is decidable in polynomial time by Remark 2 .

The points-to assertion $x \mapsto(\mathbf{y})$ : In Section A.26, we construct a heap automaton $\mathfrak{A}_{x \mapsto(\mathbf{y})}$ accepting $H_{x \mapsto(\mathbf{y}), \Phi}^{\langle\alpha\rangle}$ with

$$
\left\|Q_{\mathfrak{A}_{x} \mapsto(\mathbf{y})}\right\| \leq 1+(1+\alpha)^{\|\mathbf{y}\|} \leq 1+(1+\alpha)^{\gamma} \leq 2^{\text {poly }(\alpha)},
$$

because $\|\mathbf{y}\| \leq \gamma$ is considered to be a constant. Moreover, $\Delta_{\mathfrak{A}_{x} \mapsto(\mathbf{y})}$ is decidable in polynomial time by Remark 2 .

The predicate call Px: By assumption, there is nothing to show. Thus, it remains to consider the composite cases:

The separating conjunction $\psi_{1} * \psi_{2}$ : By I.H. there exist heap automata $\mathfrak{A}_{\psi_{1}}$, $\mathfrak{A}_{\psi_{2}} H_{\psi_{1}, \Phi}^{\langle\alpha\rangle}$ and $H_{\psi_{2}, \Phi}^{\langle\alpha\rangle}$ such that $\Delta_{\mathfrak{A}_{\psi_{1}}}$ and $\Delta_{\mathfrak{A}_{\psi_{2}}}$ are decidable in $\mathcal{O}\left(2^{\text {poly }\left(k_{1}\right)}\right)$ and $\mathcal{O}\left(2^{\text {poly }\left(k_{2}\right)}\right)$, where $k_{1}=\|\Phi\|+\left\|\psi_{1}\right\|$ and $k_{2}=\|\Phi\|+\left\|\psi_{2}\right\|$. Moreover, $\left\|Q_{\mathfrak{A}_{\psi_{1}}}\right\| \leq 2^{\text {poly }(\alpha)}$ and $\left\|Q_{\mathfrak{A}_{\psi_{2}}}\right\| \leq 2^{\text {poly }(\alpha)}$, respectively.

In Section A.26, we construct a heap automaton $\mathfrak{A}_{\psi_{1} * \psi_{2}}$ accepting $H_{\psi_{1} * \psi_{2}, \Phi}^{\langle\alpha\rangle}$ with

$$
\begin{aligned}
\left\|Q_{\psi_{1} * \psi_{2}}\right\| & \leq 2^{\alpha} \cdot\left\|Q_{\psi_{1}}\right\| \cdot\left\|Q_{\psi_{2}}\right\| \\
& \leq 2^{\alpha} \cdot 2^{\operatorname{poly}(\alpha)} \cdot 2^{\operatorname{poly}(\alpha)} \\
& =2^{\alpha+\operatorname{poly}(\alpha)+\operatorname{poly}(\alpha)} \\
& \leq 2^{\operatorname{poly}(\alpha)} .
\end{aligned}
$$

Moreover, the time to decide $\Delta_{\mathfrak{A}_{\psi_{1}} * \psi_{2}}$ depends on two factors: First, the number of subformulas occurring in $\psi_{1}, \psi_{2}$ and $\Phi$, which is bounded by $2^{2 \Phi\|+\| \psi_{1}\|+\| \psi_{2} \|} \leq$ $2^{\text {poly }(k)}$. Second, each of these subformulas is applied to $\Delta_{\mathfrak{A}_{\psi_{1}}}$ and $\Delta_{\mathfrak{A}_{\psi_{2}}}$. Thus, $\Delta_{\mathfrak{A}_{\psi_{1} * \psi_{2}}}$ is decidable in $\mathcal{O}\left(2^{\text {poly }(k)} \cdot\left(\left\|\Delta_{\mathfrak{A}_{\psi_{1}}}\right\|+\left\|\Delta_{\mathfrak{A}_{\psi_{2}}}\right\|\right)\right)$. By I.H. this means that $\Delta_{\mathfrak{A}_{\psi_{1} * \psi_{2}}}$ is decidable in

$$
\mathcal{O}\left(2^{\text {poly }(k)} \cdot\left(2^{\text {poly }\left(k_{1}\right)}+2^{\text {poly }\left(k_{2}\right)}\right)\right)=\mathcal{O}\left(2^{\text {poly }(k)}\right) .
$$

The pure formula $\varphi: \Pi$ : By I.H. there exists a heap automaton $\mathfrak{A}_{\varphi}$ accepting $H_{\varphi, \Phi}^{\langle\alpha\rangle}$ such that $\Delta_{\varphi}$ is decidable in $\mathcal{O}\left(2^{\text {poly }\left(k_{1}\right)}\right)$, where $k_{1}=\|\Phi\|+\|\varphi\|$. Moreover, $\left\|Q_{\mathfrak{A}_{\varphi}}\right\| \leq 2^{\text {poly }(\alpha)}$. In Section A.26, we construct a heap automaton $\mathfrak{A}_{\varphi: \Pi}$ accepting $H_{\varphi: \Pi, \Phi}^{\langle\alpha\rangle}$ with

$$
\left\|Q_{\mathfrak{A}_{\varphi}: \Pi}\right\| \leq 2 \cdot\left\|Q_{\mathfrak{A}_{\varphi}}\right\| \leq 2 \cdot 2^{\text {poly }(\alpha)} \leq 2^{\text {poly }(\alpha)} .
$$

Moreover, $\Delta_{\mathfrak{A}_{\varphi}: \Pi \text { II }}$ boils down to deciding $\Delta_{\mathfrak{A}_{\varphi}}$ plus some at most polynomial overhead. Thus, by I.H., $\Delta_{\mathfrak{A}_{\varphi}: \Pi}$ is decidable in $\mathcal{O}\left(2^{\text {poly }(k)}\right)$. 
The existential quantification $\exists z . \varphi$ : By I.H. there exists a heap automaton $\mathfrak{A}_{\varphi}$ accepting $H_{\exists x . \varphi, \Phi}^{\langle\alpha\rangle}$ such that $\Delta_{\mathfrak{A}_{\varphi}}$ is decidable in $\mathcal{O}\left(2^{\text {poly }\left(k_{1}\right)}\right)$, where $k_{1}=$ $\|\Phi\|+\|\varphi\|$. Moreover, $\left\|Q_{\mathfrak{A}_{\varphi}}\right\| \leq 2^{\text {poly }(\alpha)}$.

In Section A.26, we construct a heap automaton $\mathfrak{A}_{\exists z . \varphi}$ accepting $H_{\exists x . \varphi, \Phi}^{\langle\alpha\rangle}$ with

$$
\begin{aligned}
\left\|Q_{\mathfrak{A}_{\exists z \cdot \varphi}}\right\| & \leq(\alpha+1) \cdot\left\|Q_{\mathfrak{A}_{\varphi}}\right\| \cdot 2^{2 \alpha^{2}+\alpha} \\
& \leq(\alpha+1) \cdot 2^{\text {poly }(\alpha)} \cdot 2^{2 \alpha^{2}+\alpha} \\
& \leq 2^{\text {poly }(\alpha)} .
\end{aligned}
$$

Moreover, $\Delta_{\mathfrak{A}_{\exists z . \varphi}}$ boils down to deciding $\Delta_{\mathfrak{A}_{\varphi}}$ plus some overhead which is at most polynomial due to Remark 2. Thus, by I.H., $\Delta_{\mathfrak{A}_{\exists z . \varphi}}$ is decidable in $\mathcal{O}\left(2^{\text {poly }(k)}\right)$.

\section{A.22 Omitted calculations in proof of Theorem 50}

$$
\begin{aligned}
& \mathcal{O}\left(\|\Psi\| \cdot\left\|Q \overline{\overline{\mathfrak{A}}_{\psi}}\right\|^{M+1} \cdot\left\|\Delta \overline{\overline{\mathfrak{A}}_{\psi}}\right\|\right) \\
& =\left[\|\Psi\| \leq 2^{\text {poly }(k)},\left\|Q_{\overline{\mathfrak{A}_{\psi}}}\right\| \leq 2^{\left\|Q_{\mathfrak{A}_{\psi}}\right\|}, M \leq 2 k\right] \\
& \mathcal{O}\left(2^{\text {poly }(k)} \cdot\left(2^{\left\|Q_{\mathfrak{A}_{\psi}}\right\|}\right)^{2 k} \cdot\left\|\Delta_{\overline{\mathfrak{A}_{\psi}}}\right\|\right) \\
& =\left[\left\|\Delta_{\overline{\mathfrak{A}}_{\psi}}\right\| \leq\left(2^{\left\|Q_{\mathfrak{A}_{\psi}}\right\|}\right)^{M+1} \cdot\left\|\Delta_{\mathfrak{A}_{\psi}}\right\|\right] \\
& \mathcal{O}\left(2^{\text {poly }(k)} \cdot\left(2^{\left\|Q_{\mathfrak{A}_{\psi}}\right\|}\right)^{4 k} \cdot\left\|\Delta_{\mathfrak{A}_{\psi}}\right\|\right) \\
& =\left[\left\|Q_{\mathfrak{A}_{\psi}}\right\| \leq 2^{\text {poly }(k)}\right] \\
& \mathcal{O}\left(2^{\text {poly }(k)} \cdot\left(2^{2^{\text {poly }(k)}}\right)^{4 k} \cdot\left\|\Delta_{\mathfrak{A}_{\psi}}\right\|\right) \\
& =\left[\left(a^{b}\right)^{c}=a^{b c},\left\|\Delta_{\mathfrak{A}_{\psi}}\right\| \in \mathcal{O}\left(2^{\text {poly }(k)}\right)\right] \\
& \mathcal{O}\left(2^{\text {poly }(k)} \cdot 2^{4 k \cdot 2^{\text {poly }(k)}} \cdot 2^{\text {poly }(k)}\right) \\
& =\mathcal{O}\left(2^{2^{\text {poly }(k)}}\right)
\end{aligned}
$$

\section{A.23 Proof of Lemma 51}

By Lemma $49,\left\|Q_{\mathfrak{A}_{\psi}}\right\| \leq 2^{\text {poly }(\alpha)}$ and $\Delta_{\mathfrak{A}_{\psi}}$ is decidable in $\mathcal{O}\left(2^{\text {poly }(k)}\right)$. Since $\alpha$ is bounded by a constant, so is $\left\|Q_{\mathfrak{A}_{\psi}}\right\|$. Then, by Lemma 48, SL-ENTAIL $\operatorname{Si}_{\langle\alpha\rangle}^{\Phi}$ is decidable in

$$
\mathcal{O}\left(2^{\text {poly }(k)} \cdot\left(2^{\left\|Q_{\mathfrak{A}_{\psi}}\right\|}\right)^{2(M+1)} \cdot 2^{\text {poly }(k)}\right)=\mathcal{O}\left(2^{\text {poly }(k)}\right),
$$


which clearly is in ExPTime.

\section{A.24 Lower bound for entailments}

The proof of the ExpTime-lower bound in [1] is by reducing the inclusion problem for nondeterministic finite tree automata (NFTA, cf. [18]) to the entailment problem. Their proof requires a constant (or free variable) for each symbol in the tree automatons alphabet. In contrast, we prove their result by encoding the alphabet in a null-terminated singly-linked list. Thus, a tree $a(b, a(b, b)$ is encoded by a reduced symbolic heap

$$
\begin{aligned}
& \exists z_{1} z_{2} z_{3} z_{4} z_{5} z_{6} z_{7} \\
& \quad x \mapsto\left(z_{1} z_{2} \text { null }\right) \\
& \quad * z_{1} \mapsto\left(\text { null null } z_{3}\right) * z_{3} \mapsto(\text { null null null }) \\
& \quad * z_{2} \mapsto\left(z_{4} z_{5} \text { null }\right) \\
& \quad * z_{4} \mapsto\left(\text { null null } z_{6}\right) * z_{6} \mapsto(\text { null null null }) \\
& \quad * z_{5} \mapsto\left(\text { null null } z_{7}\right) * z_{7} \mapsto(\text { null null null }),
\end{aligned}
$$

where the symbol $a$ is encoded by having null as third component in a points-to assertion and symbol $b$ by a null terminated list of length one.

Now, given some NFTA $\mathfrak{T}=(Q, \Sigma, \Delta, F)$ with $\Sigma=\left\{a_{1}, \ldots, a_{n}\right\}$, we construct a corresponding $\Phi$. Without less of generality, we assume that $\mathfrak{T}$ contains no unreachable or unproductive states. We set $\operatorname{Pred}(\Phi) \triangleq Q \cup \Sigma \cup\{I\}$, where each predicate symbol is of arity one. Then, for each symbol $a_{i} \in \Sigma$ one rule of the form $a_{1} \Leftarrow \mathbf{x}_{0}[1]=$ null or, for $1<i \leq n$,

$$
\begin{aligned}
& a_{i} \Leftarrow \exists z_{1} z_{2} \ldots z_{i-1} \cdot \mathbf{x}_{0} 1[\mapsto]\left(\text { null null } z_{1}\right) \\
& * \star_{1 \leq j<i} z_{j} \mapsto\left(\text { null null } z_{j+1}\right):\left\{z_{i-1}=\text { null }\right\}
\end{aligned}
$$

is added to $\Phi$. Furthermore, for each $\left(p_{1} \ldots p_{m}, a_{i}, p_{0}\right) \in \Delta, 1 \leq i \leq n$, we add a rule

$$
\begin{array}{r}
p_{0} \Leftarrow \exists z_{1} \ldots z_{m+1} \cdot \mathbf{x}_{0}[1] \mapsto\left(z_{1} \ldots z_{m+1}\right) \\
* a_{i}\left(z_{m+1}\right) * \star_{1 \leq i \leq m} p_{i}\left(z_{i}\right) .
\end{array}
$$

Finally, we add rules $I \Leftarrow p \mathbf{x}_{0}[1]:\left\{\mathbf{x}_{0}[1] \neq\right.$ null $\}$ for each $p \in F$. Clearly $\Phi$ is established. Moreover, it is easy to verify that, given two NFTAs $\mathfrak{T}_{1}$ and $\mathfrak{T}_{2}$ with distinct sets of states, we have

$$
I_{1} x \models_{\Phi_{1} \cup \Phi_{2}} I_{2} x \text { iff } L\left(\mathfrak{T}_{1}\right) \subseteq L\left(\mathfrak{T}_{2}\right) .
$$

Thus, following [1], if $H_{I x, \Phi}^{\langle 1\rangle}$ can be accepted by a heap automaton, the entailment problem SL-ENTAIL ${ }_{\Phi}^{\langle\alpha\rangle}$ is ExPTIME-hard for certain SIDs $\Phi$ fixed $\alpha=1$, and a fixed arity of points-to assertions $\gamma=3$. Such a heap automaton can 
easily be constructed. Formally, let $\mathfrak{T}=(Q, \Sigma, \Delta, F)$ be an NFTA as above and $Q=\left\{p_{1}, \ldots, p_{k}\right\}$ for some $k>0$. Furthermore, for each state $p_{i}$, let $t_{i}$ be some fixed finite tree that is accepted by the tree automaton $\mathfrak{T}_{i}=\left(Q, \Sigma, \Delta,\left\{p_{i}\right\}\right)$ and $\tau_{i}$ be the corresponding encoding as a reduced symbolic heap. One possible (not necessarily efficient) heap automaton $\mathfrak{A}=\left(Q_{\mathfrak{A}}, \mathrm{SH}_{\langle 1\rangle}, \Delta_{\mathfrak{A}}, F_{\mathfrak{A}}\right)$ is given by:

$$
\begin{aligned}
& Q_{\mathfrak{A}} \triangleq\left\{\tau_{i} \mid 1 \leq i \leq k\right\} \cup\left\{a_{i} \mid 1 \leq i \leq n\right\} \\
& F_{\mathfrak{A}} \triangleq F \\
& q_{0} \stackrel{\varphi}{\rightarrow} \mathfrak{A} q_{1} \ldots q_{m} \text { iff } \varphi\left[P_{1} / q_{1}, \ldots, P_{m} / q_{m}\right] \models_{\Phi} P \mathbf{x}_{0}[1],
\end{aligned}
$$

where each $a_{i}$ corresponds to the reduced symbolic heap encoding symbol $a_{i}$ and $P$ is the predicate $p_{i}$ corresponding to reduced symbolic heap $\tau_{i}$ as previously described.

\section{A.25 Constructing Well-Determined SIDs}

In this section, we show that an established SID - and analogously an established symbolic heap - can be automatically transformed into a well-determined one. Intuitively, the main difference between an established symbolic heap $\varphi$ and a determined one $\psi$ lies in their free variables: Since each existentially quantified variable of $\varphi$ is eventually allocated or equal to a free variable, all variables apart from free variables are definitely unequal in $\varphi$. The same holds for $\psi$, but each free variable is additionally either definitely equal or definitely unequal to any other variable of $\psi$ (cf. Section 6). Thus, the main idea to transform $\varphi$ into a determined symbolic heap is to add explicit pure formulas between each free variable and each other variable.

More formally, let $\operatorname{Cmp}(\mathbf{y}, \varphi)$ be the set of all sets $\Pi$ consisting of exactly one pure formula $x \sim y$ for each $x \in \operatorname{Var}(\varphi)$ and each $y \in \mathbf{y}$, where $\varphi \in \mathrm{SH}$ and $\mathbf{y}$ is some tuple of variables. Then, the following construction is essential to transform symbolic heaps into determined ones.

Definition 22. Let $\varphi=\exists \mathbf{z} . \Sigma * \Gamma: \Pi$ be an established symbolic heap with $\left\|\Gamma^{\varphi}\right\|=m$. Moreover, let $\mathbf{y}$ be a tuple of variables of length $k \geq 0$ and $\Lambda \in$ $\operatorname{Cmp}(\mathbf{y}, \varphi)$. Then,

$$
\begin{aligned}
\operatorname{det}(\varphi, \mathbf{y}, \Lambda) & \triangleq \exists \mathbf{z} \cdot \Sigma * \operatorname{propagate}(\varphi, \mathbf{y}): \Pi \cup \Lambda, \\
\operatorname{propagate}(\varphi, \mathbf{y}) & \triangleq\left(P_{1}, k\right) \mathbf{x}_{1} \mathbf{y} * \ldots\left(P_{m}, k\right) \mathbf{x}_{m} \mathbf{y},
\end{aligned}
$$

where $\left(P_{i}^{\varphi}, \beta\right)$ is a predicate symbol of arity $\operatorname{ar}\left(P_{i}^{\varphi}\right)+\beta$.

Example 10. Let $\varphi$ denote the symbolic heap in the lower rule of predicate sll in Example 1. Then $\operatorname{det}\left(\varphi, \mathbf{x}_{0}^{\varphi}, \emptyset\right)$ is given by

$$
\exists z \cdot \mathbf{x}_{0}[1] \mapsto(z) *(\mathbf{s l l}, 2) z \mathbf{x}_{0}[2] \mathbf{x}_{0}^{\varphi}:\left\{\mathbf{x}_{0}[1] \neq \mathbf{x}_{0}[2]\right\} .
$$

Lemma 37. Let $\Phi \in$ SID be established. Then one can construct an SID $\Psi$ with $\operatorname{Pred}(\Phi) \subseteq \operatorname{Pred}(\Psi)$ such that for each $P \in \operatorname{Pred}(\Phi)$, we have: 
- $\tau$ is well-determined for each $\tau \in \mathbb{U}_{\Psi}\left(P \mathbf{x}_{0}\right)$,

$-\tau \models_{\Phi} P \mathbf{x}_{0}$ holds for each $\tau \in \mathbb{U}_{\Psi}\left(P \mathbf{x}_{0}\right)$, and

$-\tau \models_{\Psi} P \mathbf{x}_{0}$ holds for each $\tau \in \mathbb{U}_{\Phi}\left(P \mathbf{x}_{0}\right)$.

Here, the last two points ensure that models of predicate calls remain unchanged, although their actual unfoldings might differ.

Proof. We first construct a determined SID $\Omega$. For each $Y, Z \in \operatorname{Pred}(\Phi)$, we introduce a fresh predicate symbol $(Y, \operatorname{ar}(Z))$, i.e.

$$
\operatorname{Pred}(\Omega) \triangleq \operatorname{Pred}(\Phi) \cup\{(Y, \operatorname{ar}(Z)) \mid Y, Z \in \operatorname{Pred}(\Phi)\} .
$$

Furthermore, for each arity $k$ of some predicate symbol in $\Phi, \Omega$ contains two kinds of rules: First, there is a rule that propagates the free variables of a symbolic heap through all of its predicate calls while comparing it to every variable. Formally, we add a rule $P \Leftarrow \operatorname{det}\left(\varphi, \mathrm{x}_{0}^{\varphi}, \Lambda\right)$ for each $P \Leftarrow \varphi \in \Phi$ and each $\Lambda \in \operatorname{Cmp}\left(\mathrm{x}_{0}^{\varphi}, \varphi\right)$. The second kind of rule continues the propagation and comparison of a selected tuple of variables initiated by the first rule. Formally, we add a rule $(P, k) \Leftarrow$ $\operatorname{det}(\varphi, \mathbf{y}, \Lambda)$, where $\mathbf{y}$ is a tuple of $k$ fresh variables, for each $P \Leftarrow \varphi \in \Phi$ and each $\Lambda \in \operatorname{Cmp}(\mathbf{y}, \varphi)$. Then $\Omega$ is determined.

To obtain a well-determined SID, we apply the Refinement Theorem (Theorem 1) to $\Omega$ and the heap automaton $\mathfrak{A}_{\mathrm{SAT}}$ (cf. Theorem 3 ) accepting all satisfiable reduced symbolic heaps. This yields the desired SID $\Psi$ in which every unfolding of each predicate call is satisfiable and determined.

It remains to prove that our construction of $\Psi$ is correct.

$\tau$ is well-determined for each $\tau \in \mathbb{U}_{\Psi}\left(P \mathbf{x}_{0}\right)$. Let $\tau \in \mathbb{U}_{\Psi}\left(P \mathbf{x}_{0}\right)$. Since each rule in $\Psi$ is obtained from $\Phi$ by adding pure formulas and free variables only and $\Phi$ is established, $\Psi$ is established as well. Then models of $\tau$ differ in the interpretation of free variables only. By construction of $\Psi$ we know that for each free variable $x \in \mathbf{x}_{0}$ and each variable $y \in \operatorname{Var}(\tau)$ there exists either an equality $x=y$ or an inequality $x \neq y$ in $\Pi^{\tau}$. Hence, each variable is either allocated or definitely equal or unequal to each other variable by a pure formula. Then $\tau$ is determined (cf. Section 6). Furthermore, by Theorem 3, the Refinement Theorem ensures that every unfolding of each predicate symbol is satisfiable without changing the unfoldings themselves. Thus $\Psi$ is well-determined.

$\tau \models_{\Psi} P \mathbf{x}_{0}$ for each $\tau \in \mathbb{U}_{\Phi}\left(P \mathbf{x}_{0}\right)$. Let $\tau \in \mathbb{U}_{\Phi}\left(P \mathbf{x}_{0}\right)$. By construction of $\Omega$, we have $\sigma=\operatorname{det}\left(\tau, \mathbf{x}_{0}^{\tau}, \Lambda\right) \in \mathbb{U}_{\Psi}\left(P \mathbf{x}_{0}\right)$ for each $\Lambda \in \operatorname{Cmp}\left(\mathbf{x}_{0}^{\tau}, \tau\right)$ such that $\sigma$ is satisfiable. Then, for each stack-heap pair $s, h$, we have

$$
\begin{aligned}
& s, h \models_{\emptyset} \tau \\
\Rightarrow & {\left[\operatorname{set} \Lambda \triangleq\left\{y \sim \mathbf{x}_{0}^{\tau} \mid s(y) \sim s\left(\mathbf{x}_{0}^{\tau}\right)\right\}\right] } \\
& s, h \models_{\emptyset} \tau \wedge s, h \models_{\emptyset} \Lambda \\
\Rightarrow & {\left[\text { Definition of } \sigma=\operatorname{det}\left(\tau, \mathbf{x}_{0}^{\tau}, \Lambda\right) \in \mathbb{U}_{\Psi}\left(P \mathbf{x}_{0}\right)\right] } \\
& \exists \sigma \in \mathbb{U}_{\Psi}\left(P \mathbf{x}_{0}\right) \cdot s, h \models_{\emptyset} \sigma
\end{aligned}
$$




$$
\begin{gathered}
\Rightarrow \quad[\text { SL semantics }] \\
s, h \models_{\Psi} P \mathbf{x}_{0} .
\end{gathered}
$$

Hence, $\tau \models_{\Psi} P \mathbf{x}_{0}$.

$\tau \models_{\Phi} P \mathbf{x}_{0}$ for each $\tau \in \mathbb{U}_{\Psi}\left(P \mathbf{x}_{0}\right)$. Let $\tau \in \mathbb{U}_{\Psi}\left(P \mathbf{x}_{0}\right)$. By construction of $\Psi$, there exists $\sigma \in \mathbb{U}_{\Phi}\left(P \mathbf{x}_{0}\right)$ and $\Lambda \in \operatorname{Cmp}\left(\mathbf{x}_{0}^{\sigma}, \sigma\right)$ such that $\tau=\operatorname{det}\left(\sigma, \mathbf{x}_{0}^{\sigma}, \Lambda\right)$. Then, for each stack-heap pair $s, h$, we have

$$
\begin{aligned}
& s, h \models_{\emptyset} \tau \\
\Rightarrow & {\left[\text { Definition of } \tau=\operatorname{det}\left(\sigma, \mathbf{x}_{0}^{\sigma}, A\right)\right] } \\
& s, h \models_{\emptyset} \sigma \text { and } s, h \models_{\emptyset} \Lambda \\
\Rightarrow & {[A \wedge B \rightarrow A] } \\
& s, h \models_{\emptyset} \sigma \\
\Rightarrow & {\left[\sigma \in \mathbb{U}_{\Phi}\left(P \mathbf{x}_{0}\right)\right] } \\
& \exists \sigma \in \mathbb{U}_{\Phi}\left(P \mathbf{x}_{0}\right) \cdot s, h \models_{\emptyset} \sigma \\
\Rightarrow & {[\text { SL semantics }] } \\
& s, h \models_{\Phi} P \mathbf{x}_{0} .
\end{aligned}
$$

Hence, $\tau \models_{\Phi} P \mathbf{x}_{0}$

\section{A.26 Proof of Theorem 10}

Recall from Definition 12 the set of all reduced symbolic heaps in $\mathcal{C}$ (over the same free variables as $\varphi$ ) entailing an unfolding of $\varphi$ :

$$
H_{\varphi, \Phi}^{\mathcal{C}} \triangleq\left\{\sigma \in \operatorname{RSH}_{\mathcal{C}} \mid\left\|\mathbf{x}_{0}^{\sigma}\right\|=\left\|\mathbf{x}_{0}^{\varphi}\right\| \text { and } \exists \tau \in \mathbb{U}_{\Phi}(\varphi) . \sigma \models_{\emptyset} \tau\right\}
$$

Theorem 10. Let $\alpha \in \mathbb{N}$ and $\Phi \in \operatorname{SID}_{F V \leq \alpha}$ be established. Moreover, for each predicate symbol $P \in \operatorname{Pred}(\Phi)$, let there be a heap automaton over $\mathrm{SH}_{\langle\alpha\rangle}$ accepting $H_{P \mathbf{x}, \Phi}^{\langle\alpha\rangle}$. Then, for every well-determined symbolic heap $\varphi \in \mathrm{SH}^{\Phi}$, there is a heap automaton over $\mathrm{SH}_{\langle\alpha\rangle}$ accepting $H_{\varphi, \Phi}^{\langle\alpha\rangle}$.

The proof is by induction on the syntax of symbolic heaps. Since each case requires the construction of a suitable heap automaton and a corresponding correctness proof, we split the proof across multiple lemmas, which are shown subsequently in the remainder of this section. Furthermore, we make some preliminary remarks to reduce the technical effort.

Remark 4. We assume without loss of generality that every predicate call occurring in a symbolic heap $\varphi \in \mathrm{SH}_{\langle\alpha\rangle}$ is annotated with a state of the tracking automaton $\mathfrak{A}_{\text {TRACK }}$ introduced in Definition 10 . Thus, for every predicate call $P_{i}^{\varphi} \mathbf{x}_{i}^{\varphi}$, we assume the availability of 
- a set $A_{i} \subseteq \mathbf{x}_{i}^{\varphi}$ capturing all allocated variables in a (fixed) unfolding of $P_{i}^{\varphi} \mathbf{x}_{i}^{\varphi}$, and

- a finite set of pure formulas $\Pi_{i}$ over $\mathbf{x}_{i}^{\varphi}$ capturing all definite equalities and inequalities between variables in $\mathbf{x}_{i}^{\varphi}$.

This assumption is an optimization that prevents us from applying Lemma 3 again and again to keep track of the aforementioned relationships between parameters of predicate calls.

Remark 5. Furthermore, by Definition 13, every predicate call has at most $\alpha$ parameters. However, the number of free variables of the symbolic heap itself, i.e. the formula at the root of an unfolding tree, is not necessarily bounded by $\alpha$. To avoid additional case distinctions in correctness proofs, we present constructions where the number of free variables is bounded by $\alpha$ as well. In each of our constructions, this corner case can be dealt with by adding one dedicated final state $q_{>\alpha}$ such that $\left(\varepsilon, \tau, q_{>\alpha}\right) \in \Delta$ if and only if the number of free variables $\left\|\mathbf{x}_{0}^{\tau}\right\|$ is greater than $\alpha$ and the property in question is satisfied by $\tau$. General symbolic heaps with more than $\alpha$ free variables are treated analogously. Once the final state $q_{>\alpha}$ has been reached no further transition is possible. Thus, only symbolic heaps at the root of an unfolding tree may enter $q_{>\alpha}$.

\section{Proof (Proof of Theorem 10).}

The remainder of the proof is summarized as follows:

- The base cases $x \mapsto(\mathbf{y})$ and emp are shown in Section A.26 and Section A.26, respectively.

- For predicate calls $P \mathbf{x}_{0}$, we already know by assumption that $H_{P \mathbf{x}, \Phi}^{\langle\alpha\rangle}$ is accepted by a heap automaton over $\mathrm{SH}_{\langle\alpha\rangle}$.

- Section A.26 shows that $H_{\varphi * \psi, \Phi}^{\langle\alpha\rangle}$ is accepted by a heap automaton over $\mathrm{SH}_{\langle\alpha\rangle}$ if $\varphi, \psi$ are defined over the same set of free variables. This is sufficient, because we do not require that these variables actually occur in both symbolic heaps.

- Section A.26 shows that $H_{\varphi: \Pi, \Phi}^{\langle\alpha\rangle}$ is accepted by a heap automaton over $\mathrm{SH}_{\langle\alpha\rangle}$, where $\Pi$ is a finite set of pure formulas over the free variables of $\varphi$.

- Section A.26 shows that $H_{\exists z, \varphi, \Phi}^{\langle\alpha\rangle}$ is accepted by a heap automaton over $\mathrm{SH}_{\langle\alpha\rangle}$, where $z$ is a single variable. Repeated application then completes the proof.

Hence, for each symbolic heap $\varphi \in \mathrm{SH}_{\langle\alpha\rangle}^{\Phi}$, a corresponding heap automaton over $\langle\alpha\rangle$ accepting $H_{\varphi, \Phi}^{\langle\alpha\rangle}$ can be constructed.

The Points-to Assertion Let $\mathbf{u} \in U_{n}=\{1, \ldots, \alpha\} \times\{0,1, \ldots, \alpha\}^{n-1}$ for some fixed natural number $n \geq 1$. Moreover, we associate the points-to assertion

$$
\sigma_{\mathbf{u}} \triangleq \mathbf{x}_{0}[\mathbf{u}[1]] \mapsto\left(\mathbf{x}_{0}[\mathbf{u}[2]] \ldots \mathbf{x}_{0}[\mathbf{u}[n]]\right)
$$


with each tuple $\mathbf{u} \in U_{n} .{ }^{16}$ We construct a heap automaton $\mathfrak{A}_{\sigma_{\mathbf{v}}}=\left(Q, \mathrm{SH}_{\langle\alpha\rangle}, \Delta, F\right)$ accepting $H_{\sigma_{\mathbf{v}}, \Phi}^{\langle\alpha\rangle}$ for some fixed tuple $\mathbf{v} \in U_{n}$ as follows:

$$
Q \triangleq\left\{\sigma_{\mathbf{u}} \mid \mathbf{u} \in U_{n}\right\} \cup\{\mathrm{emp}\} \quad F \triangleq\left\{\sigma_{\mathbf{v}}\right\}
$$

Moreover, for $\varphi \in \mathrm{SH}_{\langle\alpha\rangle}$ with $\left\|\Gamma^{\varphi}\right\|=m$, the transition relation $\Delta$ is given by:

$$
q_{1} \ldots q_{m} \stackrel{\varphi}{\rightarrow} \mathfrak{A}_{\sigma_{\mathbf{v}}} q_{0} \quad \text { iff } \quad \operatorname{reduce}\left(\varphi, q_{1} \ldots q_{m}\right) \models_{\emptyset} q_{0}
$$

where $\operatorname{reduce}\left(\varphi, q_{1} \ldots q_{m}\right) \triangleq \varphi\left[P_{1} / q_{1}: \Pi_{i}, \ldots, P_{m} / q_{m}: \Pi_{m}\right]$ and each set $\Pi_{i}$ denotes the set of pure formulas obtained from the tracking automaton (see Definition 8), which is assumed to be readily available by Remark 4 .

Lemma 38. $\mathfrak{A}_{\sigma_{\mathrm{v}}}$ satisfies the compositionality property.

Proof. Let $\varphi \in \mathrm{SH}_{\langle\alpha\rangle}$ with $\left\|\Gamma^{\varphi}\right\|=m$. Moreover, for each $1 \leq i \leq m$, let $\tau_{i} \in \mathrm{RSH}_{\langle\alpha\rangle}$ and $\tau \triangleq \varphi\left[P_{1}^{\varphi} / \tau_{1}, \ldots, P_{m}^{\varphi} / \tau_{m}\right]$. Assume $\varepsilon \stackrel{\tau}{\rightarrow}_{\mathfrak{A}_{\sigma_{\mathbf{v}}}} q_{0}$. Three cases arise depending on the value of $q_{0} \in Q$.

Case 1: $q_{0}=\mathrm{emp}$

$$
\begin{aligned}
& \varepsilon \stackrel{\tau}{\rightarrow}_{\mathfrak{A}_{\sigma_{\mathrm{v}}}} \text { emp } \\
\Leftrightarrow & {\left[\text { Definition of } \Delta,\left\|\Gamma^{\tau}\right\|=0\right] } \\
& \tau \models_{\Phi} \text { emp } \\
\Leftrightarrow & {\left[\text { choose } q_{1}=\ldots=q_{m}=\mathrm{emp}\right] } \\
& \bigwedge_{1 \leq i \leq m} q_{i}=\text { emp and } \tau \models_{\Phi} \text { emp } \\
\Leftrightarrow & {\left[\tau=\varphi\left[P_{1}^{\varphi} / \tau_{1}, \ldots, P_{m}^{\varphi} / \tau_{m}\right] \text { contains no points-to assertion }\right] } \\
& \bigwedge_{1 \leq i \leq m} q_{i}=\text { emp and } \varphi \models_{\Phi} \text { emp } \\
& \quad \text { and } \forall 1 \leq i \leq m \cdot \tau_{i} \models_{\Phi} \text { emp } \\
\Leftrightarrow & {[\text { Definition of } \Delta] } \\
& \exists q_{1}, \ldots, q_{m} \in Q \cdot q_{1} \ldots q_{m} \stackrel{\varphi}{\rightarrow}_{\mathfrak{A}_{\sigma \mathrm{V}}} q_{0} \\
& \quad \text { and } \forall 1 \leq i \leq m . \varepsilon{\stackrel{\tau_{i}}{\rightarrow}}_{\mathfrak{A}_{\sigma_{\mathrm{V}}}} q_{i} .
\end{aligned}
$$

Case 2: $q_{0}=\sigma_{\mathbf{u}}$

$$
\begin{aligned}
& \varepsilon \stackrel{\tau}{\rightarrow}_{\mathfrak{A}_{\sigma_{\mathbf{v}}}} \sigma_{\mathbf{u}} \\
\Leftrightarrow & {\left[\text { Definition of } \Delta,\left\|\Gamma^{\tau}\right\|=0\right] } \\
& \tau \models_{\Phi} \sigma_{\mathbf{u}}
\end{aligned}
$$

Now, $\tau \models_{\Phi} \sigma_{\mathbf{u}}$ holds if and only if $\tau$ contains at most one points-to assertion $\sigma_{\mathbf{w}}$, $\mathbf{w} \in U_{n}$, where each variable is free. If this points-to assertion is contained in $\varphi$

$\overline{{ }^{16} \text { Recall that }} \mathbf{x}_{0}[0]=$ null. 
then choose $q_{1}=\ldots=q_{m}=$ emp. Otherwise, if exactly $\tau_{k}, 1 \leq k \leq m$ contains a points-to assertion, choose $q_{k}=\sigma_{\mathbf{w}}$ and $q_{i}=\operatorname{emp}$ for each $i \neq k$. With these choices, our computation continues as follows:

$$
\begin{aligned}
(\dagger) \Leftrightarrow & {\left[\text { choice of } q_{1}, \ldots, q_{m}\right] } \\
& \exists q_{1}, \ldots, q_{m} \in Q \cdot \tau \models_{\Phi} \sigma_{\mathbf{u}} \\
& \quad \text { and } \forall 1 \leq i \leq m . \tau_{i} \models_{\Phi} q_{i} \\
\Leftrightarrow & {[\text { Definition of } \Delta] } \\
& \exists q_{1}, \ldots, q_{m} \in Q \cdot \tau \models_{\Phi} \sigma_{\mathbf{u}} \\
& \quad \text { and } \forall 1 \leq i \leq m \cdot \varepsilon \stackrel{\tau_{i}}{\rightarrow} \mathfrak{A}_{\sigma_{\mathbf{v}}} q_{i}
\end{aligned}
$$

Since each variable of points-to assertion $\sigma_{\mathbf{w}}$ is equal to a free variable and, by Lemma 3, two free variables are equal in $\tau$ if and only if they are equal in $\operatorname{reduce}\left(\varphi, q_{1} \ldots q_{m}\right)$, we have $\operatorname{reduce}\left(\varphi, q_{1} \ldots q_{m}\right) \models_{\Phi} \sigma_{\mathbf{u}}$ if and only if $\tau \models_{\varphi} \sigma_{\mathbf{u}}$. Thus:

$$
\begin{array}{cl}
\Leftrightarrow & {\left[\operatorname{reduce}\left(\varphi, q_{1} \ldots q_{m}\right) \models_{\Phi} \sigma_{\mathbf{u}} \text { iff } \tau \models_{\varphi} \sigma_{\mathbf{u}}\right]} \\
& \exists q_{1}, \ldots, q_{m} \in Q \cdot \operatorname{reduce}\left(\varphi, q_{1} \ldots q_{m}\right) \models_{\Phi} \sigma_{\mathbf{u}} \\
& \text { and } \forall 1 \leq i \leq m \cdot \varepsilon{\stackrel{\tau_{i}}{\rightarrow}}_{\mathfrak{A}_{\sigma_{\mathbf{v}}}} q_{i} \\
\Leftrightarrow \quad & {[\text { Definition of } \Delta]} \\
& \exists q_{1}, \ldots, q_{m} \in Q \cdot q_{1} \ldots q_{m} \stackrel{\varphi}{\rightarrow}_{\mathfrak{A}_{\sigma \mathbf{v}}} q_{0} \\
\text { and } \forall 1 \leq i \leq m \cdot \varepsilon{\stackrel{\tau_{i}}{\rightarrow}}_{\mathfrak{A}_{\sigma_{\mathbf{v}}}} q_{i} .
\end{array}
$$

Lemma 39. $L\left(\mathfrak{A}_{\sigma_{\mathbf{v}}}\right)=H_{\sigma_{\mathbf{v}}, \Phi}^{\langle\alpha\rangle}$.

Proof. Let $\tau \in \mathrm{RSH}_{\langle\alpha\rangle}$. Then

$$
\begin{aligned}
& \tau \in L\left(\mathfrak{A}_{\sigma_{\mathrm{v}}}\right) \\
& \left.\Leftrightarrow \quad \text { [Definition of } L\left(\mathfrak{A}_{\sigma_{\mathrm{v}}}\right)\right] \\
& \exists q \in F . \varepsilon \stackrel{\tau}{\rightarrow}_{\mathfrak{A}_{\sigma_{\mathbf{v}}}} q \\
& \Leftrightarrow \quad \text { Definition of } F] \\
& \varepsilon \stackrel{\tau}{\rightarrow} \mathfrak{A}_{\sigma_{\mathbf{v}}} \sigma_{\mathbf{v}} \\
& \Leftrightarrow \quad \text { Definition of } \Delta] \\
& \operatorname{reduce}(\varphi, \varepsilon) \models_{\emptyset} \sigma_{\mathbf{v}} \\
& \Leftrightarrow\left[\left\|\Gamma^{\tau}\right\|=0 \text { implies } \tau=\operatorname{reduce}(\varphi, \varepsilon)\right] \\
& \tau \models_{\emptyset} \sigma_{\mathbf{v}} \\
& \Leftrightarrow \quad\left[\text { Definition of } H_{\sigma_{\mathbf{v}}, \Phi}^{\langle\alpha\rangle}\right] \\
& \tau \in H_{\sigma_{\mathbf{v}}, \Phi}^{\langle\alpha\rangle} .
\end{aligned}
$$


The Empty Heap A heap automaton $\mathfrak{A}_{\mathrm{emp}}$ accepting $H_{\mathrm{emp}, \Phi}^{\langle\alpha\rangle}$ is constructed analogously to $\mathfrak{A}_{\sigma_{\mathbf{v}}}$, the heap automaton constructed for the points-to assertion in Section A.26. The only exception is that $F_{\mathfrak{A}_{\mathrm{emp}}} \triangleq\{\mathrm{emp}\}$ is chosen as set of final states. ${ }^{17}$ Since the compositionality property for this automaton already has been shown in Lemma 38 , it suffices to show that $\mathfrak{A}_{\text {emp }}$ accepts the correct language.

Lemma 40. $L\left(\mathfrak{A}_{\mathrm{emp}}\right)=H_{\mathrm{emp}, \Phi}^{\langle\alpha\rangle}$.

Proof. Let $\tau \in \mathrm{RSH}_{\langle\alpha\rangle}$. Then

$$
\begin{aligned}
& \tau \in L\left(\mathfrak{A}_{\mathrm{emp}}\right) \\
\Leftrightarrow \quad & {\left[\text { Definition of } L\left(\mathfrak{A}_{\mathrm{emp}}\right)\right] } \\
& \exists q_{0} \in F \cdot \varepsilon \stackrel{\tau}{\rightarrow}_{\mathfrak{A}_{\mathrm{emp}}} q_{0} \\
\Leftrightarrow \quad & {[\text { Definition of } F] } \\
& \varepsilon \stackrel{\tau}{\rightarrow}_{\mathfrak{A}_{\mathrm{emp}}} \text { emp } \\
\Leftrightarrow \quad & {[\text { Definition of } \Delta, \operatorname{reduce}(\varphi, \varepsilon)=\tau] } \\
& \tau \models \emptyset \text { emp } \\
\Leftrightarrow \quad & {\left[\tau \in \mathrm{RSH}_{\langle\alpha\rangle}\right] } \\
& \tau \models{ }_{\Phi} \text { emp } \\
\Leftrightarrow \quad & {\left[\text { Definition of } H_{\mathrm{emp}, \Phi}^{\langle\alpha\rangle}\right] } \\
& \tau \in H_{\mathrm{emp}, \Phi}^{\langle\alpha\rangle} .
\end{aligned}
$$

The Separating Conjunction Some additional notation is needed. First, we write $\varphi \equiv \psi$ if two symbolic heaps $\varphi, \psi$ are identical up to the ordering of spatial formulas. Given symbolic heaps $\varphi, \psi$ and some variable $x$, we define

$$
[x]_{\varphi, \psi} \triangleq \begin{cases}x & \text { if } x \in \operatorname{Var}(\varphi) \cup \operatorname{Var}(\psi) \\ \varepsilon & \text { otherwise }\end{cases}
$$

Moreover, given two tuples $\mathbf{x}, \mathbf{y}$ taken from a finite set of variables $\left\{z_{1}, \ldots, z_{n}\right\}$, let $\mathbf{x} \uplus \mathbf{y} \triangleq\left[z_{1}\right]_{\varphi, \psi} \ldots . .\left[z_{n}\right]_{\varphi, \psi}$. Similar to splitting a heap $h=h_{1} \uplus h_{2}$ into two heaps, we define an operation to split symbolic heaps.

Definition 23. Let $\varphi, \psi \in \mathrm{SH}$ with free variables $\mathbf{x}_{0}^{\varphi}, \mathbf{x}_{0}^{\psi}$ and

$$
\Gamma^{\varphi}=P_{1} \mathbf{x}_{1}^{\varphi} * \ldots * P_{m} \mathbf{x}_{m}^{\varphi} \text { and } \Gamma^{\psi}=P_{1} \mathbf{x}_{1}^{\psi} * \ldots * P_{m} \mathbf{x}_{m}^{\psi}
$$

$\overline{17}$ The set of points-to assertions $U_{n}$ in this construction can even be chosen to be the empty set. 
Moreover, let $\mathbf{z}^{\varphi}, \mathbf{z}^{\psi} \subseteq\left\{z_{1}, \ldots, z_{n}\right\}$ for some $n \in \mathbb{N}$ and for each $0 \leq i \leq m$, let $\mathbf{x}_{i}^{\varphi}, \mathbf{x}_{i}^{\varphi} \subseteq \mathbf{x}_{i}$, where $\mathbf{x}_{i}$ is some finite set of variables. Then $\varphi \uplus \psi$ is defined as

$$
\begin{aligned}
\varphi \uplus \psi & \triangleq \exists\left(\mathbf{z}^{\varphi} \uplus \mathbf{z}^{\psi}\right) . \Sigma^{\varphi} * \Sigma^{\psi} * \Gamma: \Pi^{\varphi} \cup \Pi^{\psi}, \text { where } \\
\Gamma & \triangleq P_{1}\left(\mathbf{x}_{1}^{\varphi} \uplus \mathbf{x}_{1}^{\psi}\right) * \ldots * P_{m}\left(\mathbf{x}_{m}^{\varphi} \uplus \mathbf{x}_{m}^{\psi}\right)
\end{aligned}
$$

with free variables $\mathbf{x}_{0}^{\varphi} \uplus \mathbf{x}_{0}^{\psi} \cdot{ }^{18}$ Otherwise, $\varphi \uplus \psi$ is undefined.

Since inequalities between allocated variables of a symbolic heap $\varphi$ are redundant, let $\operatorname{core}(\varphi)$ denote the corresponding symbolic heap in which such inequalities are removed. Formally, $\operatorname{core}(\varphi)$ is given by:

$$
\exists \mathbf{z}^{\varphi} \cdot \Sigma^{\varphi} * \Gamma^{\varphi}: \Pi^{\varphi} \backslash\{a \neq b \mid a, b \in \operatorname{alloc}(\varphi) \cup\{\text { null }\}\} .
$$

Note that $\operatorname{core}(\varphi)$ is computable in polynomial time if $\varphi$ is reduced or each predicate is attached with variable tracking information (see Definition 10).

We are now in a position to deal with the separating conjunction of two symbolic heaps. Let $\psi_{1}, \psi_{2} \in \mathrm{SH}_{\langle\alpha\rangle}$ with $\operatorname{Var}\left(\psi_{1}\right)=\operatorname{Var}\left(\psi_{2}\right)=\mathbf{x}_{0}^{\psi_{1}}=\mathbf{x}_{0}^{\psi_{2}}$. Moreover, let $\mathfrak{A}_{\psi_{1}}, \mathfrak{A}_{\psi_{2}}$ be heap automata over $\mathrm{SH}_{\langle\alpha\rangle}$ accepting $H_{\psi_{1}, \Phi}^{\langle\alpha\rangle}$ and $H_{\psi_{2}, \Phi}^{\langle\alpha\rangle}$, respectively. We construct a heap automaton $\mathfrak{A}_{\psi_{1} * \psi_{2}}=\left(Q, \mathrm{SH}_{\langle\alpha\rangle}, \Delta, F\right)$ accepting $H_{\psi_{1} * \psi_{2}, \Phi}^{\langle\alpha\rangle}$ as follows:

$$
\begin{aligned}
& Q \triangleq 2^{\mathbf{u}} \times Q_{\mathfrak{A}_{\psi_{1}}} \times 2^{\mathbf{u}} \times Q_{\mathfrak{A}_{\psi_{2}}} \times 2^{\mathbf{u}} \\
& F \triangleq 2^{\mathbf{u}} \times F_{\mathfrak{A}_{\psi_{1}}} \times 2^{\mathbf{u}} \times F_{\mathfrak{A}_{\psi_{2}}} \times 2^{\mathbf{u}},
\end{aligned}
$$

where $\mathbf{u}$ denotes a tuple of $\alpha$ variables. The main idea of choosing this state space is that each state $(U, p, V, q, H)$ stores the current states $p, q$ of heap automata $\mathfrak{A}_{\psi_{1}}$ and $\mathfrak{A}_{\psi_{2}}$ running on two separated symbolic heaps that are obtained from splitting the originally given symbolic heap into two parts. These splitted symbolic heaps require some synchronization information encoded in sets $U, V, K$. More precisely, $U, V$ store which free variables of the original symbolic heap belong to the symbolic heap fed into $\mathfrak{A}_{\psi_{1}}$ and $\mathfrak{A}_{\psi_{2}}$, respectively. Finally, $H$ stores which variables are allocated in the symbolic heap fed into $\mathfrak{A}_{\psi_{1}}$. Correspondingly, all other allocated variables must be allocated in the symbolic heap fed into $\mathfrak{A}_{\psi_{2}}$. These sets are checked in the transition relation to ensure compositionality. Formally, for $\varphi \in \mathrm{SH}_{\langle\alpha\rangle}$ with $\left\|\Gamma^{\varphi}\right\|=m$, the transition relation $\Delta$ is given by:

$$
\begin{gathered}
\left(U_{1}, p_{1}, V_{1}, q_{1}, H_{1}\right) \ldots\left(U_{m}, p_{m}, V_{m}, q_{m}, H_{m}\right) \\
\stackrel{\varphi}{\rightarrow}_{\mathfrak{A}_{\psi_{1} * \psi_{2}}}\left(U_{0}, p_{0}, V_{0}, q_{0}, H_{0}\right) \\
\text { iff } \exists \psi_{p}, \psi_{q} \cdot \operatorname{core}(\varphi) \equiv \psi_{p} \uplus \psi_{q}
\end{gathered}
$$

$\overline{18}$ Note that technically, the predicate symbols $P_{i}$ may have different arities for each of the aforementioned symbolic heaps due to different numbers of parameters. This can easily be fixed by renaming predicate symbols. However, we chose to ignore the arity here to improve readability. 


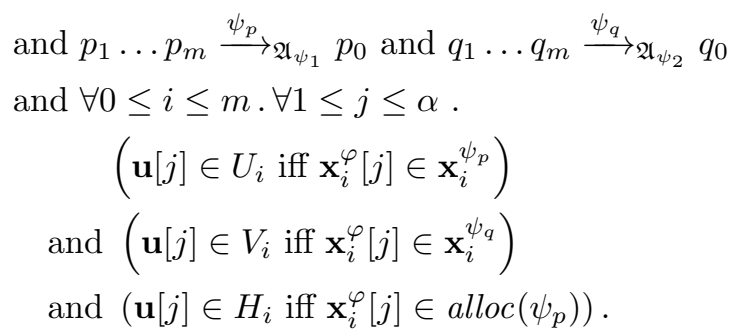

Lemma 41. $\mathfrak{A}_{\psi_{1} * \psi_{2}}$ satisfies the compositionality property.

Proof. Let $\varphi \in \mathrm{SH}_{\langle\alpha\rangle}$ with $\left\|\Gamma^{\varphi}\right\|=m$. Moreover, for each $1 \leq i \leq m$, let $\tau_{i} \in \mathrm{RSH}_{\langle\alpha\rangle}$ and $\tau \triangleq \varphi\left[P_{1}^{\varphi} / \tau_{1}, \ldots, P_{m}^{\varphi} / \tau_{m}\right]$. To improve readability, we write $U_{i}, p_{i}, V_{i}, q_{i}, H_{i}$ to denote the respective component of an automaton state $S_{i}=$ $\left(U_{i}, p_{i}, V_{i}, q_{i}, H_{i}\right) \in Q$. Let $S_{0} \in Q$. Then:

$$
\begin{aligned}
& \exists S_{1}, \ldots, S_{m} \in Q . \\
& \quad S_{1} \ldots S_{m} \stackrel{\varphi}{\rightarrow}_{\mathfrak{A}_{\psi_{1} * \psi_{2}}} S_{0} \text { and } \forall 1 \leq k \leq m \cdot \varepsilon{\stackrel{\tau_{i}}{\rightarrow} \mathfrak{A}_{\psi_{1} * \psi_{2}}} S_{k} \\
& \Leftrightarrow \quad[\text { Definition of } \Delta] \\
& \exists S_{1}, \ldots, S_{m} \in Q \\
& \exists \psi_{p}, \psi_{q} \cdot \operatorname{core}(\varphi) \equiv \psi_{p} \uplus \psi_{q} \\
& \quad \text { and } p_{1} \ldots p_{m} \stackrel{\psi_{p}}{\rightarrow} \mathfrak{A}_{\psi_{1}} p_{0} \text { and } q_{1} \ldots q_{m} \stackrel{\psi_{q}}{\rightarrow} \mathfrak{A}_{\psi_{2}} q_{0} \\
& \text { and } \forall 0 \leq i \leq m . \forall 1 \leq j \leq \alpha . \\
& \quad\left(\mathbf{u}[j] \in U_{i} \text { iff } \mathbf{x}_{i}^{\varphi}[j] \in \mathbf{x}_{i}^{\psi_{p}}\right) \\
& \quad \text { and }\left(\mathbf{u}[j] \in V_{i} \text { iff } \mathbf{x}_{i}^{\varphi}[j] \in \mathbf{x}_{i}^{\psi_{q}}\right) \\
& \quad \text { and }\left(\mathbf{u}[j] \in H_{i} \text { iff } \mathbf{x}_{i}^{\varphi}[j] \in \text { alloc }\left(\psi_{p}\right)\right) \\
& \text { and } \forall 1 \leq k \leq m . \exists \tau_{k, p}, \tau_{k, q} \cdot \\
& \text { core }\left(\tau_{k}\right) \equiv \tau_{k, p} \uplus \tau_{k, q} \\
& \text { and } \varepsilon \stackrel{\tau_{k, p}}{\longrightarrow} \mathfrak{A}_{\psi_{1}} p_{0} \text { and } \varepsilon \stackrel{\tau_{k, q}}{\longrightarrow} \mathfrak{A}_{\psi_{2}} q_{0} \\
& \text { and } \forall 1 \leq j \leq \alpha . \\
& \quad\left(\mathbf{u}[j] \in U_{k} \text { iff } \mathbf{x}_{0}^{\tau_{k}}[j] \in \mathbf{x}_{0}^{\tau_{k, p}}\right) \\
& \text { and }\left(\mathbf{u}[j] \in V_{k} \text { iff } \mathbf{x}_{0}^{\tau_{k}}[j] \in \mathbf{x}_{0}^{\tau_{k, q}}\right) \\
& \text { and }\left(\mathbf{u}[j] \in H_{k} \text { iff } \mathbf{x}_{0}^{\tau_{k}}[j] \in \text { alloc }\left(\tau_{k, p}\right)\right)
\end{aligned}
$$

To avoid introducing further notation, we do not distinguish between $\operatorname{core}(\tau)$ and $\tau$ in the following. ${ }^{19}$ Now, for each $1 \leq k \leq m$, the conditions on $U_{k}$ and $V_{k}$

19 recall that core just removes inequalities that are contained implicitly in a symbolic heap due to points-to assertions 
guarantee that the free variables of each $\tau_{k, p}$ and $\tau_{k, q}, 1 \leq k \leq m$ match with the parameters of each predicate call $P_{k}^{\psi_{p}} \mathbf{x}_{k}^{\psi_{p}}$ and $P_{k}^{\psi_{q}} \mathbf{x}_{k}^{\psi_{q}}$, respectively. Hence, the symbolic heaps

$$
\begin{gathered}
\tau_{p} \triangleq \psi_{p}\left[P_{1} / \tau_{1, p}, \ldots, P_{m} / \tau_{m, p}\right] \\
\tau_{q} \triangleq \psi_{q}\left[P_{1} / \tau_{1, q}, \ldots, P_{m} / \tau_{m, q}\right]
\end{gathered}
$$

exist. Assume, for the moment, that $\tau_{p} \uplus \tau_{q} \equiv \tau$ holds under the conditions on sets $U_{k}, V_{k}, H_{k}$. Then, the calculations from above continue as follows:

$$
\begin{aligned}
& \Leftrightarrow \text { [Compositionality of } \mathfrak{A}_{\psi_{1}}, \mathfrak{A}_{\psi_{2}} \text { ] } \\
& \exists S_{1}, \ldots, S_{m} \in Q \text {. } \\
& \exists \psi_{p}, \psi_{q} \cdot \operatorname{core}(\varphi) \equiv \psi_{p} \uplus \psi_{q} \\
& \text { and } \varepsilon{\stackrel{\tau_{p}}{\longrightarrow}}_{\mathfrak{A}_{\psi_{1}}} p_{0} \text { and } \varepsilon{\stackrel{\tau_{q}}{\longrightarrow}}_{\mathfrak{A}_{\psi_{2}}} q_{0} \\
& \text { and } \forall 0 \leq i \leq m . \forall 1 \leq j \leq \alpha \text {. } \\
& \left(\mathbf{u}[j] \in U_{i} \text { iff } \mathbf{x}_{i}^{\varphi}[j] \in \mathbf{x}_{i}^{\psi_{p}}\right) \\
& \text { and }\left(\mathbf{u}[j] \in V_{i} \text { iff } \mathbf{x}_{i}^{\varphi}[j] \in \mathbf{x}_{i}^{\psi_{q}}\right) \\
& \text { and }\left(\mathbf{u}[j] \in H_{i} \text { iff } \mathbf{x}_{i}^{\varphi}[j] \in \operatorname{alloc}\left(\psi_{p}\right)\right) \\
& \text { and } \forall 1 \leq k \leq m . \exists \tau_{k, p}, \tau_{k, q} \text {. } \\
& \operatorname{core}\left(\tau_{k}\right) \equiv \tau_{k, p} \uplus \tau_{k, q} \\
& \text { and } \forall 1 \leq j \leq \alpha \text {. } \\
& \left(\mathbf{u}[j] \in U_{k} \text { iff } \mathbf{x}_{0}^{\tau_{k}}[j] \in \mathbf{x}_{0}^{\tau_{k, p}}\right) \\
& \text { and }\left(\mathbf{u}[j] \in V_{k} \text { iff } \mathbf{x}_{0}^{\tau_{k}}[j] \in \mathbf{x}_{0}^{\tau_{k, q}}\right) \\
& \text { and }\left(\mathbf{u}[j] \in H_{k} \text { iff } \mathbf{x}_{0}^{\tau_{k}}[j] \in \operatorname{alloc}\left(\tau_{k, p}\right)\right) \\
& \Leftrightarrow \text { [Assumption: } \tau_{p} \uplus \tau_{q} \equiv \tau \text { ] } \\
& \exists \tau_{p}, \tau_{q} \cdot \operatorname{core}(\varphi) \equiv \tau_{p} \uplus \tau_{q} \\
& \text { and } \varepsilon{\stackrel{\tau_{p}}{\longrightarrow}}_{\mathfrak{A}_{\psi_{1}}} p_{0} \text { and } \varepsilon{\stackrel{\tau_{q}}{\rightarrow}}_{\mathfrak{A}_{\psi_{2}}} q_{0} \\
& \text { and } \forall 1 \leq j \leq \alpha \text {. } \\
& \left(\mathbf{u}[j] \in U_{0} \text { iff } \mathbf{x}_{0}^{\varphi}[j] \in \mathbf{x}_{0}^{\tau_{p}}\right) \\
& \text { and }\left(\mathbf{u}[j] \in V_{0} \text { iff } \mathbf{x}_{0}^{\varphi}[j] \in \mathbf{x}_{0}^{\tau_{q}}\right) \\
& \text { and }\left(\mathbf{u}[j] \in H_{0} \text { iff } \mathbf{x}_{0}^{\varphi}[j] \in \operatorname{alloc}\left(\tau_{p}\right)\right)
\end{aligned}
$$

$\Leftrightarrow[$ Definition of $\Delta]$

$$
\varepsilon \stackrel{\tau}{\rightarrow}_{\mathfrak{A}_{\psi_{1} * \psi_{2}}} S_{0} .
$$

It remains to prove the assumption $\tau_{p} \uplus \tau_{q} \equiv \tau$. Since both symbolic heaps are subformulas of $\tau$ and, by the condition on sets $H_{k}$, no variable is allocated twice, we have:

$$
\tau_{p} \uplus \tau_{q}
$$


$=\left[\right.$ Definition $\left.\tau_{p}, \tau_{q}\right]$ $\psi_{p}\left[P_{1} / \tau_{1, p}, \ldots, P_{m} / \tau_{m, p}\right] \uplus \psi_{q}\left[P_{1} / \tau_{1, q}, \ldots, P_{m} / \tau_{m, q}\right]$

$=[$ Definition of predicate replacement $]$ $\exists \mathbf{z}^{\psi_{p}} \cdot \mathbf{z}^{\tau_{1, p}} \cdot \ldots \cdot \mathbf{z}^{\tau_{m, p}}$.

$$
\begin{aligned}
& \Sigma^{\psi_{p}} * \Sigma^{\tau_{1, p}}\left[\mathbf{x}_{0}^{\tau_{1, p}} / \mathbf{x}_{1}^{\psi_{p}}\right] * \Sigma^{\tau_{m, p}}\left[\mathbf{x}_{0}^{\tau_{m, p}} / \mathbf{x}_{m}^{\psi_{p}}\right] \\
& : \Pi^{\psi_{p}} \cup \Pi^{\tau_{1, p}}\left[\mathbf{x}_{0}^{\tau_{1, p}} / \mathbf{x}_{1}^{\psi_{p}}\right] \cup \tau_{m, p}\left[\mathbf{x}_{0}^{\tau_{m, p}} / \mathbf{x}_{m}^{\psi_{p}}\right]
\end{aligned}
$$

$\uplus$

$$
\begin{aligned}
& \exists \mathbf{z}^{\psi_{q}} \cdot \mathbf{z}^{\tau_{1, q}} \cdot \ldots \cdot \mathbf{z}^{\tau_{m, q}} \cdot \\
& \quad \Sigma^{\psi_{q}} * \Sigma^{\tau_{1, q}}\left[\mathbf{x}_{0}^{\tau_{1, q}} / \mathbf{x}_{1}^{\psi_{q}}\right] * \Sigma^{\tau_{m, q}}\left[\mathbf{x}_{0}^{\tau_{m, q}} / \mathbf{x}_{m}^{\psi_{q}}\right] \\
& \quad: \Pi^{\psi_{q}} \cup \Pi^{\tau_{1, q}}\left[\mathbf{x}_{0}^{\tau_{1, q}} / \mathbf{x}_{1}^{\psi_{q}}\right] \cup \tau_{m, q}\left[\mathbf{x}_{0}^{\tau_{m, q}} / \mathbf{x}_{m}^{\psi_{q}}\right]
\end{aligned}
$$

$=[$ Definition 23]

$$
\begin{gathered}
\exists\left(\mathbf{z}^{\psi_{p}} \cdot \mathbf{z}^{\tau_{1, p}} \cdot \ldots \cdot \mathbf{z}^{\tau_{m, p}} \uplus \mathbf{z}^{\psi_{q}} \cdot \mathbf{z}^{\tau_{1, q}} \cdot \ldots \cdot \mathbf{z}^{\tau_{m, q}}\right) . \\
\quad \Sigma^{\psi_{p}} * \Sigma^{\tau_{1, p}}\left[\mathbf{x}_{0}^{\tau_{1, p}} / \mathbf{x}_{1}^{\psi_{p}}\right] * \Sigma^{\tau_{m, p}}\left[\mathbf{x}_{0}^{\tau_{m, p}} / \mathbf{x}_{m}^{\psi_{p}}\right] \\
\quad * \Sigma^{\psi_{q}} * \Sigma^{\tau_{1, q}}\left[\mathbf{x}_{0}^{\tau_{1, q}} / \mathbf{x}_{1}^{\psi_{q}}\right] * \Sigma^{\tau_{m, q}}\left[\mathbf{x}_{0}^{\tau_{m, q}} / \mathbf{x}_{m}^{\psi_{q}}\right] \\
\quad: \Pi^{\psi_{p}} \cup \Pi^{\tau_{1, p}}\left[\mathbf{x}_{0}^{\tau_{1, p}} / \mathbf{x}_{1}^{\psi_{p}}\right] \cup \tau_{m, p}\left[\mathbf{x}_{0}^{\tau_{m, p}} / \mathbf{x}_{m}^{\psi_{p}}\right] \\
\quad \cup \Pi^{\psi_{q}} \cup \Pi^{\tau_{1, q}}\left[\mathbf{x}_{0}^{\tau_{1, q}} / \mathbf{x}_{1}^{\psi_{q}}\right] \cup \tau_{m, q}\left[\mathbf{x}_{0}^{\tau_{m, q}} / \mathbf{x}_{m}^{\psi_{q}}\right]
\end{gathered}
$$

Now, by definition, we know that $\mathbf{z}^{\tau_{k, p} \uplus \tau_{k, q}}=\mathbf{z}^{\tau_{k}}$ and $\mathbf{z}^{\psi_{p} \uplus \psi_{q}}=\mathbf{z}^{\varphi}$, i.e., the existentially quantified variables reduce to $\mathbf{z}^{\varphi} \cdot \mathbf{z}^{\tau_{1}} \cdot \ldots \cdot \mathbf{z}^{\tau_{m}}$. Analogously, for each $1 \leq k \leq m$, we have

$$
\begin{aligned}
& \Sigma^{\tau_{k}}\left[\mathbf{x}_{0}^{\tau_{k}} / \mathbf{x}_{k}^{\varphi}\right] \equiv \Sigma^{\tau_{k, p}}\left[\mathbf{x}_{0}^{\tau_{k, p}} / \mathbf{x}_{k}^{\psi_{p}}\right] * \Sigma^{\tau_{k, q}}\left[\mathbf{x}_{0}^{\tau_{k, q}} / \mathbf{x}_{k}^{\psi_{q}}\right], \text { and } \\
& \Pi^{\tau_{k}}\left[\mathbf{x}_{0}^{\tau_{k}} / \mathbf{x}_{k}^{\varphi}\right]=\Pi^{\tau_{k, q}}\left[\mathbf{x}_{0}^{\tau_{k, q}} / \mathbf{x}_{k}^{\psi_{q}}\right] .
\end{aligned}
$$

Thus

$$
\begin{aligned}
& \equiv[\text { comment from above }] \\
& \exists \mathbf{z}^{\varphi} \cdot \mathbf{z}^{\tau_{1}} \cdot \ldots \cdot \mathbf{z}^{\tau_{m}} . \\
& \\
& \quad \Sigma^{\psi_{p}} * \Sigma^{\psi_{q}} \\
& \quad * \Sigma^{\tau_{1}}\left[\mathbf{x}_{0}^{\tau_{1}} / \mathbf{x}_{1}^{\varphi}\right] * \ldots * \Sigma^{\tau_{m}}\left[\mathbf{x}_{0}^{\tau_{m}} / \mathbf{x}_{m}^{\varphi}\right] \\
& \quad: \Pi^{\psi_{p}} \cup \Pi^{\psi_{q}} \\
& \quad \cup \Pi^{\tau_{1}}\left[\mathbf{x}_{0}^{\tau_{1}} / \mathbf{x}_{1}^{\varphi}\right] \cup \ldots \cup \Pi^{\tau_{m}}\left[\mathbf{x}_{0}^{\tau_{m}} / \mathbf{x}_{m}^{\varphi}\right] \\
& \equiv\left[\Sigma^{\varphi} \equiv \Sigma^{\psi_{p}} * \Sigma^{\psi_{q}}, \Pi^{\varphi}=\Pi^{\psi_{p}} \cup \Pi^{\psi_{q}}\right] \\
& \exists \mathbf{z}^{\varphi} \cdot \mathbf{z}^{\tau_{1}} \cdot \ldots \cdot \mathbf{z}^{\tau_{m}} \cdot \\
& \quad \Sigma^{\varphi} * \Sigma^{\tau_{1}}\left[\mathbf{x}_{0}^{\tau_{1}} / \mathbf{x}_{1}^{\varphi}\right] * \ldots * \Sigma^{\tau_{m}}\left[\mathbf{x}_{0}^{\tau_{m}} / \mathbf{x}_{m}^{\varphi}\right] \\
& \quad: \Pi^{\varphi} \cup \Pi^{\tau_{1}}\left[\mathbf{x}_{0}^{\tau_{1}} / \mathbf{x}_{1}^{\varphi}\right] \cup \ldots \cup \Pi^{\tau_{m}}\left[\mathbf{x}_{0}^{\tau_{m}} / \mathbf{x}_{m}^{\varphi}\right]
\end{aligned}
$$

$\equiv$ [Definition of predicate replacement $]$ 


$$
\begin{aligned}
& \varphi\left[P_{1}^{\varphi} \mathbf{x}_{1}^{\varphi} / \tau_{1}, \ldots P_{m}^{\varphi} \mathbf{x}_{m}^{\varphi} / \tau_{m}\right] \\
\equiv & {[\text { Definition of } \tau] } \\
& \tau .
\end{aligned}
$$

Hence, $\mathfrak{A}_{\psi_{1} * \psi_{2}}$ satisfies the compositionality property.

Lemma 42. $L\left(\mathfrak{A}_{\psi_{1} * \psi_{2}}\right)=H_{\psi_{1} * \psi_{2}, \Phi}^{\langle\alpha\rangle}$.

Proof. Let $\tau \in \mathrm{RSH}_{\langle\alpha\rangle}$. Then

$$
\begin{aligned}
& \tau \in L\left(\mathfrak{A}_{\psi_{1} * \psi_{2}}\right) \\
& \left.\Leftrightarrow \quad \text { [Definition of } L\left(\mathfrak{A}_{\psi_{1} * \psi_{2}}\right)\right] \\
& \exists S_{0}=\left(U_{0}, p_{0}, V_{0}, q_{0}, H_{0}\right) \in F . \varepsilon \stackrel{\tau}{\rightarrow}_{\mathfrak{A}_{\psi_{1} * \psi_{2}}} S_{0} \\
& \Leftrightarrow \quad \text { Definition of } \Delta] \\
& \exists S_{0}=\left(U_{0}, p_{0}, V_{0}, q_{0}, H_{0}\right) \in F . \\
& \exists \psi_{p}, \psi_{q} \cdot \operatorname{core}(\tau) \equiv \psi_{p} \uplus \psi_{q} \\
& \text { and } \varepsilon{\stackrel{\psi_{p}}{\longrightarrow}}_{\mathfrak{A}_{\psi_{1}}} p_{0} \text { and } \varepsilon \stackrel{\psi_{q}}{\longrightarrow} \mathfrak{A}_{\psi_{2}} q_{0} \\
& \text { and } \forall 1 \leq j \leq \alpha \text {. } \\
& \left(\mathbf{u}[j] \in U_{0} \text { iff } \mathbf{x}_{0}^{\tau}[j] \in \mathbf{x}_{0}^{\psi_{p}}\right) \\
& \text { and }\left(\mathbf{u}[j] \in V_{0} \text { iff } \mathbf{x}_{0}^{\tau}[j] \in \mathbf{x}_{0}^{\psi_{q}}\right) \\
& \text { and }\left(\mathbf{u}[j] \in H_{0} \text { iff } \mathbf{x}_{0}^{\tau}[j] \in \operatorname{alloc}\left(\psi_{p}\right)\right) \\
& \Leftrightarrow\left[p_{0} \in F_{\mathfrak{A}_{\psi_{1}}}, q_{0} \in F_{\mathfrak{A}_{\psi_{2}}} \text {, Definition of } L\left(\mathfrak{A}_{\psi_{1}}\right), L\left(\mathfrak{A}_{\psi_{2}}\right)\right] \\
& \exists \psi_{p}, \psi_{q} \cdot \operatorname{core}(\tau) \equiv \psi_{p} \uplus \psi_{q} \\
& \text { and } \psi_{p} \models_{\Phi} \psi_{1} \text { and } \psi_{q} \models_{\Phi} \psi_{2} \\
& \Leftrightarrow\left[\operatorname{core}(\tau) \models_{\emptyset} \tau \text { and } \tau \models_{\emptyset} \operatorname{core}(\tau)\right] \\
& \exists \psi_{p}, \psi_{q} \cdot \psi_{p} \uplus \psi_{q} \models_{\emptyset} \tau \text { and } \tau \models_{\emptyset} \psi_{p} \uplus \psi_{q} \\
& \text { and } \psi_{p} \models_{\Phi} \psi_{1} \text { and } \psi_{q} \models_{\Phi} \psi_{2} \text {. }
\end{aligned}
$$

To complete the proof we show

$$
\begin{aligned}
& \quad \exists \psi_{p}, \psi_{q} \cdot \psi_{p} \uplus \psi_{q} \models_{\emptyset} \tau \text { and } \tau \models_{\emptyset} \psi_{p} \uplus \psi_{q} \\
& \quad \text { and } \psi_{p} \models_{\Phi} \psi_{1} \text { and } \psi_{q} \models_{\Phi} \psi_{2} \\
& \text { iff } \tau \models_{\Phi} \psi_{1} * \psi_{2} \text {. }
\end{aligned}
$$

This, together with the previously shown equivalences, immediately yields

$$
\tau \in L\left(\mathfrak{A}_{\psi_{1} * \psi_{2}}\right) \text { iff } \tau \models_{\Phi} \psi_{1} * \psi_{2} \text { iff } \tau \in H_{\psi_{1} * \psi_{2}}^{\Phi} .
$$

For the first direction, we have

$$
s, h \models_{\emptyset} \tau
$$




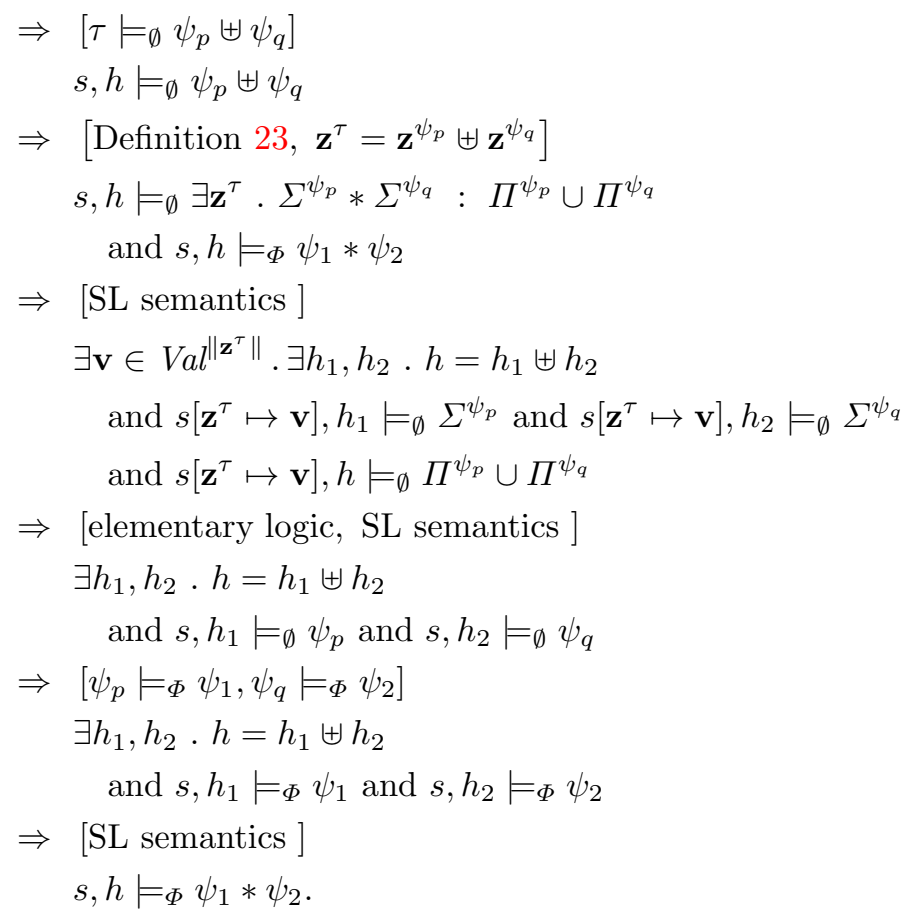

Hence, $\tau \models_{\Phi} \psi_{1} * \psi_{2}$. For the converse direction, we have

$\tau \models_{\Phi} \psi_{1} * \psi_{2}$

$\Rightarrow \quad$ Definition entailment $]$

$\forall(s, h) . s, h \models_{\Phi} \tau$ implies $s, h \models_{\Phi} \psi_{1} * \psi_{2}$

$\Rightarrow[\tau$ reduced and well-determined $]$

$\exists(s, h) . s, h \models_{\emptyset} \tau$ and $s, h \models_{\Phi} \psi_{1} * \psi_{2}$

$\Rightarrow[\tau=\exists \mathbf{z} \cdot \Sigma: \Pi$, SL semantics $]$

$\exists(s, h) . \exists h_{1}, h_{2} \cdot h=h_{1} \uplus h_{2}$

and $\exists \mathbf{z} \in \operatorname{Val}^{\|\mathbf{z}\|} . s[\mathbf{z} \mapsto \mathbf{v}], h \models_{\emptyset} \Sigma$

and $s[\mathbf{z} \mapsto \mathbf{v}], h \models_{\emptyset} \Pi$

and $s, h_{1} \models_{\Phi} \psi_{1}$ and $s, h_{2} \models_{\Phi} \psi_{2}$

$\Rightarrow \quad[$ SL semantics $]$

$$
\begin{aligned}
& \exists(s, h) . \exists h_{1}, h_{2} \cdot h=h_{1} \uplus h_{2} \text { and } \exists \mathbf{z} \in \operatorname{Val}^{\|\mathbf{z}\|} . \\
& h=\{s[\mathbf{z} \mapsto \mathbf{v}](x) \mapsto s[\mathbf{z} \mapsto \mathbf{v}](\mathbf{y}) \mid x \mapsto(\mathbf{y}) \text { in } \Sigma\} \\
& \quad \text { and } s[\mathbf{z} \mapsto \mathbf{v}], h \models_{\emptyset} \Pi \\
& \text { and } s, h_{1} \models_{\Phi} \psi_{1} \text { and } s, h_{2} \models_{\Phi} \psi_{2} .
\end{aligned}
$$

Now, we choose $\psi_{p}, \psi_{q}$ such that $\operatorname{core}(\tau)=\psi_{p} \uplus \psi_{q}$ by adding exactly those points-to assertions $x \mapsto(\mathbf{y})$ to $\Sigma^{\psi_{p}}$, where $s[\mathbf{z} \mapsto \mathbf{v}](x) \in \operatorname{dom}\left(h_{1}\right)$. All other 
points-to assertions are added to $\psi_{q}$. The existentially quantified variables and pure formulas are chosen such that both symbolic heaps are well-determined. This is always possible, because $\psi_{1}$ and $\psi_{2}$ are well-determined on their own. Then $s, h_{1} \models_{\emptyset} \psi_{p}$ and $s, h_{2} \models_{\emptyset} \psi_{q}$. Since both $\psi_{p}$ and $\psi_{q}$ are well-determined, this implies $\psi_{p} \models_{\Phi} \psi_{1}$ and $\psi_{q} \models_{\Phi} \psi_{q}$. Moreover, since each points-to assertion and each pure formula in the core occurs in $\psi_{p}$ or $\psi_{q}$, we have $\operatorname{core}(\tau)=\psi_{p} \uplus \psi_{q}$. Hence, $\tau \models_{\Phi} \psi_{1} * \psi_{2}$ implies

$$
\begin{aligned}
& \exists \psi_{p}, \psi_{q} \cdot \psi_{p} \uplus \psi_{q} \models_{\emptyset} \tau \text { and } \tau \models_{\emptyset} \psi_{p} \uplus \psi_{q} \\
& \text { and } \psi_{p} \models_{\Phi} \psi_{1} \text { and } \psi_{q} \models_{\Phi} \psi_{2} .
\end{aligned}
$$

The Pure Formula Dealing with pure formulas is rather straightforward, because we already know how to track equalities and inequalities using the construction from Definition 10.

Let $\vartheta \in \mathrm{SH}_{\langle\alpha\rangle}$ such that $H_{\vartheta, \Phi}^{\langle\alpha\rangle}$ there exists a heap automaton, say $\mathfrak{A}_{\vartheta}$, accepting $H_{\vartheta, \Phi}^{\langle\alpha\rangle}$. Moreover, let $\Pi$ be some finite set of pure formulas over the set of free variables of $\vartheta$. We construct a heap automaton $\mathfrak{A}_{\vartheta}: \Pi=\left(Q, \mathrm{SH}_{\langle\alpha\rangle}, \Delta, F\right)$ accepting $H_{\psi, \Phi}^{\langle\alpha\rangle}$, where $\psi \triangleq \exists \mathbf{z}^{\vartheta} . \Sigma^{\vartheta} * \Gamma^{\vartheta}: \Pi^{\vartheta} \cup \Pi$, as follows:

$$
Q \triangleq Q_{\mathfrak{A}_{\vartheta}} \times\{0,1\} \quad F \triangleq F_{\mathfrak{A}_{\vartheta}} \times\{1\}
$$

Moreover, for $\varphi \in \mathrm{SH}_{\langle\alpha\rangle}$ with $\left\|\Gamma^{\varphi}\right\|=m$, the transition relation $\Delta$ is given by:

$$
\begin{aligned}
& \quad\left(p_{1}, q_{1}\right) \ldots\left(p_{m}, q_{m}\right) \stackrel{\varphi}{\rightarrow}_{\mathfrak{A}_{\vartheta}: \Pi}\left(p_{0}, q_{0}\right) \\
& \text { iff } p_{1} \ldots p_{m} \stackrel{\varphi}{\rightarrow}_{\mathfrak{A}_{\vartheta}} p_{0} \text { and } \bigwedge_{0 \leq i \leq m} q_{i}=1 \text { iff } \Pi \subseteq \Pi_{i},
\end{aligned}
$$

where $\Pi_{i}$ is the set of pure formulas obtained from the tracking automaton (see Definition 10) that is available by Remark 4.

Lemma 43. $\mathfrak{A}_{\vartheta}: \Pi$ satisfies the compositionality property.

Proof. Let $\varphi \in \mathrm{SH}_{\langle\alpha\rangle}$ with $\left\|\Gamma^{\varphi}\right\|=m$. Moreover, for each $1 \leq i \leq m$, let $\tau_{i} \in \mathrm{RSH}_{\langle\alpha\rangle}$ and $\tau \triangleq \varphi\left[P_{1}^{\varphi} / \tau_{1}, \ldots, P_{m}^{\varphi} / \tau_{m}\right]$. For each $1 \leq i \leq m$, let $\Pi_{i}$ be the set of pure formulas obtained by the tracking automaton (cf. Definition 10) for $\tau_{i}$. Note that this information is available without running the full automaton again due to Remark 4 . Then, for each $\left(p_{0}, q_{0}\right) \in Q$, we have

$$
\begin{aligned}
& \varepsilon \stackrel{\tau}{\rightarrow}_{\mathfrak{A}_{\vartheta}: \Pi}\left(p_{0}, q_{0}\right) \\
\Leftrightarrow & {[\text { Definition of } \Delta] } \\
& \varepsilon \stackrel{\tau}{\rightarrow}_{\mathfrak{A}} p_{0} \text { and } q_{0}=1 \text { iff } \Pi \subseteq \Pi_{0} \\
& {\left[\text { for each } 1 \leq i \leq m \text { set } q_{i}=1 \text { iff } \Pi \subseteq \Pi_{i}\right] }
\end{aligned}
$$




$$
\begin{aligned}
& \exists q_{1}, \ldots, q_{m} \in\{0,1\} \text {. } \\
& \mathcal{\varepsilon}^{\rightarrow} \stackrel{\tau}{\rightarrow}_{\mathfrak{A}} p_{0} \text { and } \bigwedge_{0 \leq i \leq m} q_{i}=1 \text { iff } \Pi \subseteq \Pi_{i} \\
& \Leftrightarrow \quad \text { [Compositionality of } \mathfrak{A}_{\vartheta} \text { ] } \\
& \exists q_{1}, \ldots, q_{m} \in\{0,1\} . \exists p_{1}, \ldots, p_{m} \in Q_{\mathfrak{A}_{\vartheta}} . \\
& p_{1} \ldots p_{m} \stackrel{\varphi}{\rightarrow}_{\mathfrak{A}_{\vartheta}} p_{0} \text { and } \bigwedge_{0 \leq i \leq m} q_{i}=1 \text { iff } \Pi \subseteq \Pi_{i} \\
& \text { and } \forall 1 \leq i \leq m \cdot \varepsilon \stackrel{\tau_{i}}{\longrightarrow} \mathfrak{A}_{\vartheta} p_{i} \\
& \Leftrightarrow \text { [Definition of } Q \text {, regrouping] } \\
& \exists\left(p_{1}, q_{1}\right), \ldots,\left(p_{m}, q_{m}\right) \in Q . \\
& p_{1} \ldots p_{m} \stackrel{\varphi}{\rightarrow}_{\mathfrak{A}_{\vartheta}} p_{0} \text { and } \bigwedge_{0 \leq i \leq m} q_{i}=1 \text { iff } \Pi \subseteq \Pi_{i} \\
& \text { and } \forall 1 \leq i \leq m \cdot \varepsilon \stackrel{\tau_{i}}{\rightarrow} \mathfrak{A}_{\vartheta} p_{i} \text { and } q_{i}=1 \text { iff } \Pi \subseteq \Pi_{i} \\
& \Leftrightarrow[\text { Definition of } \Delta] \\
& \exists\left(p_{1}, q_{1}\right), \ldots,\left(p_{m}, q_{m}\right) \in Q \text {. } \\
& \left(p_{1}, q_{1}\right) \ldots\left(p_{m}, q_{m}\right) \stackrel{\varphi}{\rightarrow}_{\mathfrak{A}_{\vartheta}: \Pi}\left(p_{0}, q_{0}\right) \\
& \text { and } \forall 1 \leq i \leq m \cdot \varepsilon{\stackrel{\tau_{i}}{\rightarrow}}_{\mathfrak{A}_{\vartheta}: \Pi}\left(p_{i}, q_{i}\right) \text {. }
\end{aligned}
$$

Lemma 44. $L\left(\mathfrak{A}_{\vartheta}: \Pi\right)=H_{\psi, \Phi}^{\langle\alpha\rangle}$, where $\psi \triangleq \exists \mathbf{z}^{\vartheta} \cdot \Sigma^{\vartheta} * \Gamma^{\vartheta}: \Pi \vartheta \cup \Pi$ and $\vartheta \in$ $\mathrm{SH}_{\langle\alpha\rangle}^{\Phi}$.

Proof. Let $\tau \in \mathrm{RSH}_{\langle\alpha\rangle}$. Then

$$
\begin{aligned}
& \tau \in L\left(\mathfrak{A}_{\vartheta}: \Pi\right) \\
\Leftrightarrow & {\left[\text { Definition of } L\left(\mathfrak{A}_{\vartheta}: \Pi\right)\right] } \\
& \exists\left(p_{0}, q_{0}\right) \in F \cdot \varepsilon \stackrel{\tau}{\rightarrow}_{\mathfrak{A}_{\vartheta}: \Pi}\left(p_{0}, q_{0}\right) \\
\Leftrightarrow & {[\text { Definition of } F] } \\
& \exists p_{0} \in F_{\mathfrak{A}_{\vartheta}} \cdot \varepsilon \stackrel{\tau}{\rightarrow}_{\mathfrak{A}_{\vartheta}: \Pi}\left(p_{0}, 1\right) \\
\Leftrightarrow & {\left[\text { Definition of } \Delta, q_{0}=1\right] } \\
& \exists p_{0} \in F_{\mathfrak{A}_{\vartheta}} \cdot \varepsilon \stackrel{\tau}{\rightarrow} \mathfrak{A}_{\vartheta} p_{0} \\
& \quad \text { and } \quad \bigwedge_{0 \leq i \leq\left\|\Gamma^{\tau}\right\|} q_{i}=1 \text { iff } \Pi \subseteq \Pi_{i} \\
\Leftrightarrow & {\left[\left\|\Gamma^{\tau}\right\|=0, q_{0}=1\right] } \\
& \exists p_{0} \in F_{\mathfrak{A}_{\vartheta}} \cdot \varepsilon \stackrel{\tau}{\rightarrow} \mathfrak{A}_{\vartheta} p_{0} \text { and } \Pi \subseteq \Pi_{0} \\
\Leftrightarrow & {\left[\text { Definition of } L\left(\mathfrak{A}_{\vartheta}\right)\right] } \\
& \tau \in L\left(\mathfrak{A}_{\vartheta}\right) \text { and } \Pi \subseteq \Pi_{0}
\end{aligned}
$$




$$
\begin{aligned}
\Leftrightarrow & {\left[L\left(\mathfrak{A}_{\vartheta}\right)=H_{\vartheta, \Phi}^{\langle\alpha\rangle}\right] } \\
& \tau \in H_{\vartheta, \Phi}^{\langle\alpha\rangle} \text { and } \Pi \subseteq \Pi_{0} \\
\Leftrightarrow & {\left.\left[\text { Definition of } \Pi_{0} \text { (see Definition } 8\right)\right] } \\
& \tau \in H_{\vartheta, \Phi}^{\langle\alpha\rangle} \text { and } \Pi \subseteq\left\{x \sim y \mid x \sim_{\tau} y\right\} \\
\Leftrightarrow & {\left[\text { Definition } H_{\vartheta, \Phi}^{\langle\alpha\rangle}, \sim_{\tau}\right] } \\
& \tau \models_{\Phi} \vartheta \text { and } \tau \models_{\emptyset} \Pi \\
\Leftrightarrow & {\left[\psi \triangleq \exists \mathbf{z}^{\vartheta} . \Sigma^{\vartheta} * \Gamma^{\vartheta}: \Pi^{\vartheta} \cup \Pi\right] } \\
& \quad \tau \models{ }_{\Phi} \psi \\
\Leftrightarrow & {\left[\text { Definition } H_{\psi, \Phi}^{\langle\alpha\rangle}\right] } \\
& \tau \in H_{\psi, \Phi}^{\langle\alpha\rangle} .
\end{aligned}
$$

The Existential Quantification In order to deal with existential quantifiers, we exploit the following structural property.

Lemma 45. For $\Phi \in \mathrm{SID}$, let $\tau \in \mathrm{RSH}_{\alpha}$ be a well-determined symbolic heap and $\psi \in \mathrm{SH}_{\alpha}^{\Phi}$. Moreover, let $x \notin \operatorname{Var}(\tau)$ and $\mathbf{x}_{0}^{\psi}=\mathbf{x}_{0}^{\tau} \cdot x$. Then $\tau \models_{\Phi} \exists x$. $\psi$ holds if and only if there exists $y \in \operatorname{Var}(\tau)$ such that $\exists \mathbf{z}^{\tau} . \Sigma^{\tau}: \Pi^{\tau} \cup\{x=y\} \models_{\Phi} \psi$.

Proof. To improve readability, we write $\tau_{y}$ as a shortcut for $\exists \mathbf{z}^{\tau} . \Sigma^{\tau}: \Pi^{\tau} \cup\{x=$ $y\}$.

"only-if" Towards a contradiction, assume that $\tau \models_{\Phi} \exists x . \psi$ holds, but for each $y \in \operatorname{Var}(\tau)$, we have $\tau_{y} \forall_{\Phi} \psi$. Since $\tau$ is established and well-determined and $x \notin \operatorname{Var}(\tau)$, we know that $\tau_{y}$ is established and well-determined as well. Thus, let $(s, h) \in \operatorname{Models}\left(\tau_{y}\right)$ be the unique model of $\tau_{y}$ up to isomorphism. Clearly, $s(x)=s(y)$ due to the pure formula $x=y$. Then, by assumption, $s, h \not \models_{\Phi} \psi$.

Now, let $\left(s^{\prime}, h^{\prime}\right) \in \operatorname{Models}(\tau)$ be the unique model of $\tau$ up to isomorphism. Since $\tau \models_{\Phi} \exists x . \psi$, applying the SL semantics yields that there exists $v \in$ Val such that $s^{\prime}[x \mapsto v], h^{\prime} \models_{\Phi} \psi$. Moreover, $v$ is drawn from the image (or codomain) of $s^{\prime}$ and $\operatorname{dom}\left(h^{\prime}\right)$, because $\psi$ is established. By Lemma 10 and $x \notin \operatorname{Var}(\tau)$, we know that $s^{\prime}[x \mapsto v], h^{\prime} \models_{\emptyset} \tau$. Observe that for each of these values $v$ there exists at least one variable $y \in \operatorname{Var}(\tau)$ such that $y$ is evaluated to $v{ }^{20}$ Then $s^{\prime}[x \mapsto$ $v], h^{\prime} \models_{\emptyset} \tau_{y}$ holds for some $y \in \operatorname{Var}(\tau)$ that is evaluated to $v$. However, since

${ }^{20}$ For $v \in \operatorname{codom}\left(s^{\prime}\right)$ this is clear, because there exists $y \in \operatorname{dom}\left(s^{\prime}\right)$ with $s^{\prime}(y)=v$. Otherwise, if $v \in \operatorname{dom}\left(h^{\prime}\right)$, applying the SL semantics yields that there exists $\mathbf{u} \in$ $\operatorname{codom}\left(s^{\prime}\right) \cup \operatorname{dom}\left(h^{\prime}\right)$ such that $s^{\prime}\left[\mathbf{z}^{\tau} \mapsto \mathbf{u}\right], h^{\prime} \models_{\emptyset} \Sigma^{\tau}: \Pi^{\tau}$. Then, since $\operatorname{dom}\left(h^{\prime}\right)=$ $\left\{s^{\prime}\left[\mathbf{z}^{\tau} \mapsto \mathbf{u}\right](z) \mid z \mapsto(-)\right.$ occurs in $\left.\Sigma^{\tau}\right\}, v$ is contained in $\operatorname{codom}\left(s^{\prime}\left[\mathbf{z}^{\tau} \mapsto \mathbf{u}\right]\right)$. Thus there exists some variable $y \in \operatorname{dom}\left(s^{\prime}\left[\mathbf{z}^{\tau} \mapsto \mathbf{u}\right]\right)=\operatorname{Var}(\tau)$ with $s^{\prime}\left[\mathbf{z}^{\tau} \mapsto \mathbf{u}\right](y)=v$. 
$s^{\prime}[x \mapsto v], h^{\prime} \models_{\Phi} \psi$, this means that $\tau_{y} \models_{\Phi} \psi$. This contradicts our assumption $\tau_{y} \not \forall_{\Phi} \psi$ for each $y \in \operatorname{Var}(\tau){ }^{21}$

"if" Assume there exists $y \in \operatorname{Var}(\tau)$ such that $\tau_{y} \models_{\Phi} \psi$. Thus, for each $s, h \models_{\emptyset} \tau_{y}$, we have $s, h \models_{\Phi} \psi$. Furthermore, since $\tau$ is established and $x$ occurs in $\tau_{y}$ in the pure formula $x=y$ only, we know that

$$
s(x) \in s\left(\mathbf{x}_{0}^{\tau}\right) \cup \operatorname{dom}(h) .
$$

In particular, $s, h \models_{\emptyset} \tau$ holds, because only one pure formula is added by $\tau_{y}$. Moreover, since $x \notin \operatorname{Var}(\tau),\left.s\right|_{\mathbf{x}_{0}^{\tau}}, h \models_{\emptyset} \tau$ holds by Lemma 10. Then

$$
\begin{aligned}
& s, h \models_{\emptyset} \tau_{y} \\
& \left.\Rightarrow \quad \text { Assumption: } \tau_{y} \models_{\Phi} \psi\right] \\
& s, h \models_{\emptyset} \psi \\
& \Rightarrow \quad \text { applying }(\dagger), v=s(x)] \\
& \exists v \in s\left(\mathbf{x}_{0}^{\tau}\right) \cup \operatorname{dom}(h) .\left(s\left\lceil_{\mathbf{x}_{0}^{\tau}}\right)[x \mapsto v]=s \text { and } s, h \models_{\emptyset} \psi\right. \\
& \Rightarrow \quad\left[\left(s\left\lceil\mathbf{x}_{0}^{\tau}\right)[x \mapsto v]=s\right]\right. \\
& \exists v \in s\left(\mathbf{x}_{0}^{\tau}\right) \cup \operatorname{dom}(h) .\left(s\left\lceil_{\mathbf{x}_{0}^{\tau}}\right)[x \mapsto v], h \models_{\emptyset} \psi\right. \\
& \Rightarrow\left[s\left(\mathbf{x}_{0}^{\tau}\right) \cup \operatorname{dom}(h) \subseteq V a l\right] \\
& \exists v \in \operatorname{Val} .\left(s \Upsilon_{\mathbf{x}_{0}^{\tau}}\right)[x \mapsto v], h \models_{\emptyset} \psi \\
& \Rightarrow \text { [SL semantics }] \\
& \left(\left.s\right|_{\mathbf{x}_{0}^{\tau}}\right), h \models_{\emptyset} \exists x . \psi .
\end{aligned}
$$

Hence, each model of $\tau$ is also a model of $\exists x$. $\psi$, i.e., $\tau \models_{\Phi} \exists x . \psi$.

Note that the proof of the lemma from above works analogously if the additional free variable $x$ occurs at a different position in the tuple of free variables.

Following the previous lemma, the main idea is to nondeterministically guess some variable $y$ of an unfolding $\tau$ and verify that $\tau:\{x=y\}$ belongs to $H_{\psi, \Phi}^{\langle\alpha\rangle}$. Since this construction increases the number of free variables, we remark that $y$ has to be chosen carefully such that a symbolic heap in $\mathrm{SH}_{\alpha}$ is obtained. In particular, if $y$ does not belong to the root node of an unfolding tree, the corresponding predicate must either have an arity smaller than $\alpha$, or $y$ is equal to some other free variable. However, each $y$ occurring in the root node of an unfolding tree may be chosen.

Before we present a formal construction, we first define how a symbolic heap $\varphi$ is modified in the following.

Definition 24. Let $\mathbf{x}$ be a tuple of variables with $\|\mathbf{x}\|=n$. Moreover, let $1 \leq$ $k \leq n+1$. Then, the tuple of variables in which a fresh variable $x$ is placed at

${ }^{21}$ Note that $\tau_{y}$ is well-determined. Thus, one common model between $\psi$ and $\tau_{y}$ is sufficient to prove the entailment $\tau_{y} \models_{\Phi} \psi$. 
position $k$ is given by:

$$
\mathbf{x}[x \downarrow k] \triangleq \mathbf{x}[1] \ldots \mathbf{x}[k-1] x \mathbf{x}[k+1] \ldots \mathbf{x}[n] .
$$

Now, let $\varphi$ be a symbolic heap with $\left\|\mathbf{x}_{0}^{\varphi}\right\|=\beta<\alpha$. Then, the symbolic heap $\varphi[x \downarrow k]$ is defined as $\varphi$ except for the tuple of free variables being $\mathbf{x}_{0}^{\varphi}[x \downarrow k]$. Furthermore, for some $1 \leq i \leq\left\|\Gamma^{\varphi}\right\|$ and $1 \leq \ell \leq\left\|\mathbf{x}_{i}^{\varphi}\right\|+1$, the symbolic heap $\varphi[x \downarrow i, \ell]$ is defined as $\varphi$ except for the tuple of parameters of the $i$-th predicate call being $\mathbf{x}_{i}^{\varphi[x \downarrow i, \ell]}=\mathbf{x}_{i}^{\varphi}[x \downarrow \ell]$. Otherwise, $\varphi[x \downarrow k]$ as well as $\varphi[x \downarrow i, k]$ are undefined.

Now, let $\mathfrak{A}_{\psi}$ be a heap automaton accepting $H_{\psi, \Phi}^{\langle\alpha\rangle}$ and $\mathfrak{A}_{\text {TRACK }}$ be the tracking automaton introduced in Definition 10. We construct a heap automaton $\mathfrak{A}_{\exists z . \psi}=$ $\left(Q, \mathrm{SH}_{\langle\alpha\rangle}, \Delta, F\right)$ accepting $H_{\exists x . \psi, \Phi}^{\langle\alpha\rangle}$, where $x$ is the $k$-th free variable of $\psi$, as follows:

$$
Q \triangleq Q_{\mathfrak{A}_{\psi}} \times\{0,1, \ldots, \alpha\} \times Q_{\mathfrak{A}_{\mathrm{TRACK}}} \quad F \triangleq F_{\mathfrak{A}_{\psi}} \times\{k\} \times Q_{\mathfrak{A}_{\mathrm{TRACK}}}
$$

Moreover, for $\varphi \in \mathrm{SH}_{\langle\alpha\rangle}$ with $\left\|\Gamma^{\varphi}\right\|=m$, the transition relation $\Delta$ is given by:

$$
\begin{aligned}
& \left(p_{1}, q_{1}, r_{m}\right) \ldots\left(p_{m}, q_{m}, r_{m}\right) \stackrel{\varphi}{\rightarrow}_{\mathfrak{A}_{\exists z . \psi}}\left(p_{0}, q_{0}, r_{0}\right) \\
\text { iff } \quad & p_{1} \ldots p_{m} \stackrel{\vartheta}{\rightarrow}_{\mathfrak{A}_{\psi}} p_{0} \text { and } r_{1} \ldots r_{m} \stackrel{\vartheta}{\rightarrow}_{\mathfrak{A}_{\mathrm{TRACK}}} r_{0},
\end{aligned}
$$

where $\vartheta$ adheres to one of the following cases:

1. $q_{0}=q_{1}=\ldots=q_{m}=0$ and $\vartheta=\varphi$, or

2. $q_{0}=\ell>0$ and there exists exactly one $1 \leq j \leq m$ such that $q_{j}=\ell^{\prime}>0$ and $\vartheta=(\varphi[x \downarrow \ell])\left[x \downarrow j, \ell^{\prime}\right]$, or

3. $q_{0}=\ell>0$ and $\sum_{1 \leq i \leq m} q_{i}=0$ and $\vartheta=(\varphi[x \downarrow \ell]):\{x=y\}$ for some $y \in \operatorname{Var}(\varphi)$.

Here, $\varphi:\{y=x\}$ is a shortcut for the symbolic heap $\exists \mathbf{z}^{\varphi} \cdot \Sigma^{\varphi} * \Gamma^{\varphi}: \Pi^{\varphi} \cup\{x=$ $y\}$. Moreover, the annotations of each predicate call $P_{i}^{\vartheta} \mathbf{x}_{i}^{\vartheta}$ are set to the sets contained in $r_{i}=\left(A_{i}, \Pi_{i}\right)$ instead of using existing annotations. We remark that this construction is highly non-deterministic. Furthermore, note that the automata rejects a symbolic heap if some $\vartheta$ does not belong to $\mathrm{SH}_{\langle\alpha\rangle}$, because it is not a top-level formula and has more than $\alpha$ free variables.

Lemma 46. $\mathfrak{A}_{\exists z . \psi}$ satisfies the compositionality property.

Proof. Let $\varphi \in \mathrm{SH}_{\langle\alpha\rangle}$ with $\left\|\Gamma^{\varphi}\right\|=m$. Moreover, for each $1 \leq i \leq m$, let $\tau_{i} \in \mathrm{RSH}_{\langle\alpha\rangle}$ and $\tau \triangleq \varphi\left[P_{1}^{\varphi} / \tau_{1}, \ldots, P_{m}^{\varphi} / \tau_{m}\right]$. Assume $\varepsilon \stackrel{\tau}{\rightarrow}_{\mathfrak{A}_{\text {TRACK }}}\left(p_{0}, q_{0}, r_{0}\right)$. By definition of $\Delta$, we either have $q_{0}=0$ or $q_{0}>0$. We proceed by case distinction.

The case $q_{0}=0$

$$
\varepsilon \stackrel{\tau}{\rightarrow}_{\mathfrak{A}_{\text {TRACK }}}\left(p_{0}, 0, r_{0}\right)
$$

$\Leftrightarrow \quad[$ Definition of $\Delta$ (only first case applicable) $]$ 


$$
\begin{aligned}
& \varepsilon \stackrel{\tau}{\rightarrow}_{\mathfrak{A}_{\psi}} p_{0} \text { and } \varepsilon \stackrel{\tau}{\rightarrow} \mathfrak{A}_{\mathrm{TRACK}} r_{0} \\
& \left.\Leftrightarrow \quad \text { Compositionality of } \mathfrak{A}_{\psi}, \mathfrak{A}_{\mathrm{TRACK}}\right] \\
& \exists p_{1}, \ldots, p_{m} \in Q_{\mathfrak{A}_{\psi}} . \exists r_{1}, \ldots, r_{m} \in Q_{\mathfrak{A}_{\mathrm{TRACK}}} . \\
& p_{1} \ldots p_{m} \stackrel{\varphi}{\rightarrow}_{\mathfrak{A}_{\psi}} p_{0} \text { and } \forall 1 \leq i \leq m \cdot \varepsilon{\stackrel{\tau_{i}}{\rightarrow}}_{\mathfrak{A}_{\psi}} p_{i} \\
& \text { and } r_{1} \ldots r_{m} \stackrel{\varphi}{\rightarrow}_{\mathfrak{A}_{\mathrm{TRACK}}} r_{0} \text { and } \forall 1 \leq i \leq m \cdot \varepsilon{\stackrel{\tau_{i}}{\rightarrow}}_{\mathfrak{A}_{\mathrm{TRACK}}} r_{i} \\
& \Leftrightarrow \quad\left[\text { choose } q_{1}=\ldots=q_{m}=0\right] \\
& \exists p_{1}, \ldots, p_{m} \in Q_{\mathfrak{A}_{\psi}} . \exists r_{1}, \ldots, r_{m} \in Q_{\mathfrak{A}_{\mathrm{TRACK}}} . \\
& p_{1} \ldots p_{m} \stackrel{\varphi}{\rightarrow}_{\mathfrak{A}_{\psi}} p_{0} \text { and } \forall 1 \leq i \leq m \cdot \varepsilon{\stackrel{\tau_{i}}{\longrightarrow}}_{\mathfrak{A}_{\psi}} p_{i} \\
& \text { and } r_{1} \ldots r_{m} \stackrel{\varphi}{\rightarrow}_{\mathfrak{A}_{\mathrm{TRACK}}} r_{0} \text { and } \forall 1 \leq i \leq m \cdot \varepsilon \stackrel{\tau_{i}}{\rightarrow} \mathfrak{A}_{\mathrm{TRACK}} r_{i} \\
& \text { and } q_{0}=q_{1}=\ldots=q_{m}=0 \\
& \Leftrightarrow \quad[\text { Definition of } \Delta] \\
& \exists\left(p_{1}, q_{1}, r_{1}\right), \ldots,\left(p_{m}, q_{m}, r_{m}\right) \in Q . \\
& \text { and }\left(p_{1}, q_{1}, r_{1}\right) \ldots\left(p_{m}, q_{m}, r_{1}\right) \stackrel{\varphi}{\rightarrow}_{\mathfrak{A}_{\exists z . \psi}}\left(p_{0}, q_{0}, r_{0}\right) \\
& \text { and } \forall 1 \leq i \leq m \cdot \varepsilon \stackrel{\tau_{i}}{\rightarrow} \mathfrak{A}_{\mathrm{TRACK}}\left(p_{i}, q_{i}, r_{i}\right) \text {. }
\end{aligned}
$$

The case $q_{0}=\ell>0$

$$
\begin{aligned}
& \stackrel{\tau}{\rightarrow}_{\mathfrak{A}_{\exists z \cdot \psi}}\left(p_{0}, \ell, r_{0}\right) \\
& \Leftrightarrow \quad {[\text { Definition of } \Delta \text { (only third case applicable) }] } \\
& \exists y \in \operatorname{Var}(\tau) \cdot \varepsilon \stackrel{(\tau[x \downarrow \ell]):\{x=y\}}{\longrightarrow} \mathfrak{A}_{\psi} p_{0} \\
& \text { and } \varepsilon \stackrel{(\tau[x \downarrow \ell]):\{x=y\}}{\longrightarrow} \mathfrak{A}_{\mathrm{TRACK}} r_{0}
\end{aligned}
$$

Now, since $\operatorname{Var}(\tau)$ can be partitioned into $\operatorname{Var}(\varphi)$ and, for each $1 \leq k \leq m$, $\operatorname{Var}\left(\tau_{k}\right) \backslash \operatorname{Var}(\varphi)$, variable $y$ occurs in exactly one of these sets. First, assume $y \in \operatorname{Var}(\varphi)$. Then

$$
\begin{aligned}
&(\dagger) \Leftrightarrow {[y \in \operatorname{Var}(\varphi)] } \\
& \exists y \in \operatorname{Var}(\varphi) \cdot \varepsilon \stackrel{(\tau[x \downarrow \ell]):\{x=y\}}{\rightarrow} \mathfrak{A}_{\psi} p_{0} \\
& \text { and } \varepsilon \stackrel{(\tau[x \downarrow \ell]):\{x=y\}}{\longrightarrow} \mathfrak{A}_{\mathrm{TRACK}} r_{0} \\
& \Leftrightarrow \quad {\left[\operatorname{Compositionality~of~} \mathfrak{A}_{\psi}, \mathfrak{A}_{\mathrm{TRACK}}\right] } \\
& \exists y \in \operatorname{Var}(\varphi) \cdot \exists p_{1}, \ldots, p_{m} \in Q_{\mathfrak{A}_{\psi}} \cdot \exists r_{1}, \ldots, r_{m} \in Q_{\mathfrak{A}_{\mathrm{TRACK}}} . \\
& p_{1} \ldots p_{m} \stackrel{(\varphi[x \downarrow \ell]):\{x=y\}}{\longrightarrow} \mathfrak{A}_{\psi} p_{0} \\
& \text { and } \forall 1 \leq i \leq m \cdot \varepsilon{\stackrel{\tau_{i}}{\longrightarrow}}_{\mathfrak{A}_{\psi}} p_{i} \\
& \text { and } r_{1} \ldots r_{m} \stackrel{(\varphi[x \downarrow \ell]):\{x=y\}}{\longrightarrow} \mathfrak{A}_{\mathrm{TRACK}} r_{0} \\
& \text { and } \forall 1 \leq i \leq m \cdot \varepsilon{\stackrel{\tau_{i}}{\longrightarrow}}_{\mathfrak{A}_{\mathrm{TRACK}}} r_{i}
\end{aligned}
$$




$$
\begin{aligned}
& \Leftrightarrow \quad\left[\text { choose } q_{1}=\ldots=q_{m}=0\right] \\
& \exists y \in \operatorname{Var}(\varphi) . \exists p_{1}, \ldots, p_{m} \in Q_{\mathfrak{A}_{\psi}} . \exists r_{1}, \ldots, r_{m} \in Q_{\mathfrak{A}_{\text {TRсСК }}} . \\
& p_{1} \ldots p_{m} \stackrel{(\varphi[x \downarrow \ell]):\{x=y\}}{\longrightarrow} \mathfrak{A}_{\psi} p_{0} \\
& \text { and } \forall 1 \leq i \leq m \cdot \varepsilon{\stackrel{\tau_{i}}{\longrightarrow}}_{\mathfrak{A}_{\psi}} p_{i} \\
& \text { and } r_{1} \ldots r_{m} \stackrel{(\varphi[x \downarrow \ell]):\{x=y\}}{\longrightarrow} \mathfrak{A}_{\text {TRACK }} r_{0} \\
& \text { and } \forall 1 \leq i \leq m \cdot \varepsilon \stackrel{\tau_{i}}{\rightarrow} \mathfrak{A}_{\mathrm{TRACK}} r_{i} \\
& \text { and } q_{1}=\ldots=q_{m}=0 \\
& \Leftrightarrow[\exists x \exists y \equiv \exists y \exists x \text {, Definition of } Q] \\
& \exists\left(p_{1}, q_{1}, r_{1}\right), \ldots,\left(p_{m}, q_{m}, r_{m}\right) \in Q . \exists y \in \operatorname{Var}(\varphi) . \\
& p_{1} \ldots p_{m} \stackrel{(\varphi[x \downarrow \ell]):\{x=y\}}{\longrightarrow} \mathfrak{A}_{\psi} p_{0} \\
& \text { and } \forall 1 \leq i \leq m \cdot \varepsilon \stackrel{\tau_{i}}{\longrightarrow} \mathfrak{A}_{\psi} p_{i} \\
& \text { and } r_{1} \ldots r_{m} \stackrel{(\varphi[x \downarrow \ell]):\{x=y\}}{\longrightarrow} \mathfrak{A}_{\text {TRACK }} r_{0} \\
& \text { and } \forall 1 \leq i \leq m \cdot \varepsilon \stackrel{\tau_{i}}{\rightarrow} \mathfrak{A}_{\text {TRACK }} r_{i} \\
& \text { and } q_{1}=\ldots=q_{m}=0 \\
& \Leftrightarrow[\text { Definition } \Delta, y \in \operatorname{Var}(\varphi)] \\
& \exists\left(p_{1}, q_{1}, r_{1}\right), \ldots,\left(p_{m}, q_{m}, r_{m}\right) \in Q . \\
& \left(p_{1}, q_{1}, r_{1}\right) \ldots\left(p_{m}, q_{m}, r_{m}\right) \stackrel{\varphi}{\rightarrow}_{\mathfrak{A}_{\exists z . \psi}}\left(p_{0}, q_{0}, r_{0}\right) \\
& \text { and } \forall 1 \leq i \leq m \cdot \varepsilon{\stackrel{\tau_{i}}{\longrightarrow}}_{\mathfrak{A}_{\exists}, \psi}\left(p_{i}, r_{i}, q_{i}\right) \\
& \text { and } q_{1}=\ldots=q_{m}=0 \\
& \Rightarrow[A \wedge B \rightarrow A] \\
& \exists\left(p_{1}, q_{1}, r_{1}\right), \ldots,\left(p_{m}, q_{m}, r_{m}\right) \in Q . \\
& \left(p_{1}, q_{1}, r_{1}\right) \ldots\left(p_{m}, q_{m}, r_{m}\right) \stackrel{\varphi}{\rightarrow}_{\mathfrak{A}_{\exists z . \psi}}\left(p_{0}, q_{0}, r_{0}\right) \\
& \text { and } \forall 1 \leq i \leq m \cdot \varepsilon{\stackrel{\tau_{i}}{\rightarrow}}_{\mathfrak{A}_{\exists z . \psi}}\left(p_{i}, r_{i}, q_{i}\right) \\
& \text { and } q_{1}=\ldots=q_{m}=0 \text {. }
\end{aligned}
$$

Note that equivalence holds up to the step marked with (\$) only. The last step works is only in one direction. We deal with the backwards direction after considering the second case. Thus assume $y \in \operatorname{Var}\left(\tau_{j}\right) \backslash \operatorname{Var}(\varphi)$ for some $1 \leq j \leq$ $m$. Then

$$
\begin{aligned}
&(\dagger) \Leftrightarrow {\left[y \in \operatorname{Var}\left(\tau_{j}\right) \backslash \operatorname{Var}(\varphi)\right] } \\
& \exists y \in \operatorname{Var}\left(\tau_{j}\right) \backslash \operatorname{Var}(\varphi) \cdot \varepsilon \stackrel{(\tau[x \downarrow \ell]):\{x=y\}}{\longrightarrow}_{\mathfrak{A}_{\psi}} p_{0} \\
& \quad \text { and } \varepsilon \stackrel{(\tau[x \downarrow \ell]):\{x=y\}}{\longrightarrow} \mathfrak{A}_{\mathrm{TRACK}} r_{0} \\
& \Leftrightarrow \quad\left[\text { Compositionality of } \mathfrak{A}_{\psi}, \mathfrak{A}_{\mathrm{TRACK}}\right] \\
& \exists \ell^{\prime} . \exists y \in \operatorname{Var}\left(\tau_{j}\right) \backslash \operatorname{Var}(\varphi) \cdot \exists p_{1}, \ldots, p_{m} \in Q_{\mathfrak{A}_{\psi}} .
\end{aligned}
$$




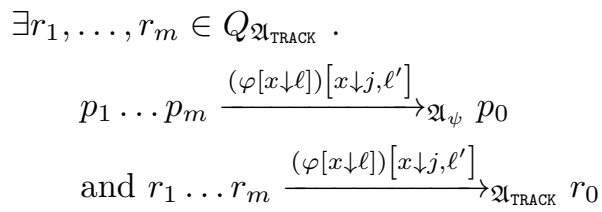

and $\forall 1 \leq i \leq m . i \neq j$ implies

$$
\varepsilon \stackrel{\tau_{i}}{\rightarrow} \mathfrak{A}_{\psi} p_{i} \text { and } \varepsilon \stackrel{\tau_{i}}{\rightarrow} \mathfrak{A}_{\mathrm{TRACK}} r_{i}
$$

and $\varepsilon \stackrel{\tau_{j}\left[x \downarrow \ell^{\prime}\right]}{\longrightarrow} \mathfrak{A}_{\psi} p_{j}$ and $\varepsilon \stackrel{\tau_{j}\left[x \downarrow \ell^{\prime}\right]}{\longrightarrow} \mathfrak{A}_{\psi} r_{j}$

$\Leftrightarrow \quad\left[\right.$ choose $q_{j}$ and for each $i \neq j$ choose $\left.q_{i}=0\right]$

$\exists y \in \operatorname{Var}\left(\tau_{j}\right) \backslash \operatorname{Var}(\varphi) . \exists p_{1}, \ldots, p_{m} \in Q_{\mathfrak{A}_{\psi}}$.

$\exists r_{1}, \ldots, r_{m} \in Q_{\mathfrak{A}_{\mathrm{TRACK}}} . \exists q_{1}, \ldots, q_{m} \in\{0, \ldots, \alpha\}$

$p_{1} \ldots p_{m} \stackrel{(\varphi[x \downarrow \ell])\left[x \downarrow j, q_{j}\right]}{\longrightarrow} \mathfrak{A}_{\psi} p_{0}$

and $r_{1} \ldots r_{m} \stackrel{(\varphi[x \downarrow \ell])\left[x \downarrow j, q_{j}\right]}{\longrightarrow} \mathfrak{A}_{\mathrm{TRACK}} r_{0}$

and $\forall 1 \leq i \leq m . i \neq j$ implies

$$
\varepsilon{\stackrel{\tau_{i}}{\longrightarrow}}_{\mathfrak{A}_{\psi}} p_{i} \text { and } \varepsilon{\stackrel{\tau_{i}}{\longrightarrow}}_{\mathfrak{A}_{\mathrm{TRACK}}} r_{i}
$$

and $\varepsilon \stackrel{\tau_{j}\left[x \downarrow q_{j}\right]}{\longrightarrow} \mathfrak{A}_{\psi} p_{j}$ and $\varepsilon \stackrel{\tau_{j}\left[x \downarrow q_{j}\right]}{\longrightarrow} \mathfrak{A}_{\psi} r_{j}$

and $q_{j}>0$ and $\forall i \neq j \cdot q_{i}=0$

$\Leftrightarrow[\exists x \exists y \equiv \exists y \exists x$, Definition of $Q]$

$\exists\left(p_{1}, q_{1}, r_{1}\right), \ldots,\left(p_{m}, q_{m}, r_{m}\right) \in Q$.

$\exists y \in \operatorname{Var}\left(\tau_{j}\right) \backslash \operatorname{Var}(\varphi)$.

$p_{1} \ldots p_{m} \stackrel{(\varphi[x \downarrow \ell])\left[x \downarrow j, q_{j}\right]}{\longrightarrow} \mathfrak{A}_{\psi} p_{0}$

and $r_{1} \ldots r_{m} \stackrel{(\varphi[x \downarrow \ell])\left[x \downarrow j, q_{j}\right]}{\longrightarrow} \mathfrak{A}_{\mathrm{TRACK}} r_{0}$

and $\forall 1 \leq i \leq m . i \neq j$ implies

$$
\varepsilon \stackrel{\tau_{i}}{\rightarrow} \mathfrak{A}_{\psi} p_{i} \text { and } \varepsilon \stackrel{\tau_{i}}{\longrightarrow} \mathfrak{A}_{\mathrm{TRACK}} r_{i}
$$

and $\varepsilon \stackrel{\tau_{j}\left[x \downarrow q_{j}\right]}{\longrightarrow} \mathfrak{A}_{\psi} p_{j}$ and $\varepsilon \stackrel{\tau_{j}\left[x \downarrow q_{j}\right]}{\longrightarrow} \mathfrak{A}_{\psi} r_{j}$

and $q_{j}>0$ and $\forall i \neq j . q_{i}=0$

$\Leftrightarrow \quad[$ Definition of $\Delta$ (second case for $\varphi)]$

$\exists\left(p_{1}, q_{1}, r_{1}\right), \ldots,\left(p_{m}, q_{m}, r_{m}\right) \in Q$.

$\left(p_{1}, q_{1}, r_{1}\right) \ldots\left(p_{m}, q_{m}, r_{m}\right) \stackrel{\varphi}{\rightarrow}_{\mathfrak{A}_{\exists z . \psi}}\left(p_{0}, q_{1}, r_{1}\right)$

and $\forall 1 \leq i \leq m \cdot \varepsilon \stackrel{\tau_{i}}{\rightarrow} \mathfrak{A}_{\exists z . \psi}\left(p_{i}, q_{i}, r_{i}\right)$

and $q_{j}>0$ and $\forall i \neq j . q_{i}=0$

$\Rightarrow[A \wedge B \rightarrow A]$

$\exists\left(p_{1}, q_{1}, r_{1}\right), \ldots,\left(p_{m}, q_{m}, r_{m}\right) \in Q$.

$\left(p_{1}, q_{1}, r_{1}\right) \ldots\left(p_{m}, q_{m}, r_{m}\right) \stackrel{\varphi}{\rightarrow}_{\mathfrak{A}_{\exists z . \psi}}\left(p_{0}, q_{1}, r_{1}\right)$ 


$$
\text { and } \forall 1 \leq i \leq m \cdot \varepsilon \stackrel{\tau_{i}}{\rightarrow} \mathfrak{A}_{\exists z . \psi}\left(p_{i}, q_{i}, r_{i}\right) .
$$

Again, the last step is in one direction only. It remains to show the converse direction. Assume, for $q_{0}=\ell>0$,

$$
\begin{aligned}
& \exists\left(p_{1}, q_{1}, r_{1}\right), \ldots,\left(p_{m}, q_{m}, r_{m}\right) \in Q . \\
& \quad\left(p_{1}, q_{1}, r_{1}\right) \ldots\left(p_{m}, q_{m}, r_{m}\right) \stackrel{\varphi}{\rightarrow} \mathfrak{A}_{\exists z . \psi}\left(p_{0}, q_{1}, r_{1}\right) \\
& \quad \text { and } \forall 1 \leq i \leq m . \varepsilon \stackrel{\tau_{i}}{\rightarrow} \mathfrak{A}_{\exists z . \psi}\left(p_{i}, q_{i}, r_{i}\right) .
\end{aligned}
$$

Then, by construction of $\Delta$ either $q_{1}=q_{2}=\ldots=q_{m}=0$ or exactly one $q_{j}>0$, for some $1 \leq j \leq m$. If $q_{1}=q_{2}=\ldots=q_{m}=0$ then only the third case of $\Delta$ is applicable to $\varphi$ (and there exists some suitable $y \in \operatorname{Var}(\varphi)$ ). Thus, (⿻) holds. As shown before, this is equivalent to $\varepsilon \stackrel{\tau}{\rightarrow}_{\mathfrak{A}_{\text {TRACK }}} q_{0}$. If there exists exactly one $q_{j}$ with $q_{j}>0$ then $(\mathbf{)})$ holds. As shown above, this case is equivalent to $\varepsilon \stackrel{\tau}{\rightarrow}_{\mathfrak{A}_{\text {TRAск }}} q_{0}$. Hence, in each case, $\mathfrak{A}_{\exists z . \psi}$ satisfies the compositionality property.

Lemma 47. $L\left(\mathfrak{A}_{\exists z . \psi}\right)=H_{\exists x . \psi, \Phi}^{\langle\alpha\rangle}$, where $x=\mathbf{x}_{0}^{\psi}[k]$ for some fixed position $k$.

Proof. Let $\tau \in \mathrm{RSH}_{\langle\alpha\rangle}$. Then

$$
\begin{aligned}
& \tau \in L\left(\mathfrak{A}_{\exists z . \psi}\right) \\
& \Leftrightarrow \quad\left[\text { Definition } L\left(\mathfrak{A}_{\exists z . \psi}\right)\right] \\
& \exists\left(p_{0}, q_{0}, r_{0}\right) \in F . \varepsilon \stackrel{\tau}{\rightarrow}_{\mathfrak{A}_{\exists z}, \psi}\left(p_{0}, q_{0}, r_{0}\right) \\
& \Leftrightarrow \text { [Definition of } F] \\
& \exists p_{0} \in F_{\mathfrak{A}_{\psi}} \cdot \exists r_{0} \in Q_{\mathfrak{A}_{\text {тRAсK }}} \cdot \varepsilon \stackrel{\tau}{\rightarrow}_{\mathfrak{A}_{\text {тRAск }}}\left(p_{0}, k, r_{0}\right) \\
& \Leftrightarrow \text { [Definition of } \Delta \text { (only third case is applicable)] } \\
& \exists p_{0} \in F_{\mathfrak{A}_{\psi}} . \exists r_{0} \in Q_{\mathfrak{A}_{\text {тRACK }}} . \exists y \in \operatorname{Var}(\tau) . \\
& \varepsilon \stackrel{\tau[x \downarrow k]:\{y=x\}}{\longrightarrow} \mathfrak{A}_{\psi} p_{0} \text { and } \varepsilon \stackrel{\tau[x \downarrow k]:\{y=x\}}{\longrightarrow} \mathfrak{A}_{\mathrm{TRACK}} r_{0} \\
& \Leftrightarrow[\exists x \exists y \equiv \exists y \exists x] \\
& \exists y \in \operatorname{Var}(\tau) . \exists p_{0} \in F_{\mathfrak{A}_{\psi}} . \exists r_{0} \in Q_{\mathfrak{A}_{\text {TRACK }}} . \\
& \varepsilon \stackrel{\tau[x \downarrow k]:\{y=x\}}{\longrightarrow} \mathfrak{A}_{\psi} p_{0} \text { and } \varepsilon \stackrel{\tau[x \downarrow k]:\{y=x\}}{\longrightarrow} \mathfrak{A}_{\text {TRACK }} r_{0} \\
& \Leftrightarrow \quad \text { for each } \sigma \text { there exists a } r_{0} \in Q_{\mathfrak{A}_{\text {TRACK }}} \text { ] } \\
& \exists y \in \operatorname{Var}(\tau) . \exists p_{0} \in F_{\mathfrak{A}_{\psi}} \cdot \varepsilon \stackrel{\tau[x \downarrow k]:\{y=x\}}{\longrightarrow} \mathfrak{A}_{\psi} p_{0} \\
& \left.\Leftrightarrow \quad \text { [Definition of } L\left(\mathfrak{A}_{\psi}\right)\right] \\
& \exists y \in \operatorname{Var}(\tau) .(\tau[x \downarrow k]:\{y=x\}) \in L\left(\mathfrak{A}_{\psi}\right) \\
& \Leftrightarrow\left[L\left(\mathfrak{A}_{\psi}\right)=H_{\psi, \Phi}^{\langle\alpha\rangle}\right] \\
& \exists y \in \operatorname{Var}(\tau) . \tau[x \downarrow k]:\{y=x\} \models_{\Phi} \psi \\
& \Leftrightarrow \text { [Lemma 45] } \\
& \tau \models_{\Phi} \exists x . \psi
\end{aligned}
$$




$$
\begin{gathered}
\Leftrightarrow \quad\left[\text { Definition of } H_{\exists x . \psi, \Phi}^{\langle\alpha\rangle}\right] \\
\tau \in H_{\exists x . \psi, \Phi}^{\langle\alpha\rangle} .
\end{gathered}
$$

\section{A.27 Complexity of Entailment}

This section provides a detailed complexity analysis of Algorithm 2. In particular, this includes a proof of Theorem 12

\section{A.27.1 Upper Complexity Bound for Entailments}

For the remainder of this section, we fix some notation used in Algorithm 2: Let $\varphi, \mathfrak{A}_{\psi}$, etc., be as in Algorithm 2. Further, let $\Phi$ be an SID such that the unfoldings of each predicate call can be accepted by a heap automaton over $\langle\alpha\rangle$. Moreover, let $k \triangleq\|\Phi\|+\|\varphi\|+\|\psi\|$ and $M \leq k$ denote the maximal number of predicate calls in $\varphi$ and any symbolic heap in $\Phi$.

We first analyze run-time of Algorithm 2 for arbitrary SIDs.

Lemma 48. Algorithm 2 decides whether $\varphi \models_{\Phi} \psi$ holds in

$$
\mathcal{O}\left(2^{\text {poly }(k)} \cdot\left(2^{\left\|Q_{\mathfrak{A}_{\psi}}\right\|}\right)^{2(M+1)} \cdot\left\|\Delta_{\mathfrak{A}_{\psi}}\right\|\right),
$$

where poly $(k)$ denotes some polynomial function in $k$.

Proof. Our previous complexity analysis of Algorithm 1 reveals that $\mathbb{U}_{\Psi}(P \mathbf{x}) \cap$ $L\left(\overline{\mathfrak{A}_{\psi}}\right)=\emptyset$ is decidable in

$$
\mathcal{O}\left(\|\Psi\| \cdot\left\|Q_{\overline{\mathfrak{A}_{\psi}}}\right\|^{M+1} \cdot\left\|\Delta_{\overline{\mathfrak{A}_{\psi}}}\right\|\right) .
$$

Regarding $\|\Psi\|$, applying the Refinement Theorem (Theorem 1) to $\Phi \cup\{P \Leftarrow \varphi\}$ and $\mathfrak{A}_{\mathrm{SAT}}$ (cf. Theorem 3) yields an SID $\Psi$ of size

$$
\|\Psi\| \leq c \cdot\|\Phi\| \cdot 2^{\|\varphi\|^{2}} \cdot 2^{2 \alpha^{2}+\alpha} \leq 2^{\text {poly }(k)},
$$

for some positive constant $c$. Then $\Psi$ is computable in $\mathcal{O}\left(2^{\text {poly }(k)}\right)$. Furthermore, $\overline{\mathfrak{A}}_{\psi}$ is obtained from complementation of $\mathfrak{A}_{\psi}$. Thus, by the construction to prove Lemma 2, we obtain that $\left\|Q_{\overline{\mathfrak{A}_{\psi}}}\right\| \leq 2^{\left\|Q_{\mathfrak{A}_{\psi}}\right\|}$ and that $\Delta_{\overline{\mathfrak{A}_{\psi}}}$ is decidable in $\left(2^{\left\|Q_{\mathfrak{A}_{\psi}}\right\|}\right)^{M+1} \cdot\left\|\Delta_{\mathfrak{A}_{\psi}}\right\|$. Putting both into (\$) yields the result.

Towards a more fine-grained analysis, recall from Definition 14 our assumption that SIDs are $\alpha$-bounded. Further, we assume the arity of points-to assertions $x \mapsto \mathbf{y}$, i.e., $\|\mathbf{y}\|$, to be bounded by some $\gamma \geq 0$. Our next observation is that heap automata constructed for arbitrary determined symbolic heaps according to Theorem 10 satisfy the same constraints. Formally, 
Lemma 49. Let $\Phi$ be an $\alpha$-bounded $S I D$ and $\psi \in \mathrm{SH}_{\langle\alpha\rangle}^{\Phi}$. Then a heap automaton $\mathfrak{A}_{\psi}$ accepting $H_{\psi, \Phi}^{\langle\alpha\rangle}$ can be constructed such that $\Delta_{\mathfrak{A}_{\psi}}$ is decidable in $\mathcal{O}\left(2^{\text {poly }(k)}\right)$ and $\left\|Q_{\mathfrak{A}_{\psi}}\right\| \leq 2^{\text {poly }(\alpha)}$.

Proof. By induction on the structure of symbolic heaps, we show $\alpha$-boundedness for the heap automata constructed in the proof of Theorem 10.

We are now in a position to derive an upper complexity bound on the entailment problem for a permissive symbolic heap fragment of separation logic with inductive predicate definitions.

Lemma 50. SL-ENTAIL ${ }_{\langle\alpha\rangle}^{\Phi}$ is decidable in 2-EXPTIME for each $\alpha$-bounded SID $\Phi$.

Proof. By Lemma 48, we know that an entailment $\varphi \models_{\Phi} \psi$ can be discharged in $\mathcal{O}\left(2^{\text {poly }(k)} \cdot\left(2^{\left\|Q_{\mathfrak{A}_{\psi}}\right\|}\right)^{2(M+1)} \cdot\left\|\Delta_{\mathfrak{A}_{\psi}}\right\|\right)$. By Lemma $49, \Delta_{\mathfrak{A}_{\psi}}$ is decidable in $\mathcal{O}\left(2^{\text {poly }(k)}\right)$ and $\left\|Q_{\mathfrak{A}_{\psi}}\right\| \leq 2^{\text {poly }(\alpha)}$. Then it is easy to verify that $\varphi \models_{\Phi} \psi$ is decidable in $\mathcal{O}\left(2^{2^{\text {poly }(k)}}\right)$ :

$$
\begin{aligned}
& \mathcal{O}\left(\|\Psi\| \cdot\left\|Q_{\overline{\mathfrak{A}_{\psi}}}\right\|^{M+1} \cdot\left\|\Delta_{\overline{\mathfrak{A}_{\psi}}}\right\|\right) \\
& =\left[\|\Psi\| \leq 2^{\text {poly }(k)},\left\|Q_{\overline{\mathfrak{A}_{\psi}}}\right\| \leq 2^{\| Q_{\mathfrak{A} \psi}} \|, M \leq 2 k\right] \\
& \mathcal{O}\left(2^{\text {poly }(k)} \cdot\left(2^{\left\|Q_{\mathfrak{A}_{\psi}}\right\|}\right)^{2 k} \cdot\left\|\Delta_{\overline{\mathfrak{A}_{\psi}}}\right\|\right) \\
& =\left[\left\|\Delta_{\mathfrak{A}_{\psi}}\right\| \leq\left(2^{\left\|Q_{\mathfrak{A}_{\psi}}\right\|}\right)^{M+1} \cdot\left\|\Delta_{\mathfrak{A}_{\psi}}\right\|\right] \\
& \mathcal{O}\left(2^{\text {poly }(k)} \cdot\left(2^{\left\|Q_{\mathfrak{A}_{\psi}}\right\|}\right)^{4 k} \cdot\left\|\Delta_{\mathfrak{A}_{\psi}}\right\|\right) \\
& =\left[\left\|Q_{\mathfrak{A}_{\psi}}\right\| \leq 2^{\text {poly }(k)}\right] \\
& \mathcal{O}\left(2^{\text {poly }(k)} \cdot\left(2^{2^{\text {poly }(k)}}\right)^{4 k} \cdot\left\|\Delta_{\mathfrak{A}_{\psi}}\right\|\right) \\
& =\left[\left(a^{b}\right)^{c}=a^{b c},\left\|\Delta_{\mathfrak{A}_{\psi}}\right\| \in \mathcal{O}\left(2^{\text {poly }(k)}\right)\right] \\
& \mathcal{O}\left(2^{\mathrm{poly}(k)} \cdot 2^{4 k \cdot 2^{\mathrm{poly}(k)}} \cdot 2^{\mathrm{poly}(k)}\right) \\
& =\mathcal{O}\left(2^{2^{\text {poly }(k)}}\right)
\end{aligned}
$$

Hence, SL-ENTAIL ${ }_{\langle\alpha\rangle}^{\Phi}$ is in 2-EXPTIME.

Since the maximal arity $\alpha$ of predicate symbols is fixed for any given SID, we also analyze Algorithm 2 under the assumption that $\alpha$ is bounded by a constant. This is a common assumption (cf. $[11,13])$ that was considered in Section 4 already. 
Lemma 51. Let $\Phi$ be an $\alpha$-bounded SID for some constant $\alpha \geq 1$. Then the entailment problem SL-ENTAIL ${ }_{\langle\alpha\rangle}^{\Phi}$ is in EXPTIME.

Proof. By Lemma $49,\left\|Q_{\mathfrak{A}_{\psi}}\right\| \leq 2^{\text {poly }(\alpha)}$ and $\Delta_{\mathfrak{A}_{\psi}}$ is decidable in $\mathcal{O}\left(2^{\text {poly }(k)}\right)$. Since $\alpha$ is bounded by a constant, so is $\left\|Q_{\mathfrak{A}_{\psi}}\right\|$. Then, by Lemma 48 , we know that SL-ENTAIL $L_{\langle\alpha\rangle}^{\Phi}$ is decidable in

$$
\mathcal{O}\left(2^{\text {poly }(k)} \cdot\left(2^{\left\|Q_{\mathfrak{A}_{\psi}}\right\|}\right)^{2(M+1)} \cdot 2^{\mathrm{poly}(k)}\right)=\mathcal{O}\left(2^{\mathrm{poly}(k)}\right),
$$

which clearly is in ExpTime.

Then, the upper complexity bounds provided in Theorem 12 hold by Lemma 50 and Lemma 51. Further, ExpTime-completeness follows directly from[1, Theorem 5] and Appendix A.27.2.

\section{A.27.2 Lower Complexity Bound for Entailments}

The proof of the ExPTIME-lower bound in [1] is by reducing the inclusion problem for nondeterministic finite tree automata (NFTA, cf. [18]) to the entailment problem. Their proof requires a constant (or free variable) for each symbol in the tree automatons alphabet. In contrast, we prove their result by encoding the alphabet in a null-terminated singly-linked list. Thus, a tree $a(b, a(b, b)$ is encoded by a reduced symbolic heap

$$
\begin{aligned}
& \exists z_{1} z_{2} z_{3} z_{4} z_{5} z_{6} z_{7} . \\
& \quad x \mapsto\left(z_{1} z_{2} \text { null }\right) \\
& \quad * z_{1} \mapsto\left(\text { null null } z_{3}\right) * z_{3} \mapsto(\text { null null null }) \\
& \quad * z_{2} \mapsto\left(z_{4} z_{5} \text { null }\right) \\
& \quad * z_{4} \mapsto\left(\text { null null } z_{6}\right) * z_{6} \mapsto(\text { null null null }) \\
& \quad * z_{5} \mapsto\left(\text { null null } z_{7}\right) * z_{7} \mapsto(\text { null null null }),
\end{aligned}
$$

where the symbol $a$ is encoded by having null as third component in a points-to assertion and symbol $b$ by a null terminated list of length one.

Now, given some NFTA $\mathfrak{T}=(Q, \Sigma, \Delta, F)$ with $\Sigma=\left\{a_{1}, \ldots, a_{n}\right\}$, we construct a corresponding $\Phi$. Without less of generality, we assume that $\mathfrak{T}$ contains no unreachable or unproductive states. We set $\operatorname{Pred}(\Phi) \triangleq Q \cup \Sigma \cup\{I\}$, where each predicate symbol is of arity one. Then, for each symbol $a_{i} \in \Sigma$ one rule of the form $a_{1} \Leftarrow \mathbf{x}_{0}[1]=$ null or, for $1<i \leq n$,

$$
\begin{aligned}
a_{i} \Leftarrow \exists z_{1} & z_{2} \ldots z_{i-1} \cdot \mathbf{x}_{0} 1[\mapsto]\left(\text { null null } z_{1}\right) \\
* \star_{1 \leq j<i} z_{j} & \mapsto\left(\text { null null } z_{j+1}\right):\left\{z_{i-1}=\text { null }\right\}
\end{aligned}
$$

is added to $\Phi$. Furthermore, for each $\left(p_{1} \ldots p_{m}, a_{i}, p_{0}\right) \in \Delta, 1 \leq i \leq n$, we add a rule

$$
p_{0} \Leftarrow \exists z_{1} \ldots z_{m+1} \cdot \mathbf{x}_{0}[1] \mapsto\left(z_{1} \ldots z_{m+1}\right)
$$




$$
* a_{i}\left(z_{m+1}\right) * \star_{1 \leq i \leq m} p_{i}\left(z_{i}\right) .
$$

Finally, we add rules $I \Leftarrow p \mathbf{x}_{0}[1]:\left\{\mathbf{x}_{0}[1] \neq\right.$ null $\}$ for each $p \in F$. Clearly $\Phi$ is established. Moreover, it is easy to verify that, given two NFTAs $\mathfrak{T}_{1}$ and $\mathfrak{T}_{2}$ with distinct sets of states, we have

$$
I_{1} x \models_{\Phi_{1} \cup \Phi_{2}} I_{2} x \text { iff } L\left(\mathfrak{T}_{1}\right) \subseteq L\left(\mathfrak{T}_{2}\right) .
$$

Thus, following [1], if $H_{I x, \Phi}^{\langle 1\rangle}$ can be accepted by a heap automaton, the entailment problem SL-ENTAIL ${ }_{\Phi}^{\langle\alpha\rangle}$ is EXPTIME-hard for certain SIDs $\Phi$ fixed $\alpha=1$, and a fixed arity of points-to assertions $\gamma=3$. Such a heap automaton can easily be constructed. Formally, let $\mathfrak{T}=(Q, \Sigma, \Delta, F)$ be an NFTA as above and $Q=\left\{p_{1}, \ldots, p_{k}\right\}$ for some $k>0$. Furthermore, for each state $p_{i}$, let $t_{i}$ be some fixed finite tree that is accepted by the tree automaton $\mathfrak{T}_{i}=\left(Q, \Sigma, \Delta,\left\{p_{i}\right\}\right)$ and $\tau_{i}$ be the corresponding encoding as a reduced symbolic heap. One possible (not necessarily efficient) heap automaton $\mathfrak{A}=\left(Q_{\mathfrak{A}}, \mathrm{SH}_{\langle 1\rangle}, \Delta_{\mathfrak{A}}, F_{\mathfrak{A}}\right)$ is given by:

$$
\begin{aligned}
& Q_{\mathfrak{A}} \triangleq\left\{\tau_{i} \mid 1 \leq i \leq k\right\} \cup\left\{a_{i} \mid 1 \leq i \leq n\right\} \\
& F_{\mathfrak{A}} \triangleq F \\
& q_{0} \stackrel{\varphi}{\rightarrow}{ }_{\mathfrak{A}} q_{1} \ldots q_{m} \text { iff } \varphi\left[P_{1} / q_{1}, \ldots, P_{m} / q_{m}\right] \models_{\Phi} P \mathbf{x}_{0}[1]
\end{aligned}
$$

where each $a_{i}$ corresponds to the reduced symbolic heap encoding symbol $a_{i}$ and $P$ is the predicate $p_{i}$ corresponding to reduced symbolic heap $\tau_{i}$ as previously described.

\section{A.28 Construction of Heap Automata for Entailment}

This section presents a systematic way to construct heap automata to discharge entailments. Further, we provide two example constructions.

\section{A.28.1 Systematic Construction of $\boldsymbol{H}_{\varphi, \Phi}^{\langle\alpha\rangle}$}

Using heap automata to discharge entailments as presented in Theorem 11 requires the construction of suitable heap automata for each predicate symbol of an SID. We briefly present a systematic construction of such heap automata that is similar to the well-known Myhill-Nerode Theorem for regular (tree) languages $[18,34]$ : We partition the set of all reduced symbolic heaps into equivalence classes, where two formulas belong to the same equivalence class if they can be extended in the same way to formulas entailing a predicate $P$ of interest.

Definition 25. Let $P \in \operatorname{Pred}(\Phi), \Phi \in \operatorname{SID}$. Then two symbolic heaps $\tau, \sigma \in$ $\mathrm{RSH}_{\langle\alpha\rangle}$ with $\left\|\mathbf{x}_{0}^{\tau}\right\|=\left\|\mathbf{x}_{0}^{\sigma}\right\|=\beta$ are $P$-equivalent, written $\tau \cong_{P} \sigma$, if for all $\varphi \in \mathrm{SH}$ with exactly one predicate call $I$ of arity $\beta$, we have $\varphi[I / \tau] \models_{\emptyset} P \mathbf{x}_{0}$ iff $\varphi[I / \sigma] \models_{\emptyset} P \mathbf{x}_{0}$. 
For example, all non-empty singly linked list segments of the sll predicate from Section 1 with $\mathbf{x}_{0}[1]$ as head and $\mathbf{x}_{0}[2]$ as tail are sll-equivalent.

Theorem 13. Let $P$ be a predicate symbol of an SID $\Phi$. Then there exists a heap automaton $\mathfrak{A}_{P}$ with $L\left(\mathfrak{A}_{P}\right)=H_{P \mathbf{x}_{0}, \Phi}^{\langle\alpha\rangle}$ iff the number of equivalence classes of $\cong_{P}$ is finite.

Proof. Assume there are only finitely many equivalence classes of $\cong_{P}$. Furthermore, let $[\tau]_{\bigwedge_{P}}$ denote the equivalence class containing formula $\tau$. Then a heap automaton $\mathfrak{A}_{P}=\left(Q, \mathrm{SH}_{\langle\alpha\rangle}, \Delta, F\right)$ accepting $H_{P \mathbf{x}_{0}, \Phi}^{\langle\alpha\rangle}$ is given by $Q=\left\{[\tau]_{\cong_{P}} \mid \tau \in\right.$ $\left.\operatorname{RSH}_{\langle\alpha\rangle}\right\}, F=\left\{[\tau]_{\cong_{P}} \mid \tau \in \mathrm{RSH}_{\langle\alpha\rangle}, \tau \models_{\Phi} P \mathbf{x}_{0}\right\}$ and

$$
\left[\tau_{1}\right]_{\cong_{P}} \ldots\left[\tau_{m}\right]_{\cong_{P}} \stackrel{\varphi}{\rightarrow}_{\mathfrak{A}_{P}}\left[\varphi\left[P_{1} \mathbf{x}_{1} / \tau_{1}, P_{m} \mathbf{x}_{m} / \tau_{m}\right]\right] \cong_{P}
$$

for each symbolic heap $\varphi \in \mathrm{SH}_{\langle\alpha\rangle}$ with $\left\|\Gamma^{\varphi}\right\|=m$. Then it is easy to verify that $\mathfrak{A}$ satisfies the compositionality property and $L\left(\mathfrak{A}_{P}\right)=H_{P \mathbf{x}_{0}, \Phi}^{\langle\alpha\rangle}$. The converse direction is straightforward. A full proof is found in [30].

Note that it suffices to represent each equivalence class by a single reduced symbolic heap, e.g. the smallest one. While the construction principle from above is generally applicable, it is, however, often preferable to exploit other properties of the predicates of interest, e.g., acyclicity (see Section 4.6), to reduce the number of equivalence classes.

\section{A.28.2 Example: Singly-linked List Segments}

Recall the SID for acyclic singly-linked list segments from Example 1. A heap automaton $\mathfrak{A}$ for sll is defined in Fig. 5 .

Observe that $\Delta$ is compositional. Note further that we have defined $\Delta$ in such way that $(\varepsilon, \tau, q) \in \Delta$ for $q \in F$ iff $\tau \models \operatorname{sll} \mathbf{x}_{0}$, i.e., $L(\mathfrak{A})=H_{\operatorname{sll} \mathbf{x}, \Phi}^{\langle\alpha\rangle}$.

Figure 5 also shows the canonical representations of each state, i.e., the minimal unfoldings of each state's formula. These are the symbolic heaps that are substituted into the predicate calls in symbolic heaps $\varphi$ to obtain simple entailment problems for deciding transitions $(\mathbf{q}, \varphi, q)$.

\section{A.28.3 Example: Trees with Linked Leaves}

We consider an example of the systematic construction of heap automata: We determine the equivalence classes $\cong_{t 11}$, where the SID tll is defined as in Example 1, to obtain a heap automaton for the set of well-determined reduced symbolic heaps that entail some tree with linked leaves (TLL). Note that this SID is outside the scope of previous decision procedures for entailment with (at most) exponential-time complexity $[3,28]$. 
$Q \triangleq\left\{q_{\mathrm{eq}}, q_{\mathrm{diff}}, q_{\mathrm{rev}}, q_{\mathrm{fst}}, q_{\mathrm{snd}}, q_{\perp}\right\}$

$$
F \triangleq\left\{q_{\mathrm{eq}}, q_{\mathrm{diff}}, q_{\mathrm{fst}}\right\}
$$

Transitions:

$$
\begin{aligned}
& \left(\varepsilon, \tau, q_{\mathrm{eq}}\right) \in \Delta \\
& \left(\varepsilon, \tau, q_{\mathrm{diff}}\right) \in \Delta \\
& \left(\varepsilon, \tau, q_{\mathrm{rev}}\right) \in \Delta \\
& \left(\varepsilon, \tau, q_{\mathrm{fst}}\right) \in \Delta \\
& \left(\varepsilon, \tau, q_{\mathrm{snd}}\right) \in \Delta \\
& \left(\varepsilon, \tau, q_{\perp}\right) \in \Delta \\
& (\mathbf{q}, \varphi, q) \in \Delta
\end{aligned}
$$

$$
\begin{aligned}
& \text { iff } \left.\tau \models \operatorname{emp}:\left\{\mathbf{x}_{0}[1]=\mathbf{x}_{0}[2]\right)\right\} \\
& \quad \text { or } \tau \models \operatorname{emp} \wedge\left\|\mathbf{x}_{0}^{\varphi}\right\|=2 \\
& \text { iff } \tau \models \operatorname{sll} \mathbf{x}_{0}[1] \mathbf{x}_{0}[2]:\left\{\mathbf{x}_{0}[2] \neq \text { null }\right\} \\
& \text { iff } \tau \models \operatorname{sll} \mathbf{x}_{0}[2] \mathbf{x}_{0}[1]:\left\{\mathbf{x}_{0}[1] \neq \mathbf{n u l l}\right\} \\
& \text { iff } \tau \models \operatorname{sll} \mathbf{x}_{0}[1] \mathbf{n u l l} \\
& \text { iff } \tau \models \operatorname{sll} \mathbf{x}_{0}[2] \mathbf{n u l l} \\
& \text { iff } \tau \not \models \operatorname{sll} \mathbf{x}_{0} \\
& \text { iff }\left(\varepsilon, \varphi\left[P_{1} / \rho_{\mathbf{q}[1]}, \ldots, P_{m} / \rho_{\mathbf{q}[m]}\right], q\right) \in \Delta
\end{aligned}
$$

\section{Representations:}

$\rho_{q_{\mathrm{eq}}} \triangleq \operatorname{emp}:\left\{\mathbf{x}_{0}[1]=\mathbf{x}_{0}[2]\right\}$

$\rho_{q_{\mathrm{diff}}} \triangleq \mathbf{x}_{0}[1] \mapsto\left(\mathbf{x}_{0}[2]\right):\left\{\mathbf{x}_{0}[2] \neq\right.$ null, $\left.\mathbf{x}_{0}[2] \neq \mathbf{x}_{0}[1]\right\}$

$\rho_{q_{\mathrm{rev}}} \triangleq \mathbf{x}_{0}[2] \mapsto\left(\mathbf{x}_{0}[1]\right):\left\{\mathbf{x}_{0}[1] \neq\right.$ null, $\left.\mathbf{x}_{0}[2] \neq \mathbf{x}_{0}[1]\right\}$

$\rho_{q_{\mathrm{fst}}} \triangleq \mathbf{x}_{0}[1] \mapsto($ null $)$

$\rho_{q_{\text {snd }}} \triangleq \mathbf{x}_{0}[2] \mapsto($ null $)$

$\rho_{q_{\perp}} \triangleq \mathbf{x}_{0}[1] \neq \mathbf{x}_{0}[1]$

Fig. 3. A heap automaton $\mathfrak{A}=(Q,\langle\alpha\rangle, \Delta, F)$ with $L(\mathfrak{A})=H_{\mathrm{s} 11 \mathbf{x}, \Phi}^{\langle\alpha\rangle}$, for acyclic singlylinked list fragments sll as defined in Ex. 1; plus canonical representations $\rho_{q}$ for each state $q$. 
For simplicity of presentation, we assume that all parameters of the tll predicate are different from null and consider only acyclic TLLs. ${ }^{22}$ Recall that we can always check whether these conditions are satisfied (see Sections 4.1, 4.6).

We will represent each $\cong_{t 11}$-equivalence class by an SID whose unfoldings are exactly the reduced symbolic heaps contained in the respective class. To this end, let core be a predicate specifying TLLs that lack the outgoing pointer of the right-most leaf:

$$
\begin{aligned}
\text { core } \Leftarrow & \operatorname{emp}:\left\{\mathbf{x}_{0}[1]=\mathbf{x}_{0}[2]\right\} \\
\text { core } \Leftarrow & \exists \ell r z \cdot \mathbf{x}_{0}[1] \mapsto(\ell r \text { null }) \\
& * \operatorname{tll}\left(\ell \mathbf{x}_{0}[2] z\right) * \operatorname{core}\left(r z \mathbf{x}_{0}[3]\right)
\end{aligned}
$$

Here, the omitted pointer is reflected by the missing points-to assertion in the base case. In the following, let $\mathcal{S}_{\mathbf{x}}$ be the set of all permutations of $\mathbf{x}_{0}$.

Example 11. For each $\rho \in \mathcal{S}_{\mathbf{x}}$, the relation $\cong_{\text {tll }}$ has 8 equivalence classes that can be extended to entail an unfolding of tll:

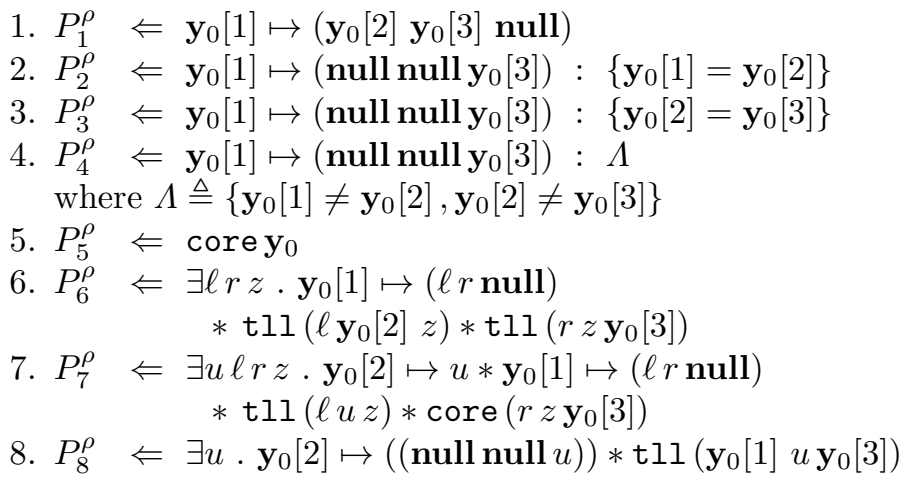

We refer the reader to Figure 4 for an illustration of the unfoldings covered by each predicate. Due to lack of space, this figure is found in the appendix. In addition, $\cong_{t 11}$ has one equivalence class of symbolic heaps that cannot be extended to entail tll unfoldings, defined as the complement of the other classes: $\mathrm{RSH}_{\mathrm{FV} \leq \alpha} \backslash \bigcup_{1 \leq i \leq 8, \rho \in \mathcal{S}_{\mathbf{x}}} P_{i}^{\rho}$

For example, $P_{7}^{\rho}$ specifies all trees with linked leaves that consist of more than one pointer, lack the successor of the rightmost leaf, but have an additional element at the head of the list of linked leaves. All such symbolic heaps can be extended to heaps that entail an unfolding of tll $\mathbf{x}_{0}$ in the same way; for example by adding the missing pointer from the last leaf to its successor and adding a new root node that points to the additional leaf at the head of the list

\footnotetext{
$\overline{22}$ The only parameter that can actually be null is $\mathbf{x}_{0}$ [3], as the other two parameters are always equal to a variable that appears on the left-hand side of a points-to assertion. Likewise, $\mathbf{x}_{0}[3]$ is the only parameter that can introduce a cycle - it can be equal to $\mathbf{x}_{0}[1]$ or $\mathbf{x}_{0}[2]$.
} 


$$
P_{1}^{\rho} \Leftarrow \mathbf{y}_{0}[1] \mapsto\left(\mathbf{y}_{0}[2] \mathbf{y}_{0}[3] \text { null }\right)
$$

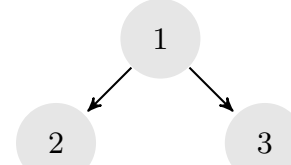

$$
\begin{aligned}
& P_{2}^{\rho} \Leftarrow \mathbf{y}_{0}[1] \mapsto\left(\text { null null } \mathbf{y}_{0}[3]\right):\left\{\mathbf{y}_{0}[1]=\mathbf{y}_{0}[2]\right\} \\
& P_{3}^{\rho} \Leftarrow \mathbf{y}_{0}[1] \mapsto\left(\text { null null } \mathbf{y}_{0}[3]\right):\left\{\mathbf{y}_{0}[2]=\mathbf{y}_{0}[3]\right\} \\
& P_{4}^{\rho} \Leftarrow \mathbf{y}_{0}[1] \mapsto\left(\text { null null } \mathbf{y}_{0}[3]\right): \Lambda
\end{aligned}
$$$$
1,2 \longrightarrow 3
$$$$
1 \longrightarrow 2,3
$$

$$
P_{5}^{\rho} \Leftarrow \operatorname{core} \mathbf{y}_{0}
$$

$$
P_{6}^{\rho} \Leftarrow \exists \ell r z \cdot \mathbf{y}_{0}[1] \mapsto(\ell r \text { null })
$$

$$
P_{7}^{\rho} \Leftarrow \exists u \ell r z \cdot \mathbf{y}_{0}[2] \mapsto(u) * \mathbf{y}_{0}[1] \mapsto(\ell r \text { null })
$$$$
P_{8}^{\rho} \Leftarrow \exists u \cdot \mathbf{y}_{0}[2] \mapsto(\text { null null } u) * \operatorname{tll} \mathbf{y}_{0}[1] u \mathbf{y}_{0}[3]
$$
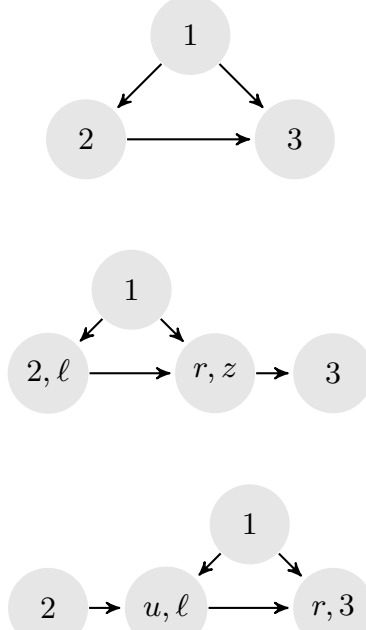

Fig. 4. Equivalence classes with graphical representatives of small unfoldings of each predicate. Here, numbers correspond to the index of tuple $\mathbf{y}$ and letters to the respective variables in the predicates. 
as well as to the root of the $P_{7}^{\rho}$-unfolding. Formally, for the identity permutation id $\in \mathcal{S}_{\mathbf{x}}$, let

$$
\varphi^{\mathrm{id}} \triangleq \exists u v \cdot \mathbf{x}_{0}[1] \mapsto\left(\mathbf{x}_{0}[2] u \text { null }\right) * P_{7}^{\text {id }}\left(u \mathbf{x}_{0}[2] v\right) * v \mapsto\left(\text { null null } \mathbf{x}_{0}[3]\right)
$$

Then $\varphi^{\mathrm{id}} \mathbf{x}_{0} \models$ tll $\mathbf{x}_{0}$. Note that if we change the predicate call from $P_{7}^{\mathrm{id}}$ to $P_{7}^{\rho}$ for a different $\rho$, the parameters in the predicate call have to be reordered in accordance with $\rho$ to obtain a valid entailment $\varphi^{\rho} \models t l l \mathbf{x}_{0}$; hence different permutations induce different equivalence classes. Further details are provided in [30]. As there are six permutations of $\mathbf{x}_{0}$, we conclude

Corollary 3. Let $\Phi$ be the SID defining tll from Example 1. There is a heap automaton $\mathfrak{A}_{\mathrm{t} 11}$ with $8 \cdot 6+1=49$ states that accepts the set $H_{\mathrm{t} 11 \mathbf{x}, \Phi}^{\langle 3\rangle}$.

Remark 6 . One might be tempted to think that we have only moved the complexity into the transition relation and thus gained nothing: Instead of checking entailment w.r.t. tll, we now have to verify entailments w.r.t. several new SIDs. However, the states of our heap automaton coincide with the $\cong_{\text {t11 }}$-equivalence classes. Thus, it always suffices to evaluate the transition relation w.r.t. to small canonical representations of each equivalence class. Hence, instead of solving entailments of the form $P \mathbf{x} \models$ tll $\mathbf{x}$ it suffices to consider entailments $\tau \models P_{i}^{\rho} \mathbf{x}$, where $\tau$ is a reduced symbolic heap. Moreover, since we assume well-determined symbolic heaps, each such $\tau$ has a single tight model up to isomorphism. Then verifying $\tau \models P_{i}^{\rho} \mathbf{x}$ boils down to the model-checking problem for fixed SIDs $P_{i}^{\rho}$. It follows by Remark 3 that $\Delta_{\mathfrak{A}_{\mathrm{t} 11}}$ is decidable in time $\mathcal{O}\left(2^{\text {poly }(\|\Phi\|)}\right)$ (where $\Phi$ is the SID for tll from Example 1). We thus apply Lemma 51 to conclude that Algorithm 2 - if fed with $\mathfrak{A}_{\mathrm{tll}}$ - is an ExPTIME decision procedure for entailments containing tll predicates.

Remark \%. Heap automata allow for easy integration of additional syntactic conditions in order to obtain more compact state spaces. For instance, one observation is that many states of $\mathfrak{A}_{\mathrm{t} 11}$ collapse if reordering of parameters is restricted.

Another approach is to fix the SID in advance and construct an automaton $\mathfrak{A}^{\Phi}=\left(Q, \mathrm{SH}_{\langle\alpha\rangle}^{\Phi}, F, \Delta\right)$ that is only defined on symbolic heaps in $\mathrm{SH}_{\langle\alpha\rangle}^{\Phi}$. Depending on $\Phi$, there may be many $\cong_{P}$-equivalence classes $[\tau] \cong_{P}$ with $[\tau] \cong_{P} \cap \mathrm{SH}_{\langle\alpha\rangle}^{\Phi}=$ $\emptyset$. Dropping such classes can lead to significantly smaller automata. By restricting heap automata in this way, we obtain decision procedures for (established) symbolic heaps with fixed (as opposed to user-defined) SIDs, such as the original decidable symbolic heap fragment introduced by Berdine et al. [3].

\section{A.28.4 Proof of Theorem 13}

Theorem 13 (Myhill-Nerode [34]). Let $P$ be a predicate symbol of an SID $\Phi$. Then there exists a heap automaton $\mathfrak{A}_{P}$ with $L\left(\mathfrak{A}_{P}\right)=H_{P \mathbf{x}_{0}, \Phi}^{\langle\alpha\rangle}$ iff the number of equivalence classes of $\cong_{P}$ is finite. 
Proof. Assume there are only finitely many equivalence classes of $\cong_{P}$. Furthermore, let $[\tau]_{\beth_{P}}$ denote the equivalence class containing formula $\tau$. Then a heap automaton $\mathfrak{A}_{P}=\left(Q, \mathrm{SH}_{\langle\alpha\rangle}, \Delta, F\right)$ accepting $H_{P \mathbf{x}_{0}, \Phi}^{\langle\alpha\rangle}$ is given by $Q=\left\{[\tau]_{\beth_{P}} \mid \tau \in\right.$ $\left.\operatorname{RSH}_{\langle\alpha\rangle}\right\}, F=\left\{[\tau] \cong_{P} \mid \tau \in \mathrm{RSH}_{\langle\alpha\rangle}, \tau \models_{\Phi} P \mathbf{x}_{0}\right\}$ and

$$
\left[\tau_{1}\right] \cong_{P} \ldots\left[\tau_{m}\right] \cong_{P} \stackrel{\varphi}{\rightarrow} \mathfrak{A}_{P}\left[\varphi\left[P_{1} \mathbf{x}_{1} / \tau_{1}, P_{m} \mathbf{x}_{m} / \tau_{m}\right]\right]_{\cong_{P}}
$$

for each symbolic heap $\varphi \in \mathrm{SH}_{\langle\alpha\rangle}$ with $\left\|\Gamma^{\varphi}\right\|=m$. Now, a straightforward induction on the height of unfolding trees reveals for each unfolding tree $t$ and each $q \in Q$, we have

$$
\varepsilon \stackrel{\llbracket t \rrbracket}{\longrightarrow}_{\mathfrak{A}_{P}} q \text { iff } \llbracket t \rrbracket \in q .
$$

Then, by definition of $F$, we have

$$
\tau \models_{\Phi} P \mathbf{x}_{0} \text { iff } \bigvee_{q \in F} \tau \in q \text { iff } \bigvee_{q \in F} \varepsilon \stackrel{\tau}{\rightarrow} \mathfrak{A}_{P} q \text { iff } \tau \in L\left(\mathfrak{A}_{P}\right)
$$

For the converse direction, let $\mathfrak{A}_{P}$ be a heap automaton with $L\left(\mathfrak{A}_{P}\right)=$ $H_{P \mathbf{x}_{0}, \Phi}^{\langle\alpha\rangle}$. Without loss of generality, we assume that $\mathfrak{A}_{P}$ is deterministic, i.e., for each $\tau \in \mathrm{RSH}_{\langle\alpha\rangle}$ there exists exactly one $q \in Q_{\mathfrak{A}_{P}}$ such that $\varepsilon \stackrel{\tau}{\rightarrow}_{\mathfrak{A}_{P}} q$ holds. Otherwise, we may apply the (subset) construction similar to the proof of Lemma 13 to obtain such an automaton (with an exponentially larger state space). Now, for each $q \in Q_{\mathfrak{A}_{P}}$ let

$$
L_{q} \triangleq\left\{\tau \in \operatorname{RSH}_{\langle\alpha\rangle} \mid \varepsilon \stackrel{\tau}{\rightarrow}_{\mathfrak{A}_{P}} q\right\} .
$$

Then, by assumption, we have $L_{p} \cap L_{q}=\emptyset$ for each $p, q \in Q_{\mathfrak{A}_{P}}$ with $p \neq q$. Moreover,

$$
\mathrm{RSH}_{\langle\alpha\rangle} \triangleq \bigcup_{q \in Q} L_{q}
$$

Hence, $\operatorname{Part}\left(\mathfrak{A}_{P}\right) \triangleq\left\{L_{q} \mid q \in Q\right\}$ is a partition of $\mathrm{RSH}_{\langle\alpha\rangle}$. Further, $\tau, \sigma \in$ $L_{q}$ clearly implies $\tau \cong_{P} \sigma$, i.e., our partition $\operatorname{Part}\left(\mathfrak{A}_{P}\right)$ refines the partition induced by $\cong_{P}$. Thus, $\left\|\operatorname{Part}\left(\mathfrak{A}_{P} \|\right) \leq\right\| Q_{\mathfrak{A}_{P}} \|$ is an upper bound on the number of equivalence class of $\cong_{P}$. Since $Q_{\mathfrak{A}_{P}}$ is a finite set, this means that $\cong_{P}$ has only finitely many equivalence classes.

\section{A.29 Details on Heap Automata for Entailment}

\section{Correctness Proof for the Construction in Example 11}

Induced automata. Recall that formulas can only be $P$-equivalent if they have the same number of free variables $\beta$. A heap automaton based on the Myhill-Nerode construction (cf. Theorem 13) for $\mathrm{SH}_{\mathrm{FV} \leq \alpha}$ therefore has states for the equivalence 
classes for each $\beta \leq \alpha$. In Example 11 we, however, restricted ourselves to the classes with exactly $\alpha=3$ free variables. We first justify this choice by noting that the equivalence classes of formulas with exactly $\alpha$ free variables induce all classes for $\beta<\alpha$. This is true in general, not only for the tll case study.

Remark 8. Let $\mathfrak{A}^{=\alpha}$ be a heap automaton obtained by the Myhill-Nerode construction of Theorem 13 restricted to symbolic heaps with exactly $\alpha$ parameters and restricted to acyclic models. Moreover, let each equivalence class be represented by an SID. Then $\mathfrak{A}^{=\alpha}$ induces an automaton $\mathfrak{A}^{=\beta}$ for symbolic heaps with exactly $\beta$ variables for each $\beta<\alpha$.

Proof (Proof sketch). $\mathfrak{A}^{=\beta}$ is obtained by

1. removing all classes that are defined by SIDs that use more than $\beta$ parameters $^{23}$ in points-to assertions, and

2. for the remaining classes, defining new SIDs by adding the closure of the pure formulas, dropping those parts of the pure formulas that do not refer to variables that occur in points-to assertions, and then projecting the remaining parameters onto the first $\beta$ free variables. ${ }^{24}$

Example 12. The automaton derived from Example 11 is the automaton $\mathfrak{A}_{\mathrm{t} 11}^{=3}$ for acyclic symbolic heaps with exactly 3 free variables. The only classes of $\mathfrak{A}_{\mathrm{t} 11}^{=3}$ that do not use all 3 parameters in points-to assertions are the classes $P_{2}^{\rho}, P_{3}^{\rho}$, and $P_{4}^{\rho}$.

For each of these classes, the projection yields the same new classes, defined by the SIDs

$$
\begin{aligned}
& R_{1} \mathbf{x}_{0}[1] \mathbf{x}_{0}[2] \Leftarrow \mathbf{x}_{0}[1] \mapsto\left(\text { null null } \mathbf{x}_{0}[2]\right) \\
& R_{2} \mathbf{x}_{0}[1] \mathbf{x}_{0}[2] \Leftarrow \mathbf{x}_{0}[2] \mapsto\left(\text { null null } \mathbf{x}_{0}[1]\right)
\end{aligned}
$$

Thus, $\mathfrak{A}_{t l l}^{=2}$ has only three states: The two states defined by $R_{1}$ and $R_{2}$ and one state for all other RSHs with two free variables.

The automaton $\mathfrak{A}=1$ has only a single state, because all SIDs in the definition of $\mathfrak{A}_{t l l}^{=3}$ use at least two variables in points-to assertions.

As $\mathfrak{A}_{\mathrm{t} 11}^{=\alpha}$ induces $\mathfrak{A}_{\mathrm{t} 11}^{=\beta}$ for $\beta<\alpha$, it is sufficient to prove the correctness of the automaton $\mathfrak{A}_{\mathrm{tll}}^{=3}$, which we will do in the following.

Overview of the correctness proof. We first recall the definition of tll from Section 1.

$$
\begin{aligned}
\text { tll } & \Leftarrow \mathbf{x}_{0}[1] \mapsto\left(\text { null null } \mathbf{x}_{0}[3]\right):\left\{\mathbf{x}_{0}[1]=\mathbf{x}_{0}[2]\right\} \\
\mathrm{tll} & \Leftarrow \exists \ell r z \cdot \mathbf{x}_{0}[1] \mapsto(\ell r \text { null })
\end{aligned}
$$

$\overline{23}$ Or, more precisely, more than $\beta$ parameters that are pairwise not definitely equal

${ }^{24}$ Note that this is only sufficient when we assume acyclicity. Otherwise, variables that only occur on the right-hand side of points-to assertions can also be identified with variables on the left-hand side, thus also reducing the number of free variables in the symbolic heap. 


$$
* \operatorname{tll} \ell \mathbf{x}_{0}[2] z * \operatorname{tll} r z \mathbf{x}_{0}[3]
$$

Towards a correctness proof for Example 11, we have to show that the predicates defined there (and repeated below) correspond exactly to the $\cong_{\mathrm{t} 11^{-}}$ equivalence classes that contain all reduced symbolic heaps $\tau$ that can be extended to reduced symbolic heaps $\tau^{\prime}$ that entail a tll unfolding, i.e., $\tau^{\prime} \models$ tll x. For brevity, we will henceforth call such symbolic heaps partial models of the tll predicate. ${ }^{25}$ In the following, recall that $\mathbf{y}_{0}[i] \triangleq \rho\left(\mathbf{x}_{0}[i]\right)$.

1. $P_{1}^{\rho} \Leftarrow \mathbf{y}_{0}[1] \mapsto\left(\mathbf{y}_{0}[2] \mathbf{y}_{0}[3]\right.$ null $)$

2. $P_{2}^{\rho} \Leftarrow \mathbf{y}_{0}[1] \mapsto\left(\right.$ null null $\left.\mathbf{y}_{0}[3]\right):\left\{\mathbf{y}_{0}[1]=\mathbf{y}_{0}[2]\right\}$

3. $P_{3}^{\rho} \Leftarrow \mathbf{y}_{0}[1] \mapsto\left(\right.$ null null $\left.\mathbf{y}_{0}[3]\right):\left\{\mathbf{y}_{0}[2]=\mathbf{y}_{0}[3]\right\}$

4. $P_{4}^{\rho} \Leftarrow \mathbf{y}_{0}[1] \mapsto\left(\right.$ null null $\left.\mathbf{y}_{0}[3]\right): \Lambda$ where $\Lambda \triangleq\left\{\mathbf{y}_{0}[1] \neq \mathbf{y}_{0}[2], \mathbf{y}_{0}[2] \neq \mathbf{y}_{0}[3]\right\}$

5. $P_{5}^{\rho} \Leftarrow$ core $\mathbf{y}_{0}$

6. $P_{6}^{\rho} \Leftarrow \exists \ell r z \cdot \mathbf{y}_{0}[1] \mapsto(\ell r$ null $)$

$$
* \operatorname{tll}\left(\ell \mathbf{y}_{0}[2] z\right) * \operatorname{tll}\left(r z \mathbf{y}_{0}[3]\right)
$$

7. $P_{7}^{\rho} \Leftarrow \exists u \ell r z \cdot \mathbf{y}_{0}[2] \mapsto u * \mathbf{y}_{0}[1] \mapsto(\ell r$ null $)$ $* \operatorname{tll}(\ell u z) * \operatorname{core}\left(r z \mathbf{y}_{0}[3]\right)$

8. $P_{8}^{\rho} \Leftarrow \exists u \cdot \mathbf{y}_{0}[2] \mapsto(($ null null $u)) * \operatorname{tll}\left(\mathbf{y}_{0}[1] u \mathbf{y}_{0}[3]\right)$

An illustration of each predicate, together with a graphical representation of a corresponding unfolding, is found in Figure 4.

The correctness proof consists of the following steps.

1. Partition. For each pair of predicates $P_{i}^{\rho}, P_{j}^{\tilde{\rho}}, 1 \leq i, j \leq 8, \rho, \tilde{\rho} \in \mathcal{S}_{\mathbf{x}}$, we show that the sets $\mathbb{U}\left(P_{i}^{\rho} \mathbf{x}\right)$ and $\mathbb{U}\left(P_{j}^{\tilde{\rho}} \mathbf{x}\right)$ are disjoint.

2. Equivalence. We prove for each $\rho$ and each predicate $P_{i}^{\rho}, 1 \leq i \leq 8$, that for all $\tau, \sigma \in \mathbb{U}\left(P_{i}^{\rho} \mathbf{x}\right), \tau \cong_{\mathrm{t} 11} \sigma$ holds.

3. Completeness. We show that the predicates $P_{i}^{\rho}$ define all equivalence classes of partial models, i.e., for each partial model $\tau$ of a tll unfolding there exist an $i$ and a $\rho \in \mathcal{S}_{\mathbf{x}}$ such that $\tau \in P_{i}^{\rho}$.

Once we have established these properties, we immediately obtain a heap automaton for tll-entailment due to Theorem 13.

Partition. We first show that the sets $\mathbb{U}\left(P_{i}^{\rho}\right)$ partition the set of all partial models of tll. To this end, we define for each $1 \leq i \leq 8$ and $\rho \in \mathcal{S}_{\mathbf{x}}$ a set of formulas $\left\{\varphi_{1}^{i, \rho}, \ldots \varphi_{n_{i}}^{i, \rho}\right\}$ with a single predicate call $I$ of arity 3 such that

1. For all $1 \leq k \leq n_{i}$ and for all $\tau \in \mathbb{U}\left(P_{i}^{\rho} \mathbf{x}\right), \varphi_{k}^{i, \rho}[I / \tau] \models \operatorname{tll} \mathbf{x}_{0}$,

\footnotetext{
${ }^{25}$ As we are in a well-determined setting, there is a one-to-one correspondence between reduced symbolic heap and model, so we sometimes use these terms interchangeably. Further, let $\tau \in \mathrm{RSH}$ with $\left\|\mathbf{x}_{0}^{\tau}\right\|=\beta$ and $P \in \operatorname{Pred}(\Phi)$. $\tau$ is a partial model of $P$ if there exists a $\varphi \in \mathrm{SH}^{\Phi}$ with exactly one predicate call $I$ of arity $\beta$, such that $\varphi[I / \tau] \models{ }_{\Phi} P \mathbf{x}_{0}$.
} 
2. For all elements $\sigma \in \mathbb{U}\left(P_{j}^{\tilde{\rho}} \mathbf{x}\right)$ with $\operatorname{ar}\left(P_{j}^{\tilde{\rho}}\right)=\left\|\mathbf{x}_{0}^{\varphi_{k}^{i, \rho}}\right\|$, where $j \neq i$ and/or $\rho \neq \tilde{\rho}$, there exists a $1 \leq k \leq n_{i}$ such that $\varphi_{k}^{i, \rho}[I / \sigma] \not \models \operatorname{tll} \mathbf{x}_{0}$.

In other words, we provide a distinguishing context for each $P_{i}^{\rho}$. Permuting the free variables according to $\rho$ will lead to a permutation of the parameters of the parameter calls in the distinguishing context. To express this in a uniform way, we write $\operatorname{lift}^{\rho}\left(I u_{1} u_{2} u_{3}\right)$ to denote a call to $I$ where the parameters $u_{1} u_{2} u_{3}$ are reordered in accordance with $\rho .{ }^{26}$

- The (single) member of $\mathbb{U}\left(P_{1}^{\rho} \mathbf{x}\right)$ is a binary tree with three nodes ${ }^{27}$ and without linked leaves. The smallest formula yielding a valid tll thus needs to add the two link edges from the left child and the right child and from the right child to its successor.

$\varphi_{1}^{1, \rho} \mathbf{x} \triangleq \exists u \cdot \mathbf{y}_{0}[2] \mapsto($ null null $u) * u \mapsto\left(\right.$ null null $\left.\mathbf{y}_{0}[3]\right) * \operatorname{lift}^{\rho}\left(I \mathbf{y}_{0}[1] \mathbf{y}_{0}[2] u\right)$

- The (single) member of $\mathbb{U}\left(P_{2}^{\rho} \mathbf{x}\right)$ is the smallest valid tll, consisting only of a root (which is thus the leftmost leaf) and its successor. The smallest context yielding a tll is thus the symbolic heap that contains nothing but the predicate call. To distinguish $P_{2}^{\rho}$ from $P_{3}^{\rho}$ and $P_{4}^{\rho}$, we add the pure formula $\mathbf{y}_{0}[1]=\mathbf{y}_{0}[2]$.

$\varphi_{1}^{2, \rho} \mathbf{x} \triangleq \operatorname{lift}^{\rho}(I \mathbf{x}):\left\{\mathbf{y}_{0}[1]=\mathbf{y}_{0}[2]\right\}$

Alternatively, we obtain a tll by using the pointer allocated in $P_{3}^{\rho}$ as the pointer from the leftmost source to an inner leaf.

$\varphi_{2}^{2, \rho} \mathbf{x} \triangleq \exists u v \cdot \mathbf{y}_{0}[1] \mapsto\left(\mathbf{y}_{0}[2] v\right.$ null $) * \operatorname{lift}^{\rho}\left(I \mathbf{y}_{0}[2] u v\right) * v \mapsto\left(\right.$ null null $\left.\mathbf{y}_{0}[3]\right)$

Note that the pure formula in the definition of $P_{2}^{\rho}$ enforces $u=\mathbf{y}_{0}[2]$. The only reason to quantify over $u$ (rather than just writing $\mathbf{y}_{0}[2]$ ) is reusability of the formula in the treatment of $P_{3}$ and $P_{4}$ below

- We get the context for $\mathbb{U}\left(P_{3}^{\rho} \mathbf{x}\right)$ and $\mathbb{U}\left(P_{4}^{\rho} \mathbf{x}\right)$ in the same way as for $\mathbb{U}\left(P_{2}^{\rho} \mathbf{x}\right)$, i.e.,

$\varphi_{1}^{3, \rho} \mathbf{x}=\operatorname{lift}^{\rho}(I \mathbf{x}):\left\{\mathbf{y}_{0}[2]=\mathbf{y}_{0}[3]\right\}$

$\varphi_{1}^{4, \rho} \mathbf{x}=\operatorname{lift}^{\rho}(I \mathbf{x}):\left\{\mathbf{y}_{0}[1] \neq \mathbf{y}_{0}[2], \mathbf{y}_{0}[2] \neq \mathbf{y}_{0}[3]\right\}$

$\varphi_{2}^{3, \rho}=\varphi_{2}^{4, \rho} \triangleq \varphi_{2}^{2, \rho}$

- The partial models generated by $P_{5}^{\rho}$ differ from tlls only in the absent pointer from the rightmost leaf to its successor.

$\varphi_{1}^{5, \rho} \mathbf{x} \triangleq \exists u . \operatorname{lift}^{\rho}\left(I \mathbf{y}_{0}[1] \mathbf{y}_{0}[2] u\right) * u \mapsto\left(\right.$ null null $\left.\mathbf{y}_{0}[3]\right)$

- $P_{6}^{\rho}$ describes all tlls that are obtained by applying the second rule of the tIl-SID at least once. All $\tau \in \mathbb{U}\left(P_{6}^{\rho} \mathbf{x}\right)$ thus already entail a tll unfolding without extension.

$\varphi_{1}^{6, \rho} \mathbf{x} \triangleq \operatorname{lift}^{\rho}(I \mathbf{x})$

- $P_{7}^{\rho}$ describes all tll-like heaps that consist of more than one pointer, lack the successor of the rightmost leaf, but have an additional predecessor of the leftmost leaf. To get a tll, we thus have to both add that last pointer and add a tree-structure that points to the additional leaf at the left as well as the $P_{7}^{\rho}$-root.

$\varphi_{1}^{7, \rho} \mathbf{x} \triangleq \exists u v \cdot \mathbf{y}_{0}[1] \mapsto\left(\mathbf{y}_{0}[2] u\right.$ null $) * \operatorname{lift}^{\rho}\left(I u \mathbf{y}_{0}[2] v\right) * v \mapsto\left(\right.$ null null $\left.\mathbf{y}_{0}[3]\right)$

${ }^{26}$ E.g., if $\rho\left(\mathbf{x}_{0}[i]\right)=3-i$, then $\operatorname{lift}^{\rho}\left(I u_{1} u_{2} u_{3}\right) \triangleq I u_{3} u_{2} u_{1}$.

${ }^{27}$ Because we assume acyclicity, the three nodes are definitely different. 
$-P_{8}^{\rho}$ describes tll-like graphs with an additional predecessor of the leftmost leaf.

$$
\varphi_{1}^{8, \rho} \mathbf{x} \triangleq \exists y \cdot \mathbf{y}_{0}[1] \mapsto\left(\mathbf{y}_{0}[2] \text { y null }\right) * \operatorname{lift}^{\rho}\left(\text { I } y \mathbf{y}_{0}[2] \mathbf{y}_{0}[3]\right)
$$

First observe that for a fixed permutation $\rho$, each pair of classes is separated by at least one formula. Indeed, this separation already occurs for the minimal models of each $P_{i}^{\rho}$ and can thus easily be verified by hand; we omit these tedious constructions. For fixed $\rho$, this shows the pairwise disjointness of the $P_{i}^{\rho}$ classes.

Now let $\sigma \in \mathbb{U}\left(P_{i}^{\rho} \mathbf{x}\right), \zeta \in \mathbb{U}\left(P_{i}^{\tilde{\rho}} \mathbf{x}\right)$, for $\rho \neq \tilde{\rho} \in \mathcal{S}_{\mathbf{x}}$. We have $\varphi_{1}^{i, \rho}[I / \sigma] \models$ $\operatorname{tll} \rho\left(\mathbf{x}_{0}\right)$, and $\varphi_{1}^{i, \tilde{\rho}}[I / \zeta] \models \operatorname{tll} \tilde{\rho}\left(\mathbf{x}_{0}\right)$, but $\mathbb{U}(\operatorname{tll} \rho(\mathbf{x})) \cap \mathbb{U}(\operatorname{tll} \tilde{\rho}(\mathbf{x}))=\emptyset$. Thus we also have that, for fixed $i$ and $\rho \neq \tilde{\rho} \in \mathcal{S}_{\mathbf{x}}, \mathbb{U}\left(P_{i}^{\rho} \mathbf{x}\right) \cap \mathbb{U}\left(P_{i}^{\tilde{\rho}} \mathbf{x}\right)=\emptyset$ :

Putting this together with the arguments above, we obtain a proof for each $i, j \in\{1, \ldots, 8\}, \rho, \tilde{\rho} \in \mathcal{S}_{\mathbf{x}}$, that if $i \neq j$ or $\rho \neq \tilde{\rho}$, then $\mathbb{U}\left(P_{i}^{\rho} \mathbf{x}\right) \cap \mathbb{U}\left(P_{j}^{\tilde{\rho}} \mathbf{x}\right)=\emptyset$.

$\cong_{\mathrm{t} 11}$-Equivalence. For $1 \leq i \leq 4$, the set $\mathbb{U}\left(P_{i}^{\rho}\right)$ is a singleton, i.e. there is nothing to show. Thus, let $i>4$. Formally, the remaining cases are proven individually by coinduction: We assume for each $1 \leq i \leq 8$ that all reduced symbolic heaps in $\mathbb{U}\left(P_{i}^{\rho}\right)$ are already known to be equivalent and then, for each rule with left-hand side $P_{i}^{\rho}$, show that the unfolding obtained from replacing each predicate call by one of these reduced symbolic heaps is again equivalent.

We omit these tedious calculations and only provide a rough intuition for each case. Let $\tau \in \mathbb{U}\left(P_{i}^{\rho}\right)$ and $\varphi$ be a symbolic heap with one predicate call $I \mathbf{x}$ such that $\varphi[I / \tau] \models_{\Phi}$ tll $\mathbf{x}_{0}$. Such a symbolic heap always exists, because each unfolding can be extended to a TLL. We proceed by case distinction to see that $\varphi$ and $\tau$ are always of the same form - except for the size of the respective TLL.

- For $i=5$, it is easy to verify that exactly one pointer - the one to the rightmost leaf - is missing in $\tau$. Thus, $\varphi$ is always a TLL with an additional pointer to the rightmost leaf in which the rightmost subtree, $\tau$, is missing (except for the additional pointer), e.g. $\varphi_{5}^{i, \rho}$.

- For $i=6, \tau$ is a non-empty TLL. Thus, $\varphi$ always corresponds to one of the tll rules with the single predicate being tllx , e.g. $\varphi_{6}^{i, \rho}$.

- For $i=7, \tau$ is a TLL that lacks the pointer to the rightmost leaf and has an additional pointer to the leftmost leaf. Thus, $\varphi$ always corresponds to a tree-structure lacking a pointer to the rightmost leaf and an additional isolated pointer, e.g. $\varphi_{7}^{i, \rho}$.

- For $i=8, \tau$ is a TLL with an additional incoming pointer at the leftmost leaf. Thus, $\varphi$ always corresponds to a TLL lacking the rightmost subtree, e.g. $\varphi_{8}^{i, \rho}$.

Completeness. We now show that the $8 \cdot 6$ predicates $P_{1}^{\rho} \ldots P_{8}^{\rho}, \rho \in \mathcal{S}_{\mathbf{x}}$, cover all partial models of tll unfoldings.

The basic idea is to show that every partial model of tll is either in one of the sets $\mathbb{U}\left(P_{i}^{\rho} \mathbf{x}\right)$ or has at least four free variables. ${ }^{28}$ To this end, we first develop a sufficient condition for concluding that a partial model needs at least four free

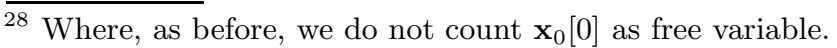


variables. Recall that we assume reduced symbolic heaps under consideration to be well-determined. Since there exists exactly one tight model, i.e. a stack-heap pair, up to isomorphism for each such symbolic heap, we will argue about the structure of such a stack-heap pair instead.

Intuitively, a location that occurs in two separated heaps, i.e. $h \uplus h^{\prime}$, is called a shared location. Formally, for a heap $h$, we write $\operatorname{Loc}(h)$ to denote all locations allocated or referenced - pointed to - to in $h$, i.e. $\operatorname{Loc}(h)=\operatorname{dom}(h) \cup \operatorname{codom}(h)$.

Definition 26 (Shared Location). Let $(s, h) \in$ States be a stack-heap pair and $h^{\prime}$ be a heap such that $h \uplus h^{\prime}$ is well-defined. Then, a location $\ell \in \operatorname{Loc}(h)$ is $a$ shared location of $h$ w.r.t. $h^{\prime}$ if $\ell \in \operatorname{Loc}\left(h^{\prime}\right)$ or $\ell \in \operatorname{codom}(s)$.

Lemma 52. Let $\left(s, h_{1} \uplus h_{2}\right)$ be the unique tight model up to isomorphism of $\varphi[I / \tau]$ for some partial model $\tau$ of $P$ such that $\left(s, h_{1}\right)$ is a model of $\tau$. If $h_{1}$ has $\alpha$ shared locations w.r.t. $h_{2}$ then $\tau$ has at least $\alpha$ free variables.

Proof (Proof sketch). Since $\tau$ is established, equalities and reachability between symbolic heaps and unfoldings of their predicate calls have to be propagated through predicate calls (cf. Lemma 28 and Lemma 34). Thus, if $h_{1}$ has $\alpha$ shared locations w.r.t. $h_{2}, \tau$ has at least $\alpha$ free variables.

To show the completeness of the equivalence classes $\mathbb{U}\left(P_{i}^{\rho} \mathbf{x}\right)$, it thus suffices to show that every well-determined partial model is either in one of the classes or its unique tight model has at least four shared locations.

Lemma 53. Every $\tau \in \mathrm{RSH} \backslash \bigcup_{1 \leq i \leq 8, \rho \in \mathcal{S}_{\mathbf{x}}} P_{i}^{\rho}$ that is a partial model of tll has a unique tight model with at least four shared locations.

Proof. Note that tll-unfoldings contain only two types of points-to assertions: $x \mapsto(y z$ null) and $x \mapsto($ null null $y$ ) (for some variables $x, y, z)$. Let $(s, h)$ be the unique tight model of some partial model $\tau$ of tll. Due to the one-to-one correspondence between $\tau$ and $(s, h)$, we will not distinguish between $(s, h)$ and $\tau$ in the remainder of the proof.

We refer to heap entries corresponding to the first kind of points-to assertion, i.e. $h(u)=\left({ }_{-}\right.$, , null), as $i$-pointers (short for inner pointers). Moreover, we refer to heap entries corresponding to the second kind of points-to assertion, i.e. $h(u)=($ null, null, _) , as $\ell$-pointers (short for leaf pointers). In both cases, given $h(u)=\left(v_{1}, v_{2}, v_{3}\right)$, we refer to $u$ as $i$-source and $\ell$-source and to $v_{1}, v_{2}, v_{3}$ as $i$-target and $\ell$-target, respectively.

Recall that we restricted our attention to those calls of tll $\mathbf{x}$ whose parameters are non-null and pairwise definitely unequal, where the latter point is a consequence of the assumption of acyclicity. ${ }^{29}$

We make several observations regarding the shared locations of partial tll models $\tau$ based on the properties of tll unfoldings.

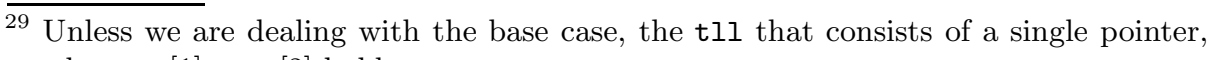
where $\mathbf{x}_{0}[1]=\mathbf{x}_{0}[2]$ holds. 
1. In a tll, there is at most one $i$-source that is no $i$-target (the root). Thus, if $\tau$ contains $n$ distinct $i$-sources that are not $i$-targets, this requires $n-1$ distinct shared locations.

2. In a tll, every $i$-target that is not an $i$-source is an $\ell$-source. Thus, if $\tau$ contains $n$ distinct $i$-targets that are neither $i$-sources nor $\ell$-sources, this requires $n$ shared locations.

3 . In a $\mathrm{tll}$, if there is at least one $i$-location, then all $\ell$-locations are $i$-targets. Thus, in such $\tau$, all $\ell$-sources that are not $i$-targets are shared locations.

4. In a tll, there is only one $\ell$-source that is no $\ell$-target (the leftmost leaf), and there is only one $\ell$-target that is no $\ell$-location (the successor of the rightmost leaf) Thus, if $\tau$ contains $n$ distinct $\ell$-sources that are not $\ell$-targets, this requires $n-1$ distinct shared locations. Likewise, if $\tau$ contains $n$ distinct $\ell$-targets that are not $\ell$-sources, this requires $n-1$ distinct shared locations. (Note that we have to take care not to count these shared locations twice, because of possible overlap with the previous point.)

5 . In a tll, all roots of the trees induced by considering only $i$-sources must be referenced by free variables (the locations in the corresponding model belong to $\operatorname{codom}(s))$. Thus, $\tau$ has one shared location per such $i$-tree.

6. In a $t l l$, in a sequence of linked $\ell$-sources, both the first $\ell$-source and the last $\ell$-target must be referenced by free variables.

Thus, the leftmost $\ell$-source and the successor of the rightmost $\ell$-source are shared locations for every maximal linked list of $\ell$-sources in $\tau$.

Based on these observations, we make a case distinction over the possible structure the unique tight model of a partial model $\tau$ interpreted as a graph and show that each graph either corresponds to a $\tau \in \mathbb{U}\left(P_{i}^{\rho} \mathbf{x}\right)$ for some $i, \rho$ or corresponds only to $\tau$ with at least four shared locations .

$-\tau$ contains only $\ell$-sources.

- If it contains only a single source, then $\tau \in \mathbb{U}\left(P_{i}\right)$ for $i \in\{2,3,4\}$.

- If it consists of two sources, then $\tau \in \mathbb{U}\left(P_{7}\right)$.

- If it contains $n \geq 3$ sources, then $\tau$ has $n$ shared locations plus the one for the successor of the last leaf, i.e, at least four.

$-\tau$ contains only $i$-sources

- If it contains only a single source, $\tau \in \mathbb{U}\left(P_{1}\right)$

- If it contains at least two sources, then it contains at least three $i$-targets that are neither $i$-sources nor $\ell$-sources; in addition, it contains at least one root, which also has to be a shared location; yielding a total of at least four shared locations

- If it contains both $i$ - and $\ell$-sources, it needs one shared location per root and two shared locations per maximal list of linked leaves. ${ }^{30}$

- If it contains two roots, it thus has at least four shared locations

- If it contains two unconnected lists of linked $\ell$-sources, it has at least five shared locations

30 This is only true because we assumed the last node in a TLL must not be equal to null nor create a cycle. 
- If it contains only one list of linked leaves, but one of the inner nodes of these leaves is no $i$-target, it has at least four shared locations

- If it has at most three shared locations, it thus has exactly one root, and at most its first and its last $\ell$-source are not $i$-targets (but each of them can be). Thus, $\tau \in \mathbb{U}\left(P_{i}\right)$ for some $5 \leq i \leq 8$.

Corollary 4. The set $\bigcup_{1 \leq \leq 8, \rho \in \mathcal{S}_{\mathbf{x}}} \mathbb{U}\left(P_{i}^{\rho}\right)$ contains all well-determined partial tıl models that can be expressed with at most three free variables.

Corollary 5. The set $\left\{P_{i}^{\rho} \mid 1 \leq i \leq 8, \rho \in \mathcal{S}_{\mathbf{x}}, \Lambda \in \operatorname{Pure}\left(\mathbf{x}_{0}\right)\right\}$ of equivalence classes of well-determined partial tนl models is complete.

\section{Heap Automata for Singly-linked List Segments}

Recall the SID for acyclic singly-linked list segments from Example 1. A heap automaton $\mathfrak{A}$ for $\mathbf{s l l}$ is defined in Fig. 5 .

$Q \triangleq\left\{q_{\mathrm{eq}}, q_{\mathrm{diff}}, q_{\mathrm{rev}}, q_{\mathrm{fst}}, q_{\mathrm{snd}}, q_{\perp}\right\} \quad F \triangleq\left\{q_{\mathrm{eq}}, q_{\mathrm{diff}}, q_{\mathrm{fst}}\right\}$

Transitions:

$$
\begin{array}{ll}
\left(\varepsilon, \tau, q_{\mathrm{eq}}\right) \in \Delta & \text { iff } \left.\tau \models \operatorname{emp}:\left\{\mathbf{x}_{0}[1]=\mathbf{x}_{0}[2]\right)\right\} \\
\left(\varepsilon, \tau, q_{\text {diff }}\right) \in \Delta & \text { or } \tau \models \operatorname{emp} \wedge\left\|\mathbf{x}_{0}^{\varphi}\right\|=2 \\
\left(\varepsilon, \tau, q_{\text {rev }}\right) \in \Delta & \text { iff } \tau \models \operatorname{sll} \mathbf{x}_{0}[1] \mathbf{x}_{0}[2]:\left\{\mathbf{x}_{0}[2] \neq \mathbf{n u l l}\right\} \\
\left(\varepsilon, \tau, q_{\text {fst }}\right) \in \Delta & \text { iff } \tau \models \operatorname{sll} \mathbf{x}_{0}[2] \mathbf{x}_{0}[1]:\left\{\mathbf{x}_{0}[1] \neq \mathbf{n u l l}\right\} \\
\left(\varepsilon, \tau, q_{\text {snd }}\right) \in \Delta & \text { iff } \tau \models \operatorname{sll} \mathbf{x}_{0}[1] \mathbf{n u l l} \\
\left(\varepsilon, \tau, q_{\perp}\right) \in \Delta & \text { iff } \tau \models \operatorname{sll} \mathbf{x}_{0}[2] \text { null } \\
(\mathbf{q}, \varphi, q) \in \Delta & \text { iff } \tau \not \models \operatorname{sll} \mathbf{x}_{0} \\
& \text { iff }\left(\varepsilon, \varphi\left[P_{1} / \rho_{\mathbf{q}[1]}, \ldots, P_{m} / \rho_{\mathbf{q}[m]}\right], q\right) \in \Delta
\end{array}
$$

\section{Representations:}

$$
\begin{aligned}
\rho_{q_{\mathrm{eq}}} \triangleq \mathrm{emp}:\left\{\mathbf{x}_{0}[1]=\mathbf{x}_{0}[2]\right\} \\
\rho_{q_{\mathrm{diff}}} \triangleq \mathbf{x}_{0}[1] \mapsto\left(\mathbf{x}_{0}[2]\right):\left\{\mathbf{x}_{0}[2] \neq \text { null, } \mathbf{x}_{0}[2] \neq \mathbf{x}_{0}[1]\right\} \\
\rho_{q_{\mathrm{rev}}} \triangleq \mathbf{x}_{0}[2] \mapsto\left(\mathbf{x}_{0}[1]\right):\left\{\mathbf{x}_{0}[1] \neq \text { null, } \mathbf{x}_{0}[2] \neq \mathbf{x}_{0}[1]\right\} \\
\rho_{q_{\mathrm{fst}}} \triangleq \mathbf{x}_{0}[1] \mapsto(\text { null }) \\
\rho_{q_{\mathrm{snd}}} \triangleq \mathbf{x}_{0}[2] \mapsto(\text { null }) \\
\rho_{q_{\perp}} \triangleq \mathbf{x}_{0}[1] \nRightarrow \mathbf{x}_{0}[1]
\end{aligned}
$$

Fig. 5. A heap automaton $\mathfrak{A}=(Q,\langle\alpha\rangle, \Delta, F)$ with $L(\mathfrak{A})=H_{\mathrm{s} 11 \mathrm{x}, \Phi}^{\langle\alpha\rangle}$, for acyclic singlylinked list fragments sll as defined in Ex. 1; plus canonical representations $\rho_{q}$ for each state $q$.

Observe that $\Delta$ is compositional. Note further that we have defined $\Delta$ in such way that $(\varepsilon, \tau, q) \in \Delta$ for $q \in F$ iff $\tau \models \operatorname{sll} \mathbf{x}_{0}$, i.e., $L(\mathfrak{A})=H_{\operatorname{sll} \mathbf{x}, \Phi}^{\langle\alpha\rangle}$. 
Figure 5 also shows the canonical representations of each state, i.e., the minimal unfoldings of each state's formula. These are the symbolic heaps that are substituted into the predicate calls in symbolic heaps $\varphi$ to obtain simple entailment problems for deciding transitions $(\mathbf{q}, \varphi, q)$.

\section{Optimizations when the Left-Hand Sides of Entailments are Restricted}

Restrictions on parameter reordering. First of all, recall why the automaton from Corollary 3 had $8 \cdot 6+1$ states:

- For a fixed parameter ordering, there are 8 equivalence classes of partial models

- For each class, we have to differentiate between all $3 \cdot 2 \cdot 1=6$ permutations of the three free variables $\mathbf{x}_{0}[1], \mathbf{x}_{0}[2], \mathbf{x}_{0}[3]$ : While the permutation of free variables does not change that a symbolic heap can be extended to entail a tll unfolding, it changes how it can be extended, because the extension may need to reorder parameters based on the permutation.

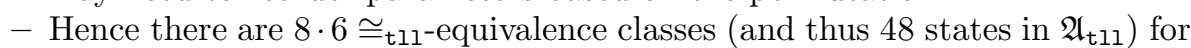
partial models.

- There is one $\cong_{\text {t1l }}$ equivalence class for symbolic heaps that cannot be extended to entail a tll unfolding, which corresponds to a sink state.

It will, however, often not be necessary to deal with all such permutations in practice. For example, just by requiring that all predicates used in the program that define tree-like structures use their first parameter to identify the root, merely two possible permutations remain. We can thus specify a tll automaton with just $8 \cdot 2+1=17$ states that guarantees a sound analysis as long as the predicates used in the program satisfy the aforementioned assumption.

Fixing SIDs in advance. In practice, the fact that heap automata are independent of specific SIDs may also lead to a needless blow-up of the state space. On the one hand, this independence is a useful feature that enables reusability of automata across analyses. On the other hand, every program analysis on a fixed program will usually be performed w.r.t. a fixed SID $\Phi$. It may thus make sense to perform SID-specific reductions of heap automata to boost the performance of the analysis on specific programs of interest.

Such optimizations are based on the observation that the set of reduced symbolic heaps that can be derived from a fixed SRD $\Phi$ will often not intersect every single $\cong_{P}$ equivalence class. Formally, given an equivalence class $[\tau] \cong_{P}$, where we write $[\tau] \cong_{P}$ to denote the equivalence class that contains $\tau$ (cf. Theorem 13), it may turn out that $[\tau] \cong_{P} \cap \mathrm{SH}_{\mathcal{C}}^{[\Phi]}=\emptyset$.

If we know that all formulas that occur on the left-hand side of entailments only contain predicates from $\Phi$, we can drop all the states that correspond to equivalence classes $[\tau] \cong_{P}$ with $[\tau] \cong_{P} \cap \mathrm{SH}_{\mathcal{C}}^{[\Phi]}=\emptyset$. A heap automaton will therefore often be much smaller if we build it for entailment w.r.t. a fixed SID $\Phi$ rather than for arbitrary formulas in $\mathrm{SH}_{\langle\alpha\rangle}$. 
This becomes particularly clear if we want to reimplement decision procedures for fixed SIDs such as the one by Berdine et al. [3] within our framework. If, for example, formulas on the left-hand side of the entailment may only refer to the sll predicate (as is the case in the work by Berdine et al. [3]), we can simplify the automaton $\mathfrak{A}$ from Figure 5 as follows. We can drop the states $q_{\text {rev }}$ and $q_{\text {snd }}$, because we know in advance that there is no predicate in our SID that defines singly-linked lists in reverse order. Even in this simple case, we can thus reduce the size of the state space by a third. In more complex cases, even larger reductions are possible. 